\title{
ASPECTS, The Mismeasure of Stroke: A Metrological Investigation
}

\author{
Richard A. Suss, M.D. \\ Richard.Suss@UTSouthwestern.edu
}

Neuroradiology Section, Department of Radiology

The University of Texas Southwestern Medical Center

5323 Harry Hines Boulevard, Dallas, TX 75390-9178

OSF Preprints: https://doi.org/10.31219/osf.io/c4tkp or https://osf.io/c4tkp/

Versions: 2019: 1 (Dec. 1), 2, 3 (Dec. 29, 31); 2020: 4 (May 15), 5 (Dec. 31); 2021: 6 (Dec. 12).

Abstract -ASPECTS (Alberta Stroke Program Early CT Score) is an irregular scoring system that reads out as 10 to 0 points. It was originally conceived to reflect acute MCA (middle cerebral artery) territory infarct volume on (plain) CT, but it is equally applicable to and testable by MRI.

Background material prepares the reader by explaining pertinent measurement principles and ASPECTS's genesis, definitions, claims, and quirks. This report investigates ASPECTS as a volume surrogate without independently advocating for or against the therapies it might help plan.

Method: The original authors of four publications provided their unpublished primary numerical data for further analysis. A CT study expands on the ACCESS database, which compares ASPECTS with two other subjective infarct scales: the legacy 1/3 MCA estimate and the multivariate IST-3 scale. An MRI study pools three diffusion-weighted (DWI) series as a patient-level meta-analysis unifying their comparisons of ASPECTS to the volumes that were found by semiautomated measurement.

CT results: ASPECTS is unreliable, showing wide interrater variation with three quantifiable effects. It delivers little more than half as much entropy reduction as the IST-3 scale shows CT can support. Its large standard deviation $(S D)$ takes up much of the scale width. Converting $S D$ to a loss function with respect to a reference standard neuroradiologist gives an alarming weighted measure of error in ratings.

MRI results: There are many-fold ranges of volume per ASPECTS and of ASPECTS per volume, causing sometimes large misclassifications one or both ways by any ASPECTS dichotomization. Looking past the ranges to averages, an estimate of $1 / 3$ of the MCA territory corresponds best to ASPECTS cutting between 6 and $5(6 / / 5)$ and a volume of $65 \mathrm{~cm}^{3}(<1 / 4$ of the average MCA territory).

Discussion: There is already a simple, quick, and reliable manual volume measure $(A B C / 2,2 S h / 3)$. An attempt to salvage ASPECTS by reinterpreting its purpose does not hold up under scrutiny. ASPECTS can be replaced in stroke guidelines: the guideline cutting at ASPECTS 6//5 is consistent on average with the increasingly commonly stated, and better defined, $70 \mathrm{~cm}^{3}$ threshold. Arguments defending ASPECTS are rebutted by the evidence herein and by literature citations.

Conclusion: ASPECTS subtracts value from the more objective direct volume measurements that are universally available by manual calculation and are becoming available by automatic software. ASPECTS inherently risks clinically significant misclassification (harm) for many patients. 
Page 2 ASPECTS, The Mismeasure of Stroke: A Metrological Investigation

\section{Acknowledgments}

My local colleague and friend, Marco Pinho, recruited me for this project. Thank you, Marco! Ke Lin, Constance de Margerie-Mellon, Julian Schröder, J. M. Wardlaw and Francesca Chappell shared data from their studies. J. M. Wardlaw sent an otherwise unavailable article and alerted me to other articles, all carefully read. Ke Lin, J.M. Wardlaw, Tudor Jovin, Keith Muir, Greg Albers, Francesca Chappell, and Beverly Houghton corresponded with me on various topics. Marco Pinho, Ke Lin, and J.M. Wardlaw commented on full drafts, each sharing significant insights or advice. Several members of my Neuroradiology Section and Radiology Department, whose residents' misgivings about ASPECTS amplified my original interest, expressed support for this investigation. In particular, Merissa Harris, Will Moore, Michael Achilleos, John Barr, Amit Agarwal, Joseph Maldjian, and Devan Moody made helpful remarks or offered valuable suggestions, Kelly Tornow based a neuroradiology journal club presentation on two of the references, Lee Pride provided \$7's critical case in point and contributed to its description and Lauren Kolski and Jacob Fleming assisted in its preparation. My wife, Shelley, graciously shared me with this project and critiqued parts of several drafts. I am indebted to all of these individuals for their generosity, noting that their mention does not imply endorsement of this work by them.

Through it all, I retain sole responsibility for the compilation and for its errors of commission and omission, and would appreciate feedback for possible future versions.

Prospective readers - from students, residents and fellows, to radiologists, neurologists and other clinicians, and to research scientists and administrators - are kept in mind for their time, interest, and labor, in the arrangement of the material. Depending on the desired topics and depth, anyone might read just the Synopsis, or selected sections such as the central data section (§6), or the full main text alone, or perhaps also explore the footnotes which supplement the arguments and list page numbers for the convenience of verifying or exploring the references.

\section{Version Changes}

Version 2 makes clarifications and additions on pages 2, 19-21, 24, 27-28, 45, 48-49, 60-63, 70, 74, and 76-78, and adds 20 new references (now 537 and with digital object identifiers if available), to reinforce the point that the use of ASPECTS is a suboptimal and superfluous practice.

Version 3 adds to Page 1 this article's OSF (Open Science Framework) Preprints digital object identifier (doi).

Version 4 corrects minor technical errors; tweaks wording, typography, and graphics; credits Cavalieri's principle; augments page 67's last paragraph; discriminates the suitabilities of "satisficing"; notes a 207-word summary at https://doi.org/10.3174/ajnr.A6485; and adds references: one for "data theory" on page 7, three for "QIB" on page 49, two more for " $A B C / 2$ " on page 52, as well as Yoo et al. 2012, Goyal et al. 2016b, Sheth et al. 2019, and Qiu et al. 2020. Text pagination and most footnote numbers are unchanged.

Version 5 cites, on page 49, a head-to-head comparison of auto-ASPECTS and auto-volume applying the same software package to baseline CTs (Brugnara et al. 2020). Footnote numbers and pagination are otherwise unchanged.

Version 6 is a miscellaneous update and bug-fix, including repair of some dead links in the bibliography. The history of "ABC/2" on page 51 has additions and corrections. "Best Fit Method" is quoted help explain $\S 7$. Footnote numbers and pagination remain unchanged. 


\section{ASPECTS, The Mismeasure of Stroke: A Metrological Investigation}

\section{Contents}

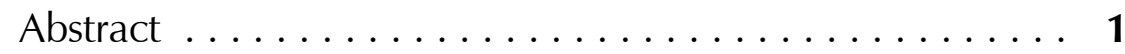

Acknowledgments ................... 2

Version Changes ..................... 2

Introductory Material and Essential Background

\$1. Preface ....................... 4

Synopsis ................... 5

\$2. Ground Rules of Measurement . . . . . . . . . . . . 7

$\$ 2.1$ Scales ...................... 7

$\$ 2.2$ Purpose ...................... 8

$\$ 2.3$ Precision and Accuracy.................. 9

$\$ 2.4$ Getting It Wrong-The Loss Function . . . . . . . . 10

$\$ 2.5$ Correlation and Surrogacy . . . . . . . . . . . . . . 12

\$3. The Development of ASPECTS . . . . . . . . . . . . . 13

$\$ 3.1$ ASPECTS Invented. . . . . . . . . . . . . . . . 13

$\$ 3.2$ ASPECTS Taught ................. 16

\$4. The MCA Territory and Its " $1 / 3$ " Rule . . . . . . . . . 22

\$5. Kappa Agreement . . . . . . . . . . . . . . . . . . . . . 29

Reanalysis of Published Data

§6. How Not to Measure Volume: ASPECTS . . . . . . . . . . 34

$\$ 6.1$ The Real Anatomy of ASPECTS . . . . . . . . . . 34

$\$ 6.2$ A Further Analysis of Some CT-ASPECTS Data . . . 38

$\$ 6.3$ A Patient-Level Pooling of DWI-ASPECTS Data .... 45

\section{Discussion Topics}

\$7. How To Measure Volume: $A B C / 2,2 S h / 3 \ldots \ldots \ldots \ldots \ldots 51$

\$8. ASPECTS Repurposed . . . . . . . . . . . . . 60

\$9. Whither ASPECTS? . . . . . . . . . . . . . . . . 64

\section{Summation and Conclusion}

\$10. Looking Back At and Forward From ASPECTS . . . . . . . . 69

References ........................ 79

Special Symbols and Abbreviations ............. 99 
Page 4 ASPECTS, The Mismeasure of Stroke: A Metrological Investigation

\section{§1. Preface}

ASPECTS is the Alberta Stroke Program Early CT Score, a surrogate for infarct volume (\$3, §6). $\mathrm{CT}$ is $\mathrm{x}$-ray computed tomography, but the Score applies also to magnetic resonance imaging (MRI). In this report, $\$ 6.2$ reanalyzes data from a CT series comparing 3 scales using 32 scans and 259 raters, and §6.3 assembles a patient-level meta-analysis of 3 MRI series (949 patients: ASPECTS vs. volume). They both show ASPECTS to be an erratic measure. Background sections address foundational issues in measurement $(\mathbf{\$ 2})$ and ASPECTS's motive and method $(\$ 3)$, and review its primary claims $(\$ 4, \S 5)$. The innovative $\$ \mathbf{6}$ assesses ASPECTS as a measurement for its target, infarct volume. \$7 describes an efficient alternative. $\$ 8$ rebuts a revisionist rebuttal. $\$ 9$ looks at guidelines using ASPECTS. $\$ 10$ sums up pros and cons and looks ahead. Flanking §6, §§1-5 collect essential introductory context, and \$§7-10 elaborate on §6's methodological implications and potential clinical significance.

Accordingly, while the broad setting is early acute brain infarct therapy by human recombinant tissue plasminogen activator (tPA, alteplase) or embolectomy, this report is a logic- and data-driven examination of measure choice and measurement technique taking no position on the therapies themselves. Infarct volume must be respected as a quantity. The use of ASPECTS as its surrogate is criticized in the light of definitions, measurement skills, testing, and common sense.

"Metrology is the science of measurement." Without becoming professional metrologists (minders of the measures), we still depend on concepts and tools that refer back to their discipline.

I often say that when you can measure what you are speaking about, and express it in numbers, you know something about it; but when you cannot measure it, when you cannot express it in numbers, your knowledge is of a meagre and unsatisfactory kind; it may be the beginning of knowledge, but you have scarcely, in your thoughts, advanced to the stage of science.... ${ }^{3}$

This opinion by William Thomson (since 1892, Lord Kelvin), that expression-by-numbers is necessary for science, might carelessly be converted to a feeling that it suffices. The feeling might be reinforced by statistical correlation, statistical significance, and claims of statistical validation, without policing the sources. But science has more necessary conditions than just numbers.

Thomson calculated that the sun had illuminated the earth for $<100,000,000$ years. This might be forgiven, ${ }^{4}$ but his prestige effectively "bludgeoned" some contemporary geologists into conformity ${ }^{5}$ grounds to criticize Thomson and his followers. There are more immediate grounds to criticize the ASPECTS literature and its own bandwagon effect. §6's numerical data, and some of the literature itself as another form of data, show that ASPECTS yields numbers heedless of sound metrology, acting as a distorted and often misleading ruler for infarct extent and therapy choice.

\footnotetext{
1 Zivin et al. 1985.

2 Sené et al. 2017, p. 397 (ital. added); p. 398: "Researchers often treat such [measurement] tools as a 'black box'... on trust ...."

3 Thomson 1883, p. 73.

4 Thomson 1862, p. 393. Atomic structure and nuclear energy were unknown.

5 Carey 1988, pp. 71-5.
} 


\section{Synopsis}

This preview of the facts and analysis set forth in $\$ \S 2-10$ is shorn of their authenticating details, quotations, and citations. It serves instead to help the reader gain an early overview of the structured presentation to follow.

Because this report is fundamentally about measurement, $\$ 2$ clarifies the pertinent ground rules. That qualitatively different kinds of measurement scales exist may be intuitive, but it probably benefits from explicit review before they come into play in assessing ASPECTS $(\$ \S 3, \ldots)$. Then a measurement's purpose must be kept in mind both to choose a scale and to prevent the task from degenerating into a pro forma numerical exercise. The measurement's purpose indicates the needed precision and accuracy, with risks from error that may scale nonlinearly and differently in the opposed directions. When a target quantity is inaccessible to direct measurement, attention typically shifts to a supposed correlate. Medicine has myriad numerical correlations with various degrees of weakness. Surrogate variables, and surrogates for surrogates, can propagate medical errors, uncertainties, and controversies. ASPECTS is a superfluous surrogate for a surrogate.

As intravenous thrombolysis (clot dissolution) for brain infarcts came under study in the 1980-90s, fear of its main side effect, symptomatic brain hemorrhage, could inhibit its use for large infarcts. A plausible arbitrary upper limit equivalent to one-third of the middle cerebral artery (MCA) territory was erected and named the $1 / 3$ MCA rule $(\mathbf{\$ 3 . 1}, \S 4)$. Inconveniently,(1) the MCA territory volume varies, (2) its boundaries and volume are not demarcated and are therefore unknown in a given individual, (3) no default average volume was assigned for reference, although perhaps one was being guessed, (4) the task was cognitively convoluted, to integrate a volume fraction from planar CT images, and (5) the original field of interest for infarcts was cerebral "hemispheric," not just the "MCA" territory. No technique was described to estimate " $1 / 3$," nor did rigorously quantitative verification follow. Overlooking the feasibility of settling (3) and of using a geometric approximation for (4), a group in Calgary, Alberta, tried to bypass (1)-(5) with a scoring system, ASPECTS (\$3.1). The rationale was to address the supposed difficulty of estimating a tissue volume, $1 / 3$ of the (average?) MCA territorynot to replace the measure, volume. This investigation carefully describes its subject, ASPECTS. The Calgary group assigned equal points to 10 regions of very unequal sizes, while failing to justify or even acknowledge the anomaly of this unitization. Moreover, the six raters in the group's how-to article interpreted ASPECTS's vague definitions diversely (\$3.2). Some inconsistencies persist in manual and software practice up to the present, despite later reviews attempting to update and clarify ASPECTS.

ASPECTS was claimed to correlate with outcome measures such as hemorrhage and disability (\$4) and to outperform the $1 / 3$ rule on the process measure of interrater agreement (\$5). But correlation is fickle, attending poorly to individual patients, and for ASPECTS it was seldom compared to a true volume measurement technique. Reliability merely as agreement is no virtue without accuracy. Early claims of sharp discrimination and superior interrater agreement did not pan out. 
Page 6 ASPECTS, The Mismeasure of Stroke: A Metrological Investigation

ASPECTS is badly out of step with its original target of infarct volume (\$6). As volume is a $3 / 2$ power of area, the deep ASPECTS regions (insula, caudate and lentiform nuclei, and internal capsule posterior limb) are proportionally even smaller than suggested by their sectional areas, dwarfed by each of the six cortical-subcortical regions (M1-M6). The four deep regions, $40 \%$ of ASPECTS's points, total only $\approx 8 \%$ of the MCA territory's volume. This imbalance is not ASPECTS's only inherent bias. Because a point is subtracted (from 10) integrally only, a threshold must be set for counting a region as being involved. The original and still official threshold is any perceptible involvement. Depending on how an infarct (or spotty collection of them) falls in relation to the region borders, even a small volume can claim a lot of points and can wrongly promote a patient to an infarct category considered futile for thrombectomy. Some writers admit to fudging ASPECTS by ignoring small involvements, less than, say, 20-30\%, but this is not official ASPECTS and does not remediate its coarseness. \$6.2 uses a probability entropy measure to show that ASPECTS exacerbates CT's own unreliability. ASPECTS's alarming interrater standard deviation converts to an even more alarming loss function. \$6.3 pools MRI data into a scatter plot showing that ASPECTS often misrepresents volume across clinical decision thresholds, sometimes dangerously far. As a rough statistical average, $\geq " 1 / 3$ " MCA best matches ASPECTS $\leq 5$, cutting at about $65 \mathrm{~cm}^{3}$, barely $1 / 4$ of the MCA territory. Attending to a salvageable penumbra, the difference between a hypoperfused zone and an infarct core, many authorities realize that the categorical 10-step ASPECTS concept is untenable.

There has long been an easy, adequate volume estimator- $A B C / 2$, from the spheroid formula $(4 / 3) \pi r_{1} r_{2} r_{3}$ but multiplying diameters instead of radii and approximating $\pi$ well enough as $3(\$ 7)$. This continuous measure obviates ASPECTS's chunkiness, biases, and predisposition to large errors. With modern picture archiving and communication systems (PACS) able to measure ellipse area, $A B C / 2$ refines to $2 S h / 3: 2 / 3$ of an area $(S)$ times the perpendicular diameter $(h)$. For an irregular shape, either one is an approximation requiring a step of estimation - something physicians regularly dobut each takes just 20-30 seconds to improve on ASPECTS. The formerly cumbersome, seemingly more precise summation of individually outlined sectional volumes is becoming available by software such as e-ASPECTS ${ }^{\circledR}$ (Brainomix, without the ASPECTS part) and RapidAI ${ }^{\circledR}$ (iSchemaView), which must start by recognizing acute infarct extent and therefore volume. It would then be aberrant for ASPECTS (by e-ASPECTS ${ }^{\circledR}$ or RAPID ASPECTS ${ }^{\circledR}$ ) to supersede the true volume measurement.

Counterclaims are made that ASPECTS is supposed to be a functionally weighted scale $(\mathbf{\S 8})$ or ASPECTS is required by stroke guidelines or accreditation (\$9). Cerebral functional heterogeneity might justify some functional weighting, but (1) ASPECTS's advocates offered this only as a post hoc rationalization, (2) functional weighting for an infarct core would have to be developed scientifically in a way that ASPECTS was not, and (3) function is best kept explicit as a clinical scale rather than contaminating an infarct volume estimate. ASPECTS has not gained a scientific consensus and makes no appearance in some major stroke trials, nor is it a rigid guideline or an accreditation requirement. \$10 revisits and sums up the bases for this report's conclusion that ASPECTS must (\$6) and can (\$7) be replaced by direct volume measurement to accompany separate clinical scales. 


\section{§2. Ground Rules of Measurement}

A measurement is a comparison, usually thought of as numerical, to a reference, which can be a standardized physical object like a ruler or a weight, a stable physical constant, or a plain number. As a discipline within data theory, our metrology focuses on "problematic aspects of the measurement" rather than the usual suspect, "shortcomings in the substantive model"6 (clinical use of infarct volume). You may, if you wish, postpone this section, jump to ASPECTS in $\$ \mathbf{3}$ or to the data analysis in §6, and electively return to these subsections as the topics they foreshadow come into play.

\section{$\S 2.1$ Scales}

Measurements vary in their amenability to arithmetical operations and statistical analysis. Spatial lengths can be added or subtracted, multiplied or divided, and even squared or cubed. But what would it mean to multiply or divide Celsius degrees, Hounsfield units, or calendar years? From fifth place, what would it take to rise to first place, compared to getting to third?

That measurement scales can differ in kind (beyond just as intensive versus extensive variables) must long have been taken in stride, but it began to be articulated in the $20^{\text {th }}$ century. Bertrand Russell noted that "Some quantities are indivisible" and "Some quantities are infinitely divisible." Charles Spearman mentioned "arbitrary classification," "rank," and "essential measurement." John Maynard Keynes pointed out that "Objects can be arranged in an order, ... without its being possible to conceive a system of measurement of the differences between the individuals."7

In 1946 Stanley Smith Stevens differentiated four successive levels of measurement scales: ${ }^{\mathbf{8}}$

$\begin{array}{ll}\text { Nominal } & \text { Non-gradable determination of sameness (recognizes unordered categories) } \\ \text { Ordinal } & \text { Gradable by determination of order (recognizes greater than or less than) } \\ \text { Interval } & \text { Ordinal plus quantitative differences (based on a unit for interval size) } \\ \text { Ratio } & \text { Interval plus quantitative ratios (based on differences from a true zero) }\end{array}$

The first is inherently discrete (categorical), while the others are increasingly prone to be continuous. Ratios of readings of length, time, weight, or ${ }^{\circ} \mathrm{K}$ make absolute sense without further qualifications. Ratios of readings on scales of ${ }^{\circ} \mathrm{C}$, Hounsfield units (water $=$ " 0 " $\mathrm{HU}$ ), or calendrical years or dates, whose intervals are constant enough, can be made to make sense only relative to some qualification. An ordinal scale ranks objects or concepts in a strict order without fixing the size of an interval. For his barest scale, the nominal, Stevens adhered to an orthodoxy that a numeral must be applied, like assigning numbers to athletes, but they really are just names or ideas without numerical relations.

As a measure of measure, there is more to scale type than Stevens's 4-level ordinal metascale,' but our purposes emphasize this comparison of ratio, interval, ordinal, and nominal scales.

\footnotetext{
${ }^{6}$ Jacoby 1991, p. 6; p. 3: "Data ... as the subject matter of theory"; p. 81: "the parameters of any measurement model ... should be subjected to empirical testing," as in $§ 6$.

${ }^{7}$ Russell 1903, pp. 160-1; Spearman 1904, p. 81; Keynes 1921, p. 21.

${ }^{8}$ Stevens 1946; cf. Stevens 1958 with a more thorough table for mathematical group structure, statistics, and examples.

${ }^{9}$ Mosteller and Tukey 1977, pp. 89-92; Velleman and Wilkinson 1993.
} 


\section{\$2.2 Purpose}

The prior section addresses "the meaning of measurement" relatively concretely, mathematically. But "Every measurement has a purpose,"10 too. In medicine we find several categories of purposes:

1. Is this measurement sufficiently within the range of normal variation so as to not contradict the null hypothesis of normality and not raise concerns? Or else how seriously abnormal is it?

2. Has this measurement changed in time or space? The inevitably related antecedent question is: What is the measure that makes a comparative measurement useful?

3. Does this measurement accommodate the cargo or otherwise exceed some threshold? Or: Does this measurement fit into some space or otherwise remain under some threshold?

4. Readers or officials want a number, so I'll give them one.

Category 4 is hardly worthy of medical science and should be referred back to categories 1-3. A serviceable measure of infarct volume fulfills category 1 (anything $>0$ ) and goes on to examine infarct penumbra or evolution in category 2 and to support thresholding for therapy in category 3 . There may well be other ways to think of purpose, but it definitely should be thought of.

Beyond the measurement as a number, we must contemplate the measure (the "metric") foundationally what is being measured to serve our purpose. For example, Stephen Jay Gould wrote that "measuring intelligence as a single quantity" is "The Mismeasure of Man," " meaning that IQ testing is, worse than just miscalibrated, not appropriately "the measure of the man"12 at all. As another example, dural rather than bony spinal canal caliber is a better measure for spinal cord safety. CT lucency and MRI restricted diffusion compete unequally as measures of infarct extent. The choice of measure and the craft of measurement interact in each of the categories 1-3.

The measurement of infarct volume might seem to be mundanely a matter of just a number. But volume is of interest really as a surrogate for its influence on outcome, especially in terms of risks and benefits for therapies. For volume, ASPECTS is in turn a surrogate (\$2.5). Surrogacy compounds questions of measurement with questions about the appropriateness of the surrogate measure.

This report tackles ASPECTS as a measurement (calibration) and a measure (appropriateness). The discussion plays out against background questions of whether, or in what way (by what curve) and to what useful purpose, infarct volume or anything related to it is a measure of an infarct's risk for symptomatic hemorrhage and of the benefit and risk of early infarct therapy.

But first we must understand more ground rules of measurement.

10 quoted respectively: Stevens 1946, p. 677; Nicholas \& White 2001, p. 3 (cf. Biomarkers Definitions Working Group 2001, p. 92).

11 Gould 1981, p. 20 and title; 1981/1996, p. 52 and title (per 1996, p. 20, to parody "the doctrine of the great philosopher Protagoras that man is the measure of all things"-Plato c. 367 BCE, 160D, p. 73). (Cf. Krutch 1954: "The Measure of Man").

12 Steinbeck 1954, pp. 19-20: "What have I contributed in the Great Ledger?... the quality of his gift is the measure of the man." He is contemplating the self-imposed measure itself as "man's greatest curse, and perhaps his greatest glory." 


\section{\$2.3 Precision and Accuracy}

Precision and accuracy are definite (precise) concepts requiring meaningful (accurate) use. Here we define them for numerical use. "Reliability" can be a pragmatic term entailing both, ${ }^{13}$ a popular term for repeatability, or a weasel word to evade clarity about precision and accuracy.

Precision is framed internally in terms of the measurements themselves. It operates at two levels under related definitions. (The numerical description can be generalized to qualitative categories.)

A formal (by form or appearance) definition of precision $_{1}$ uses the number of significant figures or decimal places expressed. Allowance must be made for the numerical base used-e.g., 2 versus 10. Rather than defining the precision of a measurement, this formality defines the precision of the expression of a measurement. An upper bound on precision $_{1}$ is a scale's readout (digital or analog) as augmented by the user's ability to interpolate (if analog) or opportunity to average readouts.

An operational definition of precision $_{2}$ is repeatability ${ }^{14}$ (an inverse of dispersion or spread, \$6.2). To talk about repeatability we need an expression for it, which depends on the precision $_{1}$. For any numerical base, to gauge the precision $_{2}$ we need enough digits to cover the repeatable values, perhaps out to a digit whose value is less repeatable but better than fully random. The precision $_{2}$ is limited by the object's own nature, the measurement sensor system, and the care taken in using it.

The points of the repeatability definition of precision $_{2}$ are that (1) digits (or precision ${ }_{1}$ ) utterly beyond the level of repeatability are not really measurements and are not usually worthwhile, (2) an upper bound on precision $_{2}$ is random error ( $\approx$ random noise), and (3) precision $_{2}$ therefore is an approximate upper bound on how detailed a measurement expression should be ( precision $_{1}$ ). The smallest meaningful digit-place, or a statistical measure like standard deviation in the same units, indicates absolute precision . Dividing this by the measurement gives relative precision $_{2}(\%)$. The precision $_{2}$ (and precision 1 ), paid for and adopted, must have a purpose (\$2.2) by which we judge whether we have attained sufficient precision or are overpaying for too much.

Accuracy respects the higher external truth of scientific realism, something that we can get wrong. In the finest analysis, though, truth (or validity) is often a fuzzy set ${ }^{15}$ with an intrinsic imprecision ${ }_{2}$. Accuracy primarily describes an expression within its precision $_{2}$ - that it be not repeatably wrong. Precision $_{1}$ has its own aspect of accuracy, in that extra digits overstate precision $_{2}$ (although when calculated some may be kept as tags). Accuracy is a goal, and precision is one of its servants.

A barrier to accuracy is systematic error ${ }^{16}$ - a biased mean or perhaps any non random noise. Yet even without biasing a measurement's overall expected accuracy, imprecision ${ }_{2}$ still degrades the individual accuracy that matters to a patient (\$6). Medical care tends to excuse measurements of low precision $_{2}$ as probably accurate enough, often enough, but this does not suit all purposes.

13 e.g., Hayes \& Krippendorff 2007, p. 78: "kinds of reliability—stability, reproducibility, and accuracy...."

${ }^{14}$ Kirkup \& Frenkel 2006, p. 34: repeatability refers to "measurements ... under (as far as possible) identical conditions"; reproducibility refers to "at different locations ... consistency between values obtained by different experimenters."

15 Zadeh 1965, p. 339: "a fuzzy set ... a class with a continuum of grades of membership." Even as a matter of degree, "A test has validity if it measures what it purports to measure"-Allen \& Yen 1979, p. 95.

${ }^{16}$ Kirkup \& Frenkel 2006, p. 33: "if a systematic error has not been accounted for, all the values could be misleading." 


\section{\$2.4 Getting It Wrong-The Loss Function}

A loss function (Fig. 2.1) measures the harm caused by deviation from an optimal decision. ${ }^{17}$ A measurement's loss function quantifies harm by degrees of inaccuracy (versus the truth, §2.3), unless, preliminary to truth, the harm is seen as disagreement of measurements with each other $(\mathbf{\$ 5})$. The loss function concept is familiar in estimates we make, based on common and professional sense, to minimize harm and risk. It blends objective and subjective criteria, making it intuitively sensible yet hard to quantify rigidly. ${ }^{18}$ Issues that arise in choosing a loss function are asymmetry, nonlinearity, and variability in time or space (nonstationarity) or for different evaluators (matter of opinion).

A quantitative measurement's loss function distinguishes positive and negative deviations whose disutilities typically are asymmetric..$^{19}$ From a busy person's optimal arrival time at airport security, 10 minutes late is worse than 10 minutes early. Other models of asymmetry include nail clipping (Ow!) and The Price Is Right. Physicians weigh underdiagnosis against overdiagnosis asymmetrically.

Over a wide range, a loss function may resemble sigmoid growth $\left(\right.$ logistic $\left.{ }^{20}\right)$ curves outward both ways from the optimal $x=0$ (Fig. 2.1C). Proximally there is tolerance without appreciable harm. With more deviation the harm escalates to steeper slope until further deviation becomes irrelevant and the curve levels off. ${ }^{21}$ The lower part of the curve, where we strive to remain, is supralinear: twice the deviation $x$ incurs more than twice the harm $y$. In this part, $y \approx|x|^{n}$, where $n>1$ ( $n=1$ is linear). This convex (-down) curvature is often approximated as quadratic, where $n=2$. (For a gain function to the right of $x=0$, the concave upper segment, now favored, describes a law of diminishing returns.) The loss is a function for individual decisions, but $\$ 6.2$ derives a quadratic mean loss function for a series of alternative decisions, then to be compared among series.

In medicine, e.g., in laboratory values and medication dosing, we typically have asymmetric, convex loss functions, which vary by the patient's brittleness, and lots of differences of opinion. For strokes we consider that a relation (function) exists whereby volume error $(x)$ incurs probabilistic (not deterministic) harm, for which we estimate a loss function with $n \geq 1$. A kappa (א) coefficient of interrater agreement (\$5) for a scale like infarct volume, whose loss function rises $(n>0$ for $|x|>0)$ for increasing degrees of disagreement, must be weighted. If an infarct's probability to bleed and the conditional probability of any given bleed to be symptomatic both rise linearly with volume, then the loss function is supralinear. With supralinearity, larger errors in infarct volume estimation, and choosing the wrong kind of $\boldsymbol{\kappa}$ for research, become disproportionately harmful.

${ }^{17}$ Wald 1950a, pp. 232-3: "the relative degree of preference we would have for the various possible final decisions $d$ if some [state] were known to us to be the true [state] ... described by a non-negative function ..., called loss function," same as Wald 1950b, p. 8: “... called weight function” for what Wald 1949 (pp. 165, 172) already called "loss" (sharply distinguished from "cost” and "risk" functions by Wald 1950b, p. 12); Painsky \& Wornell 2018.

18 Henning \& Kutlukaya 2007, pp. 21, 36-7.

19 e.g., Cohen 1968, p. 219, mentioned for "different costs" reflecting the loss function underlying his weighted $\boldsymbol{\kappa}$; Zellner 1986, particularly pp. 446 (asymmetry), 451 (quoting a referee on "choice of a loss function").

${ }^{20}$ Verhulst 1845, p. 8 ("logistique").

21 Henning \& Kutlukaya 2007, p. 26. This is where it really can't get any worse, or at least it no longer matters. 
Figure 2.1: Some examples of loss functions for quantifiable deviations along the $x$-axis. Loss is graphed as a positive quantity ( $y$ axis). In A and C, the loss is maximally " 1. "

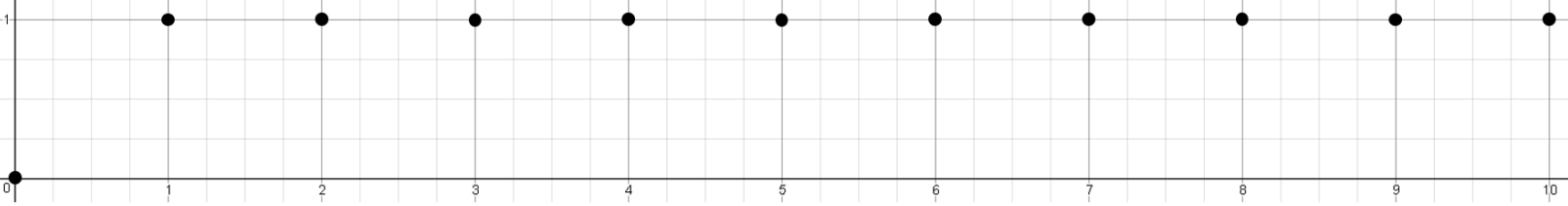

A. Flat unilateral loss function for discrete deviations: anything other than all right is wrong. In a phone number's loss function per wrong digit, the loss is that a wrong number is dialed. This loss function considers neither the probability of misdialing each digit position nor the degrees of embarrassment for different outcomes on the other end of the line.

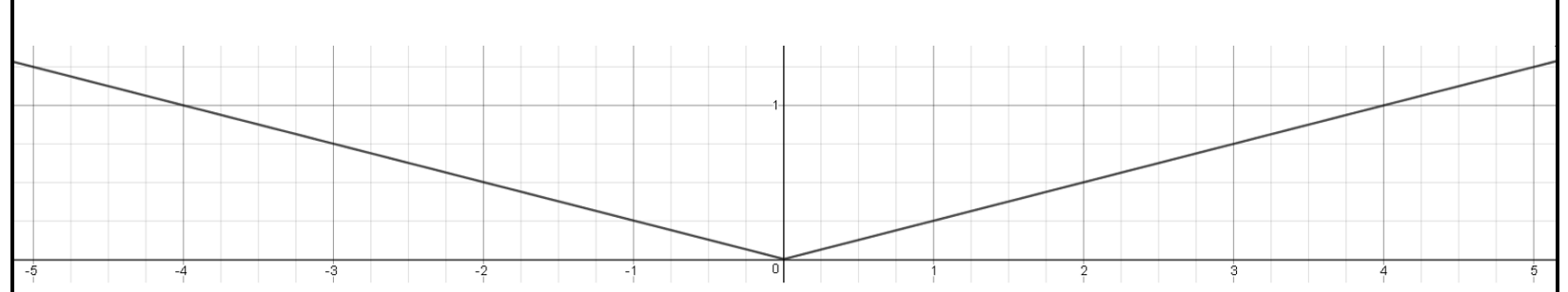

B. Symmetric linear loss function: an idealized model with limited real-life applicability.

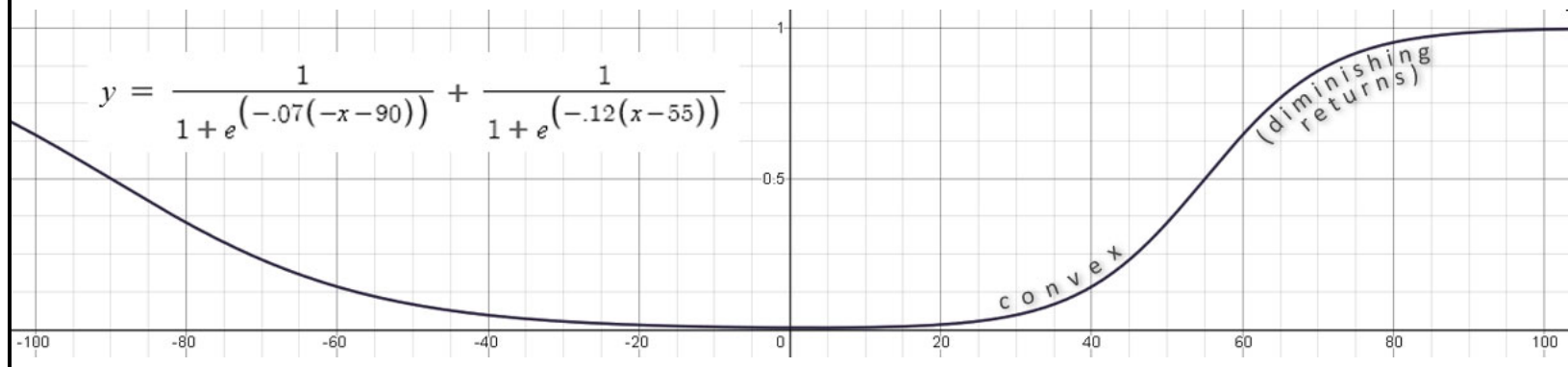

C. An asymmetric, bilaterally logistic, loss function for $x$ as a deviation from the true volume adds two logistic curves of the form $y=1 /\left(1+e^{\wedge}\left(-k_{\text {steepness }}\left(x-x_{\text {atinflexion }}\right)\right)\right)$ with $y \approx 0$ at $x=0$. The value or harm $y$ could be in arbitrary units or a probability maximal at 1 . Each curve is convex from $x=0$ to the inflexion point. The curve for $x>0$ suggests the stochastic harm of overestimating an acute infarct volume. Overestimation increases the probability to exceed a treatment threshold and fail to help a patient who could be helped (covert harm). Underestimation $(x<0)$ increases the probability to cross wrongly below treatment threshold and waste effort or cause overt harm (cf. Table 6.3 Comments). This $y=f(x)$ is a debatable subjective function in professional judgment and is shown only as an illustration.

The grids are from, and the B \& C curves were plotted in, https://www.desmos.com/calculator. 


\section{§2.5 Correlation and Surrogacy}

Like loss function, correlation is a primitive intuition recently set to the tune of mathematics. ${ }^{22}$ Statisticians like to narrow "correlation" to the product-moment correlation coefficient, $r$, or else the rank-order correlation coefficient, $r_{\mathrm{s}}{ }^{23}$ which are our concerns.

A basic question is whether a relation is deterministic, or else to what degree it is interrupted by systematic or chance variation - kinds of noise - perhaps to the point of independence. We use correlation as a nondeterministic concept primarily to distinguish a relation from no correlation. It does not take much correlation to make a correlation, and just saying there is one says little. Moreover, a given $r$ can have graphs in a wide range of shapes, ${ }^{24}$ of varied usefulness.

Correlation supports scientific investigation through surrogacy. ${ }^{25}$ For hypertensive hemorrhage, a genuine hazard in itself, its correlation with high blood pressure makes the latter its surrogate. Office readings are a second-order surrogate, correlating with the time-integral pressure surrogate. We might like to assume correlation is transitive ("The Transitivity Misconception"26), but think about white-coat and masked hypertension. ${ }^{27}$ Correlations are typically rough, showing at best short-range transitivity and declining value when still positive at all, and often instead becoming 0 or inverse. ${ }^{28}$ Correlation excuses surrogacy only when the target is inaccessible or impractical to use.

Multiple surrogates may be further helpful, depending on their curves and mutual independence. To the degree that surrogates correlate with (or derive from) others, they add less value to the pool. Selection among multiple surrogates may be optimized by principal component analysis, ${ }^{29}$ whereby some may be found to truly add nothing to the target's assessment.

As chance blurs correlation, exceptions get excused and may not be tracked well enough to realize that a correlation is inadequate. Correlation, like average, is an unreliable index for individualseasily overrated as grounds for a surrogacy. It is best whenever possible to look past correlation to the variable of real interest or at least to its most proximate available surrogate.

22 Galton 1877, p. 532: "r," initially for "reversion," later "regression," a forerunner of correlation with its use of $r$; Galton 1888, p. 135: "co-relation," initially to distinguish it from "correlation" in its older sense of inextricability, soon "reverted to correlation" (Stigler 1986, p. 298); Pearson 1895, p. 241: $r$, "co-efficient of correlation (Galton's function)"; Pearson 1896, pp. 264-5: "the best Value of the Correlation Coefficient ... what is practically the best method of determining $r \ldots \sum(x y) /\left(n \sigma_{\mathrm{x}} \sigma_{\mathrm{y}}\right) \ldots$ the product-moment" (notation slightly modernized, $x, y \equiv$ deviations from means, $\sigma_{\mathrm{x}}^{2} \equiv n^{-1} \sum x^{2}$; cf. Pearson 1920, pp. 28-9; cf. next, p. 77); Spearman 1904, pp. 78-82, 86-8, "Method of rank differences," R, now $r_{\mathrm{s}}$ or $\rho$. Agreement (\$5) is a stricter measure, possibly absent even with perfect correlation (e.g., Krippendorff 2004a, p. 244).

${ }^{23}$ Kendall \& Buckland 1957, p. 66. Both $r$ and $r_{\mathrm{s}}$ are product-moments - for interval and ordinal scales (\$2.1) respectively.

${ }^{24}$ Anscombe 1973, pp. 19-20: an example of the same $r$ from four datasets with very different graphs.

25 e.g., Saver et al. 1999, p. 293: "surrogate end points," basically meaning one variable serving for another (cf. §2.2).

${ }^{26}$ Castro Sotos et al. 2009, title. Lacking transitivity, correlation is not an equivalence relation. Cf. $\$ 4$ pp. $26-8$.

27 e.g., Townsend 2018 - i.e., especially in relation to integral hypertension and to the latter's real medical risks.

${ }^{28}$ Langford et al. 2001 gives a vector-addition analogy showing visually how wrong chain-correlation can be.

${ }^{29}$ Pearson 1901; Fabrigar et al. 1999, p. 275 (“data reduction"); Armitage et al. 2002, pp. 456-63. 


\section{§3. The Development of ASPECTS}

\section{\$3.1 ASPECTS Invented}

ASPECTS, the Alberta Stroke Program Early CT Score, is a point-scoring system for acute MCA infarct volume estimation. Its founding article by Barber et al. ("for the ASPECTS Study Group," located mostly in Calgary, Alberta), appeared in The Lancet in 2000 ${ }^{30}$ Approximately the same group followed with a second article in the American Journal of Neuroradiology in 2001. ${ }^{31}$ Discerning the motive and modus operandi of ASPECTS requires close readings of these articles. We discuss the neurological motive here and leave the details of ASPECTS for \$3.2.

Barber et al. accepted or originated the following propositions: ${ }^{32}$

(1) A systematic review of 17 clinical trials suggested that thrombolysis ... may increase the proportion of patients with stroke surviving and able to live independently. ${ }^{33}$

(2) [The same systematic review] suggested that ... thrombolysis [is] associated with an increased risk of symptomatic intracerebral haemorrhage. ${ }^{34}$

(3) The most convincing evidence for the efficacy of alteplase comes from the National Institute of Neurological Disorders and Stroke (NINDS) study, which randomised patients within $3 \mathrm{~h}$ of stroke. ${ }^{35}$

(4) However, there is uncertainty about who to treat - for instance, the elderly, patients with severe strokes, and those with early ischaemic change on computed tomography (CT).

(5) Concern has arisen about the reliable detection of early ischaemic change on CT and of [= about] its [= early ischaemic change's] significance in relation to $[a]$ functional outcome and $[b]$ the risk of symptomatic haemorrhage before [= after] the administration of thrombolytic therapy. The European Cooperative Acute Stroke Study (ECASS) trials identified the importance of early CT ischaemic changes in predicting benefit with intravenous thrombolysis. ${ }^{36}$ [my clarifications]

(6) [In ECASS,] Patients were eligible only if there was CT ischaemia involving less than a third of the distribution territory of the middle cerebral artery. This method is not reliable, however, and even experienced stroke clinicians have difficulty in recognizing and quantifying such changes by currently available methods. ${ }^{37}$ [That should have been “... involving $a$ volume less than ...."]

\footnotetext{
30 Barber et al. 2000 (Barber PA, Demchuk AM, Zhang J, Buchan AM).

31 Pexman et al. 2001 (Pexman JHW, Barber PA, Hill MD, Sevick RJ, Demchuk AM, Hudon ME, Hu WY, Buchan AM; http://www.ajnr.org/content/22/8/1534, open access).

32 taking the following quotes from Barber et al. 2000, p. 1670.

33 citing Wardlaw et al. 1999a (a Cochrane Systematic Review).

34 originally intertwined with (1) under the same citation of Wardlaw et al. 1999a (which is verified as supporting both).

35 citing Marler et al. 1995.

36 citing Hacke et al. 1995 and Hacke et al. 1998 (ECASS I and II).

37 citing: von Kummer et al. 1997; Dippel et al. 1997 (an abstract superseded by Dippel et al. 2000 which is cited by Pexman et al. 2000); Schriger et al. 1998; Grotta et al. 1999; and Wardlaw et al. 1999b; all five are discussed in \$4.
} 
ASPECTS's premises began with the competing benefit of thrombolysis and risk of symptomatic intracerebral hemorrhage (sICH). ${ }^{\mathbf{3 8}}$ Thus (1) and (2) jointly targeted sICH while (3) emphasized (1). In (4), "uncertainty about who to treat" brought in CT, and (5) expanded CT's role to predict two things: the original problem of sICH, and now "functional outcome." The latter echoed the ECASS report's remark that the "sign" of "early hypodensity in more than one third of the MCA territory ... is known [to be] associated with a severe stroke and poor outcome and with a high frequency of spontaneous hemorrhagic transformation of the infarct." ${ }^{, 39}$ Thus both hemorrhage and poor outcome in general had already been tied to the volume criterion that became known as the $1 / 3$ MCA rule. Barber et al., citing ECASS, did not dispute this criterion or suggest any other criterion. The $1 / 3 \mathrm{MCA}$ volume rule was Barber et al.'s implied and all-but-stated target to support, and the "currently available methods" (of its measurement) were the target for replacement by ASPECTS in the service of this criterion.

Yet there might seem to have been an ambiguous opening to something else in (6), via "method," because there were two methods. First came the 1/3 MCA rule itself as a method of patient selection. The second was the method of implementing the $1 / 3$ MCA rule by knowing what is the MCA territory, what is its volume as a denominator, and what is the infarct volume as a numerator. Since this latter method was unspecified and left up to the diverse practitioners in diverse specialties and countries, it would be a wonder if it had been reliable. The "difficulty in recognizing and quantifying" is a difficulty of implementation. No challenge to the ideal of the $1 / 3$ MCA rule itself was stated or implied.

Barber et al. followed with a vague mission statement and a hypothesis with more mission: ${ }^{40}$

(7) A system is needed to improve the general reading of CT scans. 9 The main aim of our study was to assess the validity, reliability, and usefulness of a standardised quantitative CT grading system, the Alberta Stroke Programme ... (ASPECTS), in acute anterior-circulation ischaemic stroke.

(8) We hypothesised that by quantification of early ischaemic change detected on CT scan before the administration of alteplase, outcome in terms of independence, dependence, and symptomatic intracerebral haemorrhage could be predicted. [Comment: The complementary pair of outcomes, independence and dependence, reiterates and seems even to prioritize the mission expansion that began to be mentioned in ECASS and in (5).]

The "quantitative" idea in (7) and (8) must have been the only biomarker in view: infarct volume as the $1 / 3$ MCA rule. Improving "the general reading of CT scans" would yield more accurate volumes. There was no offer to improve on ECASS's 1/3 MCA selection criterion. From what is written above, and more below, it is clear that Barber et al. doubted the reliability of estimating this volume fraction of MCA territory, not the appropriateness of an accurate volume fraction if it could be obtained.

38 Fiorelli et al. 1999: defining hemorrhagic infarction as HI1 = small petechiae vs HI2 = more confluent petechiae, vs parenchymal hematoma as $\mathrm{PH} 1=\leq 30 \%$ of infarct vs $\mathrm{PH} 2=>30 \%$ of infarct (writing "area" but meaning volume?), "In contrast to ... other subtypes ... PH2 significantly increased the risk of early deterioration and 3-month death" (p. 2283). Ospel et al. 2021 a find both types with mass effect (PH1 and PH2) "independently associated with outcome" (p. 407). Trials generally use their own circumstantial clinical criteria for sICH, e.g., Goyal et al. 2016a, Supp. Appx. p. 1.

${ }^{39}$ Hacke et al. 1995, p. 1022 (emphasis added). Buchan 2001, p. 99, confirms CT's other motivation, "to select patients who are more likely to benefit from the intervention"-still focused on the "one-third MCA [volume] rule."

40 taking the following quotes from Barber et al. 2000, p. 1670, with emphasis added. 
Summing up Barber et al.'s findings for variably 156 or 154 patients (quoting only as marked): ${ }^{41}$ Symptomatic hemorrhage occurred in 9/65 with ASPECTS $\leq 7$ but just 1/91 with $\geq 8$. ASPECTS between 8 and 7 "sharply discriminated" a large difference in dependence (Rankin 1-2 versus 3-5) but not in death (Rankin 6).

Comparing ASPECTS $\geq 8$ vs. $\leq 7$ to the $1 / 3$ MCA rule as then implemented, ASPECTS had better interrater agreement $(\boldsymbol{\kappa}, \mathbf{\S 5})$ and discrimination for Rankin 1-2 vs. 3-6.

Thus among intravenous tPA-treated patients, they detected three correlations (with: $a$, dependence; $b$, death; $c$, hemorrhage) and found two correlations (with: $a$-or- $b ; c$ ) improved by ASPECTS. But their small sample's implausibly sharp discrimination did not hold up in larger studies. ${ }^{42}$

Citing ECASS's $1 / 3$ MCA rule and "the importance of assessing early CT ischemic changes to predict the benefit from intravenous thrombolysis," Pexman et al. (\$3.2) reiterated that "even experienced stroke physicians and radiologists have difficulty recognizing and quantifying these changes." ${ }^{43}$ The issues for them, Calgary authors again studying ASPECTS, thus remained infarct volume and interrater agreement ( $\boldsymbol{\kappa})$ - i.e., comparing the two methods to estimate infarct volume.

ASPECTS's narrowing of "anterior-circulation" to the MCA territory ${ }^{44}$ reflected a misreading of ECASS I \& II, which, for intravenous tPA, broadly investigated "hemispheric stroke." ${ }^{, 5}$ For ECASS's $1 / 3$ "MCA" volume [equivalent] measure, the variable MCA borders could mean significant infarct volume is really in ACA, AChA, or PCA branch territories and we might not know the differenceor we might know but ASPECTS tells us to ignore these components or pretend they are MCA (\$3.2).

Barber et al. introduced the template for much (but not all, e.g., §6.2, \$6.3) of ASPECTS research:

(a) demonstrate that ASPECTS correlates $^{46}$ with and thereby statistically predicts something (\$2.5), transitively from its correlation with the less biased, more direct surrogate, infarct volume;

(b) refrain from comparing ASPECTS to infarct volume itself using an actual measure thereof, but compare it instead to the straw $\operatorname{man}^{47}$ of unspecified methodology or, in later research, nothing $(\S 4)$;

(c) praise ASPECTS $(\$ 10)$.

\footnotetext{
41 Barber et al. 2000, p. 1672, Figure 2 and Table 1 (156 for hemorrhage; viewable in their article at academia.edu).

${ }^{42}$ Hill \& Buchan 2005, p. 1309 (936 patients); Weir et al. 2006, p. 517 (825 patients, p. 516).

${ }^{43}$ Pexman et al. 2001, p. 1534, repeating the five references given by Barber et al. 2000, p. 1670, to be discussed in $\$ 4$.

${ }^{44}$ Barber et al. 2000, pp. 1670-1, even as they label the (excluded) ACA territory, “A = anterior circulation” (no hyphen).

${ }^{45}$ I: Hacke et al. 1995, p. 1018; II: Hacke et al. 1998, p. 1246. Their "protocol violations" (I, p. 1019: "because of extended early infarct signs"; II, pp. 1245: "with extensive ischaemic changes") did not reference "MCA." But other wording ("more than $33 \%$ of the MCA territory," "exceeding a third of the middle-cerebral-artery territory"), too literally "MCA" instead of volume equivalent, may have been taken (mistakenly) to narrow the entire scope to the MCA territory.

46 e.g., from titles, “ASPECTS correlates”: Beslow et al. 2012; Honarmand et al. 2013; Luvizutto et al. 2015. Most articles are not so concisely quotable, but that is what they show—cf. \$4: Fig. 4.1 (on correlation) and Table 4.1.

${ }^{47}$ Students of the University of Michigan 1877, p. 8: "Into the straw man's mouth are put impossible propositions, and when he has been made to utter them, the speaker proper proceeds to show what an utter ass this straw man is."
} 


\section{\$3.2 ASPECTS Taught}

There is no simple, sensible, complete way to explain ASPECTS. §3.1's article by Pexman et al. is often cited as an instructional guide (e.g., by a 22-page "State-of-the-Art Imaging of Acute Stroke" ${ }^{\text {"48 }}$ ). It is cited in an acute-stroke CT report template in one medical center, but at a neuroradiology journal club there on ASPECTS, of 19 faculty and fellows present, only one admitted having read it. Here we try to unpack this article, including by reference to a table and two figures in it.

The authors said ASPECTS was developed (without saying how) by six physicians:

The six physicians who developed ASPECTS answered a questionnaire on precisely how they interpret and use ASPECTS.... Three neurologists specializing in stroke and three neuroradiologists involved in ASPECTS were interviewed and completed a questionnaire concerning their individual methods of interpretation (Table 1). ${ }^{49}$

This questionnaire's results are discussed below, where the developers did not coordinate well. Based partly on these results, the article claimed to ...

explain how ASPECTS is used in practice, illustrate the method with examples, define landmarks and definitions, show why it can be used on CT scans obtained on all commonly used axial baselines, and emphasize that clinicians from different specialties and with varying levels of experience in assessing acute ischemic $\mathrm{CT}$ changes can agree on an acceptable level. ${ }^{50}$

As the article defined it, ASPECTS originally assessed MCA involvement ...

from two standardized axial [10-mm] CT cuts (Fig 1), one at the level of the thalamus and basal ganglion and one adjacent to the most superior margin of the ganglionic structures, such that they were not seen. On these two sections, which were, by definition, not continuous, the MCA territory was allotted 10 points. ${ }^{51}$

We who work in the field marvel at the idea of "standardized" CT, having never experienced it. Pexman et al.'s Fig 5 (p. 1539) showed the supposed positions of the standardized cuts-the higher (longer) two solid and two dashed lines superimposed on the sagittal cranial outline - at their supposed extremes of angular variation (resembling the common difference between CT and MRI angulation). Referring to the anteroposterior distances between these higher or lower sections' $1 / 3$ and $2 / 3$ divisions, the caption claimed that in this "Maximal variation of ASPECTS sections with baseline alteration ... disagreement is not more than $2 \mathrm{~cm}$." But even discounting the difficulty of estimating thirds, $2 \mathrm{~cm}$ can easily create a potentially critical 1 or 2 points' worth of ambiguity in the number of M regions involved (not really clarified by their FıG 1 C-D, linked below). More ambiguities follow.

\footnotetext{
48 Srinivasan et al. 2006, where it is the only ASPECTS article cited (pp. S78-9).

49 Pexman et al. 2001 (http://www.ainr.org/content/22/8/1534), pp. 1534-5, calling out their TABLE 1 on p. 1539. Barber et al. 2000, p. 1673, had earlier disclosed that "Alastair Buchan invented the ASPECTS scoring system."

${ }^{50}$ Pexman et al. 2001, p. 1535.

51 Pexman et al. 2001, p. 1535 (meaning "not contiguous").
} 
In Pexman et al.'s Fig 1 (available online at http://www.ajnr.org/content/22/8/1534, p.1535): $\mathbf{A}$ and $\mathbf{B}$ were copied from Barber et al., ${ }^{52}$ with the addition of lines and shading indicating "the minimal and maximal variations in size of the cortical areas ... chosen by six expert observers." $\mathbf{C}$ and $\mathbf{D}$ compared different axial-section angulations of an idealized MCA territory on the right and left sides. Compare this comparison to that in their FIG 5, p. 1539, and to our discussion above. $\mathbf{E}$ and $\mathbf{F}$ compared large and small variants of the MCA territory. ${ }^{53}$

ASPECTS allotted 10 points to a normal MCA territory. The suspect side was prespecified. ${ }^{54}$ Then "A single point was subtracted for an area of early ischemic change, such as focal swelling or parenchymal hypoattenuation, for each of the defined regions." The regions were labeled in their FIs 1 , A, B, E, F, and its caption, from which the following quote defines their now-standard abbreviations: ${ }^{.5}$

$C=$ caudate head; $L=$ lentiform nucleus; $I C=$ internal capsule; $I=$ insular ribbon;

$M 1=$ ant. MCA cortex; $M 2=$ MCA cortex lat. to insular ribbon; $M 3=$ post. MCA cortex;

M4, M5, and M6 are anterior, lateral, and posterior MCA territories, respectively ....

Other definitions were: hypoattenuation - "a region of abnormally decreased attenuation of brain structures relative to attenuation of other parts of the same structures or of the contralateral hemisphere"; swelling or mass effect - "narrowing of the CSF space due to compression by adjacent structures." They illustrated some definitions with three CT cases.

This article, later called a "Detailed methodology of the ASPECTS," seems to be the closest thing in print to a how-to manual, although it has been supplemented. ${ }^{57}$ A sketchier "calculator" credited to Dr. Philip A. Barber is online at https://www.mdcalc.com/alberta-stroke-program-early-ct-score-aspects. The ASPECTS official website is http://www.aspectsinstroke.com/ $/{ }^{58}$ where among the EdModules at http://www.aspectsinstroke.com/ed the two that would be expected to teach ASPECTS scoring, modules 5 ("Calculating the ASPECT Score") and 6 ("ASPECTS Case SCENARIOS"), have yielded only ": 404 Page not found" for over two years as of this writing even when logged in. The one case shown at https://demo.brainomix.com/case/7\#easpects (requiring registration to get a generic login), has ambiguous features and lacks verification by DWI (diffusion-weighted MRI).

52 Barber et al. 2000, p. 1671; Pexman et al. 2001, p. 1535 is otherwise the source for this paragraph listing A-F.

${ }^{\mathbf{5 3}}$ Territory variability (\$4) has been recognized in recent decades: Waddington 1974; Damasio 1983; van der Zwan 1991.

${ }^{54}$ Barber et al. 2000, p. 1671: "The baseline CT scan was subsequently assessed with knowledge of the side affected"; Pexman et al. 2001, p. 1540: "good to excellent reliability can be achieved with ASPECTS when the stroke symptom side is known."

55 Pexman 2001, p. 1535. Lacunar infarcts are not excluded (Barber et al. 2000, Pexman et al. 2001, Puetz et al 2009, Menon et al. 2011, contrary to Saposnik 2004, p. e7). Puetz et al. 2008 introduce a posterior-circulation pc-ASPECTS ("developed ... a priori based on clinical experience") with its own opening account of 10 points, subtracting 1 point for apparently any involvement of the occipital lobe, thalamus, or cerebellum separately on each side, and 2 each for the midbrain and pons. Wusthoff et al. 2011 introduce and Beslow et al. $2012 \approx$ clarify and further modify a modified pediatric modASPECTS that more sensibly adds points from 0 and reads $\approx$ all territories - also ACA and PCA ( 2 points each at each of the original two ASPECTS slice-levels), thalamus, and \pm cerebellum (?), each bilaterally - up to 32 or 30 points.

${ }^{56}$ Pexman 2001, p. 1535-without acknowledging the pitfalls of pre-existing sulcal and ventricular asymmetries.

57 quoting Demchuk \& Coutts 2005, p. 411; supplemented by Puetz et al. 2009 and Menon et al. 2011, below.

${ }^{58}$ Modi et al. 2012. Beware: http://aspectsinstroke.com/collateral-scoring shows an unrelated collateral scoring system called "mCTA Collateral Flow," mistakenly called “ASPECTS" by Seker et al. 2016 and Potter et al. 2019 (pp. 1726, 1731). 
The major part of their Results was the section "How Different Physicians Interpreted ASPECTS" (but their FIgs 2-4 displayed in it were called out only from the Methods and did not illustrate Results). Their TABLE 1 addressed 7 questions (1-6) asked of their raters, the three stroke neurologists (N) and three neuroradiologists (R) "who developed ASPECTS." The questions involved matters like (2) whether all or just 2 sections were looked at, (4 and 5) the use of "hypoattenuation ... mass effect and/or gray/white blurring," and (6) whether the IC was to be counted according to anterior limb (ALIC) or posterior limb (PLIC) involvement, or only PLIC involvement. For (6), "N1, R2, and R3 would have made the diagnosis if only the anterior limb had been affected." For (2), "N1 and N2 used [only] the two ASPECTS sections in Fig 1." The others are harder to interpret. Whether based on practices or (mis)understandings, all answers split 3:3 or 4:2. ${ }^{59}$ The use of all CT sections (not just 2) and of just PLIC for the IC region have subsequently gone mainstream. ${ }^{60}$ The commercial products e-ASPECTS and RAPID ASPECTS retain the entire IC (with often ACA-supplied ALIC and AChA-supplied PLIC) as the IC region." ${ }^{61}$ Mass effect as an isolated sign ("or") has been dropped. ${ }^{62}$

Against those disagreements, the Results text countered with issues that all observers agreed on:

(a) Involvement of any part of the insular ribbon ("anterior or posterior"), lentiform nucleus ("all or part"), and caudate head ("only part ... had to be obscured") counted as involvement of that region.

(b) "Comparison was always made with the opposite hemisphere" for considerations of "poor [CT] quality, new bilateral infarcts, or one new infarct along with a contralateral old infarct (most likely)."

(c) "All viewers subjectively interpreted a lesion with very marked hypoattenuation as an old lesion."

(d) "All viewers said that they divided the MCA territory roughly into thirds at M4 to M6 levels."

(e) "All ... regarded M1 as anterior to ... the sylvian fissure and included the frontal operculum."

$(f)$ "All observers identified the anterior end of the temporal lobe as the anterior boundary of M2."

$(g)$ "When a CT scan showed that a patient's head was not symmetrically situated in the scanner, owing to either tilt or rotation ..., or both, all observers dealt with this as best they could by trying to compare corresponding areas in the two hemispheres, even though these were on different CT sections."

Points $(e)$ and $(f)$ were anatomically synonymous. At least as much text discussed disagreements on other matters. ${ }^{63}$ Procedural disagreements started this agreement study off on a wrong foot.

\footnotetext{
59 Pexman et al. 2001, pp. 1537-9. Their TABLE 1's “Total in Agreement," bottom, refers only to totals of "Y[es]" per rater.

${ }^{60}$ Barber et al. 2005, p. 1529 (“.. in practice each reader reviewed the entire sequence of slices ....”); Demchuk et al. 2005, p. 2111 (“All axial cuts ... posterior limb ...."); Lin et al. 2008, p. 936 (“All images”); Singer et al. 2009, p. 2744; Puetz et al. 2009, p. 355: "A common misunderstanding of ASPECTS scoring is to assess EIC [early ischaemic changes] only in two standardized cuts ... ASPECTS methodology has quickly evolved since to include the assessment of all axial cuts of the brain CT scan in two standardised levels of the MCA territory"; Broderick et al. 2013, Supp. Appx. p. 22; Hill et al. 2014, Supp. p. 1 ("All," "posterior"); Wilson et al. 2018: "current methods overwhelmingly use the whole scan."

${ }^{61}$ Brainomix (2021); iSchemaView (n.d.); cf. Maegerlein et al. 2019, p. 143. Also in-house systems: Fahmi et al. 2017, p. 119; Kuang et al. 2019, p. 34.

${ }^{62}$ Wardlaw \& Sellar 1994, p. 1934: not defined by mass alone; Na et al. 2005; Butcher et al. 2005; Butcher et al. 2007; Puetz et al. 2009, p. 356, and Menon et al. 2011, p. 408, say: "EIC ... hypoattenuation only"; cf. Hill et al. 2014 , Supp. p. 1.

63 Pexman et al. 2001, pp. 1537-9.
} 
The Calgary group have since updated, saying that "To allow visualisation of all ASPECTS regions in more than one slice, axial cuts with 4-5 mm slice thickness should be used." ${ }^{64}$ ASPECTS no longer relies on just two slices as it may still seem to some. ${ }^{65}$ Thinner slices detect smaller infarcts. Now with multidetector $\mathrm{CT}$ and its near-isotropic multiplanar resolution, intersecting slices can help distinguish infarcts from the illusions of volume averaging and pixel noise. Therefore still smaller infarcts, visible in only one thin slice's thickness, might be confirmed in a perpendicular slice.

Such technical advances highlight the question of how little regional involvement is scorable, since detecting smaller infarcts in, or smaller extensions into, more regions can further inflate scores. Just "an area of early ischaemic change" ${ }^{66}$ sets no threshold above bare detection in any part of a region (made explicit above for the lentiform and caudate nuclei and nearly so for the insula) and this criterion remains official even if it is not universally heeded. ${ }^{67}$ On this and other matters, research and practice are haunted by "differences in ASPECTS determination techniques."

With 12 comparisons "across specialties," Pexman et al. found improved interrater agreements using ASPECTS compared to "the 1/3 MCA rule"69 - confirming that volume and the $1 / 3$ MCA rule were the target of ASPECTS. Since ASPECTS targeted the $1 / 3$ MCA rule, and the MCA territory includes gray and white matter, a scoring system targeting the MCA territory must score gray and white matter. ASPECTS expressly includes white matter (WM) in its IC region. The IC continues the centrum semiovale and corona radiata down between the lentiform nucleus and the caudate (ALIC), third ventricle (genu), and thalamus (PLIC). But since centrum and corona are not part of the IC, they must be scored elsewhere-in the M regions. The M regions are often called "cortical," but "It was stressed that the M5 territory extends medially to the margins of the lateral ventricle." This inconsistency elicits a still closer look Calgary's and some other publications (next page).

\footnotetext{
64 Puetz et al. 2009, p. 356, and Menon 2011, p. 408.

65 e.g., Phan et al. 2006: "The ASPECTS template comprises two slices ... The sum of the weights for the 10 ASPECTS regions was 51\%, indicating that the ASPECTS template accounts for about half of the MCA territory infarct volume" (pp. 477, 480), but it still is not clear whether their missing $49 \%$ was the other slices or the corona radiata. See the following paragraphs.

66 Barber et al. 2000, p. 1671, and Pexman et al. 2001, p. 1535, emphasis added.

67 “abnormal (either partially or completely)” (Lin et al. 2009, p.19); "10 reflects a normal CT scan” (Puetz et al. 2009, p. 356; Menon et al. 2011, p. 408); "ten implies no evidence of new early signs of ischemia in the [MCA] territory" (Hill et al. 2014, Supp. p. 1); "Any detectable" (Herweh et al. 2016, p. 439); "even if the region is only partially involved" (Scheinfeld et al. 2017, slide 12). But "an author once explained ... that he only counts an ASPECTS station ... if it involves at least visually $25 \%$..." (relayed by Lin 2018), and "An ASPECTS region was considered as involved on DWI if the lesion occupied more than 30\% of the respective region" (San Román et al. 2018, p. 897) or 20\% (Qiu et al. 2020, Appx E1, p. 1, cf. note ${ }^{\mathbf{2 4 2}}$ ). Lin 2018 asks, "how does one estimate 25\% reliably?" The de Margerie-Mellon et al. 2013 data as supplied (\$6.3), and as visible in their plot (p.3566), also suggest some raters used a supra-minimal threshold, because 12 patients with infarct volumes of 0.2-15.2 $\mathrm{cm}^{3}$ were scored as ASPECTS=10. Desilles et al. 2017,p. 964: "To be considered as abnormal, DWI hyperintense signal had to be confluent." Schröder et al. 2018 find "low overall agreement" (p.7) between visual scoring and auto-ASPECTS using a $0 \%, 1 \%, 10 \%$, or $20 \%$ involvement threshold. Even if any part seems too aggressive, it is more easily decidable, and no less arbitrary and coarse, than a seemingly fairer intermediate threshold.

68 Padroni et al. 2016, p. 13. Beyond manual methods comes automatic ASPECTS, for which Fahmi et al. 2017, p. 118, describe a detection scheme that differs from human perception and may be hard to understand.

69 This claim of improvement does not hold up — see end of $\$ \mathbf{5}$ for quotes and citations and $\$ \mathbf{6 . 2}$ for additional data.

70 “cortical,” e.g.: Pexman et al. 2001 (pp. 1535, 1539, 1541), Puetz et al. 2009 (p. 355); “... M5 ...,” Pexman, ibid. (p. 1537).
} 
Barber et al. said "For ASPECTS, the territory of the middle cerebral artery is allotted 10 points.",71 There is no cortical specification here, and "cortex" occurs only in their Figure 1 caption where even M4-6 are "anterior, lateral, and posterior MCA territories ...." Pexman et al. (quoted above) repeated this correspondence of "cortex" to "territories" that suggests a correspondence in their meanings.

A matter less of substance than of style is the trope that rhetoricians call synecdoche, which "takes a part and lets it stand for the whole or takes the whole and lets it stand for a part" and "is common in everyday speech."72 Unidirectionally, to semanticists "cortex" serves here as a meronym (partonym), ${ }^{73}$ whose usual neuroanatomical holonym is "cerebrum" but regionally here is "territory," because there also is WM in the cortical-artery territories. The salience of the cortex prompts "cortex" as just a handy meronym to help contrast the final sentences in Barber et al.'s caption: "Subcortical structures are allotted 3 points (C, L, and IC). MCA cortex is allotted 7 points (insular cortex, M1, M2, M3, M4, M5, and M6)." Their "Subcortical" means purely subcortical. If they intended literally a strict subcortical-cortical opposition, "subcortical" would also have to include the MCA lenticulostriate portions of the corona and centrum, vital pathways that can infarct and bleed on their own ${ }^{74}$ If they meant cortical circulations, including the juxtacortical WM that also is supplied by cortical arteries, why exclude other WM? The Calgary group's updates settle it:

Any [acute MCA] ischaemic lesion on axial CT cuts at the level of the caudate head or below will be adjudicated to a ganglionic ASPECTS region (M1-M3, insula, caudate nucleus, lentiform nucleus, internal capsule); ischaemic lesions above the level of the caudate head will be adjudicated to a supraganglionic ASPECTS region (M4-M6). ${ }^{75}$

ASPECTS convexity regions M1-6 are "cortical regions" in that they have a lot of cortex. Knowing not to read "cortex" too narrowly, though some readers and companies still do ${ }^{76}$ we acquit ASPECTS of intending to ignore the corona and centrum. But ASPECTS has other charges pending summed up so far in Table 3.1 and enlarged upon in $\$ 4$ onward.

${ }^{71}$ Barber et al. 2000, p. 1671, with Figure 1.

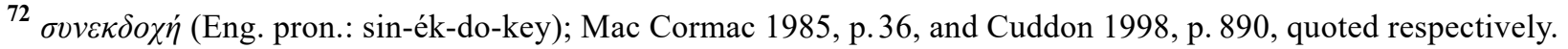
Quintilian c.95, VIII.vi.19, p. 311: "synecdochè has the power to give variety to our language by making us realise many things from one, the whole from the part, ...." Gibbs 1993, pp. 258-9: perhaps synechdoche $\subset$ metonymy $\subset$ metaphor.

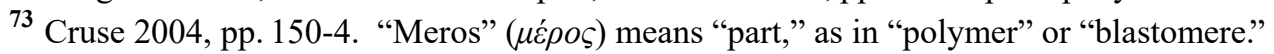

${ }^{74}$ Bogousslavsky \& Regli 1992: In large $(>2.5 \mathrm{~cm})$ pure centrum semiovale infarcts, "the neurologic picture was similar to that found in large superficial or extended (deep + superficial) middle cerebral artery territory infarcts." Lövblad et al. 1997, p. 167: "white matter lesions involving major tracts could produce greater clinical deficits." Ernst et al. 2018, p. 1993: "support the hypothesis of greater impact of the corticospinal tract over cortical lesions." NINDS t-PA Stroke Study Group 1997 shows bleeds that appear to arise in the corona radiata or centrum semiovale: in "Figure 1. All 22 symptomatic ICHs within 36 hours of onset...,"\#1,5,6,7, and possibly some others harder to localize; in "Figure 3. All 21 asymptomatic ICHs during the first 36 hours ...,"\#9, 20, and possibly some others harder to localize.

75 Puetz et al. 2009, p. 355 quoted; Menon et al. 2011, pp. 408-9; but each, in its next sentence, scores caudate body with head.

76 e.g., Farzin et al. 2016 (Supp.p. 5, Fig. e-1), Fahed et al. 2018a (Supp. fig. 1 [same Fig.]), Scheinfeld et al. 2017 (slide 11 ), Kuang et al. 2019, p. 34, Fig. 1, and iSchemaView (n.d.) highlight M-region cortex (the latter three, also juxtacortical WM) while omitting the (deeper) corona radiata. Starting from the earlier prevalence of this misunderstanding, Kawano et al. 2012 "established a modified scoring method, ASPECTS+W, including deep white matter lesions" (p. 2045) in testimony to the importance of their "W" (the WM) in predicting hemorrhage. 
Table 3.1.

The Semantics of ASPECTS

§3 establishes ASPECTS's distinguishing features, principally:

1. Its original citations.

2. Its scoring by units of mixed sizes.

3. Its arbitrary restriction to the MCA territory.

4. Its vague definitions and inconsistent implementation.

5. Its purpose to guide application of the $1 / 3$ MCA rule of infarct volume.

6. Its validation paradigm emphasizing correlations without verifying accurate volumes.

7. Its name.

The name "ASPECTS" needs clarification in order to resolve a misunderstanding. While "CT" is embedded in the acronym, the relation to $\mathrm{CT}$ is not restrictive as a few writers assume, but is only an accident of CT's original availability and convenience. That "CT" itself is the abbreviation for $x$-ray computed tomography is an accident of its 4-year lead over magnetic resonance computed tomography (MRI). Both are tomography, both are computed. $\mathrm{CT}$ is no more an essential feature of ASPECTS nowadays than glass is of eyeglasses or lead is of a pencil lead or modern plumbing. These examples and the presence of "CT" in "ASPECTS" reflect a context-dependent semantic shift or broadening, to the point of "meaning loss or 'bleaching'," as seen in the natural process of grammaticalization. ${ }^{78}$ ASPECT's particulars and problems of anatomic geometry transfer so readily to MRI/DWI (leaving behind CT's intrinsic problems), that writers and readers can look past the "CT" in ASPECTS to denote its specific modality applications as "CT-ASPECTS," "DWI-ASPECTS," etc. "ASPECTS" also lends itself to other schemes with prefixes like "pc-," "mod," and "auto-" (in notes ${ }^{55,67}$ ).

Although this report analyzes data on CT-ASPECTS (\$6.2) and DWI-ASPECTS (\$6.3), it investigates ASPECTS's anatomy (\$6.1) and is conceptually about ASPECTS per se. ASPECTS per se is problematic (1) as a measurement because of its vague (e.g., M1-M6) and inconsistent definitions (e.g., in the capsules and other WM) and its vulnerability to variations in positioning (M1-M6), and (2) in its conception as a measure, even beyond its vagueness and inconsistency, because of its intrinsic region-size heterogeneity and categorical scoring of the regions. These metrological flaws predict erratic performance in measuring volume, which is tested and quantified empirically in $\S 6.2$ and $§ 6.3$.

77 e.g.: Messé et al. 2007, Bivard \& Parsons 2012, Naylor et al. 2017, and Goyal et al. 2019 (implicitly by usage, leaving the authors' understandings unclear); Kallmes et al. 2019 (explicitly "CT"; rebutted by Kaesmacher et al. $2019 a$ ).

78 Meillet 1912, p. 133: "grammaticalisation"; Hopper \& Traugott 1993, pp. 20-4, 68 (quoted), 87. Familiar examples are the evolutions of "going" (moving) + infinitive, and "have" (hold) + participle, into signs of future or past activity.

79 e.g., Barber et al. 2005, Kimura et al. 2008, Butcher et al. 2008, Lin et al. 2008, Kosior et al. 2010 ; and see $§ 6.3$. 
Page 22 ASPECTS, The Mismeasure of Stroke: A Metrological Investigation

\section{§4. The MCA Territory and Its "1/3" Rule}

The MCA territory gets the most strokes, the most studies, some using the 1/3 MCA rule ( $(3)$, and all of ASPECTS's attention. ${ }^{80}$ To see what problem pre-ASPECTS physicians had using this rule, we check its five motivating citations in Barber et al. (cited as 5-9) and Pexman et al. (cited as 4-8):

\#1. von Kummer et al. put 603 ECASS CTs to 3 neuroradiologists and, for $\leq$ vs. $>1 / 3$ MCA involvement, found $86 \%$ 3-way agreement. ${ }^{81}$ Not just pairs, but Three-of-a-Kind! They found a "paradox of high agreement but a low $\boldsymbol{\kappa}$ " (kappa, a kind of value added beyond chance agreement, §5), which they said

can be partly explained in terms of the low prevalence of patients with a large area of parenchymal hypoattenuation.... Feinstein and Cicchetti [cited] showed that $\boldsymbol{\kappa}$ is affected by prevalence and cannot be used as an index of reliability when observer variability is tested without an analysis of how proportionate changes in the marginal total values affect the calculation of $\boldsymbol{\kappa}^{82}$

Their point that their $\boldsymbol{\kappa}$ is confounded tends to nullify their support for the ASPECTS authors.

\#2. Dippel et al. reported readings by staff and resident neurologists ("None was a radiologist"), by whom "The extent of cerebral infarction was estimated by taking the ratio of the area with (subtle) signs and the total area of the MCA territory. A distinction was made between infarction of less or more than $1 / 3$ of the MCA territory." ${ }^{\prime 83}$ For this their $\boldsymbol{\kappa}$ of 0.37 is fairly low, yet it is higher than that for any other elementary observation except the dense MCA sign. ASPECTS would address the elementary observations as a search check-list without numerical scoring (\$10). And area is not volume.

\#3. Schriger et al. asked 103 neurologists, emergency physicians, and radiologists to each review $15 \mathrm{CT}$ scans to see whether they would notice signs of hemorrhage, acute infarction, imitators, and normals. ${ }^{84}$ Despite the paper's subtitle, "Physician Accuracy in Determining Eligibility for Thrombotic Therapy," none of this had anything to do with measuring the volume of anything. Therefore as a reference for the ASPECTS authors' statement that "even experienced stroke clinicians have difficulty in recognizing and quantifying such changes," 85 it supports only "recognizing," not the "quantifying" part on which ASPECTS is held out to compete with the $1 / 3$ MCA rule.

\footnotetext{
${ }^{80}$ Barber et al. 2005, p. 1529: "a stroke isolated to the brainstem or cerebellum would be given an ASPECTS of 10." Besides excluding all involvement of posterior circulation territories, and of the PCA territory even if of fetal origin, in ASPECTS's "Newspeak" (Orwell 1949) "anterior circulation" ironically excludes the ACA and AChA territories. Anatomically the "anterior circulation" (internal carotid) complements the "posterior circulation" (vertebrobasilar)— e.g., Weir 1987 (pp. 143-7), Osborn 1994 (pp. 251-2: “the anterior (carotid) circulation”), Athauda \& Tan 2014, and Streib et al. 2019 (“... Anterior Circulation ...” in title, confirmed by Jovin 2019 as meaning ICA including pericallosal). \$3.1 points out that hemispheric exclusion outside of the MCA territory misconstrues ECASS.

${ }^{81}$ von Kummer et al. 1997, pp. 328. (They dichotomized at 1/3 MCA: "Dichotomy ... division of the members of a population, or sample, into two groups. ... may be in terms of a measurable variable ..."-Kendall \& Buckland 1957, p. 82.)

${ }^{82}$ Feinstein \& Cicchetti 1990 (the internal citation); von Kummer et al. 1997, pp. 330.

83 Dippel et al. 2000, p. 630 — cited by Barber et al. 2000 as a meeting abstract: Dippel et al. 1997.

${ }^{84}$ Schriger et al. 1998, p. 1294. Their article about volume is Kalafut et al. 2000, approximating 1/3 MCA (attrib. to ECASS) by different a scoring system of Frontal, Parietal, Temporal, Ganglionic, and Insular involvement.

85 Barber et al. 2000, p. 1670 quoted; Pexman et al. 2001, p. 1534 with somewhat different wording.
} 
\#4. Grotta et al. put 70 baseline CTs from the NINDS study to 16 neurologists, emergency physicians, and radiology and stroke fellows "assembled from members of ... stroke teams," and found mostly low $\boldsymbol{\kappa}$-statistics on early CT signs of infarction. ${ }^{86}$ But their TABLE 2 shows $\boldsymbol{\kappa}=1.00$ between their two radiologists for "Hypodensity $>33 \%$." They might have underplayed this because of the $\boldsymbol{\kappa}=0.50$ for "Any early CT [sign of] $>33 \%$ " caused by their $\boldsymbol{\kappa}=-0.08$ for "CSF comp[ression as a sign of] $>33 \%$." (Swelling alone, now dropped as a sign of early infarction (\$3.2), is only very subjectively judged and is unreliable without a prior CT because it has other causes — particularly baseline sulcal asymmetry. ${ }^{87}$ ) Against a gold standard (the NINDS neuroradiologist), reviewers' sensitivity and specificity were, respectively, $78 \%$ and $57 \%$ for "any early CT change," and $44 \%$ and $94 \%$ for "early CT findings involving $>33 \%$ of the MCA." ${ }^{, 88}$ Especially noting the instance of $\boldsymbol{\kappa}=1$ for the latter as hypodensity, the greater problem, detection, is beyond help by ASPECTS's supposedly better $\boldsymbol{\kappa}$ s (cf. §5).

\#5. Wardlaw et al. put 12 CTs "from the ECASS trial book of examples of patients scanned within 6 hours of stroke" plus two normals to 15 neuroradiologists, stroke and other neurologists, trainee physicians, and a G.P. Unlike the ECASS, these CTs were not limited by the $1 / 3$ MCA rule, so the volume question could be asked. The physicians identified early infarct signs poorly and were "even worse at judging when an infarct occupies 'a third or more of the MCA territory'.", Again, if physicians poorly recognize the signs at all, then a question of volume estimation is premature.

Grotta et al. cited two more articles of perhaps nominal relevance to ASPECTS's motivation:

\#6. Marks et al. put 50 hyperacute CTs to 3 neuroradiologists. They found $72 \% 3$-way agreement, $60-85 \%$ sensitivity, and $86-97 \%$ specificity, calling this "good to excellent interobserver reliability in the detection of $>33 \%$ or $<33 \%$ hypoattenuation of the MCA territory for neuroradiologists."

\#7. von Kummer et al. put 45 CTs to 6 neuroradiologists, who binned volume estimates in $20 \%$-age point segments instead of dichotomizing at $33 \%$. "Complete pairwise agreement for the identical percentage of MCA territory's being hypodense was rather low, varying from $23 \%$ to $26 \%$. Agreement increased considerably if disagreement by at most one or two categories was allowed." ${ }^{\prime 1}$

Barber et al. indirectly cited, via proposition (1), two other trials that used the $1 / 3$ MCA rule, citing ECASS I: ATLANTIS and PROACT II. These mention CT protocol violations $\left(\$ 3.1\right.$ note $\left.^{45}\right)$, presumably mostly errors in applying this rule,$^{92}$ without statistical measures of agreement.

\footnotetext{
${ }^{86}$ Grotta et al. 1999, pp. 1529-31.

${ }^{87}$ Baseline asymmetries exist and could account for von Kummer et al.'s (1997, p. 328) 1.8\% rate lacking hypoattenuation. When an infarct causes enough swelling to be objectively recognizable without comparison to a baseline, its cytotoxic edema is visible. Failure to heed this rule allowed a patient with something else to get tPA: it was subtle subarachnoid hemorrhage from an aneurysm, making most of the sylvian fissure $\approx$ isodense with cortex (Cherian et al. 2014).

${ }^{88}$ Grotta et al. 1999, p. 1531

${ }^{89}$ Wardlaw et al. 1999b: quoted respectively. p. 651, p. 652.

90 Marks et al. 1999 (p. 391 quoted), cited by Pexman et al. 2001 (p. 1540) calling the agreement "moderate to good"

91 von Kummer et al. 1996 (pp. 1744-5 quoted).

92 respectively: Clark et al. 1999 (p. 2025, only ECASS I\&II's violations), Furlan et al. 1999 (p. 2006, their own).
} 
Barber et al. sought a volume-like threshold empirically and found \$3.1's prognostic function. Their Table 1 compares this to the $1 / 3$ MCA rule and finds identical statistics for symptomatic infarct hemorrhages. But their ASPECTS threshold "sharply discriminat[ing] independence [from] dependence ... at 3 months" and matching the $1 / 3$ MCA rule in its numbers of bleeds, was $\geq 8,{ }^{93}$ which corresponds to a $1 / 4 \mathrm{MCA}$ rule ignoring region inequality and incomplete involvement. Yet, ignoring region inequality, and even having cited Barber et al.'s threshold at $\geq 8$, Pexman et al. instead seem to report $\geq 7$ for their interrater agreement comparison to the $1 / 3$ MCA rule. ${ }^{94}$ Confusion is avoided by denoting a threshold at, say, $\geq 8$ versus $\leq 7$ (or $>7$ versus $<8$ ) as $8 / / 7$. Three groups approximate a $1 / 4$ MCA rule empirically as 70 or $72 \mathrm{~cm}^{3}$ using DWI. ${ }^{95}$ But can we interpret Barber et al.'s threshold at $8 / / 7$ as any volume rule if we do not know what volume any individual abnormal $(<10)$ ASPECTS represents $(\S 6)$ ?

"[D]octors ... may fail to understand what constitutes an infarct in a third of the MCA territory as the boundaries of the MCA territory vary from patient to patient ...."96 One therefore cannot expect sterling $\boldsymbol{\kappa} \mathrm{s}$ on fractional volume estimates or dichotomizations. What volume measurement technique was used? No one said (except \#2: an area ratio), until \#4 divulged "the 'eyeball' technique,"97 which is $n o$ technique. An existing technique (\$7) was ignored. The lack of territory delineation and technique are two more wrong feet — as a classical straw man fallacy — on which Pexman et al. started off $(\S 3.2)$.

An MCA territory's borders and volume are knowable in life only when recognizably the entire territory is infarcted. ${ }^{98}$ Moreover, the probability of hemorrhage is a volume function of infarcted tissue that cannot be contingent on a distant boundary. The denominator MCA territory therefore must be some modal, median, or mean volume of brain tissue. The constructive response has been to stop saying " $1 / 3$ MCA" and to pick a standard MCA volume of which to use $1 / 3$. We have a mean we can use: van der Zwan et al. laid the foundation with simultaneous injections of differently colored Araldite $\mathrm{F}$ mixtures into the ACA, MCA, and PCA. Although each volume varied by a factor of 3.2-3.3, the means were, in $\mathrm{cm}^{3}$ : ACA, 137.8; MCA, 284.4; PCA, 118.7.99 While $1 / 3$ of the MCA average ofsay $285 \mathrm{~cm}^{3}$ — would be $95 \mathrm{~cm}^{3}$, a consensus ${ }^{100}$ picked a memorable $100 \mathrm{~cm}^{3}$ (but see $\$ 6.2$ ).

\footnotetext{
93 Barber et al. 2000, p. 1672. Recall, the sharp discrimination did not hold up (\$3.1).

${ }^{94}$ Pexman et al. 2001: $\geq 8$ vs $\leq 7$ (8//7) in "sharp increase ... ASPECTS of 7 or less" (pp. 1534-5), in "Interobserver reliability for ASPECTS was assessed according to divisions of greater than 7 and 7 or less ..." (Methods, p. 1536), and in "the dichotomization at greater than 7 and 7 or less" (Discussion, p. 1541); but (by confusion?) $\geq 7$ vs $\leq \mathbf{6}$ (7//6) in "ASPECTS dichotomized between 7 or greater and less than 7" (Results, p. 1539) and "< 7 vs $>7$ " in TABLE2 (p. 1540).

95 Šaň́k et al. 2006, Barak et al. $2008\left(70 \mathrm{~cm}^{3}\right)$; Arsava et al. $2008\left(72 \mathrm{~cm}^{3}\right)$. Yoo et al. 2009, pp. 2047-8, by DWI: “ $>70 \mathrm{~cm}^{3}$ were termed the 'futile group'." Gonzalez et al. 2013, p. i10, use $\geq 70 \mathrm{~cm}^{3}$ in an algorithm. Schaefer et al. 2015, p. 263 (FIG 3B) find bad-outcome probability to reach 0.95 at $70 \mathrm{~cm}^{3}$ for left vs. $200 \mathrm{~cm}^{3}$ for right MCA infarcts.

${ }^{96}$ Wardlaw et al. 1999b, p. 653; cf. Roberts et al. 2002, p. 1563, and suggesting use of $60 \mathrm{~cm}^{3}$, or " $\approx 5 \mathrm{~cm}$ " $\left(\approx 65 \mathrm{~cm}^{3}\right)$.

${ }^{97}$ Grotta et al. 1999, pp. 1531-2. Cf. Tomsick 1994, p. 16, comparing infarct "eyeball estimates" to "guesstimation." "[N]o specific criteria to estimate greater than one-third involvement of the MCA territory on the initial CT scan has been published"-Silver et al. 2001 (p. 114), suggesting an "idealized" territory (p. 113). No one knew what volume was being approximated or how well. §6.2 suggests that a raw "1/3" MCA rule averages $\approx 1 / 4$ MCA in practice.

98 Phan et al. 2005, finding mean $195.5 \mathrm{~cm}^{3}$, max $366.3 \mathrm{~cm}^{3}$ (p. 988); cf. Tomsick 1994: “approximately $300 \mathrm{cc"} \mathrm{(p.} \mathrm{16).}$

99 van der Zwan et al. 1993, from Tables 3-5 on pp. 1954-5; cf. maps of territorial variability in van der Zwan et al. 1992, Figures 6-11 and 16 from pp. 932, 934, 935, 939.

${ }^{100}$ Albers et al. 2006, p. 510; Singer et al. 2008; Lin et al. 2011; Schröder et al. 2014, pp. 3583-4.
} 
Citing the ECASS criterion of "less than a third of the distribution of the middle cerebral artery," Barber et al. reason from "This method is not reliable," to "A system is needed to improve the general reading of CT scans." ${ }^{101}$ Pexman et al. mention "1/3 MCA rule" 18 times. ${ }^{102}$ Both sets of authors make it clear that they accept the $1 / 3 \mathrm{MCA}$ volume rule as their target, so it deserves to be sourced.

For the $1 / 3$ MCA rule, Barber et al. cite $\# 1-5$ and Pexman et al. cite $\# 1-6$, above. In $\# 1$, "33\%" originates in Materials and Methods, distinguishing "small" and "large" areas of hypoattenuation without rationale or citation anywhere in the paper, but since their data originated in ECASS, that is the implicit source. ${ }^{103}$ In \#2, "less or more than 1/3 of the MCA territory" appears in Patients and Methods, citing ECASS II. ${ }^{104}$ In $\# 3$, there is, as pointed out above, no mention of $1 / 3$ in any form, or of any volume fraction or criterion. In \#4, "33\%" appears in the introductory material before the Methods, attributed to ECASS, and after the Methods only in reference to its methodological use, where it is attributed also to \#1, above. ${ }^{105}$ In \#5, "one third" appears in the introductory material before the Methods, attributed to ECASS and here also to a Guidelines, and after the Methods only in reference to its methodological use. ${ }^{106}$ In \#6, " $<33 \%$ or $>33 \%$ " appears in Subjects and Methods, and after the Methods only in reference to its methodological use, where it is, again, also attributed to \#1, above. ${ }^{107}$ The other referencesECASS II, the Guidelines, ATLANTIS, and PROACT II—cite ECASS [I].

All citations lead to ECASS. ${ }^{108}$ Its Patients and Methods states the $1 / 3$ rule, but its Comment, that it was chosen to introduce the $\mathrm{CT}$ exclusion criterion of early hypodensity in more than one third of the MCA territory. It is known that this sign is associated with a severe stroke and poor outcome and with a high frequency of spontaneous hemorrhagic transformation of the infarct, gives three citations. ${ }^{109}$ However, not one of these cited articles mentions a $1 / 3$ volume fraction. The $1 / 3$ MCA rule propagated as a "Woozle effect" 110 after ECASS made it up from thin air.

\footnotetext{
101 Barber et al. 2000, p. 1670.

${ }^{102}$ Pexman et al. 2001, pp. 1534-6, 1539-41, 5× in abstract, 13× in main text, sometimes phrased slightly differently.

103 von Kummer et al. 1997, p. 328, referring to Hacke et al. 1995.

104 Dippel et al. 2000, p. 630, referring to Hacke et al. 1998.

${ }^{105}$ Grotta et al. 1999, pp. 1529-32, on 1532 citing Hacke et al. 1995 and von Kummer et al. 1997.

${ }^{106}$ Wardlaw et al. 1999b, citing Hacke et al. 1995 and Adams et al. 1996 "Guidelines for Thrombolytic ..." (citing ECASS).

${ }^{107}$ Marks et al. 1999, pp. 389-92, on 388 citing Hacke et al. 1995 and von Kummer et al. 1997.

108 "ECASS ... introduced the concept of using baseline head CT studies to exclude ... from rt-PA treatment [patients] with ICH and/or major early infarct signs ..."-Patel et al. 2001, p. 2831; "ECASS ... first suggested that involvement of greater than one-third of the MCAT [territory] on early computed tomography (CT) scan was a significant risk factor for hemorrhage and that patients with these findings did not appear to benefit from treatment"-Demaerschalk et al. 2006, p. 201. The NINDS study (Marler et al. 1995) does not mention any such rule or territorial volume or fraction for exclusion, and, based on it, Patel et al. 2001 (with coauthor Marler) find no tPA-treatment interaction by CT early ischemic changes.

${ }^{109}$ Hacke et al. 1995: p. 1018 (P\&M); p. 1022 (Comment), quoted, cites von Kummer et al. 1994 (p. 10 distinguishing "small" from "large" hypodensity by " $50 \%$ of the presumed MCA territory"), Toni et al. 1995, and Toni et al. 1996 ("In press").

110 attrib. by Gelles 1980 (p. 880) to Houghton 1979, from Milne 1926: Pooh \& Piglet follow their own tracks around some trees, suspecting more \& more Woozles ("I have been Foolish and Deluded"-Pooh); cf. Bevan 1953: "Modern Psychologists: Scientific Woozle Hunters?" ("we have been methodologically lax and even naive"-p. 5); cf. Ciaffoni 2017. The Woozle effect is " "evidence by citation' ... when frequent citation of previous publications that lack evidence mislead[s] us into thinking there is evidence"-Straus 2007 (p.230); or "reification-by-accretion"-Houghton 2021.
} 
Fear of a volume-hemorrhage correlation might justify choosing to use a volume threshold, but it does not justify carving one in stone. Although $\$ 2.4$ suggests that the loss function is convex (steeper on the right), it might be statistically indistinguishable from straight as shown heuristically in Fig. 4.1. Indeed, Singer et al. "found a gradual increase in sICH risk with lesion size under $100 \mathrm{ml}$, indicating that there is no clear threshold differentiating between high and low risk," thresholds tend to be statistical artifacts anyway (e.g., \$3.1). The caveat for future reference is that without precise measurements (\$6) we don't even know the shape of the gradual curve.

Figure 4.1 Heuristic hypothetical risk of $\mathrm{sICH}$ as a function of infarct volume.

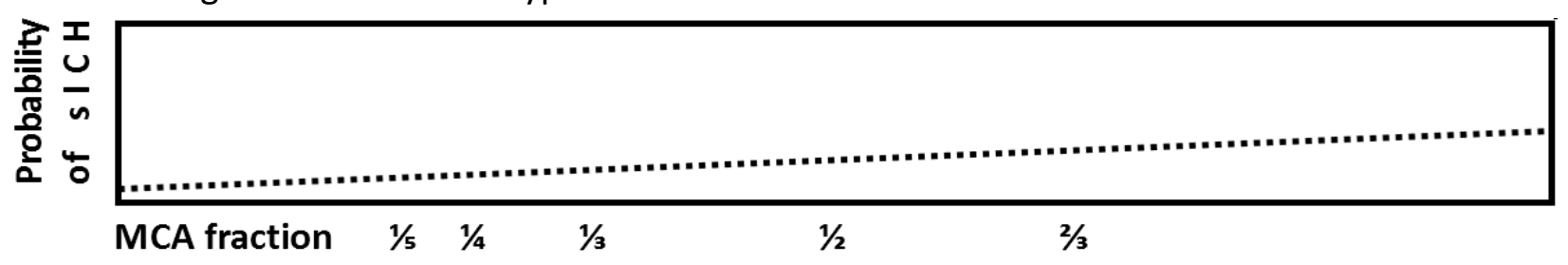

"There is a huge statistical literature pointing to the folly of dichotomizing continuous outcomes."112 An increasing function dichotomized at any of the cuts in Fig. 4.1, if between adequate samples, will approximate a biserial correlation ${ }^{113}$ Then if studied alone it might be claimed to be confirmed as if uniquely true - ordaining for use, e.g., the $1 / 3$ MCA rule ${ }^{114}$ or its surrogate, ASPECTS at 8//7. ${ }^{115}$ Finding a correlation after dichotomizing arbitrarily does not validate any specific dichotomization. Dichotomizing a known gradient to discover a prognostic or treatment correlation begs the question. ${ }^{116}$ Propagating this tautology descends to somewhere between an appeal to authority ${ }^{117}$ and habit. ${ }^{118}$ Such thinking contributes to the supportive tradition that continues to pack the ASPECTS literature, as described and partially listed on the next two pages.

111 Singer et al. 2008, p. 58, continuing, "we did not find a further increase in the sICH rate in the group with DWI lesions exceeding $100 \mathrm{ml}$, possibly because of the relatively small sample size"; cf. Lansberg et al. 2007; both based on DWI.

${ }^{112}$ Senn 2013, p. 207. Many authors note that grouping or dichotomization sacrifices information -also, e.g.: Cox 1957; Agresti 1976; Kraemer 1979, p. 470; Liebetrau 1983, pp. 86-7; Senn 2003; Fedorov et al. 2009; Kenrose 2016; and Senn 2018, p. 621: "Better still, sticking with the actual measurement ...."

113 i.e., not a point-biserial correlation. Kendall 1952, pp. 356-8 (Biserial $\eta$ ); Kendall \& Buckland 1957, pp. 29, 219; Everitt 2006, pp. 47, 304; Corder \& Foreman 2009, pp. 4, 134-149; Kenrose 2016. Cf. more generally \$2.5.

114 above:\#1,2,4,5,6, ECASS, ATLANTIS, PROACT II; and: von Kummer et al. 1997, p. 328; Hacke et al. 1998, p. 1246; Fiorelli et al. 1999, p. 2282; Larrue et al. 2001, p. 440; Patel et al. 2001, p. 2832; Silver et al. 2001, p. 114; Tanne et al. 2002, pp. 1680-2; Albers et al. 2006, p. 509; Lou et al. 2008, pp. 1419-20 ("We also assessed the predictive value of alternative models with different combinations and cutoff points for each of the HAT score variables"-for the $1 / 3 \mathrm{MCA}$ rule); Goldstein et al. 2016, p. 80; Thomalla et al. 2018, p. 613. On the contrary, early on it was admitted that "the cutoff value of DW imaging lesion extension predicting hemorrhage after thrombolysis is still unknown"-Saur et al. 2003, p. 884.

115 Hill et al. 2003a; Barber et al. 2005; Hill et al. 2006; Dzialowski et al. 2006; Singer et al. 2009; Honarmand et al. 2013.

116 circular reasoning, petitio principii, "Begging or assuming the point at issue ..."-Aristotle c. 350 BCE, II, xvi, p. 485.

117 i.e., the species due to lack of diligence: "where ... a correct conception ... is not beyond [the debaters'] competency ... the opinion ... of any person [authority] ... is adduced ... in lieu of ... relevant arguments ..."-Bentham 1824, p. 45.

118 If Pushkin $(1833 / 1964,2 . x x x i .13)$ is right that "Habit ... is given from above ...," an apt paraphrase of his lines 13-14 for the $1 \frac{1}{3}$ MCA rule in stroke trials and therapy would be Привычка свыше намъ дана: Замъна старательности она"Habit to us is given from above: it is a substitute for diligence" (originally ... счастію ..., “... for happiness"). 
An ASPECTS threshold is often tested in isolation for a biological correlation (\$2.5) - with hemorrhagic transformation (HT), clinical severity (CS) or outcome (CO), final infarct volume (FIV), or treatment effect (TE). ${ }^{119}$ The "Woozle effect" (page 25, note ${ }^{110}$ ) here is subtler than with the $1 / 3$ rule because primary sources exist (Table 4.1). But just finding a correlation, or comparing ASPECTS by modality, while ignoring direct correlation by infarct volume, does not yield evidence that ASPECTS would be better than or as good as the volume. Volume works, ${ }^{120}$ probably better than ASPECTS. ${ }^{121}$ Another layer of correlation, ASPECTS, cannot accurately relay the correlations that the truer biological parameter, volume, has with the variables of medical interest, because "correlation is not transitive." 122 \$7 reviews a long-known practical, effective, cheap, quick volume measure, readily available and needing no surrogate. Some articles admit to having volume data but withhold it to join the herd of yay-ASPECTS literature. As a methodological laxity, they offer us only a suboptimal correlate.

Table 4.1: Some Examples of The ASPECTS Woozle.

( CTASI: CTA source images /

CTP: CT perfusion / CBF: CTP blood flow / CBV: CTP blood volume / MTT: CTP mean transit time / CTPM: CTP mismatch / PWI: MRI perfusion )

\begin{tabular}{ll} 
Article & \\
\hline 2000 & Barber et al. \\
2002 & Nabavi et al. \\
$2003 a$ & Hill et al \\
2004 & Molina et al. \\
2005 & Demchuk et al. \\
2005 & Hill \& Buchan (CASES) \\
2005 & Parsons et al. \\
2006 & Dzialowski et al. \\
2006 & Hill et al. \\
2006 & Weir et al. \\
2007 & Aviv et al. \\
&
\end{tabular}

\begin{tabular}{|c|c|}
\hline ASPECTS of ... & Endpoint(s) $^{a}$ \\
\hline CT & $\mathrm{CO}$ \\
\hline CT & $\mathrm{CO}$ \\
\hline CT & TE \\
\hline CT & $\mathrm{CO}$ \\
\hline CT & TE \\
\hline CT & $\mathrm{CO}$ \\
\hline CT, CTASI, CBV, CBF, MTT & FIV as ASPECTS \\
\hline CT & $H T, T E$ \\
\hline CT & $\mathrm{TE}$ \\
\hline CT & $\mathrm{CO}$ \\
\hline CT, CTP & FIV, CO \\
\hline
\end{tabular}

119 Decisions should turn on TE (Nogueira \& Ribó 2019, p. 2615—but despite some single-study TE claims, p. 2616: "we have not yet identified a single imaging-based treatment effect modifier for ET [endovascular thrombectomy]," and cf. Turc et al 2019, online p. 14, and Campbell et al. 2019, pp. 50-1; cf. Wardlaw et al. 2014 for intravenous tPA).

120 Lövblad et al. 1997; Saver et al. 1999; Oppenheim et al. 2000; Thijs et al. 2000; Selim et al. 2002; Albers et al. 2006; Singer et al. 2008; Yoo et al. 2012; Olivot et al. 2013; Marsh et al. 2013; Schaefer et al. 2015; Faigle et al. 2015; Marsh et al. 2016; Streib et al. 2019.

${ }^{121}$ Comparisons favoring DWI lesion volume (Demeestere et al. 2017, pp. 2251-2) and CTP core volume (Demeestere et al. 2018, cf. Harston et al. 2019 and Demeestere et al. 2019) over CT-ASPECTS are not to the point about ASPECTS. For ASPECTS per se: Panni et al. 2019, pp. 1167-8, Tables 2 \& 3, show less overlap for DWI volume than ASPECTS, so that "DWI lesional volume ... emerged as a stronger predictor than DWI ASPECT score"; Honarmand et al. 2013: "ASPECTS > 7 did not correlate with final outcome in patients with successful recanalization." Campbell et al. 2019, p. 53: a $10 \mathrm{~mL}$ increase in core volume by CTP or DWI had similar adverse correlation with outcome as a 5-year increase in age or further 30-minute delay to reperfusion, while "ASPECTS was no longer associated with outcome." Olivé-Gadea et al. 2019 favor ASPECTS, but see notes ${ }^{\mathbf{1 5 3 , 2 7 1}}$ in $\$ \S 5, \mathbf{8}$. For more ASPECTS correlation failures, see $\$ 8$. Using the same software package on the same CT images, volume beat ASPECTS (Brugnara et al. 2020, cf. §6).

${ }^{122}$ Castro Sotos et al. 2009, p. 34, and "its non-transitivity property [is] prone to be neglected by students." Cf.\$2.5. 
Table 4.1 continued from prior page

\begin{tabular}{|c|c|c|c|}
\hline \multicolumn{2}{|l|}{ Article } & \multirow{2}{*}{$\frac{\text { ASPECTS of ... }}{\text { DWI }}$} & \multirow{2}{*}{${\frac{\text { Endpoint }(s)^{a}}{\mathrm{CO}}}^{a}$} \\
\hline 2008 & Kimura et al. & & \\
\hline 2009 & Singer et al. & DWI & $\mathrm{HT}$ \\
\hline 2011 & Nezu et al. & CT, DWI & $\mathrm{HT}, \mathrm{CO}$ \\
\hline 2012 & Sillanpää, et al. & $\mathrm{CT}, \mathrm{CBV}$ & $\mathrm{CO}$ \\
\hline 2013 & Aoki et al. & DWI & $\mathrm{CO}$ \\
\hline 2013 & Honarmand et al. & $\mathrm{CT}, \mathrm{CTP} / \mathrm{PWI}$ & $\mathrm{CO}$ \\
\hline 2014 & Hill et al. & $\mathrm{CT}$ & $\mathrm{CO}$ \\
\hline 2014 & Liebeskind et al. & $\mathrm{CT}, \mathrm{DWI}$ & $\mathrm{CO}$ \\
\hline 2014 & Lum et al. & CT, CTASI, CBV & FIV, CO \\
\hline 2014 & Yoo et al. & $\mathrm{CT}$ & $\mathrm{HT}, \mathrm{CO}$ \\
\hline 2015 & Luvizutto et al. & $\mathrm{CT}$ & $\mathrm{CS}$ \\
\hline 2015 & McTaggart et al. & CT, DWI & $\mathrm{CO}$ \\
\hline $2016 a$ & Goyal et al. (HERMES) & CT (meta-analysis) & TE \\
\hline 2016 & Herweh et al. & CT (e-ASPECTS) & DWI-ASPECTS \\
\hline 2016 & Padroni et al. ${ }^{b}$ & $\mathrm{CBV}, \mathrm{CBF}, \mathrm{MTT}, \mathrm{CTPM}$ & $\mathrm{HT}, \mathrm{FIV}, \mathrm{CO}$ \\
\hline 2017 & Fahmi et al. ${ }^{c}$ & CTASI (manual × 2 + auto) & FIV, $\mathrm{CO}_{\mathrm{NIHSS,mRS}}$ \\
\hline 2017 & Jadhav et al. & $\mathrm{CT}, \mathrm{CTASI}$ & $\mathrm{CO}$ \\
\hline 2017 & Liu et al. & $\mathrm{CT}, \mathrm{CBV}, \mathrm{CBF}, \mathrm{MTT}$ & $\mathrm{HT}$ \\
\hline 2017 & Mokin et al. & $\mathrm{CT}$ & FIV \\
\hline 2017 & Mourand et al. & $\mathrm{CT}$ & $\mathrm{CO}$ \\
\hline 2017 & Naylor et al. & $\mathrm{CT}$ & $\mathrm{CO}$ \\
\hline 2017 & Pfaff et al. & CT (e-ASPECTS) & $\mathrm{CO}$ \\
\hline 2017 & Sallustio et al. & CT, CTASI & $\mathrm{CO}$ \\
\hline 2017 & Tan et al. & $\mathrm{CT}$ & $\mathrm{HT}, \mathrm{CO}$ \\
\hline 2018 & Logan et al. & $\mathrm{CT}$ & $\mathrm{CO}$ \\
\hline 2018 & Nagel et al. & CT (e-ASPECTS) & $\mathrm{CO}$ \\
\hline 2019 & Boisseau et al. & CT, DWI & $\mathrm{HT}$ \\
\hline 2019 & Nishi et al. ${ }^{d}$ & $\mathrm{CT}$ & $\mathrm{CO}$ \\
\hline 2019 & Phan et al. & $\mathrm{CT}$ (meta-analysis) & $\mathrm{CO}$ \\
\hline 2019 & Sundaram et al. & CT, CT (e-ASPECTS), CBV & FIV, CO \\
\hline 2019 & Wollenweber et al. & $\mathrm{CT}$ (presumed, not stated) & $\mathrm{CO}$ \\
\hline 2021 & Voleti et al. & CT, CTASI & CTP core \\
\hline 2021 & Nannoni et al. & $\mathrm{CT}$ & CTP core \\
\hline 2021 & Liebeskind et al. & $\mathrm{CT}, \mathrm{MRI}$ & Growth after $N$ \\
\hline
\end{tabular}

a Defined in text. Not all relations correlated, while perhaps volume would have.

b Perhaps most the aggressively titled: "Cerebral Blood Volume ASPECTS Is the Best Predictor of Clinical Outcome in Acute Ischemic Stroke" (only among ASPECTS).

c Their $9 r_{\mathrm{s}}$ of 3 ratings with 3 measures were only $0.08-0.48$, yet they claim that "Our study supports the usability of automated ASPECTS $\left[r_{s}=0.10-0.24\right]$..." (p. 120).

$d$ They note "the possibility of improving the model because we only included ASPECTS, which is only the simple interpretation of the ischemic core lesion" (p. 2386). 


\section{§5. Kappa Agreement}

ASPECTS was motivated by the old 1/3 MCA rule's “eyeball” technique's unreliability $(\mathbf{\$ 3 . 1 , ~} \mathbf{3}$ ). Without true volume measurements to test its accuracy, ASPECTS's supposed reliability was assessed via just one trait, interrater agreement, which is fundamentally akin to a precision measure (\$2.3). Reliability ideally entails "consistency ... [that goes beyond and] should be clearly distinguished from observer agreement and accuracy," or it might just be agreement "not concerned with the world outside."123 The latter is common, but "when two observers independently agree, ... it is possible ... that they simply share a similar but nonetheless deviant worldview."124 Thus "The degree of agreement among the raters provides no more than an upper bound on the degree of accuracy present in the ratings." 125 It follows that improved observer agreement does not reliably imply improved test or measurement accuracy. ${ }^{\mathbf{1 2 6}}$ Yet ASPECTS was contrasted against the 1/3 MCA rule by the measure of the kappa ( $\boldsymbol{\kappa})$ statistic, "A Coefficient of Agreement for Nominal Scales"127 popularized by psychologist Jacob Cohen.

To explain the concept of $\boldsymbol{\kappa}$, we'll start with a basal version of the most basic discrimination. In a series of trials, raters $\mathbf{A}$ and $\mathbf{B}$ seek the same discrimination and judge it to be + or - (Table 5.1).

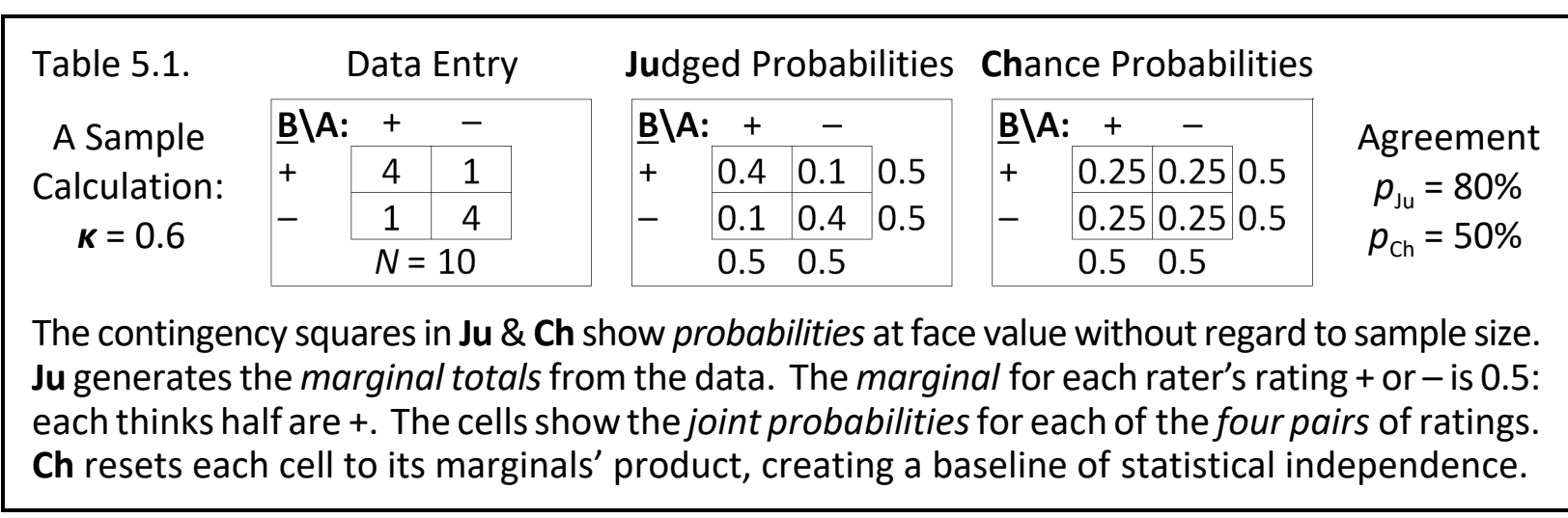

To see how much Judgment really adds to Chance's 50\% agreement for the given marginals, $\boldsymbol{\kappa}$ compares them by the sums, $p_{\mathrm{Ju}}$ and $p_{\mathrm{Ch}}$, of probabilities from only their agreement cells (++,--):

$$
\boldsymbol{\kappa} \equiv \frac{p_{\mathrm{Ju}}-p_{\mathrm{Ch}}}{1-p_{\mathrm{Ch}}}=\frac{(0.4+0.4)-(0.25+0.25)}{1-(0.25+0.25)}=0.6
$$

\footnotetext{
123 quoted respectively: Johnson \& Bolstad 1972, p. 6; Krippendorff 2004a, p. 212.

${ }^{124}$ Bakeman \& Gottman 1997, p. 59. Cf. Krippendorff 2004, p. 213: "In the pursuit of high reliability, validity tends to get lost"; and Bevan 1953, p. 14: "our preoccupation with ... reliability and a tendency to give less consideration to ... validity."

125 Fleiss 1981, p. 212; cf. Sun 2017, p. 151: "Reliability is a necessary but not sufficient condition of validity.” Cf. \$2.3.

126 e.g., El-Tawil et al. 2019, p. 3109: “Agreement on total [CTP-]ASPECT score was substantial to almost perfect” (0.62-0.81 Krippendorff $\boldsymbol{\alpha}$ for CTP variables, p.3108, cf. Supp. pp.17-21). This does not promise "almost perfect” accuracy (not tested). Moreover, "Almost Perfect" is the "clearly arbitrary" "Strength of Agreement" that Landis \& Koch 1977 (p. 165) accord to $\boldsymbol{\kappa} \geq 0.81$ for a "Diagnostic Classification Regarding Multiple Sclerosis" (p.161). Krippendorff 2004a (pp. 241-3) requires $\boldsymbol{\alpha}>0.8$, "a pretty low standard" for research and inadequate "If the outcome ... will affect someone's life ..." (e.g., thrombectomy). Krippendorff 2019 (p. 357) considers the L\&K 1977's "benchmarks ... largely misleading."

127 Cohen 1960, title. Cf. Liebetrau 1983, pp. 32-4; Siegel \& Castellan 1988, pp. 284-91; Zwick 1988.
} 
The raw increase in agreement, $p_{\mathrm{Ju}}-p_{\mathrm{ch}}$, could be stated in $\%$ or in percentage points (\% minus \%). But $\boldsymbol{\kappa}$ is not a $\%$ or $\%$-point increase over $p_{\mathrm{ch}}$, biased by division by $p_{\mathrm{ch}}$. Rather, $\boldsymbol{\kappa}$ divides by $1-p_{\mathrm{ch}}$ to magnify $p_{\mathrm{Ju}}-p_{\mathrm{ch}}$ oppositely from a $\%$ change, showing $p_{\mathrm{Ju}}$ 's fractional gain from $p_{\mathrm{ch}}$ toward 1 .

Perfect agreement inherently matches the raters' splits between + and - . In this circumstance, $\boldsymbol{\kappa}=1$ for any split, as long as there $i s$ a split so that $n_{++}, n_{--}$each $>0$. But if agreement is imperfect, then even for given totals of agreement, $\boldsymbol{\kappa}$ will be modified by the balance between ++ and -- ratings. For example, if Table 5.1's inputs of 4 and 4 change to 7 and 1, giving the same $80 \%$ agreement, $\boldsymbol{\kappa}$ falls to 0.375 . This is valid within the concept of $\boldsymbol{\kappa}$ because the $80 \%$ agreement reflects less value added to the now higher chance agreement. The maximum $\boldsymbol{\kappa}$ (for perfect agreement) is still 1. But in a realistic scenario with enough cases to make perfect agreement essentially impossible, if the underlying balance, or at least the perceived balance as the ratings come in, is uneven, $\boldsymbol{\kappa}$ 's practical ceiling is lowered. Raters should be counseled "not to be discouraged" by these low $\boldsymbol{\kappa} \mathrm{s} .{ }^{128}$

Here are some more $\boldsymbol{\kappa}$ complexities. For a given $p_{\mathrm{Ju}}$, identical splitting $\left(n_{+-}=n_{-+}\right)$maximizes $p_{\mathrm{Ch}}$, so A and B splitting differently raises $\boldsymbol{\kappa}$. With identical rater splits, $\boldsymbol{\kappa}<0$ for any net disagreement, but only if both split evenly can $\boldsymbol{\kappa}$ reach -1 . Yet $\boldsymbol{\kappa}<0$ does not exactly mean "net disagreement," because we can have $p_{\mathrm{Ju}}>1 / 2, \boldsymbol{\kappa}<0$ (e.g., for data $14,10,10,7$ ) and $p_{\mathrm{Ju}}<1 / 2, \boldsymbol{\kappa}>0$ (e.g., for data 18,16,28,25). The behavior is tortuous because of $\boldsymbol{\kappa}$ 's asymmetry and 3 degrees of freedom - as cell proportions (or entries for a given $N$ ) or as the separately variable balance of ratings, balance of agreement, and difference of the raters' split. Over either of these 3D domains, $\boldsymbol{\kappa}$ is a $4^{\text {th }}$-dimensional function.

Plainly, then, $\boldsymbol{\kappa}$ can be hard to master intuitively even in the simplest case of a binary nominal variable (+ vs. - , or apples and oranges) for 2 raters. ${ }^{129}$ The literature attributes the difficulty to the "prevalence," meaning the "observed prevalence." ${ }^{130}$ For $\boldsymbol{\kappa}$, which does not address underlying truth, the prevalence refers to the balance(s) of choices made by the raters - alluded to above in the variables of the (un)evenness of, and of the sameness or difference of, the raters' splittings. The range of $\boldsymbol{\kappa}$ that can be obtained is therefore a function of the true prevalence only to the degree that the raters are sensitive and specific and agree or disagree on the net perceived prevalence.

It's even harder to characterize $\boldsymbol{\kappa}$ 's behavior for 3 raters giving rise to an 8-cell contingency cube, or $n$ raters with $2^{n}$ cells. ${ }^{131}$ As physicians, statistical laymen, we could take $\boldsymbol{\kappa}$ on faith, but should we? Now, what if the exponent's base is $>2$, say, 3 for a ternary nominal variable (apples, oranges, figs), even with just two raters $(9$ cells)? Unless you love mathematics and have a lot of time, you probably are hoping we will not go there-but we must, and beyond, albeit in less detail.

\footnotetext{
128 Bakeman \& Gottman 1997, p. 67.

${ }^{129}$ For controversies and paradoxes of $\boldsymbol{\kappa}$ : Krippendorff vs. Fleiss 1978; Kraemer 1979, Brennan \& Prediger 1981; Zwick 1988; Feinstein \& Cicchetti 1990; Cicchetti \& Feinstein 1990; Brennan \& Silman 1992; Lantz \& Nebenzahl 1996; and next note.

${ }^{130}$ Kraemer 1979, p. 464: "observed prevalence $P$ is generally a biased estimator of true prevalence $P *$ "; cf. Feinstein \& Cicchetti 1990, pp. 543-5. If a prevalence is uneven, using it in Ch might be debatable. Guttman's 1946 (p. 83) "reliability coefficient" $\boldsymbol{\rho}$ ( $S$ of Bennett et al. 1954, p. 307) is $\boldsymbol{\kappa}$ except Ch prevalences are leveled to 1/N (our notation). To compare coefficients, see Krippendorff 2004a (pp. 244-50), — 2004b, Hayes \& - 2007, or van Oest 2019.

131 Light 1971 (pp. 368-70) and Fleiss 1971 “generalized” (p. 378) $\boldsymbol{\kappa}$ or Scott's (1955) $\boldsymbol{\pi}$ to $>2$ raters.
} 
The original use of $\boldsymbol{\kappa}$ for ASPECTS has a grave problem. ASPECTS is not a nominal variable in the first place, but a magnitude variable on its face $(\$ 3)$, however chaotic it is underneath $(\$ 6)$. Dichotomization masks a gradient (\$4). Just as “calling a sheep's tail a leg don’t make it a leg,",132 this sleight of hand does not turn an artificially dichotomized magnitude variable into a nominal one. There still is a wide range of degrees of wrong among its misclassification events, or degrees of

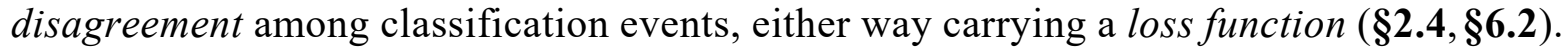

The remedy would be to look behind the dichotomization and weight $\boldsymbol{\kappa}$ by a loss function reflecting the number of steps of disagreement. Cohen's "Weighted Kappa" uses "disagreement weight[s] ... assigned by means of any judgement procedure set up to yield a ratio scale ...."133 The set of weights reflects the shape of the loss function, and "judgment" notes a subjective component in the choice of a loss function (\$2.4). The disagreement weights $\left(v_{i j}\right)$ function in mutual proportions and need not be normalized like probabilities. ${ }^{134}$ In Cohen's example, following the contingency table's diagonals out from the agreement diagonal $\left(v_{i=j}=0\right)$, the weights are successive squares: $1,4,9, \ldots$ (quadratic). ${ }^{135}$ Weighted $\boldsymbol{\kappa}$ is a generalization (different from that for $n$ raters), of which original $\boldsymbol{\kappa}$ is a special case. But because the weighting is user-selected, weighted $\boldsymbol{\kappa}$ s are comparable only with that weighting.

As a measure of agreement between raters of equal status (not a validation against a gold standard), weighted $\boldsymbol{\kappa}$ 's loss function is symmetric, so the weights are too. The issue is the shape of the weighting for successively greater degrees of disagreement on the scale (for us, the scale that was dichotomized). "MedCalc offers two sets of weights, called linear and quadratic." "Th6 "The most common set of weights for the intermediate values is that they are equally spaced [linear]," but "quadratic weighting is common" too. ${ }^{137} \$ 2.4$ suggests that a loss function often is at least somewhat supralinear, but even if for infarct volumes it is just linear (e.g., Fig. 4.1), the next point is medically (and bibliographically) troubling.

The founding article by Barber et al. (\$3.1) reports lower $\boldsymbol{\kappa}$ for " $\leq 1 / 3$ vs $>1 / 3$ MCA" than for "ASPECTS $\leq 7$ vs $>7$," attributing their own knowledge of " $\boldsymbol{k}$ statistics" to "Armitage P, Berry G. Statistical methods in medical research. Oxford: Blackwell Scientific Publications, 1987."138 Even though $\boldsymbol{\kappa}$ (unmodified) is not appropriate for a magnitude variable like volume or ASPECTS, Barber et al. do not mention "weighted kappa."

132 Part of the answer to a riddle possibly used by, but predating (back at least as far as 1825), President Lincoln, e.g., "If you call a sheep's tail a leg, how many legs will a sheep have?"- Quote Investigator 2015.

${ }^{133}$ Cohen 1968, title: "Weighted Kappa: Nominal Scale Agreement with Provision for Scaled Disagreement or Partial Credit." "Nominal" unfortunately crosses over into misnomer, as this work can account for "ratio scaling," at least in steps, of the primary variable (p. 215-also, text's $2^{\text {nd }}$ quote). Cf. Liebetrau 1983, pp. 34-6.

${ }^{134}$ Cohen 1968, pp. 215 (for disagreement weights), 217 (for agreement weights). But many authors normalize them anyway: Fleiss 1981, p. 223; Armitage \& Berry 1994, pp. 445-6; Armitage et al. 2002, p. 701.

${ }^{135}$ Cohen 1968, p. 218. He does not use the phrase "loss function," but the concept is implicit; "cost function" has been misused for it (cf. note ${ }^{17}$ in §2.4), and with this meaning Cohen uses "different costs" for the asymmetric case on p. 219. A problem is that weighted $\boldsymbol{\kappa}$ is sensitive to the weighting (choice of loss function, \$2.4)-Maclure \& Willett 1987.

${ }^{136}$ Medcalc Software, Ostend, Belgium, https://www.medcalc.org/manual/kappa.php, last accessed 2021.12.12.

137 quoted respectively: linear: Armitage \& Berry 1994, p. 446 (and Armitage et al. 2002, p. 701); quadratic: Sim \& Wright 2005 (and used, e.g., by Messé et al. 2007, p. 2081).

138 Barber et al. 2000: statistical results in Table 2, p. 1673; citation in Methods, p. 1672, and References, p. 1674. 
Pexman et al. conclude their Abstract with "ASPECTS ... Clinician agreement is superior to that of the 1/3 MCA rule," supporting this in their TABLE 2 that again cites Armitage \& Berry 1987. They do not mention "weighted kappa" - and highlight this omission by specifying "Balanced $\boldsymbol{\kappa}$,"139 which is not a synonym for weighted $\boldsymbol{\kappa}$ but is instead just a special case of original $\boldsymbol{\kappa}$ wherein the experiment was set up to minimize $\boldsymbol{\kappa}$ 's prevalence-related paradoxes by balancing the observers' positive and negative agreement, ${ }^{140}$ if this really can be done in clinical infarct research.

Had Armitage \& Berry 1987 discussed weighted $\boldsymbol{\kappa}$, then at least we could think these two articles might have used it without taking credit. But Armitage \& Berry 1987 does not discuss $\boldsymbol{\kappa}$ at all. ${ }^{141}$

Pexman et al.'s same citation of Armitage \& Berry 1987 covers for a verbal rating of $\boldsymbol{\kappa}$ in 0.2 steps from excellent to poor, which appears in no edition of Armitage \& Berry but resembles (not exactly quoting) $\boldsymbol{\kappa}$ 's verbal "Strength of Agreement" scale in steps of 0.2 originated by Landis \& Kochan advanced article that is not a how-to for weighted $\boldsymbol{\kappa}$ although it does allude to the concept. ${ }^{142}$

In view of their failure to specify "weighted $\boldsymbol{\kappa}$," their citation for $\boldsymbol{\kappa}$ that does not discuss $\boldsymbol{\kappa}$, and the implausibility of trying to emend their citation to redirect it to the 1994 third edition of Armitage \& Berry (or in Pexman et al.'s case to Landis \& Koch), we cannot credit Barber et al. and Pexman et al. with using weighted $\boldsymbol{\kappa}$, and they probably in fact did not use weighted $\boldsymbol{\kappa}$.

In ASPECTS-agreement research, unweighted $\boldsymbol{\kappa}$ is wrong ${ }^{143}$ because ASPECTS is a $>2$-step scale and its wide range of disagreements (\$6) promises clinically significant errors. (Even weighted $\boldsymbol{\kappa}$ does not suffice to compare ASPECTS to $1 / 3$ MCA because volume is a continuous variable.$^{144}$ ) We want to know how badly patients are misclassified. High dispersion causes threshold-crossing errors to affect some patients who are far from the threshold, deserving distinctly higher $\boldsymbol{\kappa}$ weights.

ASPECTS's problems with agreement so far are: (1) agreement does not validly promise accuracy; (2) agreement without accuracy is pointless or worse; (3) ASPECTS has not had accuracy verified; (4) $\boldsymbol{\kappa}$ 's mathematical coherence does not make it perspicuous or suitable; (5) $\boldsymbol{\kappa}$ was often misused; (6) the comparison was made to a straw man (\$3.1).

139 Pexman et al. 2001, p. 1540

${ }^{140}$ Kraemer 1979, pp. 464-5, 469-70); Feinstein \& Cicchetti 1990, p. 545; Cicchetti \& Feinstein 1990, pp. 556-7; Lantz \& Nebenzahl 1996, p. 433: "balanced kappa."

${ }^{141}$ Armitage \& Berry 1987. Kappa and weighted $\boldsymbol{\kappa}$ make their first appearance in Armitage \& Berry in 1994, pp. 443-7, but this is not what either article cites.

142 Landis \& Koch 1977, p. 165 for the verbal rating, pp. 162 passim for allusions to weighting. Laudatory verbal ratings like "Substantial" are conceived for exploratory scientific interest, not for applied adequacy in engineering or patient uses, and even in research might better be thought of as a vocabulary for propaganda — cf. page 29 note $^{\mathbf{2 6}}$.

143 "Unweighted kappa ... is inappropriate for ordinal scales"—Sim \& Wright 2005, p. 259; cf. Maclure \& Willett 1987. Coutts et al. 2004, p. 2, Butcher et al. 2008, p. 76, Singer et al. 2009, p. 2745, Alexander et al. 2012, p. 739, and de Margerie-Mellon et al. 2013, p. 3565, use weighted $\boldsymbol{\kappa}$. For an interesting look at agreement by region categorically, where unweighted $\boldsymbol{\kappa}$ is appropriate, see Gupta et al. 2012, p. 1048: caudate and insula highest, IC by far lowest.

${ }^{144}$ Maclure \& Willett 1987, p. 162: "the most obvious misuse of Kappa —its application to continuous data ... often grouped, for the convenience of the investigator, into categories of arbitrary number and size." There are other agreement statistics besides $\boldsymbol{\kappa}$, such as the intraclass correlation coefficient (Maclure \& Willett 1987, Finlayson et al. 2013), and Krippendorff's $\alpha$ (“ $\alpha$-Agreement," Krippendorff 2004, pp. 221-56). 
Admissions listed in an article that seems overall to advocate, ironically, for ASPECTS effectively retract its initial motivation: ${ }^{145}$

Further studies have suggested reasonable agreement among experienced readers for detecting $>1 / 3$ MCA territory infarction,

ECASS-3 ... central review ... identified CT scan protocol violations in only $2.1 \%(n=17)$, implying that investigators were very successful in recognising evidence of large infarction [ $\left.{ }^{146}\right]$, Mak et al. $\left[{ }^{147}\right]$ found a higher reliability with the one-third rule $(\boldsymbol{\kappa}=0.49)$ compared with dichotomized ASPECTS $(\boldsymbol{\kappa}=0.34)$,

Current data do not consistently prove higher reliability of ASPECTS [vs. $1 / 3$ MCA rule].

To the $1 / 3$ MCA rule's reasonable agreement and higher reliability and the other remarks above, add these other voices:

RESULTS Systematic review.... ASPECTS ... weighted kappas varied between 0.21 and 0.75 .... DISCUSSION ... our own clinical practice ... did not show sufficient agreement between clinicians for ASPECTS to be reliably used for treatment decisions. ${ }^{148}$

... the well-established interreader variability inher[en]t in ASPECT scoring. ${ }^{149}$

ASPECTS may not allow reliable, appropriate patient selection for endovascular therapy. ${ }^{150}$

Agreement between clinicians assessing DWI-ASPECTS ... may not be sufficient to make repeatable clinical decisions in mechanical thrombectomy. ${ }^{151}$

[O]nly moderate to substantial agreement was recorded between consensus of the two neuroradiologists and the neuroradiology fellow, with slight to moderate agreement between consensus of the two neuroradiologists and the resident. ${ }^{152}$

[E]ven between experts, ASPECTS presents a poor interrater reliability. ${ }^{153}$

Finally, coming up, §6.2's ACCESS data further nullify ASPECTS's original raison d'être, showing instead that ASPECTS is a crude and confused descriptor of infarct extent.

145 Puetz et al. 2009, pp. 356-7 for the following four quotes from this article on the Calgary group’s own update (\$3.2).

146 attributed to Hacke et al. 2008; Michel et al. 2012 make the same attribution for the same "2.1\%." Hacke et al. 2008 do not say this, yet there may be something to it because Schellinger et al. 2003, p. 576, with W. Hacke as co-author, say, of the original claim that "interobserver reliability of ASPECTS was higher than that of the one third MCA rule," that "other preliminary data contradict these findings (W. Hacke, oral personal communication, 2002)."

147 Mak et al. 2003, esp. Table 1, p. 1195.

148 Farzin et al. 2016, pp. 252,254.

${ }^{149}$ Maegerlein et al. 2019, p. 147 (stated, as general knowledge, as a premise for one of their methodological limitations).

150 Jadhav \& Wechsler 2016, p. 242.

151 Fahed at al., 2018a, p. 223

152 Kobkitsuksakul et al. 2018, p. 107 ("only moderate to substantial" with the fellow, not stated between the neuroradiologists).

153 Olivé-Gadea 2019, p. 198 (advocating for automated software, §10 — but that would be just one black-box rater); cf. the mediocre overall and regional $\boldsymbol{\kappa}$ s in Nicholson et al. 2020, even leniently by L\&K (above) mostly only "Moderate." 
Page 34 ASPECTS, The Mismeasure of Stroke: A Metrological Investigation

\section{§6. How Not to Measure Volume: ASPECTS}

\section{$\S 6.1$ The Real Anatomy of ASPECTS}

The biggest red flag that ASPECTS waves is its bizarre scale for measuring infarct volume. The ASPECTS Ruler (Fig. 6.1) is a 1-dimensional metaphor for measuring a 3-dimensional volume, with segment lengths proportional to ASPECTS region volumes from anatomy research (Table 6.1). Heuristically it imagines the regions to be involved successively, starting from the small ones. With the official preference to count only the PLIC as the IC region (\$3.2), the ALIC is partitioned.

\begin{tabular}{lll}
\hline Table 6.1. & Caudate Head + 1/2 ALIC & $\approx 4.5 \mathrm{~cm}^{3}-^{154}$ \\
ASPECTS & Lentiform Nucleus + 1/2 ALIC & $\approx 6.5 \mathrm{~cm}^{3}-{ }^{155}$ \\
Anatomy. & Post Limb Internal Capsule & $\approx 2.5 \mathrm{~cm}^{3}-{ }^{156}$ \\
& Insula + ExtremeCap-Clau-ExtCap & $\approx 7.5 \mathrm{~cm}^{3}-{ }^{157}$ \\
&
\end{tabular}

If just the four small regions are involved, then at ASPECTS 6 this $\leq 21 \mathrm{~cm}^{3}$ is $\leq 7.4 \%$ of the average $284.4 \mathrm{~cm}^{3}$ MCA territory $(\$ 4){ }^{158}$ A CT in $\$ 7$ suggests a volume of $26 \mathrm{~cm}^{3}, 8.6 \%$ of its estimated MCA territory. With the biases of varied sizes and any part scoring (\$3.2), $<9 \%$ of MCA volume can reach $40 \%$ of the ASPECTS scale. (The cortex-only interpretation of ASPECTS, refuted in \$3.2, would somewhat reduce the M-region volume excess at the price of ignoring most of the white matter.) An infarct of the insula, caudate, and lentiform regions extending slightly into M1 and M2, at $\ll 25 \%$ but reaching ASPECTS 5, could unjustly deny the patient a salutary intervention $(\$ 7, \S 9)$.

The ASPECTS Ruler displays an unprecedented scale of bias and disproportionality.

154 Ertekin et al. 2013, p. 305, Table 1: Caudate 3.6, 0.9 for half of ALIC in Goghari et al. 2011, p. 625, Fig. 3.

155 Ertekin et al. 2013, p. 305, Table 1: Lentiform 5.6, + 0.9 for half of ALIC in Goghari et al. 2011, p. 625, Fig. 3.

156 Goghari et al. 2011, PLIC estimated from p. 625, Fig. 4. As the smallest and most controversially defined region (§3.2), the IC (PLIC) has the lowest regional interrater agreement in ASPECTS (Hill et al. 2003b; Finlayson et al. 2013).

157 Insula: $5.3 \mathrm{~cm}^{3}$ is estimated from Cohen et al. 2010: p. 87, fig. 4. Extreme capsule: $1 \mathrm{cc}$ is estimated from data in Makris \& Pandya 2009, p. 349, Table 1, adjusted by data on p. 346, Fig. 2d. Claustrum ("historically defined as the thin strip of gray matter interposed between the striatum and the insular cortex" -Mathur 2014, p. 2): $1 \mathrm{~cm}^{3}$ is estimated from Lemaitre et al. 2012, p. 617.e5: "average cortical volume was $458 \pm 52 \mathrm{~cm}^{3}$," and Baizer et al. 2014, p. 4: claustrum is $0.45 \%$ of isocortex (which is most of the foregoing cortical volume, then dividing by 2 for one hemisphere). External capsule: $0.2 \mathrm{cc}$ is estimated from data in Makris \& Pandya 2009, p. 350, Table 3.

158 van der Zwan et al. 1993, p. 1955. Variation is expected among people ( $\pm \approx 14 \% 2 S D$, Erteken et al., op. cit.) and studies: Ros Mendoza et al. 2009 add $0.7 \mathrm{cc}$ to the caudate + lentiform, and Wang et al. 2012 add $0.8 \mathrm{cc}$ to the lentiform (no caudate figure), bringing the total small-region volume to $21.7-21.8 \mathrm{~cm}^{3}$ and its fraction of total MCA territory volume to $7.7 \%$. 
Only in 2006 was it first pointed out in print that "The ASPECTS template is weighted in favor of the striatocapsular region." 159 Other writers have subsequently pointed out that:

Equal weighting is given to smaller structures (such as the internal capsule, basal ganglia, and caudate nucleus) as is given to larger cortical areas; ${ }^{160}$

[T] he anatomic regions covered by ASPECTS are not equally distributed across the middle cerebral artery (MCA) territory. There are 3 score items allotted to subcortical regions ..., whereas 7 score items represent cortical brain areas ... across the entire MCA territory; ${ }^{161}$ $[T]$ he template is unequally weighted ... correlation with lesion volume depends on ... location; ${ }^{162}$ Individual ASPECTS regions are not equally weighted, and correlation with stroke lesion volume is low in subcortical regions; ${ }^{163}$

[S]ome regions ... are much smaller than others, yet they are equally weighted in the total score; thus two patients with the same ASPECTS score may not have the same extent of EIC. ${ }^{164}$

It is not clear whether even these cited authors realize how overweighted the four small regions are. If regions totaling $\approx 8 \%$ of the MCA volume get $40 \%$ of the ASPECTS score, they are overweighted, compared to the M regions, by a factor of (92\%/6 regions)/(8\%/4 regions), between 7 and $8 .{ }^{165}$ The founding articles do not claim overweighting as a virtue. Later articles (\$8) rationalize it as "functional weighting" but offer no analysis rationalizing ASPECTS's particular pattern of it. ${ }^{166}$ In looks by ASPECTS regions, outcome usually correlates mainly with the M (convexity) regions. ${ }^{167}$

While the variety of region sizes challenges ASPECTS's classification as a scale (\$2.1), a valid volume surrogate must be not just an ordinal but a ratio scale. If it has categorical steps, it still must reasonably approximate a ratio scale. From our Ruler heuristic of starting with the small regions, a model departing more realistically toward a random order might be stochastically interval, each increment being of about the same size on the average. This model is worth a closer look.

${ }^{159}$ Phan et al. 2006: title; p. 481: "to our knowledge, involvement of the striatocapsular region by infarct has not been shown to be more likely to lead to parenchymal hemorrhage following thrombolysis than involvement of the $\mathrm{M}$ or non-M cortical regions." Loh et al. 2009 (50 patients) found worse disability and more parenchymal hematomas in ganglionic than cortical infarcts, while Spiotta et al. 2015 (149 patients) found similar rates of disability and hemorrhage in these pure types.

160 Puetz et al. 2009, p. 355, and Menon et al. 2011, p. 408. While this imbalance may seem obviously bad, the authors seem to be advocating for it, without justifying it. Correction: the caudate nucleus is part of the basal ganglia.

161 Schröder et al. 2014, p. 3584. Following "However, there are limitations to ASPECTS," the tone is adverse.

162 Schröder \& Thomalla 2017, pp. 1,4. Embedded in other criticisms, the tone is adverse.

163 Schröder et al. 2018, p. 2. Following "However, there are limitations to ASPECTS," the tone is adverse.

164 Wilson et al. 2018, p. 143. Following "An additional criticism of ASPECTS is that ...," the tone is adverse.

165 A ratio of \%/region quotients for individual or aggregate regions should be 1:1. But ASPECTS's 92\%:8\% volume ratio for its $6: 4$ regions gives it a \%/region:\%/region ratio of 72/3:1, effectively overweighting its 4 small regions $\times 72 / 3$.

166 Armitage et al. 2002, p. 456: "some analysis is required to determine which variables might be sensibly combined."

167 a Alexander et al. 2012, ${ }^{b}$ Phan et al. 2013, ${ }^{c}$ Rangaraju et al. 2015, ${ }^{d}$ Beare et al. 2015, ${ }^{e}$ Khan et al. 2017, ${ }^{f}$ Fukuda et al. 2017, ${ }^{g}$ Sheth et al. 2018, ${ }^{h}$ Rosso et al. 2019, ${ }^{i}$ Haranhalli et al. 2020, ${ }^{j}$ Seyedsaadat et al. 2020, ${ }^{k}$ Seyedsaadat et al. 2021 : best predicted by (side $=\mathrm{R}, \mathrm{L}, \mathrm{P}[\mathrm{Pooled}])$ Ins. $\left(\mathrm{P}^{i}\right)$, Caud. $\left(\mathrm{L}^{h} \mathrm{P}^{k}\right)$, Lenti. $\left(\mathrm{L}^{j} \mathrm{P}^{b}\right), \mathrm{M} 1\left(\mathrm{R}^{j} \mathrm{~L}^{j} \mathrm{P}^{a}\right), \mathrm{M} 2\left(\mathrm{R}^{e} \mathrm{~L}^{j} \mathrm{P}^{d i}\right), \mathrm{M}^{2}\left(\mathrm{R}^{e h} \mathrm{~L}^{e g}\right)$, M4 $\left(\mathrm{R}^{g} \mathrm{~L}^{c j} \mathrm{P}^{a f k}\right), \mathrm{M} 5\left(\mathrm{R}^{j} \mathrm{~L}^{\text {ghhj }} \mathrm{P}^{a d i}\right), \mathrm{M} 6\left(\mathrm{R}^{c e g h j} \mathrm{~L}^{\text {egj }} \mathrm{P}^{b f}\right)$ - often unclear whether controlled for confounding by volume (\$8). 
Table 6.2. Frequency Distribution of ASPECTS Region Involvements: Balance of Small and Large Regional Involvement.

\begin{tabular}{|c|c|c|c|c|c|c|c|c|c|c|}
\hline & \multicolumn{3}{|c|}{$\begin{array}{r}\text { "Frequency of ischemic } \\
\text { changes, \%" }(\mathrm{CT})^{168}\end{array}$} & \multicolumn{3}{|c|}{$\begin{array}{l}\text { "Distribution of ... } \\
\text { DWI ASPECTS ..." }\end{array}$} & \multicolumn{3}{|c|}{$\begin{array}{l}\text { "24-h [CT-]ASPECTS } \\
\text { subcomponent" }\end{array}$} & \multirow{2}{*}{$\begin{array}{l}\text { Mean of studies, } \\
n \text {-weighted }\end{array}$} \\
\hline & $n=607$ & $\sum$ & Balance & $n=213$ & $\sum$ & Balance & $n=342$ & $\sum$ & Balance & \\
\hline Caudate & $19.6 \%$ & \multirow{4}{*}{83.8} & \multirow{4}{*}{$43.7 \%$} & $57.5 \%$ & \multirow{4}{*}{174.0} & \multirow{4}{*}{$38.1 \%$} & $54.2 \%$ & \multirow{4}{*}{200.1} & \multirow{4}{*}{$57.5 \%$} & \multirow{4}{*}{$46.7 \%$} \\
\hline Lentiform & $31.0 \%$ & & & $32.1 \%$ & & & $70.5 \%$ & & & \\
\hline Internal Capsule & $0.7 \%$ & & & $59.4 \%$ & & & $18.8 \%$ & & & \\
\hline Insula & $32.5 \%$ & & & $25 \%$ & & & $56.6 \%$ & & & \\
\hline M1 & $19.3 \%$ & \multirow{6}{*}{107.9} & \multirow{6}{*}{$56.3 \%$} & $55.7 \%$ & \multirow{6}{*}{282.9} & \multirow{6}{*}{$61.9 \%$} & $18.8 \%$ & & & \multirow{6}{*}{$53.3 \%$} \\
\hline $\mathrm{M} 2$ & $27.2 \%$ & & & $33 \%$ & & & $38.0 \%$ & & & \\
\hline M3 & $9.2 \%$ & & & $51.4 \%$ & & & $20.2 \%$ & & & \\
\hline M4 & $13.7 \%$ & & & $55.7 \%$ & & & $22.2 \%$ & 147.8 & $42.5 \%$ & \\
\hline M5 & $27.9 \%$ & & & $23.1 \%$ & & & $30.7 \%$ & & & \\
\hline M6 & $10.6 \%$ & & & $64 \%$ & & & $17.9 \%$ & & & \\
\hline & & \multicolumn{2}{|c|}{ 191.7\%-points } & & \multicolumn{2}{|c|}{$456.9 \%$-points } & \multicolumn{3}{|c|}{$347.9 \%$-points } & \\
\hline
\end{tabular}

Method of analysis: The individual regional \%-age involvements are listed. They are summed, first separately for the four small and six large regions, and then all are totaled together. The Balances between the initial sums are stated as \%-ages adding up to $100 \%$. Across these Balances an $n$-weighted average is taken. Coincidentally, the raw $\left(\sum\right) \%$-involvement-weighting gives $47.0 \%: 53.0 \%$.

168 Phan et al. 2013, p. 562, Table 2 ( $n=607)$.

169 Rangaraju et al. 2015, p. 1789, Table $2(n=213)$. The IC and insula involvements are anomalously high and low, respectively, as if they might have been switched, but this poses no problem for our calculation.

${ }^{170}$ Sheth et al. 2018, p. 537, measured from Fig. 1 ( $n=342$ ). Compared to the only three \%-ages stated, Caudate $52 \%$, Lentiform $70 \%$, Insula $55 \%$, the measurements appear to be some 1-4\% greater, but this error is small and all 10 measurements are used together for proportional consistency. 
Table 6.2 displays three studies of the frequencies by which ASPECTS regions are involved. If regions were involved in proportion to their volumes, the ratio of large- to small-region involvement would be about $92 \%: 8 \%$, as derived above. But if their frequencies of involvement go numerically by nominal region irrespective of their volumes, the ratio of large- to small-region involvement will be $60 \%: 40 \%$. As varied as their aggregate frequency involvements are, cautioning that there can be further variation outside this range in other clinical settings or study groups, all three studies nevertheless come much closer to the latter than to the former. The first two come very close, while the last reverses the ratio toward the small regions. Beware, though, of a mathematical illusion: this manifest near-balance in frequency of region involvement has nothing whatsoever to do with, and does not in any way justify, ASPECTS's biased regional weighting for individual patientsmore on this instead in the second paragraph below.

The frequency balance, achieving a fair approximation to region-by-region impartiality, offers some empirical support for a tentative notion, and only a stochastic one (incorporating chance) at that, of ASPECTS as an interval or ratio scale. When just one or two regions are involved, as is most usual in published series, it or they are numerically (not by volume) approximately proportionally likely to be small or large. Additional regions probably follow similar proportions, so that on average the ASPECTS Ruler progresses more evenly than drawn. Still uncertain are the potentially diverse biases of the 10 regions' categorical scoring, which (beyond canceling or reinforcing under- or overestimation in an individual) may differ on average among the regions. But the significance of this stochastically interval behavior is mainly heuristic, to support §6.2's demonstrative use of the standard deviation.

In response to the empirical regional outcome weightings discussed above, Sheth et al. ${ }^{171}$ tried to counterbalance ASPECTS as a "weighted ASPECTS (wASPECTS)" based on parameter estimates. Page 35 's note ${ }^{167}$ shows how variable these parameters can be. Indeed wASPECTS improved outcome estimation on the right only, while worsening it by almost as much on the left - suggesting a chance result. Trying to salvage ASPECTS by weighting its incorrect primary weighting calls to mind the convolutions of the Ptolemaic geocentric and Copernican circular-orbit cosmologies, calculating iterated epicycles in order to rationalize astronomical observations within these incorrect theories. ${ }^{172}$

Epicycle mathematics did work, perhaps misleadingly well. But ASPECTS's faulty theory causes uncorrectable noise, as $\$ \S 6.2-6.3$ show it to be inherently scattershot. ASPECTS is imprecise overall, so in patients it is often inaccurate (\$2.3), often by multiple steps. \$6.2 examines interrater variation among 259 raters of $32 \mathrm{CT}$ scans, while $\$ 6.3$ examines variations among infarcts themselves in how chaotically (one to many and many to one) their volumes map to DWI-ASPECTS. Such imprecision and inaccuracies are to be expected from ASPECTS's coarse steps, heterogeneous region sizes, M-region vagueness, and user inconsistencies. That ASPECTS correlates $(\mathbf{\$ 2 . 5 ,}, \mathbf{8})$ with volume, and that for any volume threshold it classifies a majority of infarcts correctly, might be clinically useful only if direct volume measurement did not exist and nothing better than ASPECTS were available. But volume is directly measurable and something better than ASPECTS is readily available in §7.

\footnotetext{
171 Sheth et al. 2018, pp. 535, 537-41.

172 Neugebauer 1957/1969, pp. 123, 191-207; Hanson 1960.
} 


\section{$\S 6.2$ A Further Analysis of Some CT-ASPECTS Data}

CT's deficiencies are that (1) the earliest infarcts, sometimes up to 2-3 hours, are truly invisible, (2) some visible ones are too subtle for many readers to recognize, (3) cortical sulcation and pixel noise can simulate hypodensities that are mistakable prospectively or retrospectively for early infarcts, (4) artifacts (motion, bone, hardware) can hide or produce hypodensities simulating acute infarcts, and (5) acute infarct hypodensity can resemble (false negative) or be resembled by (false positive) chronic ischemic hypodensity. These deficiencies affect the detection of any infarct at all and the detection of parts of infarcts for determination of infarct extent.

When an acute infarct is discernible, ASPECTS masks volume information by adding noise through its scale irregularities. Noise is any error, random or systematic, that muddles the message, or "A disturbance that tends to, or does, interfere with the proper operation of a device or a system."173 The noise of ASPECTS invites measurement of its informational harm. Fortunately the large ACCESS dataset, made available through the courtesy of JM Wardlaw and F Chappell, lets us begin to look past CT's deficiencies to the deleterious effect of ASPECTS on denoting infarct extent. Fig. 6.2 replots these authors' Figure 3 with the scans relabeled, reordered, and encoded for the ratings by their "reference standard neuroradiologist"(rsNR) as the ostensible truth, even though, admittedly, "1 expert's CT scan readings are not a perfect 'gold standard'.,"174

Fig. 6.2 includes two familiar scales, < vs. $>1 / 3$ of the MCA territory (\$4) and ASPECTS (\$3), while the IST-3 Scale ${ }^{175}$ needs an introduction. It is an omnibus index ${ }^{176}$ of three infarct subscales: "Site and size," "Swelling," and "Hemorrhagic transformation." While the latter two are ordinal, the first, at least at its originally finest MCA detail as shown, is itself an omnibus index of sites and sizes, with variously serial (ordinal superset-subset) or parallel (e.g., anterior vs. posterior MCA territory) relations between its consecutively listed, but not necessarily uniquely consecutive, values. This complexity makes it a hybrid ordinal and nominal scale (\$2.1). The IST-3 scale nevertheless serves as a control for CT-ASPECTS because it has nearly as many values which, likewise, must be distinguished by discerning infarct presence and extent.

Since we are investigating ASPECTS not merely as a detection tool (a check list's job) but as a volume scale for observed acute infarcts, the negative ratings (same for all scales) are set apart as Fig. 6.2's bottom rank to favor comparison among the positive ratings above. Then assuming the rsNR's reliability, the 32 scans are distinguished as negatives (lettered) and positives (numbered). The true positives of the 22 numbered rsNR-positive scans are analyzed further in Fig. 6.3.

\footnotetext{
173 Weik 1996, p. 628.

174 ACCESS citations: Wardlaw et al. 2010 (1 $1^{\text {st }}$ quote: passim; Figure 3: p. 6); Wardlaw et al. 2007 (2 $2^{\text {nd }}$ quote, p. 1251).

175 Wardlaw \& Sellar 1994. In the IST-3 study, it is condensed from its full form for all territories and with the 8 categories of MCA abnormality labeled in Fig. 6.2, down to an ordinal "small," "medium," "large," and "very large" (IST-3 Collaborative Group 2015, p.488), or to just "Small or medium" and "Large or very large" (Berge et al. 2016, p. 1030).

176 usage: Cicchetti \& Feinstein 1990, p. 551 (too narrowly: "a single expression that summarizes the results of a $2 \times 2$ table”); Feinstein 2002, p. 418; Vaidyanathan et al. 2009, passim, most clearly on p. 83: "an omnibus index based on multiple phenotypic indicators." How to weight its components can be an issue. Cf. “intelligence as a single quantity," §2.2.
} 
Fig. 6.2. Ratings of 32 CT Scans by 259 Raters Using $1 / 3$ of the Supposed MCA Territory, the Descriptive IST-3 Scale, and ASPECTS The circles' areas are proportional to the counts, which include the ratings by the Reference Standard Neuroradiologist (rsNR, $\star$ ).

\section{$1 / 3$ of MCA Territory \\ involvement $>1 / 3 \cdot \cdot \cdot \cdot \cdot \circ \circ 9 \cdot \cdot(A) \circ 0 \circ \delta$.

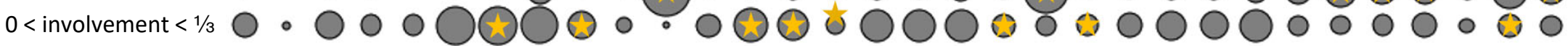

IST-3 Scale ( $N=8$ values)

Whole territory of MCA

BG + Whole cortical MCA .

Whole cortical MCA

Posterior cortical MCA

Anterior cortical MCA .

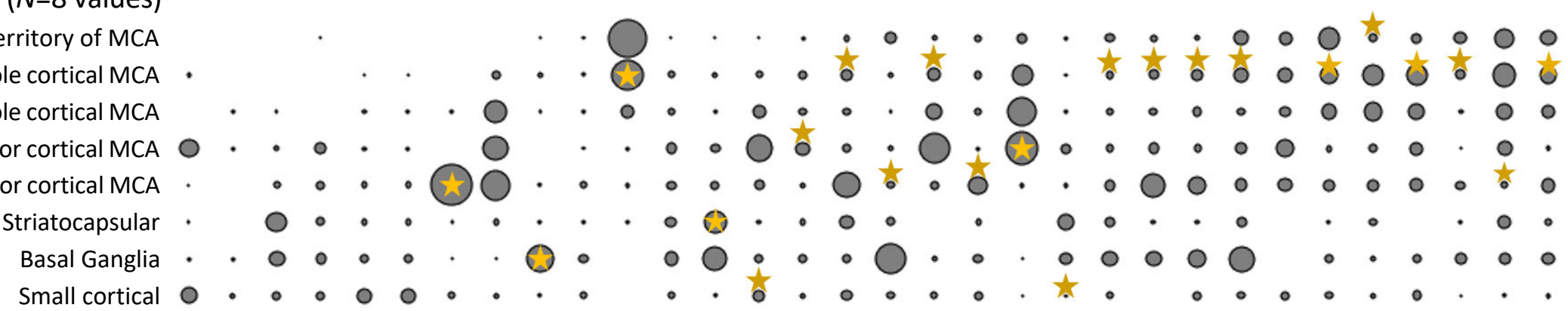

ASPECTS ( $N=10$ values)

\begin{tabular}{|l|}
\hline Figure after \\
Ward la w \\
et al. 2010, \\
p.6, Fig. 3, \\
redra wn \\
in Excel ${ }^{\circledR}$ \\
from data. \\
\hline
\end{tabular}

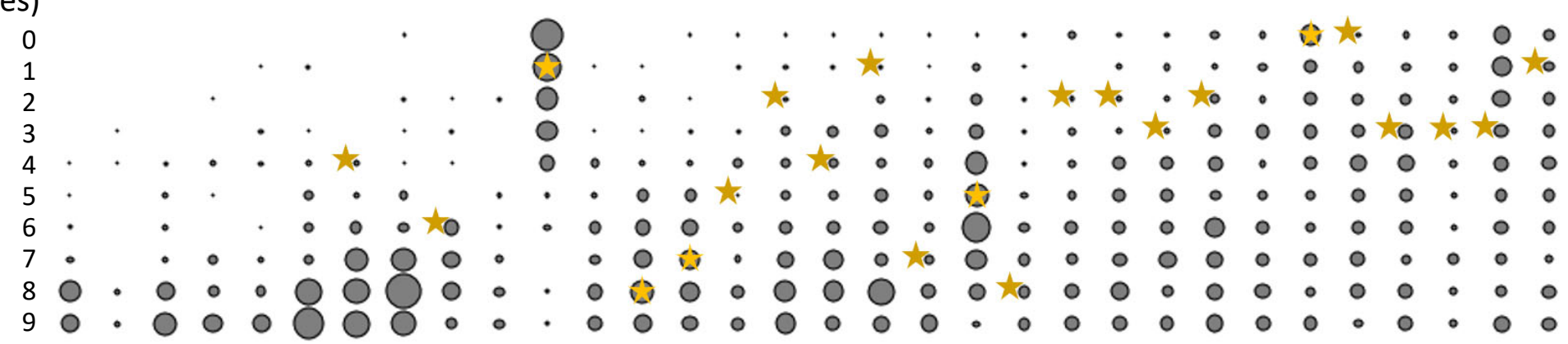

ALL THREE SCALES

No acute infarct seen 10

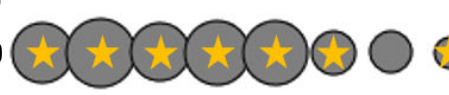

$\begin{array}{lllllllllllllllllllllllllllllllllllllll}32 & \text { SCANS RE-LABELED: A } & \text { B } & \text { C } & \text { D } & \text { E } & \text { F } & 1 & \text { G } & 2 & \text { H } & 3 & \text { I } & 4 & 5 & 6 & 7 & 8 & 9 & 10 & 11 & 12 & 13 & 14 & 15 & 16 & \text { J } & 17 & 18 & 19 & 20 & 21 & 22\end{array}$ Scans are listed $L \rightarrow R$ by decreasing entropy of their abnormal ASPECTS (9-0) distributions. Most of the scans (26) were of good quality, and only one was poor. Numbered scans ostensibly show acute infarcts, per the rsNR, and are re-examined in Fig. 6.3. Abnormal ratings of lettered scans would then be false positives. 
Fig. 6.2's "Bubble Chart" (Microsoft Excel ${ }^{\circledR}$ ) encodes the data as circle areas in a proportional area chart. Its spatial efficiency compares 259 raters $\times 32$ scans $\times 3$ scales $=24,864$ data points. For any of Fig. 6.2's three scales including the bottom rank of true and false negatives, each scan's circles total the same area. For a given scan and scale, the individual areas are proportional to, and compare, the ratings' probabilities (as observed relative frequencies). The original authors pointed out that "For the 1/3 MCA and IST-3 scores the bubbles are more clustered than for the ASPECTS ...."177_ showing better interobserver agreement $(\mathbf{\$ 3}, \mathbf{\S 5})$ for IST-3 than for ASPECTS. Dichotomizing would artificially cluster ASPECTS - artifactually differently for its different arbitrary dichotomizations. From the chart one might guess that its dichotomous clustering (asymmetry of circle sizes for a scan) would naturally increase away from a minimum (at $8 / / 7$ or $7 / / 6$, to be quantified below). Clustering (decisiveness or informativeness among each scale's positives, with or without an ASPECTS dichotomy) is amenable to precise and useful mathematical expression, as developed below.

Only the true positives exhibit the geometric distortions caused by ASPECTS in stroke. False positives (whose false scores are clinically less dangerous) are a flaw of CT, not ASPECTS. For the 22 rsNR-positive scans, Fig. 6.3 displays the conditional probabilities, given a positive rating. The bars for each scan's $n$ true-positive scores are renormalized to equal total height for each scan.

Fig. 6.3 reverts from proportional area circles to bar heights (both invented by William Playfair, in 1801 and 1786, respectively ${ }^{178}$ ), (1) because with fewer data we don't need such spatial efficiency, (2) for the greater precision with which the graphic programs seem to draw bars, ${ }^{179}$ and (3) to better reflect the linearity of the underlying measure (counts of ratings) and averts any criticism that, with circle areas, the competing perceptual salience of diameter vs. area ${ }^{180}$ might tend to make the ratings' dispersions look more evenly spread (even less useful) than they really are.

Probabilities, not counts, are displayed because (1) as $n$ varies, probabilities remain comparable, and (2) probability offers an established method to compare, between scans or scales (for any level of discrete scale, \$2.1), the clustering or, inversely stated, the evenness of dispersion of the ratings. We will proceed to explain this method of comparison.

177 Wardlaw et al. 2010, p. 5. The balance of MCA-fraction scores varies with the fraction, to be discussed below.

178 The Statistical Breviary (1801) and The Commercial and Political Atlas (1786) as cited respectively by Spence 2005, p. 354, FIGURE 1, and by Spence 2006, p. 2432, 2.3.3 and Figure 16.

179 as I compare measurements of the circles and bars to the counts. Concerns about graphical imprecision and inaccuracy caused lingering resistance to Playfair's inventions-Spence \& Wainer 2005, pp. 24-5.

${ }^{180}$ Attention to perceptual salience is cued by Piaget's observations on conservation of quantities: children aged 5-6 years may vacillate between the height or $\approx$ height $\times$ width of a liquid column to decide whether the quantities are the same (Piaget 1941/1952, pp.13-17; Ginsburg \& Opper 1988, p. 152). Reminiscent of that phenomenon, but in adults, length is easier than area to judge and compare, as if, again, the implied multiplication slows or confuses the process, or (perhaps for a related reason) an object's diameter has its own salience that competes with its area. This phenomenon favors bars over circles (or other areas) for many graphical uses. The tendency to underestimate a ratio of circle areas varies in degree among contexts, persons, and occasions (with exponents of 0.80-0.91 having been claimed too narrowly) so that no systematic correction is possible, except reverting to bars. However, since the purpose of charts is to make comparisons, the choice of any type of graph symbol depends on the nature of the data and the communicative purpose. See Flannery 1970; MacDonald-Ross 1977, pp. 369-75; Griffin 2017, p. 47. 
Figure 6.3. True-Positive CT-ASPECTS (9-0): 22 Scans Positive by rsNR $(\star)+52-245$ Other Raters

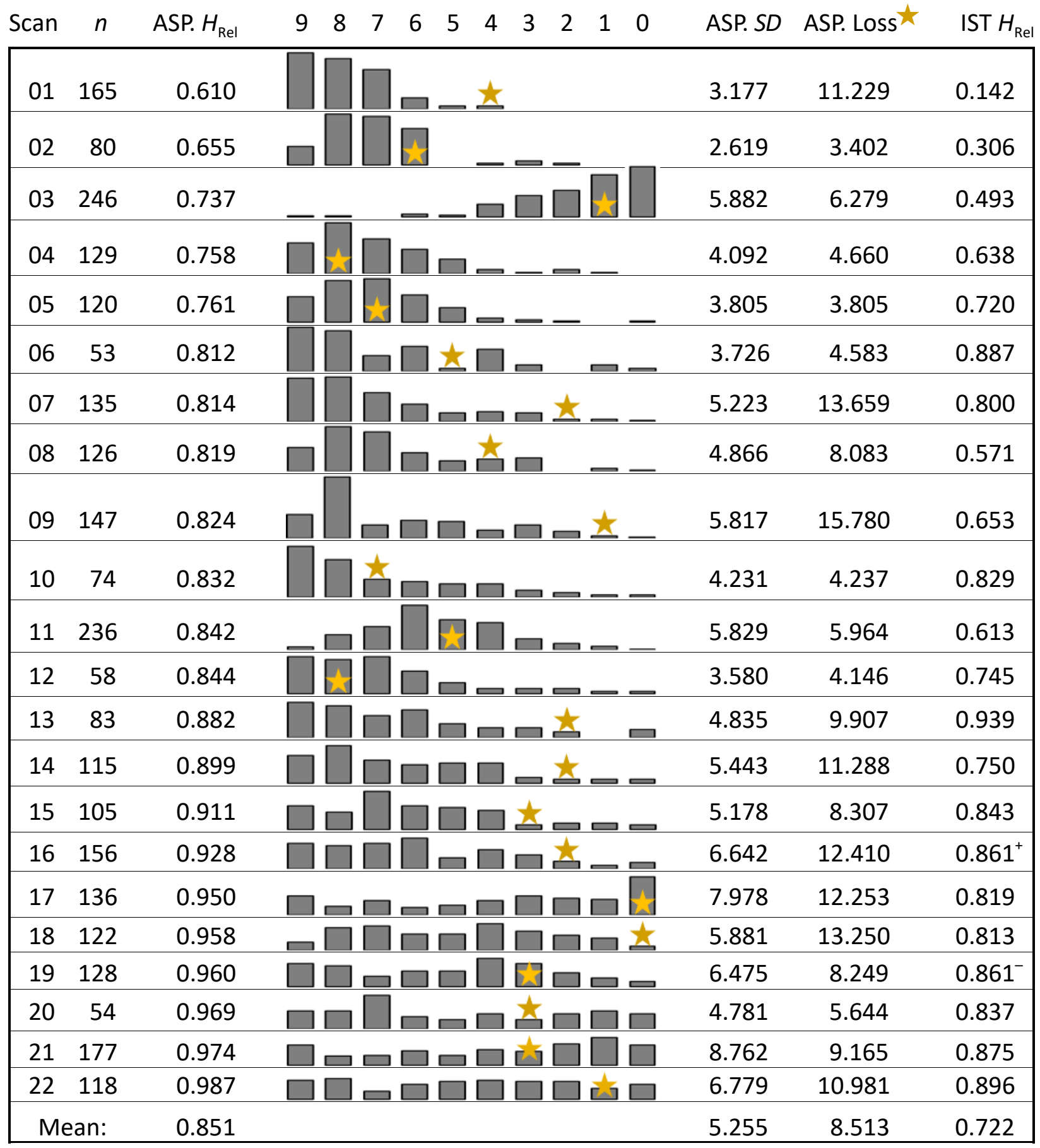

Excerpted and modified from Fig. 6.2, from data kindly provided by JM Wardlaw \& F Chappell. These 22 Excel $^{\circledR}$ charts present probability distributions (each 10-bar total area is 1 unit) by scan among each scan's $n$ abnormal ASP[ECTS] $(<10)$ ratings. Order $\downarrow$ is by increasing ASPECTS entropy. $H_{\text {Rel }}$ is Shannon's relative entropy (entropy $\div$ maximum entropy). $S D$ is standard deviation. Replacing the $S D^{\prime}$ s sample mean by the rsNR's rating $\star$ yields an ASPECTS loss function (\$2.4). 
A probability distribution assigns probabilities $\left(0 \leq p_{i} \leq 1\right)$ to all $N$ possible or conceivable different outcomes of one event (prior to knowledge of the event's outcome) so that they sum to 1, i.e., $\sum p_{i}=1$. Eliminating $N_{\text {elim }}$ outcomes from consideration, e.g., Fig. 6.2's misses, yields a conditional probability distribution (conditional on the information causing this elimination), all of whose remaining $N_{\text {rem }} p_{i}>0$ must be proportionally renormalized ${ }^{181}$ to $\sum p_{i}=1$ - hence, now, the uniform total heights of the bars of $N_{\text {rem }}=10$ abnormal ASPECTS ratings in each of Fig. 6.3's 22 charts. For positive scans, the bars are proportional to the probabilities of each ASPECTS 9-0 rating, given that ASPECTS $\neq 10$.

The sample frequency probabilities might be extrapolated as approximate population probabilities for any population the sample is considered to represent adequately, even if not truly randomly (see the original authors' methods of selecting scans and raters). Each scan has ratings at $\geq 6$ values, with $\geq 9$ for 20 , and all 10 values for 14 , of the 22 scans. A large sample will encounter outliers, ${ }^{\mathbf{1 8 2}}$ eventually populating all bins, so here is a way to concentrate on the common scores. The number of scores that are $\geq 1 / 3$ as probable as the most probable score (subtracting 1 for the largest score itself) is $\geq 3$ for $19, \geq 4$ for $13, \geq 6$ for 7 , and all 9 other scores for 2 scans, usually together more probable than the mode. Most of these strong contenders differ by $\geq 2$ points from each other and the rsNR, all the more worrisome clinically. ${ }^{183}$ Some of this spread is CT's fault - a better scale than ASPECTS (say, IST-3) still yields rather dispersed CT ratings - but not all of the fault is CT's.

To make the original authors' remark on clustering (the inverse of dispersion or spread) rigorous, consider the individual probabilities, $0 \leq p_{i}<1$, of a scan's scores. By definition, the smaller the $p_{i}$, the more surprising its outcome is in the instance when it occurs. It is convenient practically and mathematically to scale this surprise logarithmically as $-\log _{2} p_{i}$, the outcome's surprisal, in bits. ${ }^{184}$ Now for all $N$ of a distribution's $p_{i}$, the surprisals' probability-weighted mean (their expectation) is

Claude Shannon's entropy, $H \equiv-\sum p_{i} \log _{2} p_{i}\left(i=1 \rightarrow N\right.$, labeling the $p_{i}$ of each different value $) .{ }^{185}$

In a distribution where some $p_{i}=0$, their infinite surprisals would seem intractable in $H$-except for an old calculus trick, l'Hôpital's rule, which evaluates $0 \log 0$ as $0 .{ }^{186}$ This expectation formula, the entropy of a probability distribution, provides a mathematically objective measure to compare the dispersions of (or clustering in) probability distributions, at least among those with the same $N$. For uniform $p_{i}$, the ultimate uncertainty for a given $N$ is the maximum entropy, $H_{\mathrm{Max}}=\log _{2} N$ bits. Any ideal test would accurately raise one $p_{i}$ to precisely 1 , reducing uncertainty to $H=0$.

181 Donkin 1851, p. 356, specifying, of the elimination (or any change) of selected $p_{i}$, "without having otherwise any reference to the rest." His Theorem (of stable mutual ratios) may be the first explicit statement of this principle.

182 Deming 1944, pp. 360-2, discusses "Variability in Response" to surveys, which may also apply to this scoring survey. "[D]iffering ideas regarding definitions" (cf. the vague ASPECTS definitions) contribute to response variability, but some respondents do not even report their ages consistently. This is reminiscent of imaging's point-spread function.

${ }^{183}$ For $n=25$, Fahmi et al. 2019, p. 120, Fig. 3, compared 2 raters of supposedly "more accurate" (p. 117) CTASI ASPECTS: they agreed in $20 \%$ and differed by $\geq 2$ in $76 \%, \geq 3$ in $48 \%, \geq 4$ in $32 \%, \geq 5$ in $20 \%, \geq 7$ in $12 \%$, and by 9 points in $8 \%$.

$184-\log _{2} p_{i}$ : implicit in Shannon 1948, p. 11; explicit in Fano 1949, p. 10, Good 1950, pp. 74-5; named by Tribus 1961, p. 64; bit: not a physical dimension but just the scaling unit for base-2 logarithms, originally named by John W. Tukey in 1946 to count discrete binary digits (Tropp 1984, p. 154), then adopted by Shannon 1948 (p.1) for his continuous measure.

185 Shannon 1948, p. 11: the formula and its name, "entropy," and symbol, "H." The "-" sign makes the function positive.

${ }^{186}$ Ross 1980, p. 172; Courant \& John 1989, p. 464; Hairer \& Wanner 1996, pp. 240-3. 
Figs. 6.2 and 6.3 are ordered from left or top by a quantity that can now be stated: the increasing entropy of their positive (9-0) ASPECTS distributions. Although the entropy can be hard to intuit, toward the right or bottom the ASPECTS 9-0 distributions become less clustered-less decisive. This is a little easier to see in Fig. 6.3 with the renormalized conditional probabilities as bar heights, but Fig. 6.2 confirms the original authors' comparison of clustering throughout the range of entropies.

To compare the IST-3 scale $(N=8)$ and ASPECTS $(N=10)$ mathematically, their entropies are divided by their respective $H_{\mathrm{Max}}=\log _{2} N$ for hypothetical utterly even dispersions with no clustering. This yields a relative entropy ${ }^{187} H_{\mathrm{Rel}} \equiv H / H_{\mathrm{Max}}$, independent of $N$. For the 22 rsNR-positive scans, the mean $H_{\mathrm{Rel}}$ are 0.722 for IST-3 and 0.851 for ASPECTS. By the Wilcoxon signed ranks test ${ }^{188}$ the difference between these means is statistically significant at $p=0.0002$. The measured difference between the scales could well be clinically significant too. In this connection it is fitting that entropy has been called "missing information." ${ }^{189}$ From this viewpoint, the respective means' reductions below maximal $H_{\mathrm{Rel}}=1$ reflect the information revealed on average by the scale. These informative increments are 0.278 for IST-3 and 0.149 for ASPECTS. Thus IST-3 acts as a control to set a bar for what can be accomplished by CT. ASPECTS rises barely over halfway to this bar, yielding only $54 \%$ as much information (relative entropy reduction, allowing for the difference in $N$ ) in terms of decisiveness (clustering, agreement) as the IST-3 scale shows to be possible by CT.

A necessary but insufficient condition for reliability is score clustering: that raters agreeminimizing an interrater loss function (\$2.4) while hoping the truth lies within this clustered range (often belied by rsNR in Figs. 6.2, 6.3). From a baseline of complete ignorance denoted by $H_{\mathrm{Rel}}=1$, the IST-3 > ASPECTS inequality in $H_{\text {Rel }}$ change reiterates as comparably and mathematically literally as possible what the original authors pointed out about the poorer clustering in ASPECTS. Yet there is more to say.

Until now we have referred almost interchangeably to two related concepts of imprecision ${ }_{2}(\$ 2.3)$, dispersion and spread. But going forward, we distinguish them mathematically by scale type (\$2.1):

Dispersion allocates ratings to diverse values as a nominal-scale concept irrespective of order. Entropy correspondingly treats the values as nominal because it has no input for any order. ${ }^{190}$

Spread measures how far the values fall from each other, based on at least ordinal distances. Since ASPECTS claims ratio status (for volume), for which \$6.1 offers fleeting support, it is fair to use an interval-based test (but we cannot extend this to the partly nominal IST-3 scale).

${ }^{187}$ Shannon 1948, pp. 14,18. As bits $\div$ bits, relative entropy is a pure number independent of the logarithmic base. Others using "relative entropy" in this original sense: MacKay 1953, p. 18; Barlow 1961, p. 225; Harman 1963, p. 96; Thomas 1969, p. 514. Unfortunately this phrase subsequently appropriated for a different information function that already has other names, namely, the Kullback-Leibler [e.g.] "discrimination information" (Kullback 1987), as if to leave Shannon's relative entropy nameless. In what follows here, probably the precision ${ }_{1}>\operatorname{precision}_{2}(\$ 2.3)$.

${ }^{188}$ Siegel 1956,pp. 75-83+247; Siegel \& Castellan 1988,pp. 87-95+319. The scans are weighted equally and the $p$ is 2 -tailed.

189 Shannon 1948, p. 20; Rothstein 1951, p. 171; Ben-Naim 2008, pp. 19, 21; Grandy 2008, p. 46.

${ }^{190}$ Entropy can also treat values from the higher scales (if discrete, \$2.1) individually as long as the entire data structure need not be considered for information theory's more sophisticated systems-related applications like data compression. 
A standard measure of spread on at least an interval scale is the standard deviation $(S D) .{ }^{191}$ ASPECTS's $S D$ and relative entropy in Fig. 6.3 have only a "strong" Spearman rank-order correlation coefficient of $r_{\mathrm{s}}=0.67 .{ }^{192}$ With a mean $S D$ of $5.255,>1 / 2$ of its total scale, ASPECTS is very spread. (If $S D$ is held inappropriate for so unruly a scale as ASPECTS, Q.E.D.!) The outer range (span) of scores $\geq 1 / 3$ as probable as the mode is $\geq 4$ for $18, \geq 6$ for 10 , and total (10) for 4 of the 22 scans.

Trading the $S D$ formula's $\bar{x}$ for the rsNR's rating makes it a quadratic mean (or root mean square) of a linear loss function, retaining some of quadratic loss's overweighting of larger deviations $(\mathbf{\$ 2 . 4}){ }^{193}$ When the rsNR is eccentric from the mean rating, ${ }^{194}$ mean loss is often $\gg S D$. Dichotomization's concealment of the loss function is part of why unweighted $\boldsymbol{\kappa}$ was specious in $\S 5$. Any reasonable loss function bodes even worse than SD for ASPECTS (by any modality) in stroke research and care.

The available data coordinate ASPECTS with $1 / 3$ MCA ratings only for rsNR but compare all raters' totals. For the $22 \mathrm{rsNR}(+)$ scans, $\mathbf{3 9 . 3 \%}$ of ratings are $>1 / 3$. Common ASPECTS dichotomizations, with their $\%$ on the severe side, are: $8 / / 7,65.1 \% ; 7 / / 6,49.9 \% ; \mathbf{6} / / \mathbf{5}, \mathbf{3 7 . 6 \%} ; 5 / / 4,30.1 \% ; 4 / / 3,21.5 \%$. Thus $6 / / 5$ comes closest to the raters" " $1 / 3$ MCA," but for the rsNR $5 / / 4$ and $4 / / 3$ come closest (Fig. 6.2). By interrater agreement (clustering), too, $6 / / 5$ best matches " $1 / 3$ MCA": $H=0.78$ vs. 0.75 bits. In this sample, $1 / 3$ MCA beats ASPECTS in agreement slightly by raw $H$ and further by reversal on one scan, an "agreement to disagree" not detected by $H$, of which $6 / / 5$ has the fewest. $(H \approx 0.85$ at $8 / / 7$ and $7 / / 6$; while $H$ is lower at and beyond $5 / / 4$, these have many more reversals.) Thus, empirically " $1 / 3 \mathrm{MCA}$ " corresponds closest to ASPECTS 6//5. As Table 6.2 shows the frequency of small and large region involvement to be $\approx$ proportional, $6 / / 5$ sounds like a “2/5" rule, but ASPECTS's any part threshold (\$3.2) overcorrects $6 / / 5$ so that an ASPECTS 5 infarct might involve just $1 \%$ of the MCA territory (\$7). Since ASPECTS spreads widely and we know not how or by what bias raters estimated $1 / 3 \mathrm{MCA}(\$ 4)$, ASPECTS and the $1 / 3$ MCA rule do not reliably elucidate each other or anything else.

Beyond the natural difficulties of CT interpretation, ASPECTS unhelpfully adds more noise. This noise inherent in ASPECTS necessarily also degrades its CT perfusion and MRI scores, whether obtained manually or automatically. An automatic ASPECTS is just one rater, of unknown accuracy or agreement with other automatic raters. Noise is expected from \$6.1 and also because ASPECTS's irregularities are documented and acknowledged in some of its literature and are displayed even if unacknowledged in some of the rest. So far we have reiterated this - perhaps better. But not yet best. A purer study, using DWI, follows in $\$ 6.3$, where $6 / / 5$ measures out as optimally $\approx 1 / 4 \mathrm{MCA}$.

191 §6.1 justifies the use of $S D$ for ASPECTS in this demonstration by suggesting stochastically interval behavior. With $n=259$, we use the sample $S D,\left((1 /(n-1)) \sum\left(x_{i}-\bar{x}\right)\right)^{\wedge} 0.5$, not the population $S D$ with $1 / n$, because our interest is the population of all possible raters. The population $S D$ is the quadratic mean of $\left(x_{i}-\bar{x}\right)$, from which the sample $S D$ substitutes $1 /(n-1)$, or under the square root divides by $(n-1) / n \equiv$ "Bessel's correction" (Kenney 1940, p. 125).

192 Spearman 1904, Siegel 1956 pp. 202-13, Siegel \& Castellan 1988 pp. 235-44, and Corder \& Foreman 2009 pp. 124-33 for $r_{\mathrm{s}}$; Evans 1996 p. 146 for "Strong" "“Very strong" would be $\geq 0.8$; these strength labels are overstated for some jobs).

${ }^{193}$ As with $S D$, for sampling we modify the quadratic mean by retaining "Bessel's correction." Its higher weighting of larger deviations is evident in "the well-known inequalit[y]" (Hoehn \& Niven 1985, p. 151) that quadratic mean > arithmetic mean.

${ }^{194}$ The rsNR's threshold to count a region was not stated, but citations in $\$ 3.2$ note ${ }^{67}$ of any involvement threshold $>0$ come only later ( $\geq 2013$ ), and Fig. 6.3's mean ASPECTS is 3.5 for rsNR vs. 5.8 overall (scanwise, not weighted by $n$ ). 


\section{$\S 6.3$ A Patient-Level Pooling of DWI-ASPECTS Data}

To assess ASPECTS without adulteration, DWI's visualization of cytotoxic edema bypasses plain CT's insensitivity and nonspecificity $(\mathbf{\$ 6 . 2})^{\mathbf{1 9 5}}$ and avoids CTP's reperfusion ( $\$ 9$ note $^{284}$ ). For a meta-analysis of published data, Ke Lin, Constance de Margerie-Mellon, and Julian Schröder sent 949 data pairs underlying their scatter (or box-and-whisker) plots. ${ }^{196}$ Their original plots, and a comparable 217 pairs in an earlier scatter plot, ${ }^{197}$ agree in showing wide scatter, so one of the missions of meta-analysis, to weigh contradictory results, is not at issue here. Rather, it is useful to combine data into nearly 1000 points in order to demonstrate ASPECTS's wide scatter comprehensively in one place, firmly embedded in context. The results appear in Figs. 6.4-6.5 and Tables 6.1-6.2. The scattered relation between semiautomated infarct volume and ASPECTS is easily perceived. We begin with decoding: how to interpret an ASPECTS.

\section{$\underline{\mathrm{mL}}$ Decoding ASPECTS: Volume $(\mathrm{mL})$ as a function of ASPECTS}

400

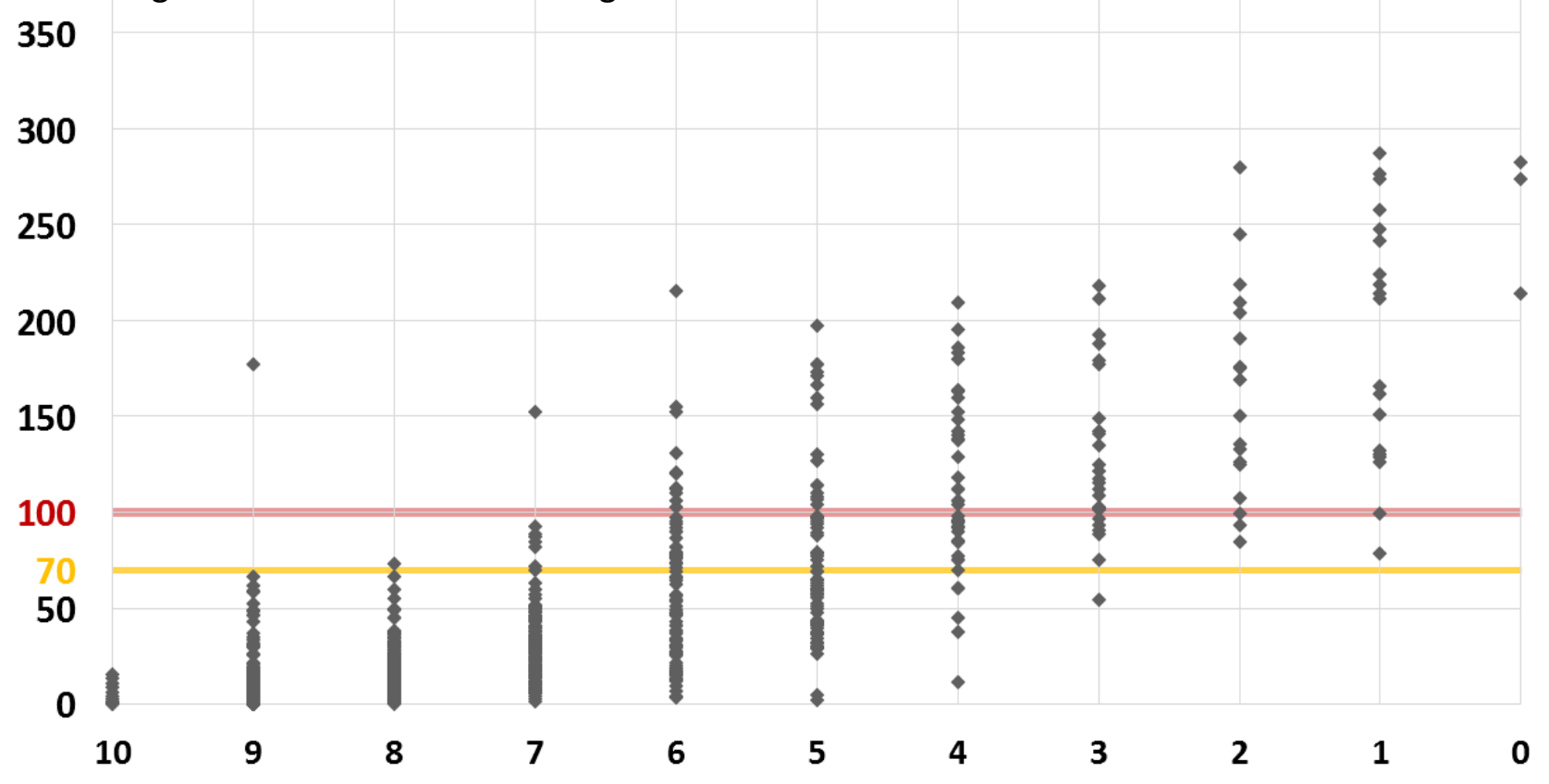

195 e.g., Fiebach et al. 2002, Saur et al. 2003, Amukotuwa et al. 2019, p. 2783-even if DWI still is imperfect: Abbott et al. 2017, pp. 3-4; Gomez 2018, p. 2217.

${ }^{196}$ Lin et al. 2011, p. 230 (pp. 229-30: ASPECTS by consensus of 2 neuroradiologists versus semiautomated volumes); de Margerie-Mellon et al. 2013, p. 3566 (p. 3565: ASPECTS by a stroke neurologist versus semiautomated volumes); Schröder et al. 2014, p. 3585, Figure 2A (p. 3584: ASPECTS by a stroke neurologist versus semiautomated volumes).

${ }^{197}$ Singer et al. 2009, p. 2745. Cf. wide spreads of CT-ASPECTS vs. volume (by CBF) in Haussen et al. 2016 (p. 2320) and Dehkharghani et al. 2016 (p. 1401), and of 3 ASPECTS groups (CT manually, CT automatically, CTP manually) vs. FIV in Sundaram et al. 2019 (p. 2036, with "successful recanalization" minimizing infarct extension in FIV, p. 2037). Cf. similar PWI-ASPECTS scatter and worse PWI-DWI mismatch-ASPECTS scatter in Lassalle et al. 2016 (p. 2556).

198 Data from Lin et al. 2011, de Margerie-Mellon et al. 2013, and Schröder et al. 2014, kindly sent by the lead authors. 
The ASPECTS scale along the $x$-axis reads from 10 to 0 because 10 is the true zero of ASPECTS as no visible pathology. This way the correlation runs in the more customary positive direction. These five replicated scatter plots of 949 data points show how various ASPECTS dichotomizations ${ }^{199}$ rate as measures for a $1 / 3$ MCA rule $\left(100 \mathrm{~cm}^{3}\right.$, pink $)$ or a $1 / 4 \mathrm{MCA}$ rule $\left(\$ 6.2,70 \mathrm{~cm}^{3}\right.$, gold). With respect to either of these lines and the dichotomization, patients in the upper left and lower right quadrants are wrongly classified.

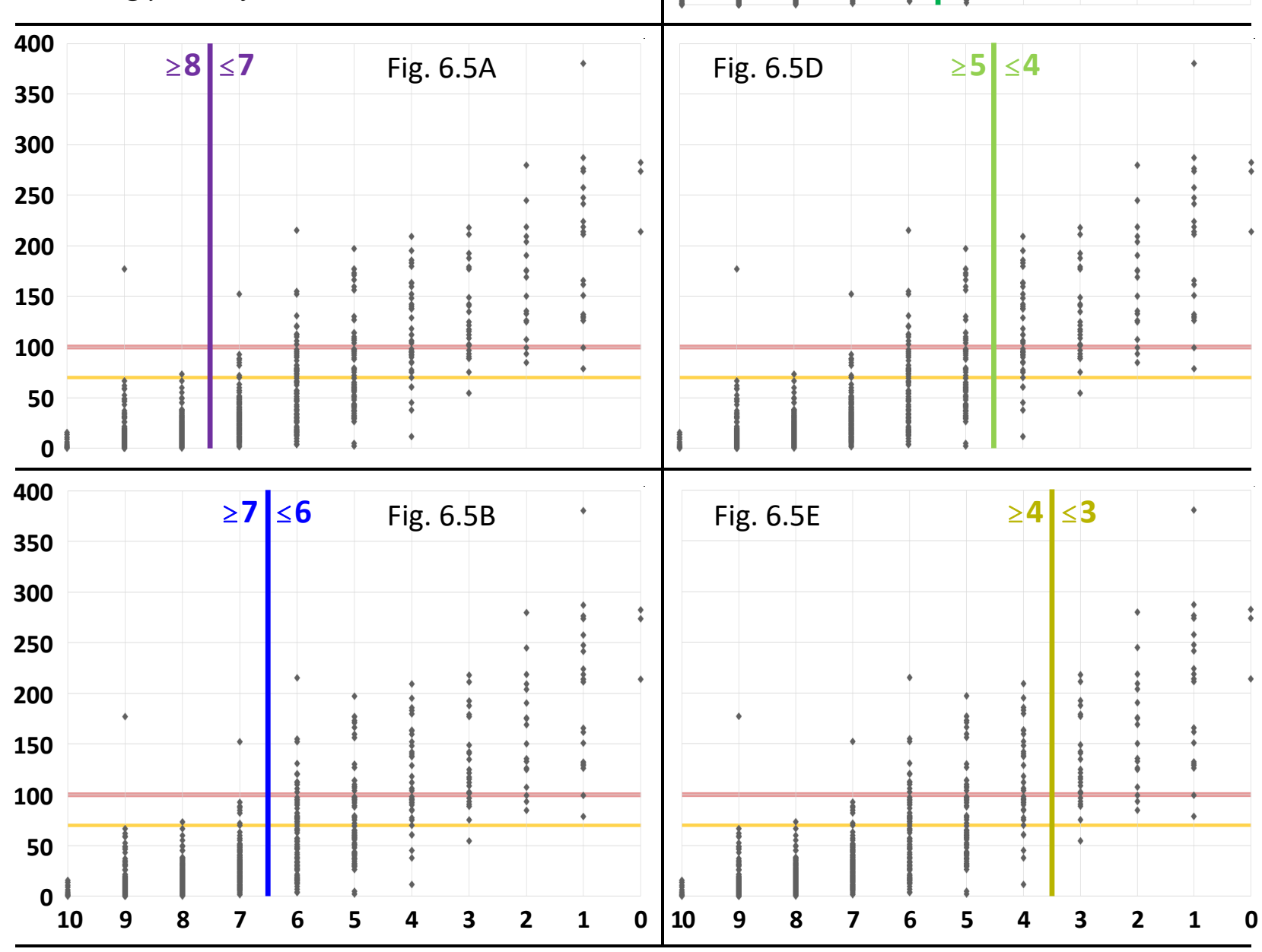

199 This citation list uses §4's “//” notation. For 8//7: Barber et al. 2000, pp. 1671-3; Pexman et al. 2001, pp. 1534-6, 1541; Phan et al. 2006, p. 478 (concluding that "7 does not correspond to one-third of the MCA territory"-p. 480). For 7//6: Pexman et al. 2001, p. 1539 (?); Demaerschalk et al. 2006, pp. 202-3; Schröder et al. 2014, pp. 3585-6. For 6//5: Powers et al. 2015, pp. 3031-2; Goyal et al. 2016a, p. 1728; Powers et al. 2018, p. e71; ACR et al. 2021, pp. 3, 6. For 5//4: Puetz et al. 2009, p. 357; Noorian et al. 2015. For 4//3: Lin et al. 2011, p. 230. Each of these except 4//3 has further examples among §9's thrombectomy trials for enrollment or subgroup analysis. 
Table 6.3 Diagnostic Statistics for Selected ASPECTS Dichotomizations.

\begin{tabular}{|c|c|c|c|c|c|c|}
\hline From 949-point pool & \multicolumn{3}{|c|}{ For Volume $>100 \mathrm{~cm}^{3}$} & \multicolumn{3}{|c|}{ For Volume $>70 \mathrm{~cm}^{3}$} \\
\hline ASPECTS & PPV & NPV & Wrongs & PPV & NPV & Wrongs \\
\hline$\leq 7(8 / / 7)$ & $24.5 \%$ & $99.8 \%$ & $33.8 \%$ & $39.2 \%$ & $99.6 \%$ & $27.4 \%$ \\
\hline$\leq 6(7 / / 6)$ & $38.1 \%$ & $99.7 \%$ & $17.8 \%$ & $58.5 \%$ & $98.5 \%$ & $12.9 \%$ \\
\hline$\leq 5(6 / / 5)$ & $52.6 \%$ & $98.3 \%$ & $10.1 \%$ & $73.7 \%$ & $95.0 \%$ & $9.0 \%$ \\
\hline$\leq 4(5 / / 4)$ & $71.7 \%$ & $96.6 \%$ & $6.2 \%$ & $93.4 \%$ & $91.8 \%$ & $8.0 \%$ \\
\hline$\leq 3(4 / / 3)$ & $83.3 \%$ & $94.3 \%$ & $6.4 \%$ & $98.5 \%$ & $88.3 \%$ & $11.0 \%$ \\
\hline
\end{tabular}

Table 6.4 Decoding ASPECTS (Back-Translating from ASPECTS to Infarct Volume):

Mean and Median Volumes of Selected ASPECTS Values, and Inter-Mean (preferred by the author) and Inter-Median Volume-Threshold Equivalents for Key Dichotomizations.

From 949-point pool

ASPECTS

8

Full Outer

Interquartile Mean

Median

$\frac{n}{188} \quad \frac{\text { Range }\left(\mathrm{cm}^{3}\right)}{0.4-73} \frac{\text { Range }\left(\mathrm{cm}^{3}\right)}{8-23} \frac{\left(\mathrm{cm}^{3}\right)}{17} \frac{\left(\mathrm{cm}^{3}\right)}{14}$

$8 / / 7$ inter-mean or inter-median threshold:

7

154

1 - 152

$17-39$

23

20

$7 / / 6$ inter-mean or inter-median threshold:

30

26

6

95

4 - 215

26 - 77

6//5 inter-mean or inter-median threshold:

\section{5}

69

2 - 197

$43-97$

42

36

5//4 inter-mean or inter-median threshold:

4

40

$12-210$

$85-142$

$4 / / 3$ inter-mean or inter-median threshold:

3

25

55 - 218

101 - 149

54

47

65

55

76

63

94

84

$113 \quad 105$

121

111

129

117

See comments for both tables on next page. 


\section{Comments on Table 6.3}

Infarct volumes and ASPECTS are not dichotomous, but when they are artificially dichotomized they can be shoehorned into having sensitivity, specificity, and positive and negative predictive values. Physicians often recite sensitivities and specificities, ${ }^{\mathbf{2 0 0}}$ but these are the forward probabilities of results. Not until 1966 was it published that to interpret a test result, one wants to know the inverse probability, by which the given result reflects a causal fact. The requisites are instead the positive and negative predictive values: $\mathrm{PPV} \equiv$ true positives $\div$ all positive $t$ ests, and NPV $\equiv$ true negatives $\div$ all negative tests $^{\mathbf{2 0 1}}$ These are properties not just of the test but realistically also of the fact's prevalence. Thus the table's PPVs and NPVs can vary both by random sampling and significantly by clinical or research settings. Yet as a composite of three similar-looking distributions from clinical practices they give a fair idea of the behavior to be expected from ASPECTS. Compared to the $100 \mathrm{~cm}^{3}$ rule, the $70 \mathrm{~cm}^{3}$ rule $(\S 4)$ that de Margerie-Mellon et al.'s original chart also marks is hardly better served.

If ASPECTS $8 / / 7$ targets $100 \mathrm{~cm}^{3}$, in the sample it accepts for treatment just one too-large infarct, but at $177 \mathrm{~cm}^{3}$ this infarct is far too large. This dichotomization's sensitivity (probability a positive will be excluded) comes at the price of such marked nonspecificity (probability a negative will be falsely passed) that $75 \%$ (1-PPV) of the patients excluded from therapy should have been treated. Apparently greater accuracy can be obtained with lower ASPECTS thresholds. But attending only to this raw number implicitly assumes symmetry of wrongs, an unusual loss function (\$2.4, Fig. 2.1) in real life. In thrombolytic therapy, an aversion to causing symptomatic hemorrhage can make the loss function asymmetric. A risk-averse practitioner might prefer to leave more patients untreated in order to avoid a few overtreatments. But best_errors can be reduced by just using volume (\$7).

\section{Comments on Table 6.4}

"Decoding" refers to reading the ASPECTS code to try to discover the underlying situation. If you are told only an ASPECTS, what are you sure of about the infarct volume? Often not much. The Full Outer Range indicates how wildly erroneous ASPECTS can be-its poor validity (\$2.3). The Interquartile Range can be used to trim off outliers, but this method omits half of the patients. Here the middle quartiles still show overlap with each adjacent ASPECTS, just not beyond 1 point. To best guess the volume an ASPECTS represents, say, to interpret a guideline written as ASPECTS when using a patient's actual infarct volume measurement, means and medians are calculated from the meta-analysis. Optimized volume thresholds are interpolated to convert to from ASPECTS (\$9). For example, a $6 / / 5$ threshold's mean mean of $65 \mathrm{~cm}^{3}$ is close to the common threshold of $70 \mathrm{~cm}^{3} .^{202}$ The mean-median difference reflects skew of the "linear" volume measure, suggesting the mean mean as the better interpolation, but the values and harms for which infarct volume is a surrogate might scale sublinearly, compensating for the tail. These mathematical details exceed available precision. However, minimizing raw numbers of misclassifications (as merely an "error counting loss matrix") treats distant errors as equal to near errors and does not minimize loss. ${ }^{203}$

\footnotetext{
200 Smith 1944, p. 17 (sensitivity); Yerushalmy 1947, p. 1435 (sensitivity and specificity).

201 Vecchio 1966. See the famous trap sprung at Harvard by Casscells et al. 1978.

${ }^{202}$ For $70 \mathrm{~cm}^{3}$, Demeestere et al. 2017 prefer 7//6 (based on 59 patients, p. 2249), while Saposnik et al. 2019 assume 5//4.

203 Kristjansson et al. 1996 (p. 1424 quoted, emphasis added-counting, not weighing).
} 
Fig. 6.6. Encoding Volume as $\mathrm{cm}^{3}(\mathrm{~mL})$ into DWI-ASPECTS (How ASPECTS Reads Volume).

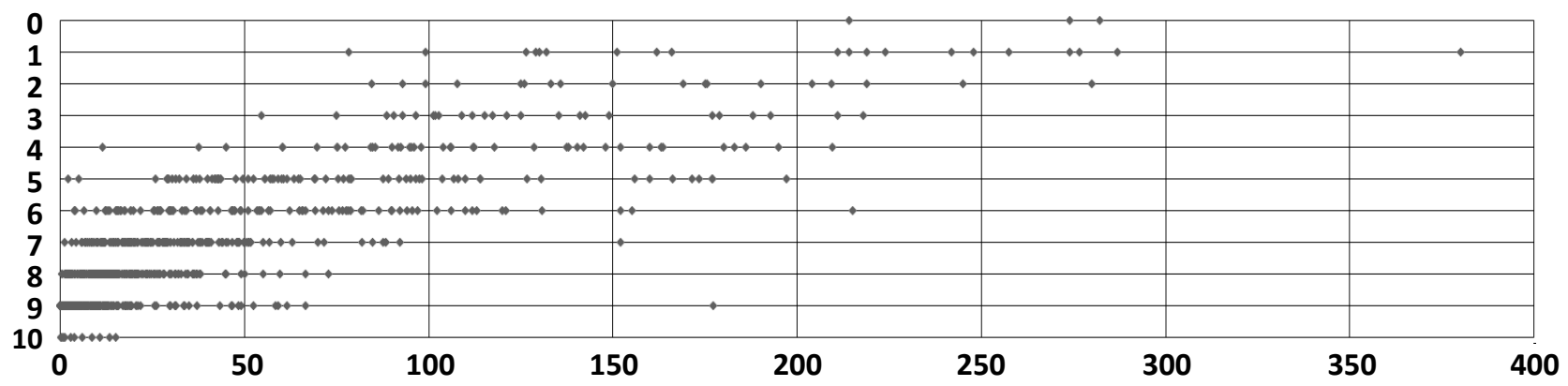

Fig. 6.6's plot reorients the 949 data from the prior pages into prospective functional form with the biologically independent variable, infarct volume, displayed on the $x$-axis. The $y$-axis recognizes 10 as the true zero of ASPECTS. Most $10 \mathrm{~cm}^{3}$ spans of infarct volume have many different ASPECTS, voiding its status as a function and making it a dysfunctional, overlapped "grouping" (binning) of the true volume data. Volumes from 12 to $215 \mathrm{~cm}^{3}$ span at least 6 ASPECTS values, so that, as in $§ 6.2$, "the ASPECTS ... tends to produce widely spread ratings across all [or most] possible scores."204

Such a poor performance can only be expected to degrade research and clinical performance alike. Accordingly, directly comparing automatic outputs for acute infarct CTs from e-STROKE SUITE ${ }^{\circledR}$, ASPECTS lagged substantially behind volume in "Multimodal Predictive Modeling."205

ASPECTS is not a "quantitative scale"206 at Stevens's (\$2.1) highest level, the ratio scale, because identical pairs of scores can form very different volume ratios. This failure reflects its variable intervals in patients caused by its diverse region volumes, so within a patient it is not an interval scale either. Many authors demote its description to "a semiquantitative scale,"207 but this is only a semimeaningful scale category. It has then been claimed to be an ordinal scale, but it is not really that for volume because it shuffles volumes among its scores via diverse combinations of its oddly sized regions. ${ }^{208}$ With its many and large ordinal reversals, ASPECTS has only a very scattered relation to volume. Therefore, with respect to infarcts, ASPECTS is not a quantitative imaging biomarker (QIB). ${ }^{209}$ Mere correlation $(\mathbf{\$ 2 . 5}, \mathbf{\S})$ in a plot does not make ASPECTS safe or effective for individual patients. Table 6.5 shows a taxonomy of ASPECTS's detrimental causes and effects.

204 quoted respectively in this paragraph: Cox 1957, "Note on Grouping" (title); Wardlaw et al. 2010, p. 5.

$205{ }^{\circledR}=$ Brainomix Ltd, Oxford, UK. Brugnara et al. 2020, pp. 3544, 3547, Supp. Figs. II, III, V, and quoting from title (cf. §4).

${ }^{206}$ Barber et al. 2000, pp. 1670-1; Pexman et al. 2001, p. 1534; Mak et al. 2003, pp. 1194-5; Barber et al. 2005, p. 1528.

${ }^{207}$ Demchuk et al. 2005, pp. 2110,2113; Demchuk \& Coutts 2005, p. 416; Dzialowski et al. 2006, pp. 973, 976; Lin et al. 2008, p. 931; Lin et al. 2009, p. 20; Singer et al. 2009, p. 2746 ("a somewhat semiquantitative estimate"); Wusthoff et al. 2011, p. e1551; Gupta et al. 2012, p. 1046; de Margerie-Mellon et al. 2013, pp. 3565-6; Finlayson et al. 2013, p. 234; Hill et al. 2014, Supp.p. 1; Yoo et al. 2014, p. 746; Yoo et al. 2016, p. 685; Jadhav \& Wechsler 2016, p. 242; Lassalle et al. 2016, pp. 2553, 2556: "not originally designed to substitute for volumes"; Wilson et al. 2018, p. 143.

${ }^{208}$ Demaerschalk et al. 2006, p. 203: "a good example of an ordinal scale"; Puetz et al. 2009, p.355: "ordered categorical scale"; Schröder et al. 2018, p. 3: "... the ordinal nature of the scale"; Butcher et al. 2008, p.78: "semiquantitative ordinal." On the shuffling effect disrupting order, cf. Phan et al. 2006, pp. 480-1.

${ }^{209}$ Biomarkers Definitions Working Group 2001, p. 91; cf. Yoo et al. 2012, Sullivan et al. 2015, especially pp. 814-5. 
Table 6.5

A Taxonomy of ASPECTS Pitfalls
(as described in $\$ 3$ and in $\$ 6.1$ )
and Their Categories of Effects

Investigated $^{b}$ (may play a role) in $\S \ldots$

Vagueness of inter-M-region boundaries:

Slice angle change moves boundaries at $M 1 / 2 \& M 2 / 3$ and at $M 4 / 5 \& M 5 / 6$ by shifting them anteroposteriorly, and at M1/4 \& M3/6 by a see-saw effect ${ }^{c} \ldots$

Even if slice angle were standardized, the M-region boundaries, both AP and between levels, are subjective ......

Unsettled definitions of ASPECTS regions: ${ }^{d}$

Caudate body is* vs. is not ${ }^{\dagger}$ noticed and counted separately from $\mathrm{M} 4+5+6^{e} \ldots$

IC excludes* vs. includes ${ }^{\dagger}$ the ALIC ...

$M$ regions include* vs. excludet WM ...

Threshold is "any"* vs. some $\%>0^{\dagger f} \ldots$

Intrinsic to the ASPECTS concept and use:

Categorical scoring of regions ${ }^{g} . \ldots$.

Diversity of region volumes ${ }^{h} \ldots \ldots$.

\begin{tabular}{|c|c|c|}
\hline $\begin{array}{l}\text { For } a \\
\text { patient, } \\
\text { can vary } \\
\text { only scan- } \\
\text { to-scan }{ }^{a}\end{array}$ & $\begin{array}{l}\text { Interrater } \\
\text { variation in } \\
\text { definitions } \\
\text { and visual } \\
\text { estimates }\end{array}$ & $\begin{array}{l}\text { Combinatorial } \\
\text { chance deviates } \\
\text { scores from true } \\
\text { vol. }{ }^{\text {me }} \text { differently } \\
\text { among patients }\end{array}$ \\
\hline
\end{tabular}

$\S 6.2, \S 6.3$

$\S 6.3$

$-\quad \S 6.2, \S 6.3 \quad \S 6.3$

$r$

\section{Notes}

${ }^{a}$ Not a variable on one scan; not investigated (no one was scanned twice at different angles).

${ }^{b} \$ 6.2$ and $\$ 6.3$ investigate their pitfalls collectively and cannot isolate individual effects.

${ }^{c}$ The apparent boundaries move, a well known problem of comparing CT to CT, \& to MRI.

$d^{*}=$ official, ${ }^{\dagger}=$ possible variant (inconsistencies of implementation, $\$ 3.2$ except as in ${ }^{e}$ ).

${ }^{e}$ Unseen if wholly infarcted, but may be moot; mentioned in $\$ 8$, others in prior sections.

${ }^{f}$ The latter, inherently subjective, could be fudged (un)consciously toward a truer volume, increasing interrater variation but perhaps tending on average to mask the other pitfalls.

${ }^{g}$ This problem related to ${ }^{f}$ causes variable amounts of systematic error (\$2.4), a bias.

${ }^{h}$ To the degree regions are affected evenhandedly, volume variations cause random noise, and any small-/large-region frequency bias (cf. Table 6.2) adds systematic error. 


\section{§7. How To Measure Volume: $A B C / 2,2 S h / 3$}

The original target of ECASS and ASPECTS was infarct volume (\$3.1), so let's just measure it. "A stone of any shape becomes increasingly similar to an ellipsoid as the water wears away at it,"210 and so with inspection and a modicum of imagination an infarct of almost any shape appears increasingly amenable to approximation by an ellipsoid with usually distinct major, middle, and minor axes. ${ }^{211}$

The formula for a sphere's volume, $(4 / 3) \pi r^{3}$, readily generalizes to $(4 / 3) \pi r_{1} r_{2} r_{3}$ for an ellipsoid ${ }^{212}$ or, in diameters, $\pi d_{1} d_{2} d_{3} / 6$ ("Tada's formula"213). Within clinical precision, $\pi \approx 3.14 \approx 3$, leaving $d_{1} d_{2} d_{3} / 2$. By CT this formula was first applied to lacunar infarcts (without notation) and to thalamic hemorrhage in the notation " $\mathrm{X} \cdot \mathrm{Y} \cdot \mathrm{Z} / 2$. ." 214 By 1994 the notation changed to "ABC/2." 215 Soon this notation was popularized in "The ABCs of Measuring Intracerebral Hemorrhage Volumes," explaining that "A is the greatest hemorrhage diameter by CT, B is the diameter 90 degrees to A, and C is the approximate number of CT slices with hemorrhage multiplied by the slice thickness" ${ }^{\text {"216 }}$ (more strictly, the center-to-center interval). Now we measure the third perpendicular axis on a reformatted image, but if slices must be counted, subtract one if the highest and lowest are not involved to full thickness. ${ }^{217}$ Axis lengths should not be excessively maximized into narrow spits of infarcts.

Some methods omit the $3^{\text {rd }}$ dimension. The ICE method: Idealize a typical MCA territory, enClose the infarct, and Estimate the area ratio ${ }^{218}$ (presumably where the infarct is largest) - echoes $\$ 4 \# 2$. It is claimed that an $A B$ index discriminates better than $A B C / 2$ for a $70 \mathrm{~cm}^{3}$ or $100 \mathrm{~cm}^{3}$ dichotomization. ${ }^{219}$ But, except perhaps in uniform columns, there is more volume information in 3 dimensions than in 2. Beware of any method, like ICE's cylindrical assumption, that purports to prove otherwise.

\footnotetext{
${ }^{210}$ Hilbert \& Cohn-Vossen 1932/1952, p. 13.

${ }^{211}$ Hilbert \& Cohn-Vossen 1932, p. 12: “'grosze', ‘mittlere', und 'kleine' Achse des Ellipsoids.” In — 1932/1952, p.13, "mittlere" is translated as "mean," but on both etymologic and semantic grounds "median" is more correct.

212 e.g., Pearson 1983, pp. 80-1; Broderick et al. 1993, p. 988; Newman 2007. Letters used for mathematical quantities are italicized, unless embedded in an italicized passage or quoted in roman type.

${ }^{213}$ Zhao et al. 2009, p.1032: " $\pi / 6 a b c . "$ Is $a b c$ in the numerator or the denominator? Contrary to "The standard convention" (Strogatz 2019) for the order of operations, and unlike coequal + and-, the Physical Review Style and Notation Guide (Waldron et al. 2011) evaluates multiplication before division (p. 21) — cf. Feynman et al.'s (1963, pp.6-7) “ $1 / 2 \sqrt{ } n$ " $=1 /(2 \sqrt{ } n)$ and Hakonen \& Lounasmaa's $(1994$, p.1825) " $S / R \ln 2 "=S /(R \ln 2)$. We safely leave division for last here and in $2 S h / 3$ (below).

${ }^{214}$ respectively: Nelson et al. 1980, p. 494; Kwak et al. 1983, p. 494. Cf. Franke et al. 1988 ("The Best Fit Method," cf. Fig. 7.2) and Lisk et al. 1994, p. 134, for bleeds, both citing Kwak - but without mathematical notation.

215 Broderick et al. 1994, which is cited (without notation) as "the Broderick method" by Mendelow et al. 2005 (p. 390) and - 2013 (p. 401), who rely on it to measure and classify hematomas in the STICH trials; cf. Webb et al. 2015.

${ }^{216}$ Kothari et al. 1996 (quoted respectively: title, abstract). (No relation to the clinical transient ischemic attack scale $\mathrm{ABCD} 2$ of Rothwell et al. 2005). $A B C / 2$ 's interrater reliability is reported favorably, e.g.: "outstanding" (Beslow et al. 2010, p. 691); "difference ... clinically insignificant" (Dsouza et al. 2019, pp. 1818, 1821).

${ }^{217}$ Kothari et al. 1996 approximate this correction by excluding slices whose hematoma area is less than $25 \%$ of the maximum hematoma area, and otherwise counting as half a slice those whose area is less than $75 \%$ of the maximum, but Krishnan et al. 2015 and Lee et al. 2016 find this modification to overcorrect. Lee et al. reserve "ABC/2" for Kothari et al.'s method, call Kwak et al.'s original method "simple," and confusingly denote the latter as "sABC/2."

${ }^{218}$ Silver et al. 2001, pp. 114-5, not citing Dippel et al. 2000; Demaerschalk et al. 2006 report $\boldsymbol{\kappa}=0.72$ for ICE and ASPECTS.

${ }^{219}$ Fiebach et al. 2015, implausibly based on few $(n=108)$ mostly small (median $13 \mathrm{~cm}^{3}$ ) infarcts.
} 
The geometric approximation $\boldsymbol{A B C} / \mathbf{2}$ applies also to tumors ${ }^{220}$ subdural and epidural hematomas, ${ }^{221}$ brain volume, ${ }^{222}$ and, key for us, acute cerebral infarcts. Six sets of infarct investigators conclude that:

The A.B.C/2 formula should be applied routinely to $\mathrm{CI}$ [acute cerebral infarct] volume determination based on its low cost and fast calculation; ${ }^{223}$

[The] $0.5 \times \mathrm{A} \times \mathrm{B} \times \mathrm{C} \ldots$ method 4 ( 3 diameters ...) ... simple method acceptable for investigators .... ${ }^{224}$

$\mathrm{ABC} / 2$ is reproducible, is accurate, and provides the best simple geometric estimate of infarction and mean transit time volumes; ${ }^{225}$

[T] $]$ he $\mathrm{ABC} / 2$ method can be used to obtain a fast assessment of volume of the infarct provided its advantages and limitations are clearly understood; ${ }^{226}$

The $\mathrm{ABC} / 2$ method is highly reliable and accurate for quantifying the ... MR imaging-determined mismatch and therefore is a potential tool to quickly calculate a treatable mismatch pattern.... $\mathrm{ABC} / 2$ $\ldots$ is an alternative method when automated mismatch methods are not available, ${ }^{227}$

[I]nfarct volume (CBV) and penumbra volume (MTT) can be reproducibly and reliably calculated using the $\mathrm{ABC} / 2$ formula on $\mathrm{CT}$ perfusion; ${ }^{228}$

As further endorsements, $A B C / 2$ is relied on in additional hemorrhage ${ }^{215}$ and infarct ${ }^{229}$ research.

Even as it improves on "the 'eyeball' technique"(\$4) and ASPECTS (\$6), $A B C / 2$ has some of its own instructional points to clear up. For instance, a rule is sometimes added to take $B$ from the same image as $A^{230}$ instead of allowing use of the largest $B$ if from another image, but use of the same image is probably usual anyway. The denominator of 2 might be challenged if the lesion is jaggedly contoured. But even for supposedly relatively jagged hematomas occurring with warfarin, $A B C / 3$ tended to overcorrect, leaving others satisfied with $A B C / 2$ for warfarin hematomas. ${ }^{231}$ Haley et al. compare $A B C / 2, A B C / 2.4, A B C / 3$, and a formula they call " $2 / 3 \mathrm{SC}$ " ( $2 S h / 3$, next page) for different categories of hematomas, for most of which they prefer $A B C / 2.4$ and " $2 / 3 \mathrm{SC}$."232

220 e.g.: Sreenivasan et al. 2016 (gliomas); Bathla et al. 2017 (vestibular schwannomas).

221 SDH: Gebel et al. 1998, Won et al. 2018; EDH: Hu et al. 2016. Imagine a crescentic SDH sheared straight (Recap 7.1).

${ }^{222}$ Kleinman et al. 2011 (pediatric use).

223 Gómez-Mariño et al. 2001, p. 380; p. 382: high correlations with planimetry (not reported as scatter plots).

224 van der Worp et al. 2001, pp. 425, 426, 429 (“... who do not have access to an image analysis system”).

${ }^{225}$ Sims et al. 2009, p. 2104; 2107: impressive scatter plots, intuitively in $\mathrm{cm}^{2}$.

${ }^{226}$ Pedraza et al. 2012, p. 158; the main limitation was overestimation by what appears, amidst difficulties in the presentation, to be a mean increment of $83 \%$ among a small population of 93 patients.

${ }^{227}$ Luby et al. 2013, pp. 1901 \& 1906; p. 1905: "mismatch volume ... by the $\mathrm{ABC} / 2$... is equivalent to the planimetric .... This is ... the largest ischemic stroke study [193 patients] looking at MR ... mismatch by both $\mathrm{ABC} / 2$ and planimetric ...."

${ }^{228}$ French et al. 2014, p. 235; Table 3 on p. 235 and the scatter diagrams on p. 236 show this is more true for CBV.

${ }^{229}$ Pantano et al. 1999, pp. 502-3; Vogt 2012, pp. 1267, 1271; Marsh et al. 2013, p. 964, noting "its ease of calculation and generalizability in clinical practice"; Marsh et al. 2016, p. 3; Schwamm et al. 2018, p. 982; Byrne et al. 2019, pp. 322,326; Maradi et al. 2019. $A B C / 2$ is accepted matter-of-factly in the "Code Stroke Primer" by Potter et al. 2019, p. 1718.

230 implied by "largest" area: Broderick et al. 1993, p. 988; Gómez-Mariño 2001, p. 381 ("No desenho com maior área ...""On the image with the greatest area ..."); Sims et al. 2009, p. 2105; Pedraza et al. 2012, p. 156; Luby et al. 2013, p. 1902.

$231 A B C / 3$ : Huttner et al. 2006; satisfied with $A B C / 2$ : Sheth et al. 2010, also denying that warfarin hematomas are more jagged; cf. more generally Zhao et al. 2009, Haley et al. 2018. Overlooking "best fit" ${ }^{\text {"214 }}$ for $A B C / 2$ will tend to overestimate.

${ }^{232}$ Haley et al. 2018. $A B C / 1.9$ would correct for " $\pi=3$." Increasing the denominators corrects for using greatest diameters instead of "best fit." ${ }^{14}$ For "2/3SC," see note ${ }^{213}$ on order of operations: using $h$ for C, we'll safely write $2 S h / 3$. 


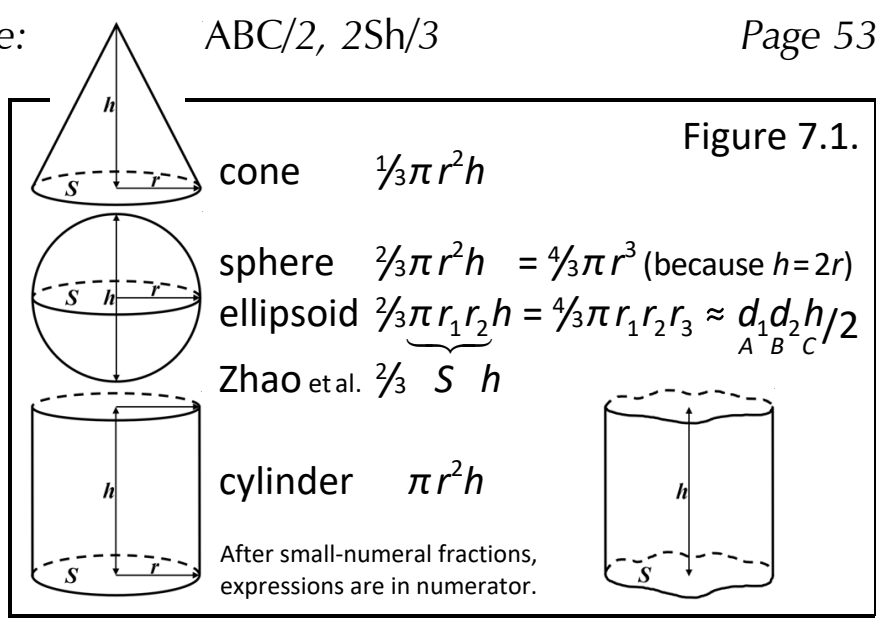

Archimedes gave the volume of a sphere of radius $r$ as 4 times that of a cone of radius and height $r .{ }^{233} \mathrm{~A}$ cone, sphere, and cylinder each of radius $r$ and height $h=2 r$ therefore have volumes proportional to 1:2:3 (Fig. 7.1).

From the ellipsoid formula, Zhao et al. isolate $\pi r_{1} r_{2}$, the area of the widest slice, " $S$," of pathology. ${ }^{234}$ The perpendicular axis to this slice is $h$, the $C$ of $A B C / 2$. Hence $\mathbf{2 S h} / \mathbf{3}$.

For a true ellipsoid, $A B C / 2$ underestimates $\pi$ and volume by $4.5 \%$, while for ideally chosen axes $2 S h / 3$ is exact (same as $\pi A B C / 6$, above). But $A B C / 2$ 's smooth outer contour covers up indentations in its objects, while providing only its blanket adjustment of $-4.5 \%$. With greater irregularity it progressively overestimates volumes, ${ }^{235}$ although rarely if ever as badly as ASPECTS often does (§6). Adjusting $A B C / 2$ 's denominator, as tested above over the range of 2-3, merely shifts the bias.

The solution is the flexibility of $2 S h / 3$ 's customized area measure. The area of the largest slice of lesion takes irregularity into account. $2 S h / 3$ measures volume well, and better than the other formulas. ${ }^{236}$ In probably "less than 1 minute," 237 2Sh/3 offers two strategies. (1) Outline it manually on a system that measures an irregular area. (2) Superimpose an ellipse, matching their major axes in orientation and length, and adjust the ellipse's minor axis to estimate equal areas wrongly included and excluded (Fig. 7.2, "best fit"214). The minor axis is a better $B$ for a flexible $A B C / 2$, if preferred. Don't be too fussy-it already beats ASPECTS. A principle of isovolumic contour variation, shown by the crenellated cylindroid, extends to conoids and spheroids (with crenellation tapering in parallel planes toward the apex or poles) as a conceptual model approximating the more general irregularity.

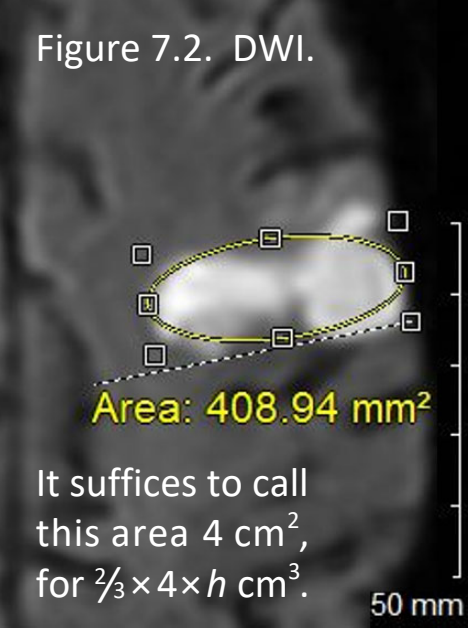

Multidetector CT offers triplane choices for $A$ or maximal $S$, with direct measurement of $C$ or $h$. $C$ or $h$ must be perpendicular to the $A B$ or $S$ plane, not oblique for a greater (partly redundant) diameter. For multiple or highly lobulated infarcts, each infarct or lobe can be measured and they can be added. ${ }^{238}$ After Recap 7.1 (next page), we illustrate in Fig. 7.3 $A B C / 2$ for an estimated MCA territory whose true outline is, as usual, unknown. Then follows a CT and DWI infarct example (Fig. 7.4).

\footnotetext{
233 Archimedes ca. 225 BCE, Book I, Proposition 34.

${ }^{234}$ Zhao et al. 2009: " $S$ " seems to come from "slice" (p. 1032).

${ }^{235}$ Haley et al. 2018.

${ }^{236}$ Zhao et al. 2009; Zhao et al. 2010; Yan et al. 2013; Yang et al. 2013; Haley et al. 2018.

237 as promised for $A B C / 2$ by Kothari et al. 1996 and reiterated by Maradi 2019, p. B147. I find it takes $\approx 30$ seconds.

238 e.g., Vogt et al. 2012, p. 1267: “just added."
} 


\section{Recap 7.1. Recapitulation of Ellipsoidal Methods of Volume Measurement}

Multiplication is vexation, Division is as bad;

The Rule of Three doth puzzle me, And practice drives me mad. ${ }^{239}$

We are past the era of vexation by multiplication and division. We all learned ciphering well beyond the rule of three and now have electronic calculators on pocket telephones or adjacent computers. But in our medical practices we need practice. This includes rehearsing $A B C / 2$ and $2 S h / 3$ on sectional imaging at a suitable modest precision. As "time is brain!" volume calculation prompts a warning: "The enemy of Good is Better." ${ }^{240}$

If an object approximates a rectangular solid, calculate its volume from its edge lengths. But most infarcts, bleeds, tumors are measured best by an ellipsoidal approximation amenable to $A B C / 2$ or ideally $2 S h / 3$. Crescents (like subdural hematomas) not amenable to $2 S h / 3$ can be isometrically straightened for $A B C / 2$, since by Cavalieri's principle shearing parallel planes does not change their aggregate volume (https://en.wikipedia.org/wiki/Cavalieri\%27s principle). Think of shearing a stack of coins or, in two dimensions, taking the area of a parallelogram. By this principle, the third axis is perpendicular to the first two (to avoid double-counting part of $A$ or $B$ ). The ellipsoidal approximation assumes that the irregularities compensated by "best fit" are proportional to the smaller cross sections through most of the object's bulk. Where this theoretically fails, toward the poles, the areas are small and the errors trivial.

Modern triplane CT offers a choice for (approximately) the largest-area profile. From this, choose the longest axis. A PACS probably has a measuring tool with orientable ellipses. Rotate the ellipse and stretch its length to equal (superimposed on) the infarct's long axis. Adjust the width to include as much noninfarct as infarct is excluded (area-matching, but don't fuss too much). If calculating as $A B C / 2$, the compensated ellipse's minor axis is a better $B$ (but then correct upward by $5 \%$ for $\pi / 3$ ). A PACS probably offers an area measure, although perhaps not by default until selected. If area is in $\mathrm{mm}^{2}$, divide by 100 for $\mathrm{cm}^{2}$. The area, $S$, compensates for the object irregularities that cause an overstrict (maximal, not best fit) $A B C / 2$ to overestimate volume. If the PACS computes areas of irregular outlines, tracing these on involved sections and adding them up takes extra time for little further profit.

For DWI (or CT) without recons, take the difference between the highest and lowest sections' positions (making sure that neither or both are labeled "-"). Add one section thickness only if the end sections seem to be involved to their full thicknesses. If only one of the positions is labeled "-," then add the absolute values. Work in $\mathrm{cm}$ or $\mathrm{cm}^{2}$ and multiply for $\mathrm{cm}^{3}$. And remember the factor of $1 / 2$ or $2 / 3$.

239 Halliwell 1842, p. 135, 1844, p. 48, modernized from Davies 1840, p. 18, which cites a manuscript from ca. 1570.

240 quoted respectively: Gomez 1993 (cf. Gomez 2018, a monotonic decay highly variable in time and among patients); Montesquieu 1725-1755/1899, p. 413: "Le mieux est le mortel ennemi du bien" ("Better is the mortal enemy of Good"), shortened by Voltaire 1772, p. 3, to “le mieux est l'ennemi du bien.” But cf. Bivard \& Parsons 2018 on too much haste: "Tissue is more important than time" (title), opposing "nonthinking, faster treatment at all costs" (p. 26). §7 invokes the Pareto principle (https://en.wikipedia.org/wiki/Pareto principle) that a small initial effort yields most of the accuracy. 
$A B C / 2$ estimate of MCA territory volume: $11.5 \times 5 \times 10.5=302 \mathrm{~cm}^{3}, 6 \%$ larger than average in $\S 4$.
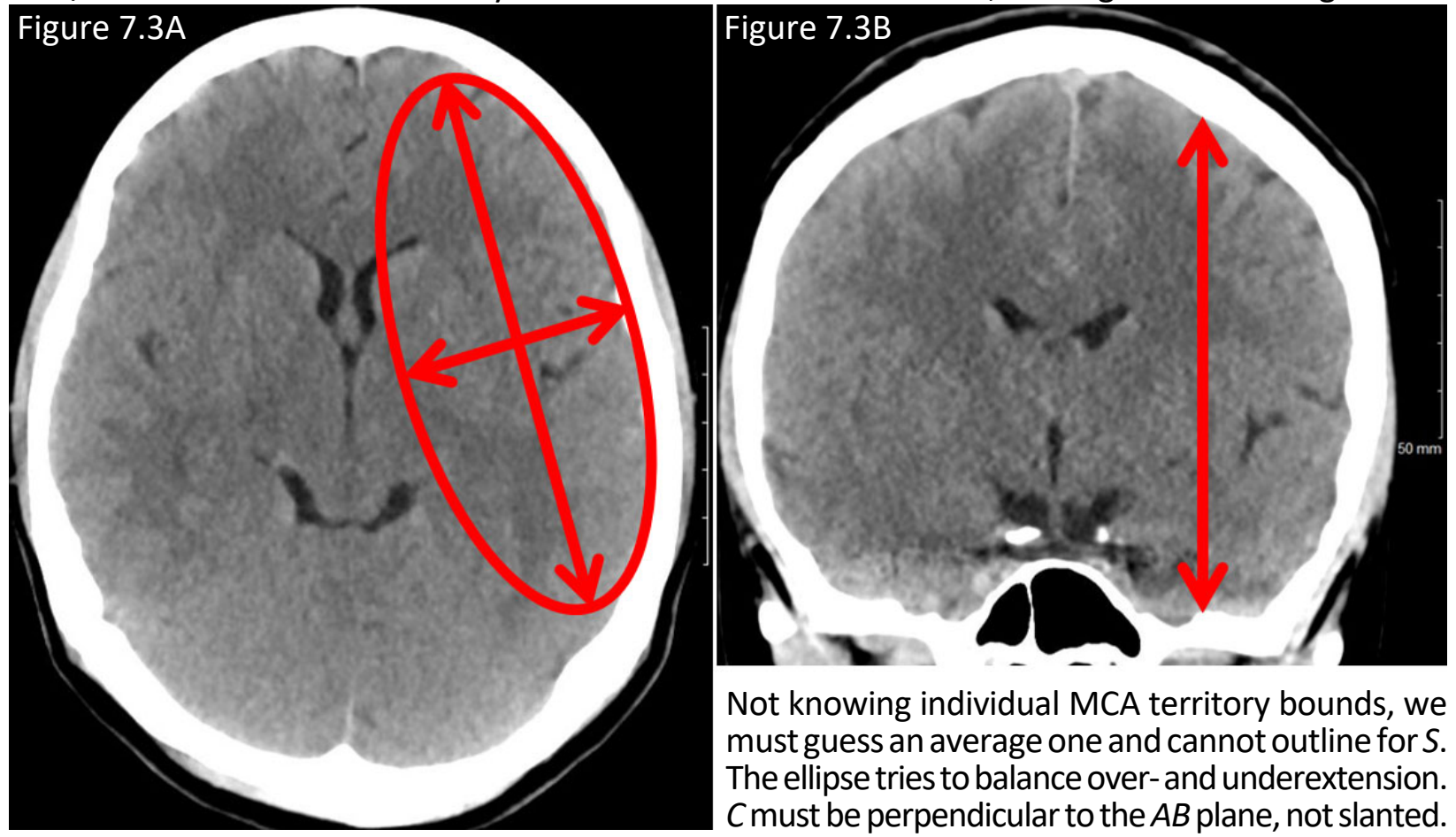

Not knowing individual MCA territory bounds, we must guess an average one and cannot outline for $S$. The ellipse tries to balance over- and underextension. $C$ must be perpendicular to the $A B$ plane, not slanted.

$A B C / 2$ insula+lC+ganglionic estimate: $4.5 \times 3.3 \times 3.5=26 \mathrm{~cm}^{3}$ (could be individual variation from $\S 6$ ).
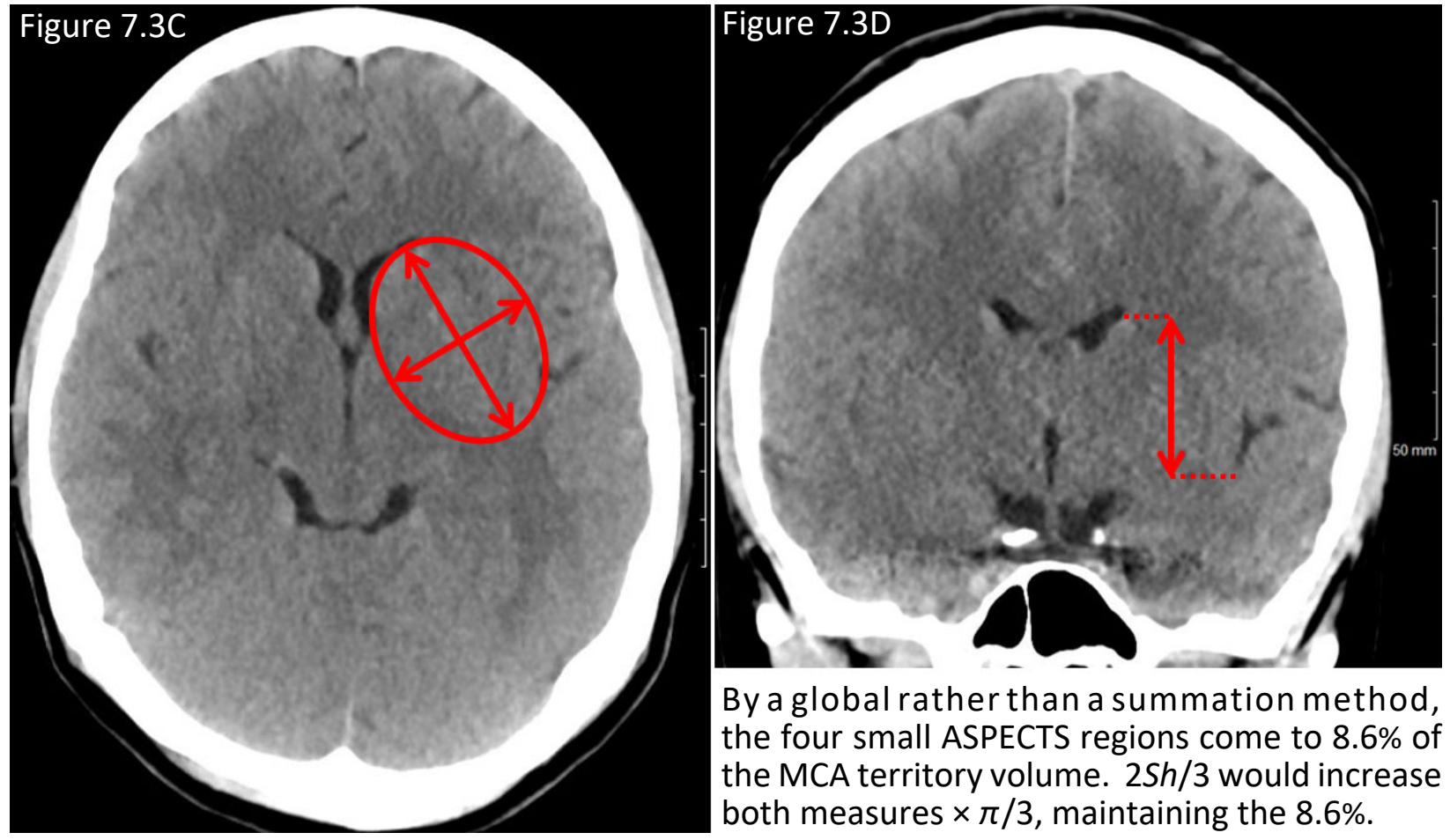

By a global rather than a summation method, the four small ASPECTS regions come to $8.6 \%$ of the MCA territory volume. $2 \mathrm{Sh} / 3$ would increase both measures $\times \pi / 3$, maintaining the $8.6 \%$.

Carefully applied, $2 S h / 3$ and $A B C / 2$ hew closer to the true volume than does ASPECTS (\$6). The acute infarcts on the next page are assessed on the following page by ASPECTS and $A B C / 2$. 
§7. How To Measure Volume: ABC/2, 2Sh/3
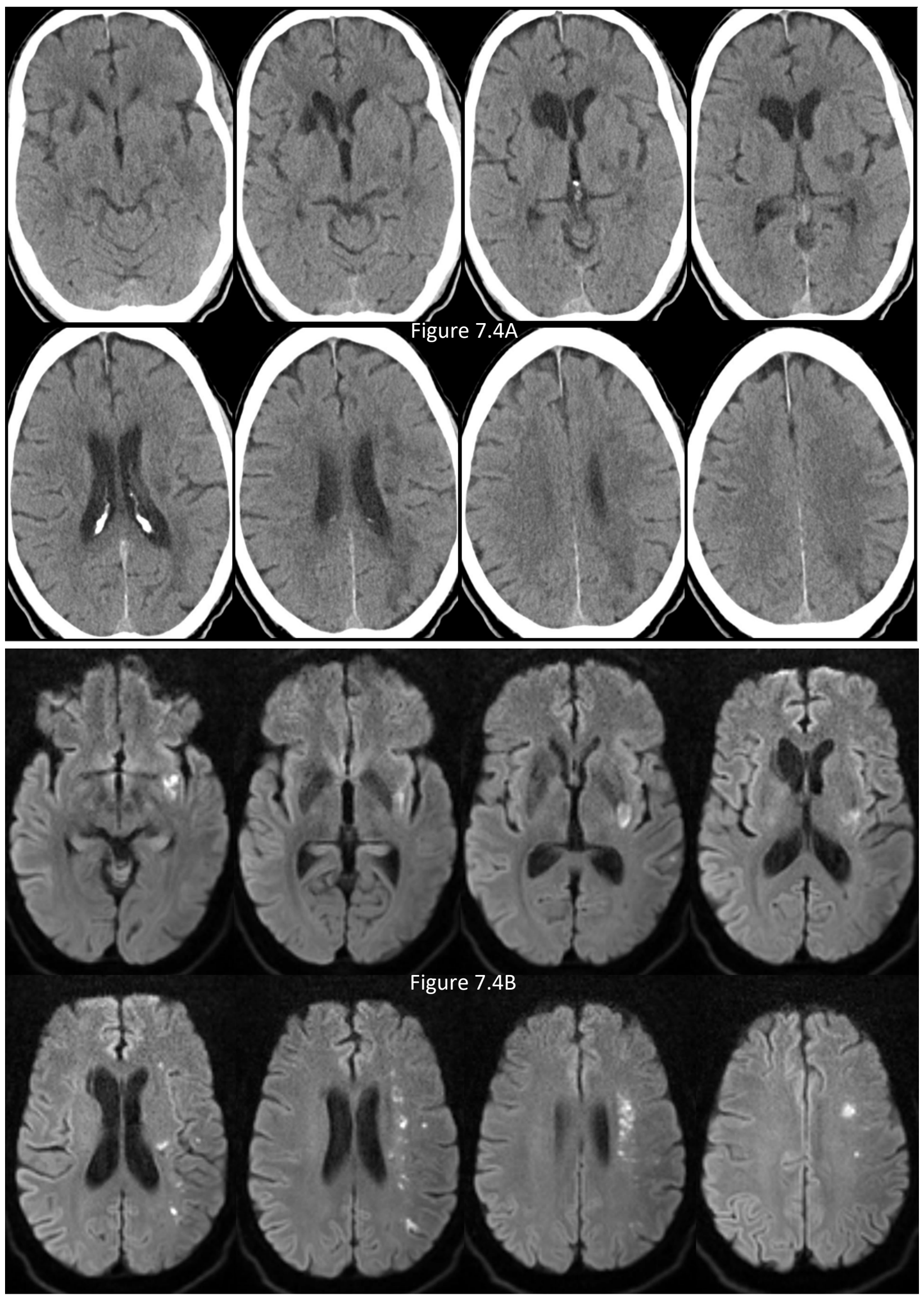
The patient in Fig. 7.4, weak on the right, was interpreted on-call as CT-ASPECTS $=6$, deducting points for insula, internal capsule, M1, and M6. The MRI staff reading the DWI (not scoring for ASPECTS) diagnosed involvement of insula, putamen, and frontal and parietal lobes. Where a $5 \mathrm{~mm}$ lateral lobe on the lowest CT and DWI sections extends subinsularly from the putamen, across the low external capsule, claustrum, and extreme capsule, $\$ \mathbf{6}$ assigns this to the insula because the founding literature assigns these structures nowhere else. ${ }^{241}$ The M-zone involvement is only supraganglionic, M4-6. Going by "an area of early ischemic change" in a region without requiring any positive minimum to be involved (\$3.2), these lesions subtract down to DWI-ASPECTS $=5$.

True volume involvement is a different story. The DWI sections are $5 \mathrm{~mm}$ thick with $10 \%$ gaps. The four putaminal $5 \mathrm{~mm}$ sections and three $10 \%$ gaps strictly total $2.15 \mathrm{~cm}$. Basal ganglionic (mostly putaminal - mainly posterior and some inferior) involvement on the upper (anatomically lower) DWI row calculated by $A B C / 2$ comes to $1.6 \times 0.9 \times 2.15 / 2=1.55 \mathrm{~cm}^{3}$. The M4-6 lesions can be measured as the sum of the largest one (as a sphere) on the highest section added to the product of the estimated mean volume of the others times their apparent number (generously about 50). This measurement comes to $(0.8 \times 0.8 \times 0.8 / 2)+50 \times(0.2 \times 0.2 \times 0.2 / 2)=0.3+50 \times 0.004=0.5 \mathrm{~cm}^{3}$. Even adjusting these estimates, it would be hard to push the $\approx 2 \mathrm{~cm}^{3}$ total to over $3 \mathrm{~cm}^{3}$ (still $\approx 1 \%$ ). With barely $1 \%$ of the average MCA volume involved, the probability of sICH is very low.

Compare this case to the data in $\$ 6.3$ : of the 69 DWI-ASPECTS $=5$ cases, $57 \%$ were $<70 \mathrm{~cm}^{3}$, two just 2.2 and $5 \mathrm{~cm}^{3} .{ }^{242}$ The 2018-19 Guidelines (\$9) tend to deny thrombectomy to these $57 \%$ only because of their ASPECTS. Perhaps some interventionists right such wrongs by nudging the easily fudged ASPECTS toward their intuition (or measurement) of the true volume.

As a cosmetic artifact of notation, the $\approx 2$-digit precision $_{1}(\$ 2.3)$ of $A B C / 2$ or $2 S h / 3$ will allow nominal interobserver disagreements by small amounts that ASPECTS's coarse scale covers up. ${ }^{243}$ Counts of disagreement events are not valid comparisons of imprecision, especially with unbalanced precision $_{1}$. Agreement comparisons must reflect the sizes of disagreements (\$5) on a common footing. Even as $A B C / 2$ and $2 S h / 3$ will differ slightly from each other too, they are not prone to the clinically ominous huge deviations from infarct volume that often plague ASPECTS (\$\$6.2, 6.3). Bypassing ASPECTS's inherent structural defects (\$3, §6.1), $A B C / 2$ and $2 S h / 3$ are more nearly perfectible with training and practice. Relative conformity in ASPECTS within teams who have consciously or unconsciously evolved their own conventions ${ }^{244}(\$ 3)$ may build false confidence based on agreement, but what's the use of agreement when it's inaccurate?

${ }^{241}$ Cf. thick insula in region diagrams in Farzin et al. 2016, Supp. p. 5, Fig. e-1, and in Reidler et al. 2019, p. 454, Fig. 2. Kuang et al. 2019, p. 34, Fig. 1, simply excludes the claustrum and adjacent capsules from consideration.

${ }^{242}$ Also (but apples:oranges): Kim et al. 2016, of 5 CT-ASPECTS $=5,2$ had $47-49 \mathrm{~cm}^{3}$ CTP core; Haussen et al. 2016, of CT-ASPECTS $<6,26(8 \%)$ had $\leq 50 \mathrm{~cm}^{3} \mathrm{CTP}$ core (unsaid how many $\leq 70 \mathrm{~cm}^{3}$ ); Qiu et al. 2020 (p. 643, Fig. 5), even with $20 \%$ thresholds $\left(\$ 3.2\right.$ note $^{67}$ ), of 24 DWI-ASPECTS $<6, \approx 65 \%$ had $<70 \mathrm{~cm}^{3}$ core.

${ }^{243}$ Bennett et al. 1954, p. 306: "[T] he magnitude of $P$ [chance proportion of agreement] is dependent upon the actual number $[k]$ of response categories...' (e.g., decimal places), noting that $1 / k$ is $P$ 's chance estimate; cf. Krippendorff $2004 \mathrm{~b}$, p. 417. Butcher et al. 2008, p. 80, allude to the artificiality of comparing scales with different precision $_{1}$, although they seem, oddly, to excuse this as a benefit of ASPECTS.

${ }^{244}$ Krippendorff 2004a, pp. 217-8, warns against this methodological pitfall in reliability assessment. 


\section{A Case in Point}

In this clinically ominous case, ASPECTS misrepresented volume and eventually was ignored. A patient, aged 34 , at transfer $\approx 15$ hours after onset of global aphasia and right hemiplegia, had a dense MCA sign (Fig. 7.5), NIHSS $=24,{ }^{245}$ and an infarct rated as ASPECTS 3 (sparing M4-6). Thrombectomy was denied. However, CTP showed a $21 \mathrm{~cm}^{3}$ core in $89 \mathrm{~cm}^{3}$ of ischemia (Fig. 7.6). This fit the patient into the DAWN and DEFUSE 3 inclusion criteria except for DEFUSE 3's online CT-ASPECTS exclusion for cases recruited by CTP without MRI when late reperfusion can shrink CBF's apparent core ${ }^{246}$ (explained in note ${ }^{284}$ ). The contradictory CT and CTP led to an MRI, whose core was estimated as "50-60 ml” (method not stated). Thrombectomy was performed. (continued)

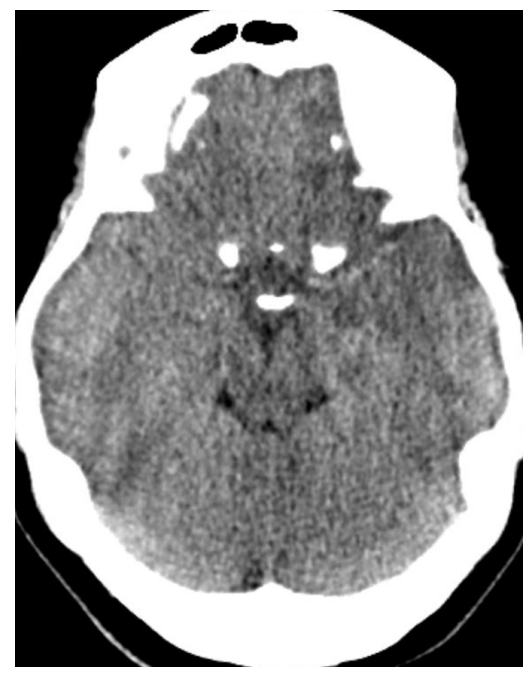

Fig. 7.5 (left). Plain CT post-transfer shows a dense MCA. (The left medial orbitofrontal lucency was artifactual.)

Fig. 7.6. RAPID $^{\circledR}-\mathrm{CTP} \approx 15$ hours after "last known well" shows: $A=21 \mathrm{~cm}^{3}$ core by $\mathrm{CBF}<30 \%$. $B=89 \mathrm{~cm}^{3}$ total ischemic zone by $\mathrm{T}_{\max }>6.0 \mathrm{sec}$.

The mismatch volume is $68 \mathrm{~cm}^{3}$. The mismatch ratio $(B / A)$ is 4.2 .

Even this small core in $A$ scores caudate, lentiform, and insular points and at least $\mathrm{M} 1$ and $\mathrm{M} 4$ in the corona radiata. Therefore by the official definitions in §3, CBF-ASPECTS $\leq 5$, a red herring.

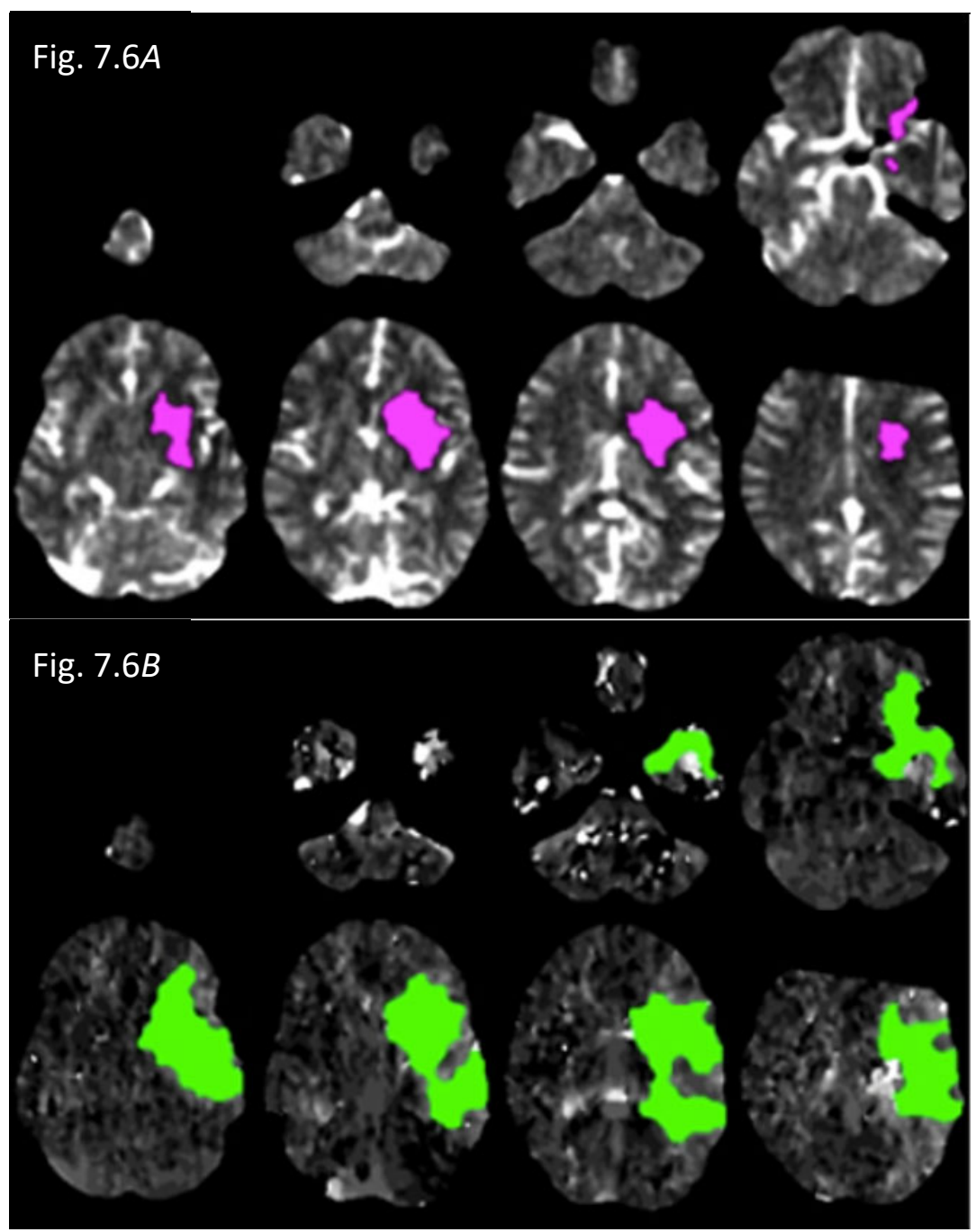

245 National Institutes of Health Stroke Scale - the higher the score, up to 42, the worse (\$8).

${ }^{246}$ DAWN: Nogueira et al. 2018a, p. 12; DEFUSE 3: Albers et al. 2018, pp. 708-9 and online 2017 Protocol p. 8 (\$9). 

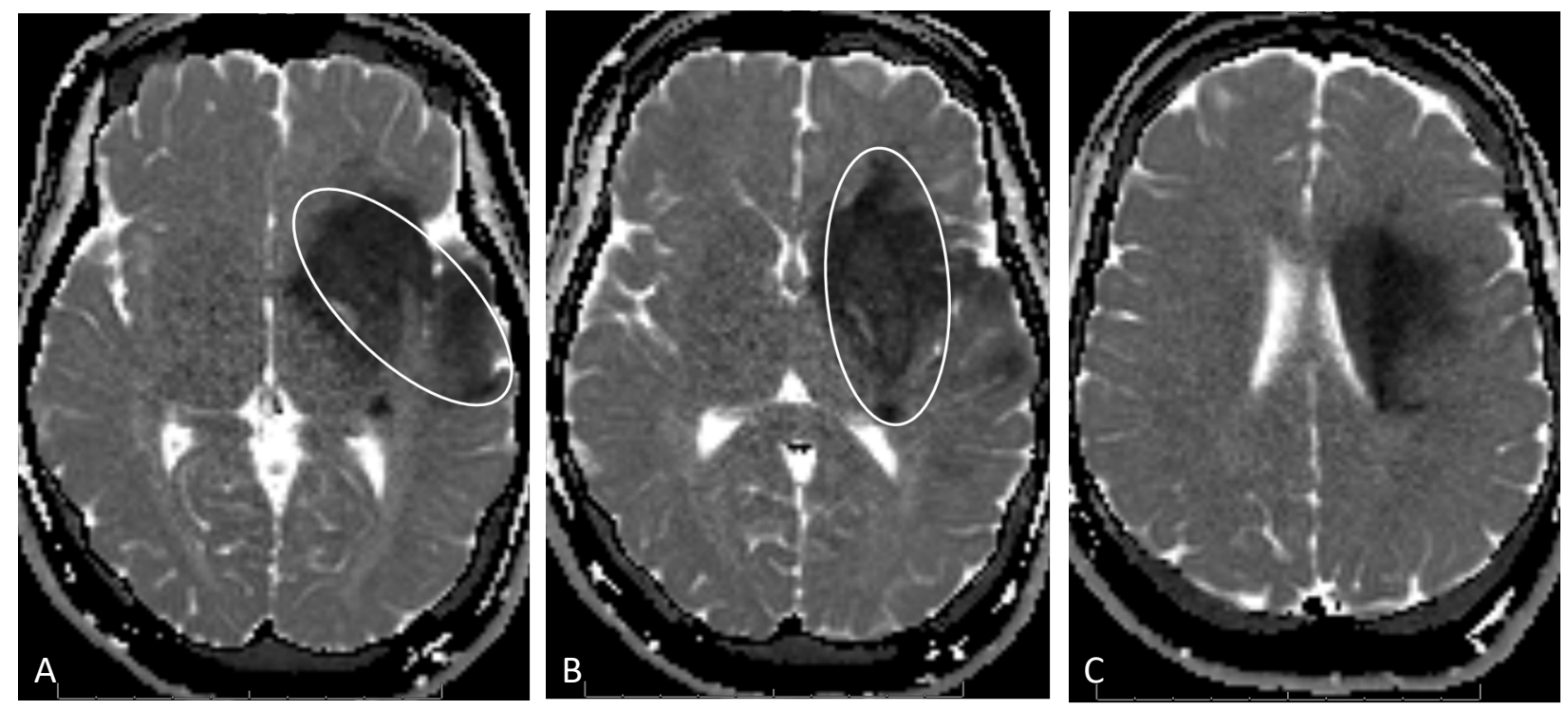

Fig. 7.7. Left MCA infarct, imaged before reperfusion as an ADC (apparent diffusion coefficient) map, showing the infarct's cytotoxic edema as darkness. Blurred edges, not explained by volume averaging as a section-thickness artifact, suggest, on the "continuum" of completeness, "incomplete infarction."247 Such blurring and incompleteness might influence how edges are pinpointed to measure extent.

The MRI (Fig. 7.7) illustrates the use of $2 S h / 3$ and the discrepancy between it and ASPECTS. The ellipse was aligned to the long axis in $\mathrm{B}$ and adjusted to an iso-area estimate $(S)$. Transferred to $\mathrm{A}$ and rotated, it remains a good iso-area approximation even while taking liberty with the long axis. Its area by $\pi r_{1} r_{2}$ is $18.6 \mathrm{~cm}^{2}$. The affected sections span $5.5 \mathrm{~cm}(h)$ by their center positions (which allow for partial thickness end-section involvement), yielding $68 \mathrm{~cm}^{3}$ (no relation to the CTP mismatch's apparent penumbra). Besides ASPECTS's four small plus M1-3 regions (already counted by CT), the second-highest affected section, C, involves M4-6 (\$3.2 refutes the cortex-only interpretation), reaching ASPECTS 0. One might quibble over M6 or the exact volume, but as a reasonable best estimate, $68 \mathrm{~cm}^{3}$ or anything near it is a clinically significant correction from ASPECTS $\leq 3$.

Because the CT extent was so much greater than the $\mathrm{CBF}$ core, probably the latter underestimates the true core that the ADC map shows more reliably (rather than the ADC reflecting infarct growth). Late leptomeningeal reperfusion might justify a CT volume exclusion, but not CT-ASPECTS. After thrombectomy, aphasia improved to expressive, strength improved somewhat, and NIHSS $=16$. Despite the residual deficits, the personal value of these improvements is clinically significant. This patient narrowly escaped the toxicity of ASPECTS.

We deal here not with legalistic rigid formalities but with a clinical style of judgment in an estimation step for $A B C / 2$ and $2 S h / 3$ that is far simpler than some other accepted tasks in stroke care. The barrier is unfamiliarity, cured by a little practice for those who don't take to it intuitively.

247 Garcia et al. 1996. 
Page 60 ASPECTS, The Mismeasure of Stroke: A Metrological Investigation

\section{§8. ASPECTS Repurposed}

If ASPECTS's validity (\$2.3) for volume is so low, can it be passed off as an alternative to volume? Barber et al. mentioned "functional outcome and the risk of symptomatic haemorrhage" (\$3.1 at (5)), and one of the authors followed with "to predict not only those who are at a lower risk of haemorrhage but also to select patients who are more likely to benefit from the intervention."248 If the latter sounds like another way to say "functional outcome," it isn't exactly, because even a patient anticipated to have a poor functional outcome can have a usefully less poor outcome with effective treatment (e.g., in §7). Infarct volume could relate in different ways to sICH risk, functional outcome, and treatment effect, the latter two, at least, modified (perhaps differently) by infarct distribution. Getting past raw volume (whether core or penumbra) to the complexities of an anatomically nuanced prognostication or therapy criterion is no arbitrary matter. It would require deliberation and clinical correlation.

In 2005 some ASPECTS advocates tried to move its goalposts from the volume-denominated risk of hemorrhage, and its surrogate the $1 / 3 \mathrm{MCA}$ rule, to a supposed functional weighting of tissue.

ASPECTS provides a reliable semiquantitative, localization-weighted estimation of ischemic tissue volume within the MCA territory, which correlates with functional outcome after rtPA.

That passage and its citations ${ }^{249}$ give no rationale for, or explanation of, "localization-weighted."

The ASPECTS is ... a topographic scoring system, .... Areas of the MCA territory are weighted based on functional importance (localization weighted) rather than extent, with equal weighting given to smaller structures, such as the internal capsule, basal ganglia, and caudate, as is given to larger structures, such as the posterior temporal (M3) and inferior frontal (M1) lobes. ${ }^{250}$

This explicit change of mission from "extent" to "functional importance" cites no one. The articles give no rationale for this change, or any methodology for how to weigh functional importance. ASPECTS would turn from what CT does, toward what neurologists do better (on which, more below).

The ASPECTS is a semiquantitative grading score that subdivides the MCA territory into 10 regions of interest combining information about edema volume and localization.

But this article, “... Prognostic Value of the Alberta Stroke Program Early CT Score in ECASS II,” says "In ECASS II, the effect of rt-PA on functional outcome is not influenced by baseline ASPECTS. Patients with low ASPECTS have a substantially increased risk of thrombolytic-related PH." findings return ASPECTS, from function and treatment effect, to hemorrhage risk and plain volume, e.g., "ASPECT Scoring to Estimate >1/3 Middle Cerebral Artery Territory Infarction.,"252

248 Buchan 2001, p. 99, citing Barber et al. 2000. Cf. Nogueira \& Ribó 2019, p. 2615: “stroke treatment selection paradigms should be based on treatment effect modifiers rather than predictors of outcomes."

249 Demchuk et al. 2005, p. 2113 (quoted), citing Barber et al. 2000, Pexman et al. 2001, and Coutts et al. 2004. The last, not mentioning outcomes, may have been numbered in error for the next-numbered Barber et al. 2005 (then in press), also not mentioning outcomes but at least mentioning "topographical" (p. 1529) although not mentioning weighting.

${ }^{250}$ Demchuk \& Coutts 2005, p. 410; cf. Menon et al. 2011, p. 408: repeating "weighted based on functional importance"; and cf. Parsons et al. 2005, p. 678. The reiterations have no scientific support from cited references.

${ }^{251}$ Dzialowski et al. 2006, pp. 973-4. Cf. Hill et al. 2014, p. 446: "clinical stroke severity, vessel occlusion, location, and ASPECTS are correlated variables," "The treatment effect was not modified by the dichotomized ASPECTS."

${ }^{252}$ Demaerschalk et al. 2006, title. 
Yet some still claim ASPECTS is "a strong predictor of functional outcome" with "modification" on "the effectiveness of intraarterial thrombolysis"; then still citing "volume estimation" in line with the $1 / 3$ MCA rule, they call ASPECTS "a weighted volumetric scale" - "based on functional importance."253 They give no citation, rationale, or methodology to support ASPECTS’s 7-8 $\times$ small-region bias $(\$ 6.1)$ or generally for biasing the natural variable of tissue volume by a convenience sample of weights.

Suppose we abandon infarct volume measure for hemorrhage risk, and shift gears to weighting for neurological function and probable clinical outcome from a structure's unilateral infarction. The internal capsule is a strategic choke point for motor function that may deserve to be overweighted (whether its segments have ACA, MCA, AchA, or PCA perfusion, §4), if "Acute [corticospinal tract] damage at the level of the PLIC is a significant predictor of unfavorable motor outcome."254 But by how much should it be overweighted? Even if we guess $7-8 \times$, it might be the only region so deserving. The caudate's function is more arcane, something like "to control approach-attachment behavior.",255 A review of 240 cases of basal ganglia lesions (caudate, putamen, globus pallidus), while finding movement and behavioral disorders, noted "the inevitable tendency to report only those cases with specific deficits associated with lesions in brain structures of interest. The multitude of cases with basal ganglia lesions without such deficits encountered in routine clinical practice go unreported"and more emphatically, "for every case with an overt behavioural and movement disorder caused by a lesion in striatum or globus pallidus, there are many more cases encountered in the clinic in which similar lesions have no such effects." 256 Extensive isolated unilateral capsuloganglionic infarction has been seen with excellent outcome. ${ }^{257}$ Since ASPECTS scores any involvement of a region (\$3.2), often little long-term morbidity could be expected. The caudate is even more overweighted than indicated in \$6.1 because, of the "two levels" defined by the caudate head's presence in the lower, the caudate body along M4-6 in the higher level affords an extra chance to score its point. ${ }^{258}$ Although the insula is "a limbic integration cortex" which "contributes to multiple functions critical for human cognition and behavior," ${ }^{259}$ patients with infarcts isolated to it usually do well. Of 15 with known outcomes, at 6 months the modified Rankin Scale (mRS) was 0 in 8 and 1-2 in $7 .{ }^{260}$ Insular involvement, at least by " $>50 \%$ " and perhaps just "right sided," may increase mortality, ${ }^{261}$ but to a considerable degree the insula gets a bum rap from the large-infarct company it tends to keep. ${ }^{262}$

\footnotetext{
${ }^{253}$ Puetz et al. 2009, p. 355, emphases added; see their quotation in §6.1. Cf. Butcher et al. 2008, p. 80.

${ }^{254}$ Puig et al. 2011 (quoting from abstract).

255 Villablanca 2010, p. 95 (including matters of drive, cognition, perseveration, hyperactivity, posture, and accuracy). More generally in the basal ganglia Thiebault et al. 2021 find "a supramodal syntactic function" for language and tools.

${ }^{256}$ Bhatia \& Marsden 1994 (quoting pp. 867, 871); cf. Campbell et al. 2016, p. 19: "Three points can be lost for caudate, lentiform and insula involvement (which in isolation will generally be minimally clinically symptomatic)."

257 personal communications from neurointerventional colleagues J.B. and M.A.; cf. Ospel, et al. 2021b.

${ }^{258}$ Puetz et al. 2009, p. 355; Menon et al. 2011, p. 408; cf. Kuang et al. 2019, p. 34, Fig. 1; but cf. \$3.2's comment "that the M5 ... extends medially to the margins of the lateral ventricle," which on this point seems superseded or forgotten.

259 respectively: Augustine 1996; Uddin et al. 2017, p. 304.

${ }^{260}$ Lemieux et al. 2012, p. 84; the infarcts were anterior or posterior insula or both, and were on either side.

261 Timpone et al. 2015; Christensen et al. 2005 (quoted respectively).

${ }^{262}$ Fink et al. 2005; Wu et al. 2015; Payabvash et al. 2017, esp.p. 62; Seyedsaadat et al. 2021, pp. 1106-7. These, and ${ }^{260}$, seem to be contradicted by, or to contradict, Payabvash et al. 2018 on the insula as an independent adverse predictor.
} 
Like volume, function is probably best measured directly — here, clinically. The National Institutes of Health Stroke Scale (NIHSS) examines 11 clinical domains with 15 tests scoring deficits up to 42 points. It requires 10 minutes to administer using simple materials distributed free, ${ }^{263}$ is "a valid tool to assess stroke severity" and "a strong predictor of outcomes after stroke," and has been measured to be a better outcome predictor than ASPECTS. ${ }^{264}$ "Prediction models such as the iScore and the ASTRAL include the NIHSS as one of several predictors," and do not mention ASPECTS ${ }^{265}$

The NIHSS reflects laterality. After a decade it was reported that a given score tends to correspond to a larger infarct on the right than the left. This was considered a defect ${ }^{266}$ until it was realized that

despite comparable incidence of acute left and right lesions, lesions primarily in the left middle cerebral artery territory were associated with poor mRS. ... [I]njury to the left hemisphere ..., to the motor pathway (ie, posterior limb of the [IC], corona radiata) and white matter tracts, [was] associated with greater severity of acute stroke symptoms and poor long-term outcome. ${ }^{267}$

This is expected from language and right upper extremity control in some left " $\mathrm{M}$ " regions (\$3).

Apparent disproportionate significance of caudate, lentiform, and insular involvement may arise in part from a greater tendency to be involved in large infarcts. In the right hemisphere, at least:

Once volume is included in the models, lesion location in the right hemisphere is no longer significant for either NIHSS or mRS. ... We speculate that the reason ... is that the size of the lesion in right hemispheric strokes determines the degree of admission stroke severity and outcome, independent of where the large lesion is located in the right hemisphere.

Thus emphasized is "the importance of accounting for the acute infarct volume in prediction models":

[S]tudies of brain topology and outcomes that do not account for lesion size may mistakenly attribute disproportionate significance of injury to regions that tend to be associated with large lesion volumes ... these regions may not be independently associated with poor outcome. ${ }^{268}$

263 developed by Brott et al. 1989; named and tested for interrater agreement by Goldstein et al. 1989 using weighted $\boldsymbol{\kappa}$; for details see https://www.stroke.nih.gov/documents/NIH_Stroke_Scale 508C.pdf. Also available are versions shortened to 11 items (mNIHSS: Lyden et al. 2001, Meyer et al. 2002) and 8 or 5 items (sNIHSS-8, sNIHSS-5: Tirschwell 2002). Other stroke scales are the Canadian Neurological Scale, the Mathew Scale, Ogogorzo Scale, and the Scandinavian Stroke Scale, and then there are disability, handicap (e.g., mRS), and quality of life scales (De Haan et al. 1993).

${ }^{264}$ Muir et al. 1996; Faigle et al. 2014; Kwah \& Diong 2014 (quoted). ASPECTS “discriminated individual outcomes weakly" (Weir et al. 2006, p. 516, cf. Table 2, p. 518) and did not independently predict poor outcome (González et al. 2012, p. 1; Marnat et al. 2019, p. 1011, Table 3, and On-line Appendix p. E3, Table 3).

${ }^{265}$ Kwah \& Diong 2014 (quoted); Saposnik et al. 2011 (iScore); Ntaios et al. 2012 (ASTRAL). An unnamed 11-parameter therapy selection system by Venema et al. 2017 uses ASPECTS, where it could be a suboptimal surrogate for volume.

${ }^{266}$ Woo et al. 1999; Lyden et al. 2004; Millis et al. 2007. Sometimes no difference is found, e.g., Payabvash et al. 2017, p. 61.

${ }^{267}$ Wu et al. 2015, p. 2441; p. 2442: "injury to the corticospinal tract in the left hemisphere was associated with poor outcomes"; cf. Laredo et al. 2018: a somewhat different list (\& no relation to ASPECTS) with variable nullification by volume control; cf. Ernst et al. 2018, e.g., p. 1992: "the left deep periventricular white matter and adjacent internal capsule" (poor outcome); cf. Kawano et al. 2010: corona radiata "DWI+W" lesions militate against "early dramatic improvement"; and cf. Schaefer et al. 2015. However, Hungerford et al. 2017 find little cortical-subcortical difference and just a trend $(p=0.07)$ in subcortical infarcts for 90-day mRS to be L $>$ R, and Almekhlafi et al. 2019 find 24-hr NIHSS L $>$ R but 90-day mRS L=R.

${ }^{268}$ quoted throughout this paragraph: Wu et al. 2015, pp. 2441,2442 (emph. added); cf. a good analysis by Ospel et al. 2021c. 
"The Real Estate Factor" is the truism that "the topography of acute stroke lesions ... may provide further information with regard to presenting stroke severity and long-term functional outcomes" 269 but which topography? One proposed list of correlates for more severe NIHSS and mRS scores is

injury to white matter (corona radiata, internal and external capsules, superior longitudinal fasciculus, and uncinate fasciculus), postcentral gyrus, putamen, and operculum ... as well as the amygdala, caudate, pallidum, inferior frontal gyrus, insula, and precentral gyrus, ${ }^{270}$ albeit perhaps via large-volume infarction. To help assess a core infarct when clinical scores are expanded by penumbra, a synthesis of this and other lists might find a role, but they are differently structured, more detailed, and better founded than ASPECTS's feeble attempt $(\$ 3, \S 6.1)$.

Even if ASPECTS's crude Real Estate Factor sometimes helps its outcome correlation, ${ }^{271}$ this is a non-optimized accident of a poor measurement practice. ASPECTS's predictive correlations do not validate a special role for its special mix, but affirm only an expected correlation analogous to that between ASPECTS (inversely) and $\mathrm{sICH}(\$ 4)$. It is better science to track volume and function separately as two biological variables than to encroach on topographic research by blending and double dipping. If topographic weighting is to be attempted for outcome prediction, it should be developed by explicit investigation, ${ }^{272}$ not just grandfathered from a popular ill-founded scale.

The key measure is of what can be saved - a "penumbra" between thresholds of electrical failure (structure intact) and ion pump failure (structure condemned) approximated as a volume difference between the hypoperfusion and core regions from a CT or MRI technique. ${ }^{273}$ Hypoperfusion and core must be measured on volume continua ${ }^{274}$ — unencumbered by ASPECTS's arbitrary, diverse, large steps that exaggerate subtraction errors either way in different patients. The latter effect shows up in scatter plots of MRI-ASPECTS versus volume, comparing PWI-DWI mismatch against PWI. ${ }^{275}$ Appropriately, the AutoMIStar software "calculates various perfusion- and diffusion-related maps together with threshold maps and lesion volumes ..." without using or offering ASPECTS.$^{276}$

Repurposing cannot save ASPECTS. Its "functional weighting" detour is at a dead end.

\footnotetext{
${ }^{269}$ quoted respectively: Menezes et al. 2007 (title, cf. Saver et al. 1999, p. 296: “lesion location”); Wu et al. 2015 , p. 2438.

270 Wu et al. 2015, p. 2438. Others: Phan et al. 2010, Cheng et al. 2014; Ernst et al. 2017, p. 2428 (Fig. 1); Ernst et al. 2018, E2-3; Laredo et al. 2018; cf. Sillanpää et al. 2011, p. 15: "strategic infarcts that have a relatively small volume but ... considerable impact ...." Cf. the IST-3 scale (\$6.2). Payabvash et al. 2017 (p. 59) suggest “development of a 'hazard atlas' of the brain to predict clinical outcome"- evidently not considering ASPECTS to be (based on) one.

271 Olivé-Gadea et al. 2019; but cf. contrary literature in \$4. Either way, "its role in determining which patients are likely to benefit from endovascular therapy (EVT) has not been well defined"-ibid., p. 198. See remark on penumbra, below.

${ }^{272}$ Yoo et al. 2012, p. 1329: "additional research ... is necessary before lesion topography can be used to ... predict outcome in the clinical setting. ... functional connectivity ...”; Nael et al. 2019, p. 1709: "a fertile area for future research.”

273 coinage: Astrup et al. 1981, p. 724, continuing Opitz \& Schneider's (1950) dual-threshold model—cf. Hossmann 1994; measurement, e.g.: Davis \& Donnan 2014, Gomez 2018.

274 e.g.: Asdaghi et al. 2014, p. 661; Leslie-Mazwi et al. 2019, p. 619; Byrne et al. 2019, p. 8. "mismatch ... calculations depend on accurate core measurement" (jettisoning, when the chips are down, the ASPECTS that their algorithm starts with).

275 Lassalle 2016, p. 2556. DWI scatter (not shown) probably $\approx$ PWI scatter, but ASPECTS mismatch is much worse.

276 Apollo Medical Imaging Technology 2019—no mention of ASPECTS. (No product endorsements are intended.)
} 
Page 64 ASPECTS, The Mismeasure of Stroke: A Metrological Investigation

\section{§9. Whither ASPECTS?}

For intravenous use of tPA, any volume or ASPECTS criterion has become largely moot. Volume criteria still are considered in research on tPA use past 4.5 hours post-onset. ${ }^{277}$ But for conventional tPA use within 4.5 hours post-onset, the AHA/ASA 2017 Scientific Statement on “... Intravenous Alteplase in Acute Ischemic Stroke ..." makes no mention of the $1 / 3$ MCA rule, any volume criterion, or ASPECTS. It lists seven "scoring systems integrating multiple factors" 278 (omnibus indexes, §6.2, which ASPECTS aspires to exemplify, §8), without mentioning ASPECTS, and concludes that "the upper range of absolute sICH risk predicted by the scores does not justify withholding thrombolytic therapy."279 A Cochrane Systematic Review "did not find any evidence of an interaction between visible infarct extent and rt-PA on death or dependency."280 The 3035-patient IST-3 trial concludes that "The clinical effect of r-tPA in patients at a higher predicted risk of sICH or poor functional outcome was at least as good as, and possibly more than, in patients with a lower risk.",281 Finally, the AHA/ASA 2018 Guidelines leave ASPECTS behind:

3. There remains insufficient evidence to identify a threshold of acute CT hypoattenuation severity or extent that affects treatment response to IV [intravenous] alteplase. The extent and severity of acute hypoattenuation or early ischemic changes should not be used as a criterion to withhold therapy for such patients who otherwise qualify."282

The original clinical pretext for ASPECTS has virtually disappeared. If ASPECTS is to survive, it must look elsewhere for a cause.

Mechanical thrombectomy (MT) for large vessel occlusion (LVO) may have roles in the ACA and posterior circulations, ${ }^{283}$ but its use and investigation are concentrated on the MCAASPECTS's previously arbitrarily chosen territory (\$3.1). With improved equipment and expertise, MT trial results turned favorable in reports from 2015 onward. Unfortunately some of the trials used the biased and noisy ASPECTS to enroll patients, and most used it to analyze subgroups (Table 9.1). Some clinicians and researchers may therefore persist in using ASPECTS based on precedentmerely an administrative precedent of no scientific merit in the face of contrary evidence and logic. But even this precedent gives more of a nod to volume than to ASPECTS for patient selection.

277 e.g., Davis et al. 2008 (1/3 MCA, p. 300); Thomalla et al. 2018 (1/3 MCA, p. 613); Schwamm et al. 2018 (100 cm³, p. 982); Ma et al. 2019 (70 $\mathrm{cm}^{3}$, p. 1796); Ringleb et al. $2019\left(1 / 3 \mathrm{MCA}\right.$ or $100 \mathrm{~cm}^{3}$, p. 485).

278 Yaghi et al. 2017, quoted respectively: p. e343 (i.e., for “within 4.5 hours from onset”) and pp. e346-7. In comparison, Nishi et al. 2019, p. 2380, include two more systems (for large vessel occlusion: PRE, HIAT2) that include SPECTS.

279 Yaghi et al. 2017, pp. e346 (quoted), e347 (Table 3). Cf. Saver 2007, p. 2282: "highly favorable benefit-risk ratio."

280 Wardlaw et al. 2014/2016, p. 21

${ }^{281}$ Whiteley et al. 2014, p. 1005, cf. p. 1000: "clinicians may be unduly concerned about the early risk of sICH"; cf. Wardlaw et al. 2015, p. 493: "the extent of early ischaemic signs on CT per se should not deter treatment with alteplase"; IST-3 Collaborative Group 2015: "No interaction was recorded between any individual or combined imaging variable and alteplase, for either functional outcome ... or symptomatic intracranial haemorrhage" (p. 492), "All data combined show that the extent of early ischaemic signs on CT per se should not deter treatment with alteplase" (p. 493, box).

282 Powers et al. 2018, p. e58. For history and references: Lyden 2019, particularly p. 2599. Powers et al. 2019, p. e369, maintains this guideline but abbreviates the statement.

283 ACA: Uno et al. 2018 (some primary, some $2^{\circ}$ to MCA MT); Post. Circ.: Fahed et al. 2018b, p. 3; Baik et al. 2019. 
Among the 12 published major prospective randomized trials of MT versus no MT that did not just exclude all CT-visible infarcts, for patient inclusion and exclusion criteria by infarct size (Table 9.1):

3 had no ASPECTS or volume criterion (MR RESCUE, MR CLEAN, THRACE),

1 used ASPECTS only and not volume (REVASCAT),

4 used ASPECTS and (or) volume (IMS III, ESCAPE, SWIFT PRIME, DEFUSE $3^{284}$ ),

4 used volume only and not ASPECTS (EXTEND-IA, THERAPY, PISTE, DAWN).

More of these trials enrolled by volume without ASPECTS than by ASPECTS without volume. ${ }^{285}$ Hardly a collective endorsement for ASPECTS, the precedent here is more for using volume.

Trial subgroup analysis is another matter: 9 reports used ASPECTS at one or two arbitrary cuts. Still, 3 (MR RESCUE, EXTEND-IA, DAWN) spurned ASPECTS. Investigators from the five 2015 trials, all favorable to thrombectomy, pooled their data in a patient-level meta-analysis, HERMES, and for subgroup analysis used two arbitrary ASPECTS cuts at $9 / / 8$ and 6//5. ${ }^{286}$ No trial compared ASPECTS to volume for precision and accuracy (cf. Table 4.1).

The limited subgroup analyses were prematurely "confirmatory," rather than "exploratory" in search of the shape of any therapeutic effect curve ${ }^{287}$ Either way they were methodologically lax to use such a biased and noisy measure as ASPECTS (\$6) at all, and further in checking just one or two arbitrarily chosen ASPECTS thresholds. Investigators may need the binning effect of pooled scores in order to accumulate testable statistics, but that is another symptom of a rush to confirmation after insufficient exploration. Even given the use of ASPECTS, the trials could have investigated all cuts systematically in a reasonable range, say $8 / / 7$ to $4 / / 3$, to get a sense of the effect curve or best cut $(\S 4)$, and HERMES could have investigated them each too. Only tests of alternative dichotomizations can suggest one particular threshold is preferable to others. This is calibration, not $p$-hacking, though it would then require appropriately cautious confirmatory statistical testing to compare thresholds. "Randomized clinical trials specifically designed to address the limits where thrombectomy is no longer beneficial are needed." These trials would benefit from true infarct volume measurement, because "If useful models produce better science, then what drives better models? Improved measurements. ... If we can't reliably measure something, it's hard to build a theory about it."288 Better measurement may find no natural threshold but will focus the effect curve more reliably and serve opinion better.

${ }^{284}$ Albers et al. 2018: for $70 \mathrm{~cm}^{3}$, pp. 708-9; Clinical Protocol Version 2.4 (Apr. 2017, p. 8) adds an exclusion criterion of "ASPECT score $<6$ on non-contrast CT (if patient is enrolled based on CT perfusion criteria)." So while "For DWI cases ASPECTS was never used as an exclusion," CT-ASPECTS [ $\leq 5]$ was potentially an exclusion among patients enrolled by CTP, where by $\approx 16$ hours leptomeningeal reperfusion can begin to mask the core, but "This happened only on a few occasions"-Albers 2019 (both quotes + included paraphrase). PWI, with DWI, is immune to this problem (cf. §6.3). DEFUSE 3, as printed, is often cited as enrolling by $<70 \mathrm{~cm}^{3}$ without ASPECTS (Schellinger \& Demaerschalk 2018, p. 2560; Powers et al. 2018, Supp. 1, p. 41; Leslie-Mazwi et al. 2019, pp.618-9; Turc et al. 2019, p. 5; Nael et al. 2019, p. 1698.)

285 And for just comparing 2 MT methods - of 3 such trials listed in Powers et al. 2019 (Supp. 1, Table XVII, pp. 35-48), ASTER (Lapergue et al. 2017) and Penumbra Separator 3D (Nogueira et al. 2018b) also did not select patients by ASPECTS.

${ }^{286}$ Goyal et al. 2016a, p. 1728, Figure 2. Outside of quotes we use $\$ 4$ 's “//" notation to avoid the confusing $>, \geq, \leq,<$.

${ }^{287}$ quoted terminology from, e.g., Hartwig \& Dearing 1979, p. 10.

288 quoted respectively: Fisher \& Goyal 2018, p. 1806; Smaldino 2019, p. 9 (italics added). 
Table 9.1. Published Prospective Randomized Controlled Trials of MT Versus No MT.

\begin{tabular}{|c|c|c|c|c|}
\hline CLINICAL TRIALS & Patients & Volume(-like) Exclusion Criterior & \& C.A.* & Subgroups \\
\hline IMS III' 289 & 656 & $>1 / 3 \mathrm{MCA}$ (V $5 / / 4 \mathbf{t}^{+}$"as a guideline") & $A \vee$ vol & $8 / / 7$ \\
\hline SYNTHESIS Exp'n 290 & 362 & Visible acute infarct (CT) & moot & ( $\varnothing$ ASPECTS) \\
\hline MR RESCUE 291 & 118 & No volume exclusion & neither & ( $\varnothing$ ASPECTS) \\
\hline MR CLEAN ${ }^{292}$ & 500 & No volume exclusion & neither & $8 / / 7 \& 5 / / 4$ \\
\hline EXTEND-IA 293 & 70 & $>1 / 3 \mathrm{MCA}$ (CT) $\vee>70 \mathrm{ml}$ (CTP) & vol & ( $\varnothing$ ASPECTS) \\
\hline ESCAPE $^{294}$ & 316 & $6 / / 5 \vee \frac{1}{3} \mathrm{MCA}$ (CTP) & $A \vee$ vol & $8 / / 7$ \\
\hline SWIFT PRIME ${ }^{295}$ & 196 & $6 / / 5$ (CT V DWI) $\vee 1 / 3 M C A$ & $A \vee$ vol & $8 / / 7$ \\
\hline REVASCAT $^{296}$ & 206 & $7 / / 6$ (CT) $\vee 6 / / 5$ (DWI) & A & $8 / / 7$ \\
\hline THRACE $^{297}$ & 414 & No volume exclusion & neither & $8 / / 7 \& 5 / / 4$ \\
\hline THERAPY ${ }^{298}$ & 108 & $>1 / 3 \mathrm{MCA}$ & vol & $8 / / 7 \& 5 / / 4$ \\
\hline PISTE $^{299}$ & 65 & $>1 / 3 \mathrm{MCA}$ & vol & $8 / / 7 \& 5 / / 4$ \\
\hline DAWN $^{300}$ & 206 & $\mathrm{~A}, \geq 21 \mathrm{ml} ; \mathrm{B}, \geq 31 \mathrm{ml} ; \mathrm{C}, \geq 51 \mathrm{ml}$ & vol & ( $\varnothing$ ASPECTS) \\
\hline DEFUSE $3^{301}$ & 182 & $\geq 70 \mathrm{ml} \vee 6 / / 5$ (CT) & $A \vee$ vol & $8 / / 7$ \\
\hline
\end{tabular}

BOLDFACE: The 8 trials that yielded positive results favoring MT (mechanical thrombectomy). *C.A. = A content analysis (https://en.wikipedia.org/wiki/Content analysis, \$10) categorization for the type of exclusion criterion: $A=A S P E C T S$, vol = volume, $V=$ or. $\dagger_{x} / / y$ dichotomizes ASPECTS as $\geq x$ versus $\leq y$.

289 Broderick et al. 2013: for 5//4, Protocol Amendment \#1, March 13, 2006, and Protocol June 27, 2011, p. 33; for $8 / / 7$, Protocol Dec. 28, 2005, p. 50, \& June 27, 2011, p. 66; Supp. pp. 6, 30 says " $<4$ " as $4 / / 3$ or perhaps in error. 290 Ciccone et al. 2013 (“Expansion," equivalent to a “II"), Protocol Versions 1 \& 2, pp. 4, 11 (as indicator of $>4.5$ hours). 291 Kidwell et al. 2013.

292 Berkhemer et al. 2015, pp. 17: "no significant interaction between subgroups and treatment effect"; Yoo et al. 2016.

293 Campbell et al. 2015, 1009, Supplementary Appendix pp. 4, 5.

${ }^{294}$ Goyal et al. 2015: for 6//5, pp. 1021, 1024, Protocol p. 7; for 8//7, p. 1027, Supplement pp. 13, 15.

${ }^{295}$ Saver et al. 2015: p. 2293 for $6 / / 5$ and 8//7, and online materials passim adding $V 1 / 3 M C A$ in online Table S1, passim. 296 Jovin et al. 2015: for 7//6 \& 6//5, pp. 2297, Supp. pp. 4, 23, 26-8, Protocol pp. 4, 24, 26-8, 38; for 8//7, p. 2305

${ }^{297}$ Bracard et al. 2016: Supp. p. 2: exclusion for midline shift as sign of timing error; p. 1141 for ASPECTS subgroups. 298 Mocco et al. 2016: Supp. p. 5, “Any acute ischemic changes in >1/3 ..."; p. 2333 for ASPECTS subgroups.

299 Muir et al. 2017, pp. 39-40.

${ }^{300}$ Nogueira et al. 2018a, p. 12, age groups: $A \geq 80$ \& NIHSS $>10 ; B<80$ \& NIHSS $\geq 10 ; C<80$ \& NIHSS $\geq 20$ \& vol 31-50.

${ }^{301}$ Albers et al. 2018: for 70 ml, pp. 708-9; for 6//5, Protocol 2017, p. 8; for 8//7, p. 716, Statistical Analysis Plan 12.2. 
The AHA/ASA 2018 and 2019 Guidelines endorse the HERMES $6 / / 5$ cut electively for $\leq 6$ hours:

3.7.2. 1. Patients should receive mechanical thrombectomy with a stent retriever if they meet all the following criteria: ... (5) ASPECTS of $\geq 6$; ... [It does not restrict by "only if" or "iff"]

4. Although its benefits are uncertain, the use of mechanical thrombectomy with stent retrievers may be reasonable for patients with AIS [acute ischemic stroke] in whom treatment can be initiated (groin puncture) within 6 hours of symptom onset and who have ... ASPECTS $<6, \ldots .{ }^{302}$

There is no real ASPECTS exclusion at $<6$ hours. Another guideline does not mention ASPECTS-

2.2.4. 1. When selecting patients with AIS within 6 to 24 hours of last known normal ..., obtaining CTP or DW-MRI ... is recommended to aid in patient selection for mechanical thrombectomy, but only when patients meet other eligibility criteria from one of the RCTs that showed benefit from mechanical thrombectomy in this extended time window.

- except indirectly by reference to the DEFUSE 3 trial, which makes little use of ASPECTS ${ }^{\mathbf{3 0 3}}$

Echoing the possibly greater salutary effect of intravenous thrombolysis found "in patients at a higher predicted risk of sICH or poor functional outcome" (IST-3, above), IMS III found greater improvement, with MT compared to IV tPA alone, in patients with NIHSS $(\S 8) \geq 20$ versus $\leq 19$. For $\geq 20$, mRS $0-2$ rose by $42 \%$ and $0-3$ rose by $48 \%$ with endovascular therapy, compared to negligible declines for $\leq 19$. This striking effect was considered not statistically significant and "The trial was stopped early because of futility." ${ }^{304}$ DAWN nevertheless notes this difference: "Although not statistically significant for clinical benefit, the IMS-III trial suggested that patients with NIHSS $\geq 20$ could potentially have greater benefit from [MT] as compared to those in the 8-19 category."${ }^{, 305}$ To prove that MT benefits large infarcts, imaging-based selection will play a central role, ${ }^{306}$ which will require support by a more scientific kind of volume measurement than ASPECTS.

Guideline 3.7.2. 1 is multifarious. ${ }^{307}$ It says three things at the same time, but only doing MT was randomized and controlled, so this alone earned evidence Level A and recommendation Class I. The " $\geq 6$ " and "ASPECTS" shadow ephemerally only as precedents with untested comparative merit. The high-end (volume) or low-end (ASPECTS <6) drop-off of confidence in MT benefit, as seen by HERMES in the fog of ASPECTS, does not say where or whether the stochastic benefit drops off. ${ }^{\mathbf{3 0 8}}$ The use of ASPECTS has No Level of evidence and deserves No Class of recommendation.

302 Powers et al. 2019, pp. e372-3; cf. Powers et al. 2018, p. e71, 3 and 6. For benefit at <6, see Desilles et al. 2017, Mourand et al. 2018, Goyal et al. 2018 (esp. remarks by Jovin), Kaesmacher et al. 2019b, and Turc et al. 2019 , p. 537.

303 Powers et al. 2019, p. e358, and now see p. e374, [3.7.3] 1 \& 2, referring in more detail to DAWN and DEFUSE 3, again without mentioning ASPECTS; cf. Powers et al. 2018, p. e59, 12. On ASPECTS in DEFUSE 3, see note ${ }^{\mathbf{2 8 4}}$.

304 Broderick et al. 2013, p. 898, Figure 1: rises are \%/\%, not percentage points; $\mathrm{mRS}=$ modified Rankin Scale at 90 days.

305 Nogueira et al. 2018a, Supp. Appx. p. 32; cf. Haussen et al. 2016, p. 2321: "a significant proportion of patients with CTP core $>50 \mathrm{cc}$ and ASPECTS <6 still achieved favorable outcomes, ...."

306 paraphrasing Leslie-Mazwi 2019, p. 1715.

${ }^{307}$ Nolan 1990, p. 1015: "inquir[ing] about several different facts [overall benefit of thrombectomy, whether \& where a benefit drop-off might be, and the use of ASPECTS] when each fact should be inquired about in a separate issue." On choosing the appropriate comparison to test for statistical significance, see Gelman \& Stern 2006, pp. 329-30.

308 Goyal et al. 2016a, p. 1728, Figure 2; p. 1729: "Because most trials excluded patients with an ASPECTS of 5 or lower, the effect of endovascular thrombectomy in this category of patients could not be established by our analysis." 
Until more investigators get off the ASPECTS bandwagon ${ }^{309}$ and test sharper volume thresholds (so that the Guidelines can add a confidence threshold as volume and not seem to require ASPECTS), how can we interpret the Guidelines' ASPECTS 6//5 quasi-threshold? Using §6.3's meta-analysis: ASPECTS 6 covers a range of 4-215 $\mathrm{cm}^{3}$ with a mean of $54 \mathrm{~cm}^{3}$ and a median $47 \mathrm{~cm}^{3}(n=95)$, ASPECTS 5 covers a range of $2-197 \mathrm{~cm}^{3}$ with a mean of $76 \mathrm{~cm}^{3}$ and a median $63 \mathrm{~cm}^{3}(n=69)$. While noting their ranges' paradoxical extremes, we attend to their measures of central tendency. A threshold between them can be chosen as their mean mean of $65 \mathrm{~cm}^{3}$ or mean median of $55 \mathrm{~cm}^{3}$. The mean mean is more globally representative, and it comes closer to the $70 \mathrm{~cm}^{3}(\$ 4)$ that EXTEND-IA, DEFUSE 3, and some others use. ${ }^{310}$ Indeed, DEFUSE 3 effectively (implicitly) equates ASPECTS $\geq 6$ with infarct volume $<70 \mathrm{~cm}^{3} .^{311}$ Replacing ASPECTS $6 / / 5$ with a 65 or $70 \mathrm{~cm}^{3}$ threshold would be an optimizing strategy, most closely reflecting the average of a dispersed statistic, although we do not know whether 6//5 was optimal among ASPECTS thresholds in the first place. There is no evidence for this exact guideline or for the use of ASPECTS at all in lieu of volume.

Insistence on matching ASPECTS with ASPECTS would be analogous to probability matching. ${ }^{\mathbf{3 1 2}}$ A prototype of this mistake is a paradigm of known 2:1 odds to win a prize behind Door 2 vs. Door 1 . The optimal, but boring, strategy is to choose Door 2 always. A player who matches his bets to the odds, choosing Door 2 just $2 / 3$ of the time, reduces his overall odds to win to 5:4. Another prototype is to get drunk and unsteady before shooting at a moving target. In these cases, two wrongs don't make a right. The upshot is that a $70 \mathrm{~cm}^{3}$ threshold would in fact adhere better to an ASPECTS $6 / / 5$ guideline's most probable underlying volume distribution than the more disperse ASPECTS 6//5 itself would. As perhaps a transitional compromise, the European ESO and ESMINT guidelines recommend MT if "ASPECTS $\geq 6 \ldots$ or ... core volume $\leq 70 \mathrm{~mL}$. ."313

The last potential hiding place for an ASPECTS requirement is in quasi-governmental regulations. For Comprehensive Stroke Centers, we in the United States look to The Joint Commission (TJC), which does not ordinarily take sides in scientific controversies and does not mention ASPECTS in its Comprehensive Stroke Performance Measurement Implementation Guide. Moreover, TJC affirms that "No we do not require the use of ASPECTS." 114

This report does not advocate for any particular value of an infarct volume threshold for therapy, showing just that ASPECTS is unsuited to research or express it. If "The train has left the station,"315 as if ASPECTS is locked into place, the train would need to be called back. But there is no train, and there is no logical or sound empirical basis for, or consensus on, or official mandate for ASPECTS.

\footnotetext{
${ }^{309}$ Adding to earlier examples, including Table 4.1, in a review of "Imaging Paradigms in Acute Ischemic Stroke," Menon \& Goyal 2015 treat ASPECTS as if it is the only existing volume-like infarct measure for plain CT.

310 e.g., also Byrne et al., p. 326, and cf. §4.

311 Albers et al. 2018: for $70 \mathrm{~cm}^{3}$, pp. 708-9; for $\geq 6$, Protocol 2015 \& 2017, pp. 8 .

312 e.g., Shanks et al. 2002

313 Turc et al. 2019, p. 537 (online p. 25) at PICO 8: "MT may be reasonable ... with ASPECTS $<6$ or core volume $>70 \mathrm{~mL}$."

314 Guide: Joint Commission 2015; quoted: Joint Commission 2018.

315 heard in conversation 2019-Jun-15.
} 


\title{
§10. Looking Back At and Forward From ASPECTS
}

\begin{abstract}
ASPECTS popped into view in 2000 as a set of 10 diversely sized MCA regions $(\mathbf{\$ 3 . 1}) .^{\mathbf{3 1 6}}$ Absent then and now is a research basis to justify their selection, sizes, and all-or-none scoringas opposed to, say, a whole-hemispheric partition and evenhanded scoring, or just infarct volumefor its all-but-stated purpose to support the 1/3 MCA rule (or to estimate any volume). Its regions' varied sizes and aggressive scoring show that as a volume surrogate it must admit noise and bias. Its precision $_{2}$ hardly justifies even its 1-digit precision $_{1}$ (\$2.3). Ignoring its inaccuracy (overall, and especially for many patients), ASPECTS is touted for interrater agreement and outcome correlation. Yet even "The six physicians who developed ASPECTS" and served as "six expert observers" used it discordantly. ${ }^{317}$ ASPECTS thus blends caprice into arbitrariness. If ASPECTS had been derived from evidence, this would have been cited and would have guided the designing and testing physicians uniformly. Some disagreements have been more or less resolved by custom or pronouncement (\$3.2), yet disagreements persist.
\end{abstract}

To create a scoring system that measures volume, the regions must be clear and their sizes must be chosen to be either equal in volume to make points simply additive, or else in known volume ratios so that points can be weighted accordingly. Either way, there remains the problem that block scoring misrepresents fractional involvement. A contrived remedy might be fractional points estimated at, say, quarter-point precision in the individual regions. But no such system has been developed, and none is needed because we have the simpler and truer method of volume measurement by $A B C / 2$ or, even better when feasible, $2 S h / 3(\$ 7)$ or perhaps automatic volume.

Besides the absence of a sound logical or empirical basis for ASPECTS 's peculiar composition, there is abundant evidence against it as a volume surrogate (\$6). One report shows wide dispersions of CT-ASPECTS for many infarcts, and four reports show scatter plots of volume vs. DWI-ASPECTS. The latter concur in showing marked scatter, meaning that each ASPECTS reflects a wide range of volumes. Authors from three of the DWI reports supplied their data for the present composite, which shows each ASPECTS $>0$ as corresponding to a more than 3-fold range of infarct sizes. If you don't want to take a case with a volume $>50 \mathrm{~cm}^{3}$ and you are told the ASPECTS is 3 or worse, $\$ 6.3$ suggests you are safe to reject it. But the range of volumes is 18 -fold for ASPECTS $=4$ and more than 50-fold for ASPECTS $\geq 5$, where most patients are. For each ASPECTS 5 to 8, the volumes run from $\leq 4 \mathrm{~cm}^{3}$ up to 73 (for 8 ) or else to $\approx 150-200 \mathrm{~cm}^{3}$. ASPECTS does not fix the discrepancies between CT and MRI. It is not enough that between CT and DWI, ASPECTS has a "positive relation"it still shows plenty of scatter. ${ }^{318}$ CT- and DWI-ASPECTS correlate with volume's correlations with hemorrhage and clinical outcome, but instead of settling for this extra surrogacy's poorly transitive correlation with a correlation (\$2.5), why not ask for the genuine biomarker, volume? ${ }^{319}$ The next two pages rebut some possible replies.

\footnotetext{
316 Barber et al. 2000.

317 Pexman et al. 2001 (quoting pp. 1534, 1535).

318 scatter plots: Barber et al. 2005, p. 1530 (caption quoted); Nezu et al. 2011, p. 2198; Mitomi et al. 2014, pp. 39-40.

${ }^{319}$ Kidwell \& Jahan 2015, p. 412, warn of "the risk that excluded patients may also have benefited if the correct biomarker was not chosen or optimized”- cf. final case in $§ 7$. Cf. “quantitative imaging biomarker (QIB)” in §6.3.
} 
1. ASPECTS is "simple," "straightforward," "reliable," "validated," "useful," and "helpful.,"320 Throwing adjectives at ASPECTS does not make it robust, but sounds more like a form of advertising called puffing, which is not legally binding or actionable. These claims crop up even in articles listing ASPECTS's defects. ${ }^{321}$ ASPECTS contradicts "simple" with three levels of complexity: (a) diverse understandings and implementations of what its vague definitions mean in the first place, (b) the crazy quilt hodgepodge of differently sized regions violating any sense of fair measurement, $(c)$ the all-or-none scoring that exacerbates $(b)$ and often biases scores to suggest bigger infarcts. $\S \S 5,6.2$ contradict "reliable" in its $\boldsymbol{\kappa}$ sense, and $\$ \S 6.1,6.3$ show ASPECTS to be distorted and inaccurate and thereby invalidated (\$2.3). Instead of being "useful" or "helpful" it tends to be useless or harmful. Why bother with an inaccurate surrogate variable when the primary variable is directly visible?

2. "We have nothing else." ${ }^{\text {"322 }}$ Resisting $A B C / 2$ and $2 S h / 3$ reflects either an aversion to "this 'fifth-grade arithmetic problem,', ${ }^{323}$ or the fallacy that if something is imperfect, then something more profoundly flawed is better. Medical school graduates can be expected to measure and compute at this levelwithout special training or at worst with less training than ASPECTS requires. ${ }^{324}$

\section{ASPECTS "works" (though "it's imperfect") so that "Across the years, ASPECTS has gained credence and is now used the world over .....325 We have been getting away with it because the harm tends not to be noticed. Mere correlation with sICH or functional outcome $(\$ 4)$ is a trivial claim, and correlation can be a silly game (\$2.5). Even the wide scatter in all three studies combined in $\$ 6.3$ has been called "excellent correlation." ${ }^{326}$ A correlation that is scientifically interesting for a population can be useless to treat individuals ${ }^{327}$ We can estimate human foot size from height - they correlate ${ }^{328}$ but you still must and can easily fit shoes directly. Infarct volume presents a similar duty.}

${ }^{320}$ Hill et al. 2003a, pp. 1925, 1930: "a useful clinical tool," “a straightforward portable skill, easy to learn, and easy to teach"; Coutts et al. 2004, p. e103: "a reliable clinical scale"; Demchuk \& Coutts 2005, p. 416: "prognostic tool," "useful”; Aviv et al. 2007, p. 1978: "simplicity is its strength"; Butcher et al. 2007, pp. 941,945, and Butcher et al. 2008, p. 75: "validated"; Puetz et al. 2009, p. 354ff: "simple, quick and reliable," etc.; Kosior et al. 2010, p. 456: "validated and proven to show clear benefit"; Nezu et al. 2011, p. 2196: "useful"; Wusthoff et al. 2011, p. e1555: "validated”" "reliability"; Modi et al. 2012, p. 112: "Validated to be simple and reliable"; Beslow et al. 2012, p. 2: "quick and reliable"; Schröder et al. 2014, pp.3583, 3587: "a simple and reliable/useful tool"; McTaggart et al. 2015, p.407: "straightforward"; Menon et al. 2015, p. 1454: "simple and systematic"; Han et al. 2016, p. 5: "easily applied," "widely validated"; Herweh et al. 2016, p. 439: "simple, reliable and is a strong predictor of functional outcome and ... hemorrhage ...";; Schröder \& Thomalla 2017, pp. 1,5: "useful and easily applicable tool" Pfaff et al. 2017, p. 1597: "validated, reliable"; Guo et al. 2017, p. 91: "a simple, reliable, and systematic approach"; Hampton-Till et al. 2019, p. 69: "clinically validated." More insightfully, "CT ASPECTS is superficially simple ..."-Campbell et al. 2016, p. 19.

321 puffing: Nolan et al. 1990, p. 1233; defects (e.g.): Puetz et al. 2009; Schröder et al. 2014; Schröder \& Thomalla 2017.

${ }^{322}$ heard in conversation; cf. Nogueira et al. 2018a, Supp. Appx, p. 32, that "the other existent option" is only ASPECTS.

${ }^{323}$ Huttner et al. 2006, p. 408. Cf. Decker 2018: “future proofing radiology”- surely besides “3D printing," our residents and other physicians can be "comfortable with" area-outlining, area-compensation, perpendicularity, and $+-\times / \wedge \sqrt{ }$.

324 Coutts et al. 2003: "ASPECTS Reading Requires Training and Experience" (title), calling "30-minute" too "brief." 325 quotes respectively: heard in conversation $\times 2$; Kuang et al. 2019, p. 33 .

${ }^{326}$ Lassalle et al. 2016, p. 2556, re: their references 10,11, 20 on p. 2558 which are the sources of our meta-analysis.

327 e.g., Weir et al. 2006: "the overall usefulness of ASPECTS at predicting the outcome of individual patients was low ...."; Sullivan et al. 2015, p. 822: "Correlation is a weak criterion for assessing technical performance."

328 e.g.: Giles et al. 1991, Grivas et al. 2008 (see scatter plots, p. 92), Datta Banik et al. 2016, Shankar et al. 2018. 
4. ASPECTS is sui generis, a distinct degree of freedom beyond the established workhorses of volume, delay, collaterals, swelling, hemorrhage, NIHSS, patient age, and chronic ischemic load. ASPECTS does stir the pot with some localization irregularities, but its selection of structures for overweighting poorly overlaps the significant structures listed by serious researchers cited in $§ 8$. ASPECTS is not a plausible biological entity but just an odd blend of information and misinformation.

5. ASPECTS is more fun, and game-like. Rather than exercising a pesky measurement discipline, just tally some points and be done with it. The interactive "Rate the ASPECT Score" as it was at http://www.aspectsinstroke.com/casepacs/aspects-cases in 2019 instantly tallied votes and was cute, but its very coarse trichotomy of 0-4, 5-7, 8-10 for every case was all the more uselessly imprecise. Arbitrary and esoteric, ASPECTS's aura of published authority makes it "a veritable sorcerer." 329

6. The AHA/ASA 2019 Guidelines for thrombectomy ( $\$ 9$ ) are stated in terms of ASPECTS.$^{330}$ This merely restates one facet of the problem that needs to be solved - without acknowledging $§ \S 1-9$ including the guideline in $\$ 9$ that is not stated in terms of ASPECTS. Moreover, the 2018-19 AHA/ASA Guidelines and their sources that do mention $6 / / 5$ do not scientifically validate its specific choice, do not claim it is cut and dried, and do not scientifically examine the use of ASPECTS. Medical care is not an uncritical exercise in written technicalities. Rather, the best interpretation of the Guidelines, the patient's best hope, is to go by the best bet, which is the best-bet volume for the stated ASPECTS. Despite the chaotic noise inherent in ASPECTS, average volume thresholds can be derived, as in §9. (Physicians who are used to, and thrive on, imprecision, know that all outcome measures - of sICH, $\mathrm{mRS}$, and general well-being - have their own imperfect predictability and scale imprecision.) Graduating from ASPECTS to directly measured infarct volume will advance stroke science and care by decreasing one component of imprecision. Metrology offers leadership (\$1). Metrologists do not remain frozen in the established use of old standards when more precise ones come along. ${ }^{331}$ They quite normally graduate to an improved standard by working out the least disruptive calibration. For us, too, the time has come to move on.

7. We are psychologically or administratively committed to ASPECTS. Cognitive dissonance "chang[es] the attractiveness of the alternatives" 332 - perpetuating ASPECTS adulation, suppressing $A B C / 2$ and $2 S h / 3$, and inspiring $\$ 8$ 's salvage attempt. Rational (re)consideration is needed. If ASPECTS has been written into an institution's by-laws or procedure manual, it can be written back out again. "The biggest mistake is not to admit a mistake." ${ }^{333}$

${ }^{329}$ Sullivan $1968, * 320$, originally said more generally of the outsized persuasive effect of mathematics on juries because of its seeming objectivity and usual inscrutability. Of any authority, ask whether it is authoritative or authoritarian.

e.g., "On the basis of recent guidelines, it is clear that ... ASPECTS ... will remain the mainstay ..." (Goyal et al. 2016b, p. 643), perhaps thought of as "the largest common denominator among all stroke centers"-Reidler et al. 2019, p. 451.

331 e.g.: Kirkup \& Frenkel 2006, p. 41, for voltage, replacement of "banks of standard cells" by the Josephson junction; Gibney 2017: "Metrologists are poised to change how scientists measure the Universe"; Gibney 2018: "Metrologists ditch last physical standard units ... 'Le Grand K' ... will lose its special status"; Cartlidge 2018: "Metrologists move to redefine the second with a visible light standard that would boost accuracy by a factor of 100 ."

${ }^{332}$ Festinger 1957, quoting pp.42-43; Festinger 1955; Brehm 1956; Jarcho et al. 2011: "decision-related attitude change." The classical example is "sour grapes." Sometimes the "change" is entrenchment — see especially Festinger et al. 1956.

333 Thomas Carmine Beneventano, M.D., ca. 1980, in a chat with radiology residents at Montefiore Hospital, Bronx, NY. 
ASPECTS has already received diplomatically worded adverse assessments by researchers:

ASPECTS on NCCT is a long way down the pecking order.... Automated, validated volumetric measures of core and penumbra can now be generated rapidly. So, why bother with ASPECTS? These modern imaging techniques should confine ASPECTS to the museums. ${ }^{334}$

ASPECTS has limitations ... limited to the anterior circulation ... exact extent of each region or how much damaged tissue is required to render a region affected has never been defined ... regions cover different amounts of brain tissue ... same ASPECTS ... not necessarily have similar lesion volumes ... template's unequal weighing could compromise clinical decisions ... unjustified exclusion of patients from clinical trials or even treatment ... single threshold to identify patients with poor outcome based on the available data seems hardly justifiable. ${ }^{335}$

ASPECTS interpretation is less accurate in estimating the core volume than are other imaging methods, [citing $\left.{ }^{336}\right] \ldots .^{337}$

NCCT-ASPECTS may furthermore be limited by its tendency to cluster largely variable volumes of injury across its coarsely changing scale, ... [I] dentical NCCT-ASPECTS between 2 subjects can belie considerable differences in the actual volume of injury. ${ }^{338}$

Our study has some limitations. First, because of the use of DWI-ASPECTS for brain volume infarct evaluation rather than DWI volumetric measurements. ${ }^{339}$

$[T]$ his methodology is not completely standardized. The ASPECTS regions are imprecisely delineated, and it is not specified to what extent the region must be affected by EIC in order to warrant subtracting a point. ... ambiguities of the ASPECTS methodology ... ${ }^{340}$

We opted to adopt the RAPID automated software in order to optimize the standardization of the imaging inclusion criteria across the many different sites and increase the precision of the baseline core measurements. Notably, the other existent option at the time of the study design included local ASPECTS reading which would have theoretically added greater variability in the imaging assessment across sites. ${ }^{341}$

[T]he critical flaw is that ASPECTS is not a linear scale as the same score may have different meanings in terms of both volume and eloquence. ... This mismatch between ASPECTS and infarct volume results in many low ASPECTS patients actually having relatively small infarcts .... over half of the patients with ASPECTS $\leq 5$ will have an infarct volume $<100 \mathrm{~mL}$.... could lead to unjustified exclusion of patients from treatment. ${ }^{342}$

\footnotetext{
334 Bivard \& Parsons 2012, entitled: “ASPECTaSaurus (a dinosaur)?” (NCCT = noncontrast CT).

${ }^{335}$ Schröder \& Thomalla 2017, pp. 4-5 (some quoted segments reordered). Make that limited to the MCA circulation.

${ }^{336}$ McTaggart et al. 2015, e.g., p. 411: "DWI-ASPECTS scores have been shown to correspond to a wide range of DWI lesion volumes [citing Lin et al. 2011, de Margerie-Mellon et al. 2013]."

${ }^{337}$ Jovin et al. 2015 (REVASCAT), p. 2303. Jovin is also a coauthor of the incorporated citation.

338 Dehkharghani et al. 2016, p. 1402.

${ }^{339}$ Desilles et al. 2017, p. 968, also: "DWI-ASPECTS includes a wide variation of lesion volumes for each score value ...."

${ }^{340}$ Wilson et al. 2018, pp. 143-4. On region size heterogeneity, Wilson et al. are already quoted in \$6.3.

${ }^{341}$ Nogueira et al. 2018a (DAWN), Supplementary Appendix, p. 32, citing Farzin et al. 2016.

${ }^{342}$ Nogueira \& Ribó 2019, pp. 2615-6. Cf. Campbell 2019. Cf. §7's final case, finally done, but delayed by ASPECTS.
} 
Subjected to due diligence, ASPECTS proves hollow and neglectful on many levels:

1. Measuring infarct volume, for the purpose of gauging hemorrhage risk, was long held to be one of the important "Aspects of Stroke Imaging": "Initially the role of CT scanning was to exclude patients with haemorrhage and to audit the risk of haemorrhage following treatment. Most of our efforts have been focused on the risk of symptomatic haemorrhage following thrombolysis for stroke."343 But ASPECTS's variegated units break it as a measure (\$6). No matter how meticulously ASPECTS is enumerated, it is the wrong concept of a measure. That ASPECTS correlates with volume and with some clinical variables that correlate with volume, in a loose transitivity of correlation (\$2.5), is a lame excuse and a travesty of scientific measurement. Why would anyone invent or tolerate, ostensibly to measure volume, anything as distorting as ASPECTS?

2. The target volume of $1 / 3$ of the MCA territory was never a proper scientific measure because the MCA territory is not bounded by the imaging (\$4). Moreover, it was not reliably guessable because, as was known, its size varies a lot. Its average volume could have been stated for use, but was not.

3. The $1 / 3$ MCA rule for hemorrhage risk was only ever a mirage $(\$ 4)$. Its use as the target MCA fraction could have been characterized frankly as arbitrary, but instead was covered by citations in yet another example of the well known phenomenon of medical references not saying what they are cited for.

4. ASPECTS is MCA-only, not for any good reason in 2000, but from a misreading of ECASS (\$3.1).

5. As a concoction by six cooks, the ASPECTS methodology comes with a brazen conflict matrix (\$3.2). A few disambiguations have not made it a well-honed method, and some of these (like limiting "IC" to PLIC, or not limiting M1-6 to cortex) are overlooked by some writers and computer software.

6. ASPECTS was set up against the straw man (\$3.1) of "eyeball” volume guessing (\$4) but should have used a more comprehensive check list (11, below) and been compared to the known $A B C / 2$ (\$7).

7. Pretending that it matters less whether we're right (science) than whether we agree (politics), ASPECTS was then claimed to be more reliable (\$5) than its straw man just by interrater agreement, without considering accuracy. Reliability was unreliably measured by the simple $\boldsymbol{\kappa}$ statistic that, beyond strictly categorical (not "dichotomized") binary scales, ignores the loss function (\$6.2) inherent in degrees of error or disagreement - which in ASPECTS can be widely spread degrees.

8. ASPECTS (and volume) fell out of the calculus for intravenous tPA, but ASPECTS lingers as it fortuitously fits thrombectomy's MCA territory and tries to eclipse infarct volume for patient selection.

9. Much of ASPECTS's research thrives on groupthink, belief perseverance, and a kind of confirmation bias, although taking the latter not on the usual cognitive level ${ }^{344}$ but advanced to a procedural level that constructs a Woozle $(\$ 4$, Table 4.1$) .{ }^{345}$

\footnotetext{
343 Buchan 2001, title and p. 99.

344 e.g., Chapman \& Johnson 2002, p. 133: "decision makers examine evidence expected to confirm the hypothesis ...."

${ }^{345}$ Dutton 2006, pp. 109-110, relates the "woozle effect" to "confirmatory bias," "belief perseverance," and "groupthink." But compare: "The most perfect emblem of ignorance is contained in the 'Woozle' scene"-Meadowlark 1963, p. 78.
} 
10. Perhaps seeing the handwriting on the wall, inscribed by any or all of its failings listed above, some ASPECTS advocates tried by fiat to justify its overweighting by changing its mission (\$8). But, as if anticipating this mission creep, others had already pointed out that, with respect to the $1 / 3$ MCA rule, "the ASPECTS instrument ... is a refinement of that rule rather than a completely new development."346 It would be amazing if, by chance coincidence, ASPECTS's mere convenience sample of four small regions that CT happens to outline, and no others, turned out to deserve just this overweighting. Alas, research into the effects of infarcts in these and other regions shows otherwise.

11. It's a truism that "there is room to improve detection of $>33 \%$ MCA hypodensity,"347 as well as all infarcts, thrombosis, hemorrhage, and everything else. If "perhaps one of the biggest advantages of ASPECTS [is that] it forces the rater to look at every part of the MCA territory...," ${ }^{\prime 34}$ then why should we look at only the MCA territory and why should a check list be tallied as a volume? Pretending that all items in a verbal check list of namable regions have equal volumes is an error paralleling probability's largely discredited "principle of indifference."349 In the interest of being "systematic," 350 here is the helpfully comprehensive screening check list that ASPECTS is not:

a. Survey the whole head CT or MRI to look for intracranial, cranial, and extracranial pathology.

$b$. Survey especially meticulously for intra- and extraparenchymal hemorrhage.

c. Survey the carotid and vertebrobasilar systems for aneurysms and for arterial thrombi.

d. Inspect the insulae because their infarcts (by CT lucency) are often early and subtle.

$e$. Widen your cerebral cortical survey from the vertex to the inferior temporo-occipital lobes, with "a 'second look,' gyrus by gyrus, ... especially in clinically suspicious regions."351

$f$. Study the cerebral white matter down to the capsules.

g. Make sure the caudates, putamina, globi pallidi, and thalami are discernible and intact.

$h$. Study the midbrain, pons, peduncles, and medulla, and ask yourself about all the "artifacts?"

$i$. Look for violations of the regular cerebellar folial pattern, and verify the horizontal fissure.

$j$. Look again at the seemingly chronic white matter, capsuloganglionic, and pontine lacunes to see if any look (by CT) lighter and smoother than typically chronic or explained by volume averaging and might fit clinically as acute infarcts (often a difficult and daring judgment by CT).

12. The degradation of measurement introduced by ASPECTS in 2000 recalls the role of measurement in the 1999 loss of the Mars Climate Observer. "History does not repeat itself, but it rhymes,"352 so even without a precise analogy, Table 10.1 (next page) shows parallels between these fiascos.

\footnotetext{
${ }^{346}$ Schellinger et al. 2003, p. 576. And so it remains, "a surrogate of infarct volume"-Rangaraju et al. 2015, p. 1787.

347 Marks et al. 1999, p. 392.

${ }^{348}$ Coutts et al. 2003. Cf. Mak et al. 2003 also finding better infarct detection with ASPECTS, presumably guiding search.

${ }^{349}$ Keynes 1921, p. 41; p. 42: "that if there is no known reason for predicating of our subject one rather than another of several alternatives, then relatively to such knowledge the assertions of each of these alternatives have an equal probability." Keynes goes on to discuss the underlying fallacies, which often amount to unknown knowns, things we really know but forgot to consider - such as size differences.

350 quoting, e.g., Barber et al. 2000, pp. 1671, 1673; Pexman et al. 2001, pp. 1534, 1540; Puetz et al. 2009, pp. 354ff.

${ }^{351}$ Fox 2002, p. 1566.

352 O'Toole 2014: thought to date from the 1960s as "proverbial lore," the second part falsely attributed to Mark Twain.
} 
Table 10.1: Measurement errors compared.

\section{Mars Climate Observer}

Between Lockheed and NASA, different units were unlabeled and substituted:

Lockheed specified thruster force in pounds, NASA thought Lockheed meant newtons, ${ }^{353}$ which differ by a factor of 4.4482205 .

"Given expected errors in altitude targeting of about $10 \mathrm{~km}$, a spread of values over a $100-\mathrm{km}$ range should have people screaming down the halls." ${ }^{354}$

"[Y]ou have no idea where your spacecraft is."

"If JPL did real software configuration and control, the error would never have gotten by the door., ${ }^{356}$

"Although the navigators continued to express concerns about the spacecraft trajectory, ... 'They did not use the existing formal process for such concerns,' .... JPL has a special form to invoke a so-called incident surprise and error analysis procedure." ${ }^{157}$

"[A]dequate doubts had been raised ...."358

The mission was lost.

"That is so dumb." 359

"[I]t was embarrassing to lose a spacecraft to such a simple math error." ${ }^{160}$

\section{Alberta Stroke Program Early CT Score}

Within the ASPECTS scale, different units are tacitly mixed and summed:

large regions, little regions, which differ by a factor of 7-8 (\$6.1).

Given expected precision of infarct volume, the spread, for most volumes, of ASPECTS over a 6-point range (\$6.3) should have people screaming down the halls.

You have no idea what your infarct volume is.

If journals did insightful peer review and quality control, ASPECTS would never have gotten by the door.

Although this author continues to express concerns about the effects of ASPECTS, he has not used the existing formal process of peer-reviewed publication, but relies on conscientious doctors to use volume and avoid ASPECTS.

Adequate doubts have been raised (\$6).

Patients can be misclassified for \pm treatment and their mission can be lost.

"I think the scale is crap."

-a neurointerventionist, November 14, 2018

It is embarrassing to have to use ASPECTS or cite Pexman et al. in a radiology report.

\footnotetext{
353 Oberg 1999, p. 36.

354 “one navigation expert," quoted by Oberg 1999, p. 38.

355 evidently the same navigation expert, quoted by Oberg 1999, p. 38.

356 "One of [IEEE] Spectrum's chief sources," quoted by Oberg 1999, p. 39.

357 Oberg 1999, p. 38, with inner quote by NASA chief investigator Arthur Stephenson.

358 Oberg 1999, p. 39. "Concerns" and “doubts" can be understatements or euphemisms, as they are on the right.

359 John Logsdon, director of George Washington University's space policy institute, quoted by Hotz 1999.

360 John Pike, space policy director at the Federation of American Scientists, quoted by Hotz 1999.
} 
ASPECTS is an unnatural formality that parodies data theory by caricaturing content analysis, a research method for "converting verbal or other symbolic material [e.g., "pictures'] into numbers." Content analysis "break[s] up a 'communication' into bits that can be counted ... 'unitizing"' (the regions), and selects a "category-set ... classes ... into which the units of qualitative data may be placed"362 (any involvement vs.none). But "If units are not identified reliably, then the resulting content analysis will be unreliable," and "in selecting categories ... to avoid bias and idiosyncrasy one needs some kind of theoretical framework of reference."363 The pragmatic sense of "reliable" $(\$ 2.3, \S 5)$ describes something that can be relied on for critical use. For the true target of the analysis, which as shown in $\mathbf{\S 3 , 4 , 8}$ and attested up to the present ${ }^{364}$ is infarct volume, ASPECTS is critically defective in unitization (fuzzy definitions and diverse volumes) and categorization (2 biased categories per unit). Both reflect inappropriate theoretical frameworks to measure volume: ASPECTS is not a reliable content analysis, and content analysis is inappropriate anyway for an accessible continuous variable like volume.

ASPECTS has deviated from scrupulous methodological science to become an end in itself. Not satisfied to fulfill the prediction that "Fully automated planimetric assessment tools may one day allow accurate volume calculations in the hyperacute setting, ${ }^{, 365}$ the computer software e-ASPECTS ${ }^{\circledR}$ (https://brainomix.com/e-aspects) ${ }^{366}$ and RAPID ASPECTS ${ }^{\circledR}$ https://www.rapidai.com/rapid-aspects ${ }^{367}$ measure volume, but then report ASPECTS. After years of advocates calling ASPECTS "simple" or the like (above), now with e-ASPECTS on the horizon two articles about it say, "the required expertise that the manual application of ASPECTS carries can limit its use in clinical care," and "The ASPECTS score ... can be too difficult to use under pressure ..."- but e-ASPECTS could not process $25 \%$ of non-poor scans of anterior-circulation ischemia patients. ${ }^{368}$ Tepidly, "e-ASPECTS is non-inferior to three neuroradiologists in scoring ASPECTS ...."369 The elephant in the room is infarct volume.

Two studies compare RAPID ASPECTS ${ }^{\circledR}$ to manual ASPECTS. One claims, for small infarcts, better agreement to a consensus CT-ASPECTS by RAPID ${ }^{\circledR}$ CT-ASPECTS than by manual CT-ASPECTS, without comparing to volumes. The other claims, for large infarcts, better agreement to DWI (manual ASPECTS and planimetric volumes) by RAPID ${ }^{\circledR}$ CT-ASPECTS than by manual CT-ASPECTS. ${ }^{370}$ The inclusion of DWI planimetry as a gold standard in the latter reiterates that lesion volume really is primary. And the latter study replicates marked overlap of ASPECTS among volumes $(\S 6.3)$, a devastating ambiguity that can be precluded by using only volume.

\footnotetext{
${ }^{361}$ Hall \& Van de Castle 1966, p. 1; Sun 2017, p. 148 ('pictures'). Cf. more generally Krippendorff 2004 or 2019.

362 quoted respectively (italics added): Carney 1971, p. 52; Guetzkow 1950, p. 48. Cf. Suss \& Pinho 2020.

363 quoted respectively (italics added): Pavitt 2017, p. 1422; Carney 1971, p. 53.

364 El-Tawil et al. 2019, p. 3111: e.g., "core volume."

${ }^{365}$ Butcher et al. 2008, p. 79; cf. Campbell et al. 2019, Supp. appx. p. 54, favorable for RAPID (volume), with references.

366 "The original ASPECTS method was invented by Professor Alastair Buchan, a co-founder of Brainomix.... Brainomix already have commitment from hospitals in the UK, Germany and Finland ... and are aiming to secure approval in the USA by the end of 2016"-Brainomix 2015/2021. Sundaram et al. 2019 add Olea Sphere ${ }^{\circledR}$ for CTP (Brainomix 2018).

367 iSchemaView (n.d.) no longer says "not currently commercially available in the U.S."

368 respectively: Hampton-Till et al. 2015, p. 73 (quoted); Lo 2017 (quoted); Nagel et al. 2018, pp. 1408-9.

${ }^{369}$ Nagel et al. 2017, p. 616. Lesaffre 2008, p.150: "a non-inferiority trial [aims] only to show that an experimental treatment is not (much) worse than a standard treatment." Cf. Mauri \&D’Agostino 2017, Lin \& Saver 2019. Noninferiority is not transitive.

${ }^{370}$ respectively: Maegerlein et al. 2019; Albers et al. 2019 (Figure 2, p. 3279, shows scatter and overlap by ASPECTS).
} 
It is explained above how the use of infarct volume is consistent with the 2018-19 Guidelines. Who, then, knowing the volume, would want to fall back on ASPECTS at all, even automatically? ${ }^{371}$ Examples may include anyone with cognitive dissonance from having adopted the expensive software.

Even for automated volume - which could be valuable if it is at least as accurate and quick as $A B C / 2$ and $2 S h / 3$ - "a final human check for plausibility is needed, particularly in patient groups with pre-existing cerebral changes." "ASPECTS software is designed to be used in conjunction with an experienced reader to help validate the final score and to detect artifacts or technical issues ... a thorough review of the software output ... is mandatory prior to treatment decisions." ${ }^{372}$ This means that automatic volume, and of course its add-on, automatic ASPECTS, is still not completely automatic. Moreover, if there is any doubt about acute-infarct delineation, by eye or software, ASPECTS per se does nothing to fix this problem.

A therapeutic decision deserves better than an entrenchment of the misbegotten ASPECTS scale. Sophisticated neurointerventionists probably see through ASPECTS and prefer something closer to the true volume which, though they can't be up all night to do their own screening, they could request or may unconsciously or consciously be estimating for themselves to tweak any ASPECTS they are fed. One interventionist told me he would take up to half the average MCA territory-his own opinion, before he knew of these references. ${ }^{373}$ Some others, even working in an ASPECTS-bound institution, have told me they do look at volumes. Their decision should be based on relatively transparent, analytical biological parameters - infarct volume + clinical data — not a confused conflation score.

Insistence on ASPECTS would burden the thrombectomy service with a measure from \$2.2's purpose category 4 (pro forma). "A summary statistic is meant to be a tool for communication,"374 but ASPECTS is basically a 10-pixel camera (strike 1) with diversely sized pixels (strike 2), each saturated by one photon (any part, strike 3). ASPECTS can also be compared to a lens system that is out of focus and misaligned. Its image of an infarct is not a reliable thumbnail but a risky distortion. ASPECTS fails to measure up in the Great Ledger of stroke care.

The successes of acute stroke care do not excuse the complacency of premature satisficing ${ }^{375}$ (being satisfied by what seems to suffice) with ASPECTS. ASPECTS not only "adds nothing" (not an independent predictor, $\$ \mathbf{4}, \S 8$ ), it subtracts value by competing against the true underlying measure, infarct volume. ASPECTS boggles the mind, but there is a cure. The following summaries I-III with their recommendations follow directly from the facts assembled in the prior sections.

\footnotetext{
${ }^{371}$ For example, there is no justification to specify, as suggested by Rothwell \& Buchan 2012, the use of ASPECTS in a "mobile stroke unit" (a CT-equipped ambulance), probably requiring automatic assessment by e-ASPECTS or the like to "potentially increase neuroradiological competence during the prehospital phase" (Grunwald et al. 2016, p. 333), when the same software could read out the volume of "the "tissue window"" (Rothwell \& Buchan 2012, p. 383). Abandoning ASPECTS, Sheth et al. 2019 developed for CTASI an automated ischemic core volume determination.

372 quoted respectively: Guberina et al. 2018, pp. 889,900; Maegerlein et al. 2019, pp. 147-8 (emphasis added). For CTP, Vagal et al. 2019 discuss automation's pathophysiological and technical pitfalls.

373 cf.: Han et al. 2016, p. 6, suggests DWI $150 \mathrm{~cm}^{3}$ as a threshold for futility; Campbell et al. 2019, p. 53: "up to $150 \mathrm{~mL}$." ${ }^{374}$ Maclure \& Willett 1987, p. 168. Example: an omnibus index, §8.

${ }^{375}$ Simon 1956, pp.129,136; - 1997, pp.118-20. $A B C / 2$ and $2 S h / 3$ (\$7, esp. note ${ }^{240}$ ) reflect satisficing too, but now with much better optimization between effort and results, as an administrative practicality countenanced by Simon.
} 


\section{Research}

ASPECTS is not a research-grade "tool." "Metrology is Key to Reproducing Results,"376 but an unreliable measurement of an ill-conceived measure does not fulfill Kelvin's doctrine (\$1). ASPECTS is not fixed by passing it off as computer software, masking interrater variability by using only one rater, and masking volume-inaccuracy by scoring with black-box authority. ASPECTS guidelines can be converted to reasonably corresponding volumes by using the mean mean method (\$9). If such a conversion were thought to be difficult or inadequate, that would be because ASPECTS is too coarse and incommensurate with volume and should never have been used in the first place. Researchers should recognize what a few cited above have articulated about ASPECTS's inappropriateness. Their protocols should require volume measurements, whether automated, semiautomated, or manual by $A B C / 2$ or $2 S h / 3$, in order to be lucidly, not just loosely, quantitative. Funding agencies should insist on no less.

\section{Practice}

ASPECTS, whether manual or automatic (as software), has not been shown to be safe or effective beyond, or even equivalent to, a volume measure. Indeed, the evidence points the other way. Nor is it mandated from above (\$9). Its adoption in a clinical site's protocol is self-inflicted and reversible. If the front line are required to toe the line with ASPECTS, §6 gives an idea of what to expect. Patients have sued for not getting $\mathrm{tPA}^{377}$ and may be displeased if wrongly denied thrombectomy - now "the standard of care in patients with LVO related acute stroke."378 ASPECTS training should be switched to $A B C / 2$ and $2 S h / 3$ training using a check list like \#11 $a-j$. The requisite arithmetical skill is available on demand. Test Question: Would you stand on the ceremony of a low ASPECTS when it misrepresents a small infarct, to deny thrombectomy and leave the core to its natural history of expanding across the "metastable" penumbra?

\section{Research About Research}

Based on the literature and data assembled in this article, the only justified use of ASPECTS is as a cautionary tale of poorly vetted writings propagating a strange idea.

We need to enforce a diligent synthesis of evidence and sense, an effective peer review system, ${ }^{380}$ and an ethic of thorough, critical reading - to promote more insightful investigation and exposition. ASPECTS's dysfunctional causes and effects - its coarse, doubly biased scale $(\S 3, \S 6)$, its testimonials to mere correlations uncontested by better measures $(\$ 4)$ and to interrater agreement unmoored from accuracy (\$5), and its fogging of data and decisions (\$6) - favor doctors who do not use it and patients on whom it is not used. We owe them the better measure that we have known all along: simple, straightforward, reliable, validated, useful, helpful, easy, and quick infarct volume.

\footnotetext{
376 Sené et al. 2017, title.

377 Avitzur 2006; Weintraub 2006; Liang \& Zivin 2008; Bruce et al. 2011; Bhatt et al. 2013; Miller \& Zois, LLC 2018.

378 Turc et al. 2019, p. 535; “standard of care”: Goyal et al. 2016a, p. 1723; Goyal et al. 2019, p. 3; Nogueira \& Ribó 2019 (phrase not used, but supportive); Haslett et al. 2019, p. 2858; p. 2860, 7 suits resolved 2009-16, 4 with payments.

379 Ginsberg \& Pulsinelli 1994, p. 553 (quoted); cf. Hossmann 1994, Fisher 1997, Parsons et al. 2005.

380 D'Andrea \& O’Dwyer 2017, p. 1: “the biggest hazard ... indifferent acceptance of low-quality [manuscripts]."
} 


\section{References}

Journal abbreviations: $A J N R=$ American Journal of Neuroradiology; BMJ = British Medical Journal (official names: $1988 \rightarrow B M J, 2014 \rightarrow$ The BMJ);EMJ =European Medical Journal; JAMA=Journal of the American Medical Association; $J N N P=$ Journal of Neurology, Neurosurgery and Psychiatry; JSCD = Journal of Stroke and Cerebrovascular Diseases; NEJM = New England Journal of Medicine. This Adobe PDF could not hyperlink URLs containing parentheses.

Abbott AL, et al. (2017), "Optimizing the Definitions of Stroke, Transient Ischemic Attack, and Infarction for Research and Application in Clinical Practice," Frontiers in Neurology 8, Art. 537, https://doi.org/10.3389/fneur.2017.00537.

ACR et al. (2021), "ACR-ASNR-SIR-SNIS Practice Parameter for the Performance of Endovascular Embolectomy and Revascularization in Acute Stroke," American College of Radiology,

https://www.acr.org/-/media/ACR/Files/Practice-Parameters/Acute-Stroke.pdf.

Adams HP, et al. (1996), "Guidelines for Thrombolytic Therapy for Acute Stroke: A Supplement to the Guidelines for the Management of Patients with Acute Ischemic Stroke: A Statement for Healthcare Professionals from a Special Writing Group of the Stroke Council, American Heart Association," Circulation 94, 1167-1174, and Stroke 27, 1711-1718, https://doi.org/10.1161/01.cir.94.5.1167, https://www.ncbi.nlm.nih.gov/pubmed?linkname=pubmed pubmed\&from uid=8784157. Agresti A (1976), "The Effect of Category Choice on Some Ordinal Measures of Association," Journal of the American Statistical Association 71, 49-56, https://doi.org/10.1080/01621459.1976.10481475

Albers GW, et al. (2006), "Magnetic Resonance Imaging Profiles Predict Response to Early Reperfusion: The Diffusion and Perfusion Imaging Evaluation for Understanding Stroke Evolution (DEFUSE) Study," Annals of Neurology 60, 508-517, https://doi.org/10.1002/ana.20976.

Albers GW, et al. (2018), “Thrombectomy for Stroke at 6 to 16 Hours with Selection by Perfusion Imaging," NEJM 378, 708-718, https://doi.org/10.1056/NEJMoa1713973.

Albers GW (2019), via email to the author, Jun 18.

Albers GW, et al. (2019), "Automated Calculation of Alberta Stroke Program Early CT Score: Validation in Patients with Large Hemispheric Infarct,” Stroke 50, 3277-3279 + online supplement, https://doi.org/10.1161/STROKEAHA.119.026430. Alexander LD, et al. (2012), "Long-Term Prediction of Functional Outcome After Stroke Using the Alberta Stroke Program Early Computed Tomography Score in the Subacute Stage," JSCD 21, 737-744,

https://doi.org/10.1016/i.jstrokecerebrovasdis.2011.03.010.

Allen MJ, Yen WM (1979), Introduction to Measurement Theory, Monterey CA, Brooks/Cole.

Almekhlafi MA, et al. (2019), "Stroke Laterality Did Not Modify Outcomes in the HERMES Meta-Analysis of Individual Patient Data of 7 Trials," Stroke 50, 2118-2124, https://doi.org/10.1161/STROKEAHA.118.023102.

Amukotuwa S, et al. (2019), "Cerebral Blood Flow Predicts the Infarct Core: New Insights From Contemporaneous Diffusion and Perfusion Imaging," Stroke 50, 2783-2789, https://doi.org/10.1161/STROKEAHA.119.026640.

Anscombe FJ (1973), “Graphs in Statistical Analysis," The American Statistician 27, 17-21, https://doi.org/10.2307/2682899. Aoki J, et al. (2013), "DWI-ASPECTS as a Predictor of Dramatic Recovery After Intravenous Recombinant Tissue Plasminogen Activator Administration in Patients With Middle Cerebral Artery Occlusion," Stroke 44, 534-537, https://doi.org/10.1161/STROKEAHA.112.675470.

Apollo Medical Imaging Technology (2019), “AutoMIStar," https://www.apollomit.com/automistar.htm.

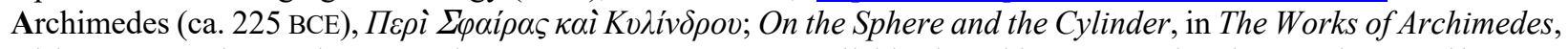
ed. by T. L. Heath, London: C. J. Clay \& Sons, 1897, pp. 1-90; available via archive.org; reprinted as supplemented in 1912, New York: Dover (n.d.); reprinted, Mineola NY: Dover, 2002.

Aristotle (ca. 350 BCE), "Prior Analytics," Aristotle, vol. I, trans. by H. Tredennick, Loeb Classical Library, Harvard University Press, 1938, 181-531.

Armitage P, Berry G (1987), Statistical Methods in Medical Research, $2^{\text {nd }}$ ed., Oxford: Blackwell.

Armitage P, Berry G (1994), Statistical Methods in Medical Research, $3^{\text {rd }}$ ed., Oxford: Blackwell.

Armitage P, et al. (2002), Statistical Methods in Medical Research, $4^{\text {th }}$ ed., Oxford: Blackwell.

Arsava EM, et al. (2008), "An Infarct Volume Threshold on Early DWI to Predict Unfavorable Clinical Outcome," International Stroke Conference Poster Presentations, P200, Stroke 39, 619, https://doi.org/10.1161/STROKEAHA.107.100001. Asdaghi N, et al. (2014), "DWI Reversal Is Associated with Small Infarct Volume in Patients with TIA and Minor Stroke," AJNR 35, 660-666, https://doi.org/10.3174/ainr.A3733.

Astrup J, et al. (1981), “Thresholds in Cerebral Ischemia-The Ischemic Penumbra," Stroke 12, 723-725, https://doi.org/10.1161/01.STR.12.6.723.

Athauda D, Tan GS (2014), "Evolving Infarction in the Anterior Circulation," NEJM 371, e20, https://doi.org/10.1056/NEJMicm1313055.

Augustine JR (1996), "Circuitry and Functional Aspects of the Insular Lobe in Primates Including Humans," Brain Research Reviews 22, 229-244, https://doi.org/10.1016/s0165-0173(96)00011-2.

Avitzur O (2006), "As Public Expectation for tPA Grows, So Too Do Lawsuits; How Neurologists Can Reduce Malpractice Risks," Neurology Today 6:9 (May 2), 31-32, https://doi.org/10.1097/00132985-200605020-00014. 
Page 80 ASPECTS, The Mismeasure of Stroke: A Metrological Investigation

Aviv R, et al. (2007), “Alberta Stroke Program Early CT Scoring of CT Perfusion in Early Stroke Visualization and Assessment," AJNR 28, 1975-1980, https://doi.org/10.3174/ajnr.A0689.

Baik SH, et al. (2019), "Mechanical Thrombectomy in Subtypes of Basilar Artery Occlusion: Relationship to Recanalization Rate and Clinical Outcome," Radiology 291, 730-737, https://doi.org/10.1148/radiol.2019181924.

Baizer JS, et al. (2014), "Comparative Organization of the Claustrum: What Does Structure Tell Us About Function?" Frontiers in Systems Neuroscience 8, article 117, https://doi.org/10.3389/fnsys.2014.00117.

Bakeman R, Gottman JM (1997), Observing Interaction: An Introduction to Sequential Analysis, $2^{\text {nd }}$ ed., Cambridge U. Press. Barak E, et al. (2008), "Does Hyperacute Diffusion and Perfusion Weighted Imaging Predict Outcome in Acute Ischemic Stroke?” International Stroke Conference Poster Presentations, P152, Stroke 39, 607, https://doi.org/10.1161/STROKEAHA.107.100001. Barber PA, et al. (2000), "Validity and Reliability of a Quantitative Computed Tomography Score in Predicting Outcome of Hyperacute Stroke Before Thrombolytic Therapy," The Lancet 355 (9216, May 13), 1670-1674+2170,

https://doi.org/10.1016/s0140-6736(00)02237-6.

Barber PA, et al. (2005), "Imaging of the Brain in Acute Ischaemic Stroke: Comparison of Computed Tomography and Magnetic Resonance Diffusion-Weighted Imaging," JNNP 76, 1528-1533, https://doi.org/10.1136/jnnp.2004.059261. Barlow HB (1961), "Possible Principles Underlying the Transformations of Sensory Messages," in W. A. Rosenblith, ed., Sensory Communication, Cambridge MA: MIT Press.

Bathla G, et al. (2017), "Calculating the Tumor Volumes in Vestibular Schwannomas: Are the ABC/2 and Volumetric Methods Comparable?” Otology \& Neurotology 38, 889-894, https://doi.org/10.1097/MAO.0000000000001423.

Beare R, et al. (2015), "Googling Stroke ASPECTS to Determine Disability: Exploratory Analysis from VISTA-Acute Collaboration," PLoS ONE 10, e0125687, https://doi.org/10.1371/journal.pone.0125687.

Ben-Naim A (2008), A Farewell to Entropy: Statistical Thermodynamics Based on Information, New Jersey: World Scientific. Bennett EM, et al. (1954), "Communications Through Limited Response Questioning," Public Opinion Quarterly 18, 303-308, https://doi.org/10.1086/266520.

Bentham J (1824), The Book of Fallacies. London: John and H. L. Hunt.

Berge E, et al. (2016), "Effects of Alteplase on Survival after Ischaemic Stroke (IST-3): 3 Year Follow-up of a Randomised, Controlled, Open-label Trial," Lancet Neurology 15, 1028-34, https://doi.org/10.1016/S1474-4422(16)30139-9.

Berkhemer OA, et al. (2015), “A Randomized Trial of Intraarterial Treatment for Acute Ischemic Stroke," NEJM 372, 11-20, https://doi.org/10.1056/NEJMoa1411587.

Beslow, LA, et al. (2010), "ABC/XYZ Estimates Intracerebral Hemorrhage Volume as a Percent of Total Brain Volume in Children," Stroke 41, 691-694, https://doi.org/10.1161/STROKEAHA.109.566430.

Beslow LA, et al. (2012), "Modified Pediatric ASPECTS Correlates with Infarct Volume in Childhood Arterial Ischemic Stroke," Frontiers in Neurology 3, Art. 122, https://doi.org/10.3389/fneur.2012.00122.

Bevan W (1953), "Modern Psychologists: Scientific Woozle Hunters? An Opinion in Outline," Nordisk Psykologi Monografiserie Nr. 4, Copenhagen: Einar Munksgaards Forlag.

Bhatia KP, Marsden CD (1994), "The Behavioural and Motor Consequences of Focal Lesions of the Basal Ganglia in Man," Brain 117, 859-876, https://doi.org/10.1093/brain/117.4.859.

Bhatt A, et al. 2013), "Medicolegal Considerations with Intravenous Tissue Plasminogen Activator in Stroke: A Systematic Review," Stroke Research and Treatment, Volume 2013, Article ID 562564, https://doi.org/10.1155/2013/562564.

Biomarkers Definitions Working Group (2001), "Biomarkers and Surrogate Endpoints: Preferred Definitions and Conceptual Framework," Clinical Pharmacology \& Therapeutics 69, 89-95; https://doi.org/10.1067/mcp.2001.113989.

Bivard A, Parsons M (2012), “ASPECTaSaurus (a dinosaur)?” International Journal of Stroke 7, 564, https://doi.org/10.1111/j.1747-4949.2012.00854.x.

Bivard A, Parsons M (2018), "Tissue Is More Important Than Time: Insights into Acute Ischemic Stroke from Modern Brain Imaging," Current Opinion in Neurology 31, 23-27, https://doi.org/10.1097/WCO.0000000000000520.

Bogousslavsky J, Regli F (1992) "Centrurn Ovale Infarcts: Subcortical Infarction in the Superficial Territory of the Middle Cerebral Artery," Neurology 42, 1992-1998, https://doi.org/10.1212/wnl.42.10.1992.

Boisseau W, et al. (2019), "Predictors of Parenchymal Hematoma After Mechanical Thrombectomy: A Multicenter Study," Stroke 50, 2364-2370, https://doi.org/10.1161/STROKEAHA.118.024512.

Bracard S, et al. (2016), "Mechanical Thrombectomy after Intravenous Alteplase Versus Alteplase Alone after Stroke (THRACE): a Randomised Controlled Trial,' Lancet Neurology 15, 1138-1147, https://doi.org/10.1016/S1474-4422(16)30177-6. Brainomix (2015/2021), "Brainomix Launches Revolutionary e-ASPECTS Software Designed to Improve Outcomes for Stroke Patients," https://brainomix.com/news/17, article dated April 13, 2015, Brainomix C2021, last accessed 2021.12.12. Brainomix (2018), "Brainomix and Olea Medical Join Forces to Revolutionise Stroke Imaging with the e-STROKE SUITE," https://www.prnewswire.com/news-releases/brainomix-and-olea-medical-join-forces-to-revolutionise-stroke-imaging-with-the-e-stroke-suite-682899991.html. Brainomix (2021), “Transforming Stroke Care Through Simple Imaging," https://brainomix.com/e-aspects, last accessed 2021.12.12. Brehm JW (1956), "Postdecision Changes in the Desirability of Alternatives," Journal of Abnormal and Social Psychology 52, 384-389, https://doi.org/10.1037/h0041006.

Brennan P, Silman A (1992), "Statistical Methods for Assessing Observer Variability in Clinical Measures," BMJ 304, 1491-1494, https://doi.org/10.1136/bmj.304.6840.1491. 
Brennan RL, Prediger DJ (1981), “Coefficient Kappa: Some Uses, Misuses, and Alternatives,” Educational and Psychological Measurement 41, 687-699, https://doi.org/10.1177/001316448104100307.

Broderick JP, et al. (1993), "Volume of Intracerebral Hemorrhage: A Powerful and Easy-to-Use Predictor of 30-Day Mortality," Stroke 24, 987-993, https://doi.org/10.1161/01.str.24.7.987.

Broderick JP, et al. (1994), [Letter] "Intracerebral Hemorrhage Volume Measurement," Stroke 25, 1081; https://pubmed.ncbi.nlm.nih.gov/8165684/.

Broderick JP, et al. (2013), "Endovascular Therapy after Intravenous t-PA versus t-PA Alone for Stroke," NEJM 368, 893-903 + Supplementary Appendix, https://doi.org/10.1056/NEJMoa1214300.

Brott T, et al. (1989), "Measurements of Acute Cerebral Infarction: A Clinical Examination Scale," Stroke 20, 864-870, https://doi.org/10.1161/01.str.20.7.864.

Bruce NT, et al. (2011), "Medico-Legal Aspects of Using Tissue Plasminogen Activator in Acute Ischemic Stroke," Current Treatment Options in Cardiovascular Medicine 13, 233-239, https://doi.org/10.1007/s11936-011-0122-0.

Brugnara G, et al. (2020), "Multimodal Predictive Modeling of Endovascular Treatment Outcome for Acute Ischemic Stroke Using Machine-Learning," Stroke 51, 3541-3551, https://doi.org/10.1161/STROKEAHA.120.030287.

Buchan A (2001), "Aspects of Stroke Imaging," Canadian Journal of the Neurological Sciences 28, 99-100, https://doi.org/10.1017/S0317167100052744.

Butcher KS, et al. (2005), "Increased Blood Volume Maintains Viability in Tissue With Isolated Focal Swelling on CT in Acute Stroke," American Stroke Association $30^{\text {th }}$ Annual Conference, New Orleans, February 2-4, Oral Presentations, Abstract 3; Stroke 36, 418, https://doi.org/10.1161/str.36.2.416.

Butcher KS, et al. (2007), "Differential Prognosis of Isolated Cortical Swelling and Hypoattenuation on CT in Acute Stroke," Stroke 38, 941-947, https://doi.org/10.1161/01.STR.0000258099.69995.b6.

Butcher KS, et al. (2008), "Rapid Assessment of Perfusion-Diffusion Mismatch," Stroke 39, 75-81, https://doi.org/10.1161/STROKEAHA.107.490524.

Byrne D, et al. (2019), "An Acute Stroke CT Imaging Algorithm Incorporating Automated Perfusion Analysis," Emergency Medicine 26,319-329, https://doi.org/10.1007/s10140-019-01675-2.

Campbell BCV, et al. (2015), "Endovascular Therapy for Ischemic Stroke with Perfusion-Imaging Selection,” NEJM372, 1009-1018, https://doi.org/10.1056/NEJMoa1414792.

Campbell BCV, et al. (2016), "Endovascular Thrombectomy for Stroke: Current Best Practice and Future Goals," Stroke and Vascular Neurology 1, e000004, http://doi.org/10.1136/svn-2015-000004.

Campbell BCV, et al. (2019), "Penumbral Imaging and Functional Outcome in Patients with Anterior Circulation Ischaemic Stroke Treated with Endovascular Thrombectomy Versus Medical Therapy: a Meta-analysis of Individual Patient-level Data," Lancet Neurology 18, 46-55 + Supp. Appx., https://doi.org/10.1016/S1474-4422(18)30314-4.

Campbell BCV (2019), "SELECTing Patients With Large Ischemic Core Who May Benefit From Endovascular Reperfusion," JAMA Neurology 76, 1140-1141, https://doi.org/10.1001/jamaneurol.2019.1789.

Carey SW (1988), Theories of the Earth and Universe: A History of Dogma in the Earth Sciences, Stanford U. Press. Carney TF (1971), "Content Analysis: A Review Essay," Historical Methods Newsletter 4, 52-61

https://doi.org/10.1080/00182494.1971.10593939.

Cartlidge E (2018), "Better Atomic Clocks Herald New Era of Timekeeping," Science 359, 968, https://doi.org/10.1126/science.359.6379.968.

Casscells W, et al. (1978), "Interpretation by Physicians of Clinical Laboratory Results," NEJM 299, 999-1001, https://doi.org/10.1056/NEJM197811022991808.

Castro Sotos AE, et al. (2009), “The Transitivity Misconception of Pearson's Correlation Coefficient," Statistics Education Research Journal 8, 33-55, https://www.stat.auckland.ac.nz/ iase/serj/SERJ8(2)_Sotos.pdf.

Chapman GB, Johnson EJ (2002), "Incorporating the Irrelevant: Anchors in Judgments of Belief and Value," in Gilovich T, et al., eds., Heuristics and Biases: The Psychology of Intuitive Judgement, Cambridge University Press, pp. 120-138.

Cheng B, et al. (2014), "Influence of Stroke Infarct Location on Functional Outcome Measured by the Modified Rankin Scale," Stroke 45, 1695-1702, https://doi.org/10.1161/STROKEAHA.114.005152.

Cherian S, et al. (2014), "Fatal Outcome of Tissue Plasminogen Activator Administration in a Stroke-like Syndrome," American Society of Neuroradiology, $52^{\text {nd }}$ Annual Meeting, Montreal, May 17-22.

Christensen H, et al. (2005), "Insular Lesions, ECG Abnormalities, and Outcome in Acute Stroke," JNNP 76, 269-271, https://doi.org/10.1136/jnnp.2004.037531.

Ciaffoni D (2017), "What is the 'Woozle effect', and Why Should We Be So Worried About It?"

https://medium.com/@davidciaffoni/what-is-the-woozle-effect-and-why-should-we-be-so-worried-about-it-1b6d25553b2e.

Cicchetti DV, Feinstein AR (1990), "High Agreement But Low Kappa: II. Resolving the Paradoxes," Journal of Clinical Epidemiology 43, 551-558, https://doi.org/10.1016/0895-4356(90)90159-m.

Ciccone A, et al. (2013), “Endovascular Treatment for Acute Ischemic Stroke,” NEJM 368, 904-913,

https://doi.org/10.1056/NEJMoa1213701.

Clark WM, et al. (1999), "Recombinant Tissue-Type Plasminogen Activator (Alteplase) for Ischemic Stroke 3 to 5 Hours After Symptom Onset. The ATLANTIS Study: A Randomized Controlled Trial,” JAMA 282, 2019-2026, https://doi.org/10.1001/jama.282.21.2019. 
Page 82 ASPECTS, The Mismeasure of Stroke: A Metrological Investigation

Cohen J (1960), "A Coefficient of Agreement for Nominal Scales," Educational and Psychological Measurement 20, 37-46, https://doi.org/10.1177/001316446002000104.

Cohen J (1968), "Weighted Kappa: Nominal Scale Agreement with Provision for Scaled Disagreement or Partial Credit," Psychological Bulletin 70, 213-219, https://doi.org/10.1037/h0026256.

Cohen JD, et al. (2010), "Morphometry of Human Insular Cortex and Insular Volume Reduction in Williams Syndrome," Journal of Psychiatric Research 44, 81-89, https://doi.org/10.1016/j.jpsychires.2009.07.001.

Corder GW, Foreman DI (2009), Nonparametric Statistics for Non-Statisticians: A Step-by-Step Approach, Hoboken NJ: Wiley.

Courant R, and John F (1989), Introduction to Calculus and Analysis, Volume I, New York: Springer-Verlag.

Coutts SB, et al. (2003), "ASPECTS Reading Requires Training and Experience," Stroke 34, e179 (letter), https://doi.org/10.1161/01.STR.0000092221.81498.91.

Coutts SB, et al. (2004), "Interobserver Variation of ASPECTS in Real Time," Stroke 35(5), e103-e105, https://doi.org/10.1161/01.STR.0000127082.19473.45.

Cox DR (1957), "Note on Grouping," Journal of the American Statistical Association 52, 543-547, https://doi.org/10.1080/01621459.1957.10501411.

Cruse A (2004), Meaning in Language: An Introduction to Semantics and Pragmatics, Oxford University Press.

Cuddon JA (1998), A Dictionary of Literary Terms and Literary Theory, Oxford UK: Blackwell.

Damasio H (1983), "A Computed Tomographic Guide to the Identification of Cerebral Vascular Territories," Archives of Neurology 40, 138-142, https://doi.org/10.1001/archneur.1983.04050030032005.

D'Andrea R, O’Dwyer JP (2017), “Can Editors Save Peer Review from Peer Reviewers?” PLoS ONE 12(10), e0186111. https://doi.org/10.1371/journal.pone.0186111.

Datta Banik S, et al. (2016), "Estimation of Stature from Hand and Foot Dimensions among University Girl-students in Bilaspur, Chhattisgarh, India," Anthropologie 54, 291-297, https://www.jstor.org/stable/26272564.

Davies TS (1840), Solutions of the Principal Questions of Dr. Hutton's Course of Mathematics, London: Longman, Orme. Davis SM, et al. (2008), "Effects of alteplase beyond $3 \mathrm{~h}$ after stroke in the Echoplanar Imaging Thrombolytic Evaluation Trial (EPITHET): a placebo-controlled randomised trial," Lancet Neurology 7, 299-309, https://doi.org/10.1016/S1474-4422(08)70044-9. Davis SM, Donnan GA (2014) "Time Is Penumbra: Imaging, Selection and Outcome: The Johann Jacob Wepfer Award 2014," Cerebrovascular Diseases 38, 59-72, https://doi.org/10.1159/000365503.

Decker S (2018), at Radiological Society of North America, Chicago, December 1-6, quoted by Klenske N (2019), “Teaching Residents Advanced Technology will 'Future-Proof' the Profession," RSNA News 29:1 (January), 7, https://rsna2018.rsna.org/dailybulletin/index.cfm?pg=18wed11.

De Haan R, et al. (1993), "A Comparison of Five Stroke Scales With Measures of Disability, Handicap, and Quality of Life," Stroke 24, 1178-1181, https://doi.org/10.1161/01.str.24.8.1178.

Dehkharghani S, et al. (2016), "Performance of CT ASPECTS and Collateral Score in Risk Stratification: Can Target Perfusion Profiles Be Predicted without Perfusion Imaging?" AJNR 37, 1399-1404, http://doi.org/10.3174/ajnr.A4727. Demaerschalk BM, et al. (2006), "ASPECT Scoring to Estimate >1/3 Middle Cerebral Artery Territory Infarction," Canadian Journal of the Neurological Sciences 33, 200-204, https://doi.org/10.1017/s0317167100004972.

de Margerie-Mellon C, et al. (2013), "Can DWI-ASPECTS Substitute for Lesion Volume in Acute Stroke?" Stroke 44, 3565-3567, https://doi.org/10.1161/STROKEAHA.113.003047.

Demchuk AM, et al. (2005), "Importance of Early Ischemic Computed Tomography Changes Using ASPECTS in NINDS rtPA Stroke Study," Stroke 36, 2110-2115, https://doi.org/10.1161/01.STR.0000181116.15426.58.

Demchuk AM, Coutts SB (2005), "Alberta Stroke Program Early CT Score in Acute Stroke Triage," Neuroimaging Clinics of North America 15:2(May, Stroke I), 409-419, https://doi.org/10.1016/j.nic.2005.06.008.

Demeestere J, et al. (2017), "Evaluation of Hyperacute Infarct Volume Using ASPECTS and Brain CT Perfusion Core Volume," Neurology 88, 2248-2253 + Supplementary Data, https://doi.org/10.1212/WNL.0000000000004028.

Demeestere J, et al. (2018), "Alberta Stroke Program Early CT Score Versus Computed Tomographic Perfusion to Predict Functional Outcome After Successful Reperfusion in Acute Ischemic Stroke," Stroke 49, 2361-2367, https://doi.org/10.1161/STROKEAHA.118.021961.

Demeestere J, et al. (2019), Reply to Letter by Harston et al. 2019 (re: Demeestere et al. 2018), Stroke 50, e21, https://pubmed.ncbi.nlm.nih.gov/30580744/.

Deming WE (1944), “On Errors in Surveys," American Sociological Review 9, 359-369, https://doi.org/10.2307/2085979.

Desilles J-P, et al. (2017), "Successful Reperfusion With Mechanical Thrombectomy Is Associated With Reduced Disability and Mortality in Patients With Pretreatment Diffusion-Weighted Imaging-Alberta Stroke Program Early Computed Tomography Score $\leq 6$," Stroke 48, 963-969, https://doi.org/10.1161/STROKEAHA.116.015202.

Dippel DWJ, et al. (1997), "The Validity and Reliability of Early Infarct Signs on CT in Acute Ischaemic Stroke." Cerebrovascular Diseases 7, Suppl 4, 15 (abstr); cited by Barber et al. 2000, p. 1670, 1674; article published as Dippel et al. 2000. Dippel DWJ, et al. (2000), "The Validity and Reliability of Signs of Early Infarction on CT in Acute Ischaemic Stroke," Neuroradiology 42, 629-633, https://doi.org/10.1007/s002340000369.

Donkin WF (1851), "On Certain Questions Relating to the Theory of Probabilities," The London, Edinburgh and Dublin Philosophical Magazine and Journal of Science [Fourth Series] 1, 353-368, available via books.google.com. 
Dsouza L, et al. (2019), “ABC/2 Estimation in Intracerebral Hemorrhage: a Comparison Study Between Emergency Radiologists and Emergency Physicians," American Journal of Emergency Medicine 37, 1818-1822, https://doi.org/10.1016/i.ajem.2018.12.036. Dutton DG (2006), Rethinking Domestic Violence, Vancouver: UBC Press.

Dzialowski I, et al. (2006), "Extent of Early Ischemic Changes on Computed Tomography (CT) Before Thrombolysis: Prognostic Value of the Alberta Stroke Program Early CT Score in ECASS II," Stroke 37, 973-978, https://doi.org/10.1161/01.STR.0000206215.62441.56.

El-Tawil S, et al. (2019), "Observer Agreement on Computed Tomography Perfusion Imaging in Acute Ischemic Stroke," Stroke 50, 3108-3114 + Supplementary material, https://doi.org/10.1161/STROKEAHA.119.026238.

Ernst M. et al. (2017), "Association of Computed Tomography Ischemic Lesion Location With Functional Outcome in Acute Large Vessel Occlusion Ischemic Stroke," Stroke 48, 2426-2433, https://doi.org/10.1161/STROKEAHA.117.017513. Ernst M, et al. (2018), "Impact of Ischemic Lesion Location on the mRS Score in Patients with Ischemic Stroke: A Voxel-Based Approach," AJNR 39, 1989-1994+E1-6, https://doi.org/10.3174/ajnr.A5821.

Ertekin T, et al. (2013), "Comparison of Two Methods for the Estimation of Subcortical Volume Using Magnetic Resonance Imaging: A Methodological Study," Surgical and Radiologic Anatomy 35, 301-309,

https://doi.org/10.1007/s00276-012-1036-6.

Evans JD. Straightforward Statistics for the Behavioral Sciences, Pacific Grove CA: Brooks/Cole, 1996.

Everitt BS (2006), The Cambridge Dictionary of Statistics, $3^{\text {rd }}$ ed., Cambridge University Press.

Fabrigar LR, et al. (1999), "Evaluating the Use of Exploratory Factor Analysis in Psychological Research," Psychological Methods 4, 272-299, https://doi.org/10.1037/1082-989X.4.3.272.

Fahed R, et al. (2018a), "DWI-ASPECTS (Diffusion-Weighted Imaging-Alberta Stroke Program Early Computed Tomography Scores) and DWI-FLAIR (Diffusion-Weighted Imaging-Fluid Attenuated Inversion Recovery) Mismatch in Thrombectomy Candidates: An Intrarater and Interrater Agreement Study," Stroke 49, 223-227 + Supplemental Material, https://doi.org/10.1161/STROKEAHA.117.019508.

Fahed R, et al. (2018b), “A Randomized Pragmatic Care Trial on Endovascular Acute Stroke Interventions (EASI): Criticisms, Responses, and Ethics of Integrating Research and Clinical Care," Trials 19, art. 508,

https://doi.org/10.1186/s13063-018-2870-6.

Fahmi F, et al. (2017), "Image Based Automated ASPECT Score for Acute Ischemic Stroke Patients," 5th International Conference on Instrumentation, Communications, Information Technology, and Biomedical Engineering (ICICI-BME) Bandung, 6-7 November 2017, https://doi.org/10.1109/ICICI-BME.2017.8537717.

Faigle R, et al. (2014), "Predictors of Critical Care Needs after IV Thrombolysis for Acute Ischemic Stroke," PLoS ONE 9(2), e88652, https://doi.org/10.1371/journal.pone.0088652.

Faigle R, et al. (2015), "Infarct Volume Predicts Critical Care Needs in Stroke Patients Treated with Intravenous Thrombolysis," Neuroradiology 57, 171-178, https://doi.org/10.1007/s00234-014-1453-9.

Fano RM (1949), The Transmission of Information, Research Laboratory of Electronics Technical Report No. 65, Cambridge MA: Massachusetts Institute of Technology.

Farzin B, et al. (2016), "Early CT Changes in Patients Admitted for Thrombectomy: Intrarater and Interrater Agreement," Neurology 87, 249-256 + Supplementary Material, https://doi.org/10.1212/WNL.0000000000002860.

Fedorov V, et al. (2009), "Consequences of Dichotomization," Pharmaceutical Statistics 8, 50-61, https://doi.org/10.1002/pst.331.

Feinstein AR, Cicchetti DV (1990), "High Agreement but Low Kappa: I. the Problems of Two Paradoxes," Journal of Clinical Epidemiology 43, 543-549, https://doi.org/10.1016/0895-4356(90)90158-1.

Feinstein AR (2002), Principles of Medical Statistics, Boca Raton FL: Chapman \& Hall / CRC.

Festinger L (1955), "The Relation Between Cognition and Action," paper read at Symposium on Cognition, Boulder, CO, May, cited by Brehm 1956, pp. 384, 389.

Festinger L, et al. (1956), When Prophecy Fails, Minneapolis: University of Minnesota Press; reprinted, New York: Harper Torchbooks, 1964.

Festinger L (1957), A Theory of Cognitive Dissonance, Stanford University Press.

Feynman RP, et al. (1963), The Feynman Lectures on Physics, Volume 1, Reading MA: Addison-Wesley.

Fiebach JB, et al. (2002), "CT and Diffusion-Weighted MR Imaging in Randomized Order: Diffusion-Weighted Imaging Results in Higher Accuracy and Lower Interrater Variability in the Diagnosis of Hyperacute Ischemic Stroke," Stroke 33, 2206-2210, https://doi.org/10.1161/01.str.0000026864.20339.cb.

Fiebach JB, et al. (2015), "Reliability of Two Diameters Method in Determining Acute Infarct Size. Validation as New Imaging Biomarker," PLoS ONE 10(10), e0140065, https://doi.org/10.1371/journal.pone.0140065.

Fink JN, et al. (2005), "Insular Cortex Infarction in Acute Middle Cerebral Artery Territory Stroke," Archives of Neurology 62, 1081-1085, https://doi.org/10.1001/archneur.62.7.1081.

Finlayson O, et al. (2013), "Interobserver Agreement of ASPECT Score Distribution for Noncontrast CT, CT Angiography, and CT Perfusion in Acute Stroke," Stroke 44, 234-236, https://doi.org/10.1161/STROKEAHA.112.665208.

Fiorelli M, et al. (1999), "Hemorrhagic Transformation Within 36 Hours of a Cerebral Infarct: Relationships with Early Clinical Deterioration and 3-month Outcome in the European Cooperative Acute Stroke Study I (ECASS I) Cohort," Stroke 30, 2280-2284, https://doi.org/10.1161/01.str.30.11.2280. 
Page 84 ASPECTS, The Mismeasure of Stroke: A Metrological Investigation

Fisher M(1997), “Characterizing the Target of Acute Stroke Therapy,” Stroke 28, 866-872, https://doi.org/10.1161/01.str.28.4.866. Fisher M, Goyal M (2018), "Variance of Imaging Protocols for Patients With Suspected Acute Ischemic Stroke Because of Large-Vessel Occlusion," Stroke 49, 1805-8, https://doi.org/10.1161/STROKEAHA.118.022191.

Flannery JJ (1970), "The Relative Effectiveness of Some Common Graduated Point Symbols in the Presentation of Quantitative Data," for presentation during a Symposium on the Influence of the Map User on Map Design to be held at Queens University, Ontario, Canada, from September 8 to September 10, 1970, https://doi.org/10.3138/J647-1776-745H-3667; an original typescript is at https://files.eric.ed.gov/fulltext/ED045469.pdf.

Fleiss JL (1971), "Measuring Nominal Scale Agreement Among Many Raters," Psychological Bulletin 76, 378-382, https://doi.org/10.1037/h0031619.

Fleiss JL (1981), Statistical Methods for Rates and Proportions, $2^{\text {nd }}$ ed., New York: Wiley.

Fox AJ (2002), Editorial Comment, Stroke 33, 1565-1567, appended to https://doi.org/10.1161/01.STR.0000018011.66817.41. Franke CL, et al. (1988), "The Best Fit Method. A Simple Way for Measuring the Volume of an Intracerebral Haematoma," Neuroradiology 30, 73-75, https://doi.org/10.1007/BF00341948.

French KF, et al. (2014), "Reproducibility of ABC/2 Method to Determine Infarct Volume and Mismatch Percentage with CT Perfusion," Journal of Neuroimaging 24, 232-237, https://doi.org/10.1111/jon.12001.

Fukuda K, et al. (2017), "Utility of Aspects Region Location in Predicting Stroke Thrombectomy Outcomes," Journal of Neurointerventional Surgery 9(Suppl1) A3-A4, https://doi.org/10.1136/neurintsurg-2017-SNIS.5.

Furlan A, et al. (1999), "Intra-arterial Prourokinase for Acute Ischemic Stroke. The PROACT II Study: A Randomized Controlled Trial," JAMA 282, 2003-2011, https://doi.org/10.1001/jama.282.21.2003.

Galton F (1877), "Typical Laws of Heredity," Nature 15, 492-495, 512-514, 532-533; reprinted in Notices of the Proceedings at the Meetings of the Members of the Royal Institution of Great Britain with Abstracts of the Discourses Delivered at the Evening Meetings [Journal of the Royal Institution of Great Britain] 8 (1875-1878), London: William Clowes and Sons, 1879, pp. 282-301. Galton F (1888), "Co-relations and their Measurement, chiefly from Anthropometric Data," Proceedings of the Royal Society of London 45, 135-145.

Garcia JH, et al. (1996), “Ischemic Stroke and Incomplete Infarction," Stroke 27, 761-765 (unpaginated html in archive), https://doi.org/10.1161/01.str.27.4.761.

Gebel JM, et al. (1998), "Comparison of the ABC/2 Estimation Technique to Computer-Assisted Volumetric Analysis of Intraparenchymal and Subdural Hematomas Complicating the GUSTO-1 Trial," Stroke 29, 1799-1801,

https://doi.org/10.1161/01.str.29.9.1799.

Gelles RJ (1980), "Violence in the Family: A Review of Research in the Seventies," Journal of Marriage and the Family 42:4, 873-885, https://doi.org/10.2307/351830.

Gelman A, Stern H (2006), “The Difference Between 'Significant' and 'Not Significant' is not Itself Statistically Significant," The American Statistician 60, 328-331, https://doi.org/10.1198/000313006X152649.

Gibbs RW (1993), "Process and Products in Making Sense of Tropes," in Ortony A, ed, Metaphor and Thought, $2^{\text {nd }}$ ed., Cambridge University Press, Chapter 12, pp. 252-276.

Gibney E (2017), "New Definitions of Scientific Units Are on the Horizon," Nature 550,312-313, https://doi.org/10.1038/550312a.

Gibney E (2018), "Metrologists Ditch Last Physical Standard Units," Nature 563,451-452, https://doi.org/10.1038/d41586-018-07424-8.

Giles E, Vallandigham PH (1991), "Height Estimation from Foot and Shoeprint Length," Journal of Forensic Sciences 36, 1134-1151, https://www.ncbi.nlm.nih.gov/pubmed?linkname=pubmed pubmed\&from uid=1919473.

Ginsberg MD, Pulsinelli WA (1994), "The Ischemic Penumbra, Injury Thresholds, and the Therapeutic Window for Acute Stroke," Annals of Neurology 36, 553-554, https://doi.org/10.1002/ana.410360402.

Ginsburg HP, Opper S (1988), Piaget's Theory of Intellectual Development, $3^{\text {rd }}$ ed., Englewood Cliffs NJ: Prentice Hall. Goghari VM, et al. (2010), "Anterior Internal Capsule Volumes Increase in Patients with Schizophrenia Switched from Typical Antipsychotics to Olanzapine," J Psychopharmacology 25, 621-629, https://doi.org/10.1177/0269881110363314. Goldstein JH, et al. (2016), "Intra-Arterial Therapy for Acute Stroke and the Effect of Technological Advances on Recanalization: Findings in a Community Hospital," North Carolina Med J77,79-86, https://doi.org/10.18043/ncm.77.2.79. Goldstein LB, et al. (1989), "Interrater Reliability of the NIH Stroke Scale," Archives of Neurology 46, 660-662, https://doi.org/10.1001/archneur.1989.00520420080026.

Gomez CR (1993), “Time Is Brain!” JSCD 3, 1-2, https://doi.org/10.1016/S1052-3057(10)80125-9.

Gomez CR (2018), “Time Is Brain: The Stroke Theory of Relativity,” JSCD 27, 2214-2227,

https://doi.org/10.1016/j.jstrokecerebrovasdis.2018.04.001.

Gómez-Mariño R, et al. (2001), "Determinação Volumetrica do Infarto Cerebral na Fase Aguda Usando Tomografia Computadorizada de Crânio sem Contraste: Estudo comparando três métodos," Arquivos de Neuro-Psiquiatria 59, 380-383, https://doi.org/10.1590/S0004-282X2001000300013.

González RG, et al. (2012), "Improved Outcome Prediction Using CT Angiography in Addition to Standard Ischemic Stroke Assessment: Results from the STOPStroke Study," PLoS ONE 7(1), e30352, https://doi.org/10.1371/journal.pone.0030352. González RG, et al. (2013), "The Massachusetts General Hospital Acute Stroke Imaging Algorithm: An Experience and Evidence Based Approach,” Journal of Neurointerventional Surgery 5, i7-i12, https://doi.org/10.1136/neurintsurg-2013-010715. 
Good IJ (1950), Probability and the Weighing of Evidence, London: Charles Griffin.

Gould SG (1981), The Mismeasure of Man, New York: W. W. Norton; revised and expanded edition, 1996.

Goyal M, et al. (2015), "Randomized Assessment of Rapid Endovascular Treatment of Ischemic Stroke," NEJM 372, 1019-1030, https://doi.org/10.1056/NEJMoa1414905.

Goyal M, et al. (2016a), "Endovascular Thrombectomy After Large-Vessel Ischaemic Stroke: a Meta-analysis of Individual Patient Data from Five Randomised Trials," The Lancet 387, 1723-1731 + Supplementary Appendix,

https://doi.org/10.1016/S0140-6736(16)00163-X.

Goyal M, et al. (2016b), "Recent Endovascular Trials: Implications for Radiology Departments, Radiology Residency, and Neuroradiology Fellowship Training at Comprehensive Stroke Centers," Radiology 278, 642-645, https://doi.org/10.1148/radiol.2015151965.

Goyal M, et al. (2018), “How Do You Manage Cases with Low ASPECTS?” Endovascular Today 17:2 (Feb.), 74-78.

Goyal M, et al. (2019), "Which Patients with Acute Stroke Due to Proximal Occlusion Should Not Be Treated With Endovascular Thrombectomy?” Neuroradiology 61,3-8, https://doi.org/10.1007/s00234-018-2117-y.

Grandy WT (2008), Entropy and the Time Evolution of Macroscopic Systems, Oxford University Press.

Griffin AL (2017), "Cartography, Visual Perception and Cognitive Psychology," in Kent AJ, Vujakovic P, eds., The Routledge Handbook of Mapping and Cartography, Abingdon: Routledge, Chapter 3, pp. 44-54; available at https:// www.routledgehandbooks.com/doi/10.4324/9781315736822.ch3.

Grivas TB, et al. (2008), "Correlation of Foot Length with Height and Weight in School Age Children," Journal of Forensic and Legal Medicine 15, 89-95, https://doi.org/10.1016/i.jflm.2007.05.007.

Grotta JC, et al. (1999), "Agreement and Variability in the Interpretation of Early CT Changes in Stroke Patients Qualifying for Intravenous rtPA Therapy,” Stroke 30, 1528-1533, https://doi.org/10.1161/01.str.30.8.1528.

Grunwald IQ, et al. (2016), "First Automated Stroke Imaging Evaluation via Electronic Alberta Stroke Program Early CT Score in a Mobile Stroke Unit," Cerebrovascular Diseases 42, 332-338, https://doi.org/DOI:10.1159/000446861. Guberina N, et al. (2018), "Detection of Early Infarction Signs with Machine Learning-based Diagnosis by Means of the Alberta Stroke Program Early CT Score (ASPECTS) in the Clinical Routine," Neuroradiology 60, 889-901, https://doi.org/10.1007/s00234-018-2066-5.

Guetzkow H (1950), "Unitizing and Categorizing Problems in Coding Qualitative Data," Journal of Clinical Psychology 6, 47-58, https://doi.org/10.1002/1097-4679(195001)6:1<47::AID-JCLP2270060111>3.0.CO;2-I.

Guo B, et al. (2017), Alberta STE Report: Endovascular Therapy for Acute Ischemic Stroke, Edmonton AB: Institute of Health Economics (corresponding author identified on p. i, authors listed on p. 267),

https://www.ncbi.nlm.nih.gov/pubmed?linkname=pubmed pubmed\&from uid=29708695.

Gupta AC, et al. (2012), "Interobserver Reliability of Baseline Noncontrast CT Alberta Stroke Program Early CT Score for Intra-Arterial Stroke Treatment Selection," AJNR 33, 1046-1049, https://doi.org/10.3174/ajnr.A2942.

Guttman L (1946), “The Test-Retest Reliability of Qualitative Data," Psychometrika 11, 81-95,

https://doi.org/10.1007/bf02288925.

Hacke W, et al. (1995), "Intravenous Thrombolysis with Recombinant Tissue Plasminogen Activator for Acute Hemispheric Stroke: The European Cooperative Acute Stroke Study (ECASS)," JAMA 274, 1017-1025,

https://www.ncbi.nlm.nih.gov/pubmed?linkname=pubmed pubmed\&from uid=7563451.

Hacke W, et al. (1998), "Randomized Double-blind Placebo-controlled Trial of Thrombolytic Therapy with Intravenous Alteplase in Acute Ischaemic Stroke (ECASS II)," The Lancet 352(9136),1245-1251,

https://doi.org/10.1016/S0140-6736(98)08020-9.

Hacke W, et al. (2008), “Thrombolysis with Alteplase 3 to 4.5 Hours after Acute Ischemic Stroke,” NEJM 359, 1317-1329

+ Supplementary Appendix, https://doi.org/10.1056/NEJMoa0804656.

Hairer E, Wanner G (1996), Analysis by Its History, New York: Springer.

Hakonen P, Lounasmaa OV (1994), "Negative Absolute Temperatures: 'Hot' Spins in Spontaneous Magnetic Order," Science 2265, 1821-1825, https://doi.org/10.1126/science.265.5180.1821.

Haley MD, et al. (2018), "Retrospective Methods Analysis of Semiautomated Intracerebral Hemorrhage Volume Quantification From a Selection of the STICH II Cohort," Stroke 49, 325-332 + Supp., https://doi.org/10.1161/STROKEAHA.117.016677. Hall CS, Van de Castle RL (1966), The Content Analysis of Dreams, New York: Appleton-Century-Crofts.

Halliwell JO (1842, 1844), The Nursery Rhymes of England, London: Richards, and $3^{\text {rd }}$ ed., London: John Russell Smith; both available via books.google.com.

Hampton-Till J, et al. (2015), "Automated Quantification of Stroke Damage on Brain Computed Tomography Scans: e-ASPECTS," EMJ Neurology 3, 69-74, https://www.emjreviews.com/neurology/article/automated-quantification-ofstroke-damage-on-brain-computed-tomography-scans-e-aspects/.

Han M, et al. (2016), "Cerebral Infarct Volume Measurements to Improve Patient Selection for Endovascular Treatment," Medicine 95(35), Art. e4702, https://doi.org/10.1097/MD.0000000000004702.

Hanson NR (1960), “"The Mathematical Power of Epicyclical Astronomy," Isis 51, 150-158, https://doi.org/10.1086/348869. Haranhalli N, et al. (2020), "Topographic Correlation of Infarct Area on CT Perfusion with Functional Outcome in Acute Ischemic Stroke," Journal of Neurosurgery 132, 33-41, https://doi.org/10.3171/2018.8.JNS181095. 
Page 86 ASPECTS, The Mismeasure of Stroke: A Metrological Investigation

Harman WW (1963), Principles of the Statistical Theory of Communication, New York: McGraw-Hill.

Harston G, et al. (2019), Letter (re: Demeestere et al. 2018), Stroke 50, e20, https://doi.org/10.1161/STROKEAHA.118.023749.

Hartwig F, Dearing BE (1979), Exploratory Data Analysis, Beverly Hills CA: Sage Publications.

Haslett JJ, et al. (2019), "Systematic Review of Malpractice Litigation in the Diagnosis and Treatment of Acute Stroke," Stroke 50, 2858-2864, https://doi.org/10.1161/STROKEAHA.119.025352.

Haussen DC, et al. (2016), "Automated CT Perfusion Ischemic Core Volume and Noncontrast CT ASPECTS (Alberta Stroke Program Early CT Score): Correlation and Clinical Outcome Prediction in Large Vessel Stroke," Stroke 47, 2318-2322, https://doi.org/10.1161/STROKEAHA.116.014117.

Hayes AF, Krippendorff K (2007), "Answering the Call for a Standard Reliability Measure for Coding Data," Communication Methods and Measures 1, 77-89, https://doi.org/10.1080/19312450709336664.

Henning C, Kutlukaya M (2007), "Some Thoughts About the Design of Loss Functions," REVSTAT - Statistical Journal 5, 19-39, https://www.ine.pt/revstat/pdf/rs070102.pdf.

Herweh C, et al. (2016), "Performance of e-ASPECTS software in comparison to that of stroke physicians on assessing CT scans of acute ischemic stroke patients," International Journal of Stroke 11, 438-435,

https://doi.org/10.1177/1747493016632244.

Hilbert D, Cohn-Vossen S (1932), Anschauliche Geometrie, Berlin: Julius Springer; trans. by Nemenyi P, Geometry and the Imagination, New York: Chelsea, 1952, reprinted, 1990.

Hill MD, et al. (2003a), "Selection of Acute Ischemic Stroke Patients for Intra-Arterial Thrombolysis With Pro-Urokinase by Using ASPECTS," Stroke 34, 1925-1931, https://doi.org/10.1161/01.STR.0000082483.37127.D0.

Hill MD, et al. (2003b), Oral Abstracts, P138, "Inter- and Intra-Observer Reliability of ASPECTS," Stroke 34, 281-282, https://doi.org/10.1161/str.34.1.238.

Hill MD, Buchan AM (2005), "Thrombolysis for Acute Ischemic Stroke: Results of The Canadian Alteplase for Stroke Effectiveness Study," Canadian Medical Association Journal 172, 1307-1312, https://doi.org/10.1503/cmaj.1041561. Hill MD, et al. (2006), "Using the Baseline CT Scan to Select Acute Stroke Patients for IV-IA Therapy," AJNR 27, 1612-1616, https://www.ncbi.nlm.nih.gov/pubmed?linkname=pubmed pubmed\&from uid=16971597.

Hill MD, et al. (2014), "Alberta Stroke Program Early Computed Tomography Score to Select Patients for Endovascular Treatment," Stroke 45, 444-449 + Supplementary Material, https://doi.org/10.1161/STROKEAHA.113.003580.

Hoehn L, Niven I (1985), “Averages on the Move," Mathematics Magazine 58, 151-156,

https://doi.org/10.1080/0025570X.1985.11977173.

Honarmand AR (2013), "High Baseline CT ASPECTS Correlates With Perfusion-Based Patient Selection for Intra-arterial Recanalization in Acute Ischemic Stroke, but May Be Poor Discriminator of Functional Outcomes," Circulation 128, Supp. 22, Abstract 17946, https://www.ahajournals.org/doi/10.1161/circ.128.suppl 22.A17946.

Hopper PJ, Traugott EC (1993), Grammaticalization, Cambridge University Press.

Hossmann K-A (1994), "Viability Thresholds and the Penumbra of Focal Ischemia," Annals of Neurology 36, 557-565, https://doi.org/10.1002/ana.410360404.

Hotz RL (1999), "Mars Probe Lost Due to Simple Math Error," Los Angeles Times, October 1, https://www.latimes.com/archives/la-xpm-1999-oct-01-mn-17288-story.html.

Houghton BD (1979), "Review of Research on Women Abuse," American Society of Criminology, Philadelphia (31 $1^{\text {st }}$ Annual Meeting, November 7-10), as cited by Gelles 1980, pp. 880,884. Houghton 2021 explains that this was a panel discussion (speaking just before Gelles), from which her phrase is quoted, by which she intended "to critique the burgeoning belief in a myth/archetype [of] the batterer emerging from the [then] virtually nonexistent literature and the popular press."

Houghton BD (2021), Letter to the author, December 8 (re: "woozle effect"-cf. above and notes ${ }^{110,345}$ ).

Hu T-T, et al. (2016), "Assessment of the ABC/2 Method of Epidural Hematoma Volume Measurement as Compared to Computer-Assisted Planimetric Analysis," Biological Research for Nursing 18, 5-11, https://doi.org/10.1177/1099800415577634. Hungerford JP, et al. (2017), "Impact of ASPECT Scores and Infarct Distribution on Outcomes among Patients Undergoing Thrombectomy for Acute Ischemic Stroke with the Adapt Technique," Journal of Neurointerventional Surgery 9, 823-829, https://doi.org/10.1136/neurintsurg-2016-012528.

Huttner HB, et al. (2006), "Comparison of ABC/2 Estimation Technique to Computer-Assisted Planimetric Analysis in Warfarin-Related Intracerebral Parenchymal Hemorrhage," Stroke 37, 404-408,

https://doi.org/10.1161/01.STR.0000198806.67472.5c.

iSchemaView (n.d.), https://www.rapidai.com/rapid-aspects, last accessed 2021.12.12.

IST-3 Collaborative Group (2015), “Association Between Brain Imaging Signs, Early and Late Outcomes, and Response to Intravenous Alteplase after Acute Ischaemic Stroke in the Third International Stroke Trial (IST-3): Secondary Analysis of a Randomised Controlled Trial," Lancet Neurology 14,485-496, https://doi.org/10.1016/S1474-4422(15)00012-5.

Jacoby WG (1991), Data Theory and Dimensional Analysis, London: Sage Publications.

Jadhav AP, Wechsler LR (2016), “Patient Selection for Stroke Thrombectomy: Is CT Head Good Enough?” Neurology 87, 242-243, https://doi.org/10.1212/WNL.0000000000002872.

Jadhav AP, et al. (2017), "Correlation Between Clinical Outcomes and Baseline CT and CT Angiographic Findings in the SWIFT PRIME Trial," AJNR 38, 2270-2276, https://doi.org/10.3174/ainr.A5406. 
Jarcho JM, et al. (2011), “The Neural Basis of Rationalization: Cognitive Dissonance Reduction During Decision-making," Social Cognitive and Affective Neuroscience 6, 460-467, https://doi.org/10.1093/scan/nsq054.

Johnson SM, Bolstad OD (1972), "Methodological Issues in Naturalistic Observation: Some Problems and Solutions for Field Research, Final Report," https://files.eric.ed.gov/fulltext/ED071749.pdf, cited with original typescript page numbers. Joint Commission (2015), Disease-Specific Care Certification Program: Comprehensive Stroke Performance Measurement Implementation Guide, https://docplayer.net/18212597-Disease-specific-care-certification-program-comprehensive-stroke.html. Joint Commission (2018), email to the author from Sheryl Smith, Division of Healthcare Improvement, November 29. Jovin TG, et al. (2015), "Thrombectomy within 8 Hours after Symptom Onset in Ischemic Stroke," NEJM 372, 2296-2306, https://doi.org/10.1056/NEJMoa1503780.

Jovin TG (2019), via email as corresponding author of Streib et al. 2019, January 29.

Kaesmacher J, et al. (2019a), Letter, Stroke 50, e220-e221 (July), https://doi.org/10.1161/STROKEAHA.119.025857.

Kaesmacher J, et al. (2019b), "Mechanical Thrombectomy in Ischemic Stroke Patients With Alberta Stroke Program Early Computed Tomography Score 0-5," Stroke 50, 880-888, https://doi.org/10.1161/STROKEAHA.118.023465.

Kalafut MA, et al. (2000), "Detection of Early CT Signs of >1/3 Middle Cerebral Artery Infarctions: Interrater Reliability and Sensitivity of CT Interpretation by Physicians Involved in Acute Stroke Care," Stroke 31, 1667-1671, https://doi.org/10.1161/01.str.31.7.1667.

Kallmes DF, et al. (2019), Letter, Stroke 50, e219 (July), https://www.ahajournals.org/doi/10.1161/STROKEAHA.119.025741.

Kawano H, et al. (2010), "Presence of Deep White Matter Lesions on Diffusion-Weighted Imaging Is a Negative Predictor of Early Dramatic Improvement after Intravenous Tissue Plasminogen Activator Thrombolysis," Cerebrovascular Diseases 30, 230-236, https://doi.org/10.1159/000317183.

Kawano H, et al. (2012), "Modified ASPECTS for DWI Including Deep White Matter Lesions Predicts Subsequent Intracranial Hemorrhage," Journal of Neurology 259, 2045-2052, https://doi.org/10.1007/s00415-012-6446-1.

Kendall M (1952), The Advanced Theory of Statistics, vol. 1, $5^{\text {th }}$ ed., New York: Hafner.

Kendall M, Buckland WR (1957), A Dictionary of Statistical Terms, New York: Hafner.

Kenney JF (1940), Mathematics of Statistics, Part 2, London: Chapman \& Hall; available via archive.org.

Kenrose S (2016), "Point-Biserial Correlation \& Biserial Correlation: Definition, Examples," Statistics How To: Statistics for the Rest of Us, http://www.statisticshowto.com/point-biserial-correlation/, last accessed 2021.12.12.

Keynes JM (1921), A Treatise on Probability, London: Macmillan; available at https://www.gutenberg.org/ebooks/32625.

Khan M, et al. (2017), "Alberta Stroke Program Early CT Score Infarct Location Predicts Outcome Following M2 Occlusion," Frontiers in Neurology 8, Art. 98, https://doi.org/10.3389/fneur.2017.00098.

Kidwell CS, et al. (2013), “A Trial of Imaging Selection and Endovascular Treatment for Ischemic Stroke,” NEJM 368, 914-923, https://doi.org/10.1056/NEJMoa1212793.

Kidwell CS, Jahan R (2015), “Endovascular Treatment of Acute Ischemic Stroke,” Neurologic Clinics 33, 401-420, https://doi.org/10.1016/j.ncl.2015.01.005.

Kim EY, et al. (2016), "Comparison of Imaging Selection Criteria for Intra-Arterial Thrombectomy in Acute Ischemic Stroke with Advanced CT," European Radiology 26, 2974-2981, https://doi.org/10.1007/s00330-015-4141-1.

Kimura K, et al. (2008), "Large Ischemic Lesions on Diffusion-Weighted Imaging Done Before Intravenous Tissue Plasminogen Activator Thrombolysis Predicts a Poor Outcome in Patients With Acute Stroke," Stroke 39, 2388-2391, https://doi.org/10.1161/STROKEAHA.107.510917.

Kirkup L, Frenkel B (2006), An Introduction to Uncertainty in Measurement, Cambridge University Press.

Kleinman JT, et al. (2011), “ABC/2: Estimating Intracerebral Haemorrhage Volume and Total Brain Volume, and Predicting Outcome in Children,” Developmental Medicine and Child Neurology 53,281-284,

https://doi.org/10.1111/j.1469-8749.2010.03798.x.

Kobkitsuksakul C, et al. (2018), "Interobserver Agreement Between Senior Radiology Resident, Neuroradiology Fellow, and Experienced Neuroradiologist in the Rating of Alberta Stroke Program Early Computed Tomography Score (ASPECTS)," Diagnostic and Interventional Radiology 24, 104-107, https://doi.org/10.5152/dir.2018.17336.

Kosior RK, et al. (2010), "Atlas-Based Topographical Scoring for Magnetic Resonance Imaging of Acute Stroke," Stroke 41, 455-460, https://doi.org/10.1161/STROKEAHA.109.567289.

Kothari RU, et al. (1996), “The ABCs of Measuring Intracerebral Hemorrhage Volumes," Stroke 27, 1304-1305, https://doi.org/10.1161/01.STR.27.8.1304.

Kraemer HC (1979), "Ramifications of a Population Model for $\boldsymbol{\kappa}$ as a Coefficient of Reliability," Psychometrika 44, 461-472, https://doi.org/10.1007/BF02296208.

Krippendorff K vs. Fleiss JL (1978), "Reliability of Binary Attribute Data," Biometrics 34, 142-144; https://www.jstor.0og/stable/2529602. Krippendorff K (2004a), Content Analysis: An Introduction to its Methodology, $2^{\text {nd }}$ ed., Thousand Oaks CA: Sage Publications; this edition is cited because it is online at https://usu.instructure.com/files/70315935/download?download frd=1\&verifier= $\mathrm{kPCeVgRYVJ8UK2gEQNbehYHbiKYBNjWMFleh6j5G}$; citations are verified in $4^{\text {th }}$ ed. (Krippendorff 2019), converting cited pages as follows: $212 \rightarrow 278,213 \rightarrow 279,217-8 \rightarrow 283-4,221-56 \rightarrow 285-356,241-2 \rightarrow 356-8,244 \rightarrow 316,244-50 \rightarrow 315-23$. Krippendorff K (2004b), "Reliability in Content Analysis: Some Common Misconceptions and Recommendations," Human Communication Research 30, 411-433, https://doi.org/10.1111/i.1468-2958.2004.tb00738.x. 
Page 88 ASPECTS, The Mismeasure of Stroke: A Metrological Investigation

Krippendorff K (2019), Content Analysis: An Introduction to its Methodology, $4^{\text {th }}$ ed., Los Angeles CA: Sage Publications. Krishnan K, et al. (2015), "Performance Characteristics of Methods for Quantifying Spontaneous Intracerebral Haemorrhage: Data from the Efficacy of Nitric Oxide in Stroke (ENOS) Trial," JNNP 86, 1258-1266, https://doi.org/10.1136/innp-2014-309845. Kristjansson B, et al. (1996), "Optimal Cut-Points When Screening for More Than One Disease State: An Example from the Canadian Study of Health and Aging," J. Clin. Epidemiol. 49, 1423-1428, https://doi.org/10.1016/s0895-4356(96)00272-7. Krutch JW (1954), The Measure of Man, Indianapolis: Bobbs-Merrill.

Kuang H, et al. (2019), "Automated ASPECTS on Noncontrast CT Scans in Patients with Acute Ischemic Stroke Using Machine Learning," AJNR 40, 33-38, https://doi.org/10.3174/ajnr.A5889.

Kullback S (1987), "The Kullback-Leibler Distance" (letter), The American Statistician 41, 340-341.

Kwah LK, Diong J (2014), "National Institutes of Health Stroke Scale (NIHSS)," Journal of Physiotherapy 60, 61, https://doi.org/10.1016/j.jphys.2013.12.012.

Kwak R, et al. (1983), "Factors Affecting the Prognosis in Thalamic Hemorrhage," Stroke 14, 493-500

https://doi.org/10.1161/01.str.14.4.493.

Landis JR, Koch GG (1977), “The Measurement of Observer Agreement for Categorical Data," Biometrics 33, 159-174, https://doi.org/10.2307/2529310.

Langford E, et al. (2001), "Is the Property of Being Positively Correlated Transitive?" The American Statistician 55, 322-325, https://doi.org/10.1198/000313001753272286

Lansberg MG, et al. (2007), "Risk Factors of Symptomatic Intracerebral Hemorrhage After tPA Therapy for Acute Stroke," Stroke 38, 2275-2278, https://doi.org/10.1161/STROKEAHA.106.480475.

Lantz CA, Nebenzahl E (1996), "Behavior and Interpretation of the K Statistic: Resolution of the Two Paradoxes," Journal of Clinical Epidemiology 49, 431-434, https://doi.org/10.1016/0895-4356(95)00571-4.

Lapergue B, et al. (2017), "Effect of Endovascular Contact Aspiration vs Stent Retriever on Revascularization in Patients with Acute Ischemic Stroke and Large Vessel Occlusion: The ASTER Randomized Clinical Trial," JAMA 318, 443-452+Supp. https://doi.org/10.1001/jama.2017.9644.

Laredo C, et al. (2018), "Prognostic Significance of Infarct Size and Location: The Case of Insular Stroke," Scientific Reports 8, Art. 9498, https://doi.org/10.1038/s41598-018-27883-3.

Larrue V, et al. (2001), "Risk Factors for Severe Hemorrhagic Transformation in Ischemic Stroke Patients Treated With Recombinant Tissue Plasminogen Activator A Secondary Analysis of the European-Australasian Acute Stroke Study (ECASS II)," Stroke 32, 438-441, https://doi.org/10.1161/01.str.32.2.438.

Lassalle L. et al. (2016), "ASPECTS (Alberta Stroke Program Early CT Score) Assessment of the Perfusion-Diffusion Mismatch," Stroke 47, 2553-2558 https://doi.org/10.1161/STROKEAHA.116.013676.

Lee VH, et al. (2016), "Comparison of ABC/2 with the Simplified ABC/2 Formula in Calculating Intracerebral Hemorrhage Volume," Journal of Neurological Disorders \& Stroke 4(1), art. 1111,

https://www.jscimedcentral.com/NeurologicalDisorders/neurologicaldisorders-4-1111.pdf.

Lemaitre H, et al. (2012), "Normal age-related brain morphometric changes: nonuniformity across cortical thickness, surface area and gray matter volume?" Neurobiology of Aging 33, 617.e1-e9, https://doi.org/10.1016/j.neurobiolaging.2010.07.013.

Lemieux F, et al. (2012), "Insular Ischemic Stroke: Clinical Presentation and Outcome," Cerebrovascular Diseases Extra 2, 80-87+Supp., https://doi.org/10.1159/000343177.

Lesaffre E (2008), "Superiority, Equivalence, and Non-Inferiority Trials," Bulletin of the NYU Hospital for Joint Diseases 66,150-154, https://www.ncbi.nlm.nih.gov/pubmed?linkname=pubmed pubmed\&from uid=18537788.

Leslie-Mazwi TM (2019), "Invited Commentary on 'Imaging-based Selection for Endovascular Treatment in Stroke', Radiographics 39, 1714-1716, https://doi.org/10.1148/rg.2019190188.

Leslie-Mazwi TM, et al. (2019), "DEFUSE 3 Non-DAWN Patients A Closer Look at Late Window Thrombectomy Selection," Stroke 50, 618-625, https://doi.org/10.1161/STROKEAHA.118.023310.

Liang BA, Zivin JA (2008), "Empirical Characteristics of Litigation Involving Tissue Plasminogen Activator and Ischemic Stroke," Annals of Emergency Medicine 52, 160-164, https://doi.org/10.1016/j.annemergmed.2007.12.017.

Liebeskind DS, et al. (2014), "Serial Alberta Stroke Program Early CT Score from Baseline to 24 Hours in Solitaire Flow Restoration With the Intention for Thrombectomy Study: A Novel Surrogate End Point for Revascularization in Acute Stroke," Stroke 45, 723-727, https://doi.org/10.1161/STROKEAHA.113.003914.

Liebeskind DS, et al. (2021), "Serial ASPECTS in the DAWN Trial: Infarct Evolution and Clinical Impact," Stroke 52, 3318-3324, https://doi.org/10.1161/STROKEAHA.120.033477.

Liebetrau AM (1983), Measures of Association, Beverly Hills CA: Sage University Paper 32.

Light RJ (1971), "Measures of Response Agreement for Qualitative Data: Some Generalizations and Alternatives," Psychological Bulletin 76, 365-377, https://doi.org/10.1037/h0031643.

Lin C-J, Saver JL (2019), "Noninferiority Margins in Trials of Thrombectomy Devices for Acute Ischemic Stroke: Is the Bar Being Set Too Low?" Stroke 50, 3519-3526, https://doi.org/10.1161/STROKEAHA.119.026717.

Lin K, et al. (2008), "Accuracy of the Alberta Stroke Program Early CT Score during the First 3 Hours of Middle Cerebral Artery Stroke: Comparison of Noncontrast CT, CT Angiography Source Images, and CT Perfusion,” AJNR 29, 931-936, https://doi.org/10.3174/ainr.A0975. 
Lin K, et al. (2009), "Correlation of Volumetric Mismatch and Mismatch of Alberta Stroke Program Early CT Scores on CT Perfusion Maps," Neuroradiology 51, 17-23, https://doi.org/10.1007/s00234-008-0454-y.

Lin K, et al. (2011), "What ASPECTS Value Best Predicts the 100-mL Threshold on Diffusion Weighted Imaging? Study of 150 Patients with Middle Cerebral Artery Stroke," Journal of Neuroimaging 21, 229-231, https://doi.org/10.1111/j.1552-6569.2010.00487.x.

Lin K (2018), via email to the author, November 13.

Lisk DR, et al. (1994), "Early Presentation of Hemispheric Intracerebral Hemorrhage: Prediction of Outcome and Guidelines for Treatment Allocation," Neurology 44, 133-139, https://doi.org/10.1212/wnl.44.1.133.

Liu L, et al. (2017), "Computed Tomography Perfusion Alberta Stroke Program Early Computed Tomography Score Is Associated with Hemorrhagic Transformation after Acute Cardioembolic Stroke," Frontiers in Neurology 8, Art. 591, https://doi.org/10.3389/fneur.2017.00591.

Lo C (2017), "e-ASPECTS: Accelerating the Stroke Pathway," London UK: Verdict Medical Devices, https://www.medicaldevice-network.com/features/featuree-aspects-accelerating-the-stroke-pathway-5888411/, last accessed 2021.12.12. Logan C, et al. (2018), "Borderline Alberta Stroke Programme Early CT Score Patients with Acute Ischemic Stroke Due to Large Vessel Occlusion May Find Benefit with Endovascular Thrombectomy," World Neurology 110, e653-e658, https://doi.org/10.1016/j.wneu.2017.11.068.

Loh Y, et al. (2009), "Basal Ganglionic Infarction Before Mechanical Thrombectomy Predicts Poor Outcome," Stroke 40, 3315-3320, https://doi.org/10.1161/STROKEAHA.109.551705.

Lou M, et al. (2008), "The HAT Score: A Simple Grading Scale for Predicting Hemorrhage After Thrombolysis," Neurology 71, 1417-1423, https://doi.org/10.1212/01.wnl.0000330297.58334.dd.

Lövblad K-O, et al. (1997), "Ischemic Lesion Volumes in Acute Stroke by Diffusion-Weighted Magnetic Resonance Imaging Correlate with Clinical Outcome," Annals of Neurology 42, 164-170, https://doi.org/10.1002/ana.410420206. Luby M, et al. (2013), "Stroke Mismatch Volume with the Use of ABC/2 Is Equivalent to Planimetric Stroke Mismatch Volume," AJNR 34, 1901-1907, https://doi.org/10.3174/ajnr.A3476.

Lum C, et al. (2014), "Computed Tomographic Angiography and Cerebral Blood Volume Can Predict Final Infarct Volume and Outcome After Recanalization," Stroke 45, 2683-2688, https://doi.org/10.1161/STROKEAHA.114.006163. Luvizutto GJ, et al. (2015), "ASPECTS Correlates with Scandinavian Stroke Scale for Predicting Early Neurological Impairment," Arquivos de Neuro-Psiquiatria 73, 450-453, https://doi.org/10.1590/0004-282X20150037.

Lyden PD, et al. (2001), "A Modified National Institutes of Health Stroke Scale for Use in Stroke Clinical Trials: Preliminary Reliability and Validity," Stroke 32, 1310-1317, https://doi.org/10.1161/01.str.32.6.1310.

Lyden PD, et al. (2004), "Factor Analysis of the National Institutes of Health Stroke Scale in Patients with Large Strokes," Archives of Neurology 61, 1677-1680, https://doi.org/10.1001/archneur.61.11.1677.

Lyden PD (2019), “Thrombolytic Therapy for Acute Ischemic Stroke: A Very Great Honor," Stroke 50, 2597-2603, https://doi.org/10.1161/STROKEAHA.119.025699.

Ma H, et al. (2019), “Thrombolysis Guided by Perfusion Imaging up to 9 Hours after Onset of Stroke," NEJM 380, 1795-1803, https://doi.org/10.1056/NEJMoa1813046.

Mac Cormac ER (1985), A Cognitive Theory of Metaphor, Cambridge MA: The MIT Press.

Macdonald-Ross M(1977), "How Numbers Are Shown: A Review of Research on the Presentation of Quantitative Data," AV Communication Review 25, 359-409.

MacKay DM (1953), “The Nomenclature of Information Theory,” Trans IRE Professional Group on Information Theory 1, 9-21, https://doi.org/10.1109/TIT.1953.1188575.

Maclure M, Willett WC (1987), "Misinterpretation and Misuse of the Kappa Statistic," Amer JEpidemiology 126, 161-169, https://doi.org/10.1093/aje/126.2.161.

Maegerlein C, et al. (2019), "Automated Calculation of the Alberta Stroke Program Early CT Score: Feasibility and Reliability," Radiology 291, 141-148, https://doi.org/10.1148/radiol.2019181228.

Mak HKF, et al. (2003), "Hypodensity of >1/3 Middle Cerebral Artery Territory Versus Alberta Stroke Programme Early CT Score (ASPECTS): Comparison in Hyperacute Ischemic Stroke in the Community Setting," Stroke 34, 1194-1196, https://doi.org/10.1161/01.STR.0000069162.64966.71.

Makris N, Pandya DN (2009), "The Extreme Capsule in Humans and Rethinking of the Language Circuitry," Brain Structure and Function 213, 343-358, https://doi.org/10.1007/s00429-008-0199-8.

Maradi V, et al. (2019), "Measurement of Cerebral Infarct Volume by Magnetic Resonance Imaging using ABC/2 Method and its Correlation with Clinical Outcome using NIHSS Score," International Journal of Contemporary Medicine Surgery and Radiology 4(2), B144-B149, http://dx.doi.org/10.21276/ijcmsr.2019.4.2.32.

Marks MP et al. (1999), "Evaluation of Early Computed Tomographic Findings in Acute Ischemic Stroke," Stroke 30, 389-392, https://doi.org/10.1161/01.str.30.2.389.

Marler JR, et al. [NINDS] (1995) “Tissue Plasminogen Activator for Acute Ischemic Stroke," NEJM 333, 1581-1587, https://doi.org/10.1056/NEJM199512143332401. [Overall responsibility for manuscript is stated in p. 1581 footnote.] 
Page 90 ASPECTS, The Mismeasure of Stroke: A Metrological Investigation

Marnat G, et al. (2019), "First-Line Sofia Aspiration Thrombectomy Approach within the Endovascular Treatment of Ischemic Stroke Multicentric Registry: Efficacy, Safety, and Predictive Factors of Success," AJNR 40, 1006-1012 + E1-6, https://doi.org/10.3174/ainr.A6074.

Marsh EB, et al. (2013), "Hemorrhagic Transformation in Patients with Acute Ischaemic Stroke and an Indication for Anticoagulation,” European Journal of Neurology 20, 962-967, https://doi.org/10.1111/ene.12126.

Marsh EB, et al. (2016), "Predicting Hemorrhagic Transformation of Acute Ischemic Stroke: Prospective Validation of the HeRS Score," Medicine 95, 1-8, https://doi.org/10.1097/MD.0000000000002430.

Mathur BN (2014), "The Claustrum in Review," Frontiers in Systems Neurosci 8, Art.48, https://doi.org/10.3389/fnsys.2014.00048. Mauri L, D'Agostino RB (2017), "Challenges in the Design and Interpretatiion of Noninferiority Trials," NEJM 377, 1357-1367, https://doi.org/10.1056/NEJMra1510063.

McTaggart RA, et al. (2015), "Alberta Stroke Program Early Computed Tomographic Scoring Performance in a Series of Patients Undergoing Computed Tomography and MRI: Reader Agreement, Modality Agreement, and Outcome Prediction," Stroke 46, 407-412, https://doi.org/10.1161/STROKEAHA.114.006564.

Meadowlark W (1963), "A la recherche du Pooh perdu," in Crews FC, ed., The Pooh Perplex: A Freshman Casebook, New York: Dutton; paperback, Dutton, 1965, pp. 74-85.

Meillet A (1912), "L’Évolution des Formes Grammaticales," Scientia (Revista di scienza) 12, 130-158.

Mendelow AD, et al. (2005), "Early surgery versus initial conservative treatment in patients with spontaneous supratentorial intracerebral haematomas in the International Surgical Trial in Intracerebral Haemorrhage (STICH): a randomised trial," Lancet 365, 387-397, https://www.thelancet.com/article/S0140-6736(05)17826-X/fulltext.

Mendelow AD, et al. (2013), "Early surgery versus initial conservative treatment in patients with spontaneous supratentorial lobar intracerebral haematomas (STICH II): a randomised trial," Lancet 382, 397-408,

http://dx.doi.org/10.1016/S0140-6736(13)60986-1.

Menezes NM, et al. (2007), "The Real Estate Factor: Quantifying the Impact of Infarct Location on Stroke Severity," Stroke 38, 194-197, https://doi.org/10.1161/01.STR.0000251792.76080.45.

Menon BK, et al. (2011), "ASPECTS and Other Neuroimaging Scores in the Triage and Prediction of Outcome in Acute Stroke Patients," Neuroimaging Clinics of North America 21:2 (May, Stroke Imaging), 407-423, https://doi.org/10.1016/i.nic.2011.01.007.

Menon BK, et al. (2015), "Role of Imaging in Current Acute Ischemic Stroke Workflow for Endovascular Therapy," Stroke 46, 1453-1461, https://doi.org/10.1161/STROKEAHA.115.009160.

Menon BK, Goyal M (2015), "Imaging Paradigms in Acute Ischemic Stroke: A Pragmatic Evidence-based Approach," Radiology 277, 7-12, https://doi.org/10.1148/radiol.2015151030.

Messé SR, et al. (2007), “CT-NIHSS Mismatch Does Not Correlate With MRI Diffusion-Perfusion Mismatch," Stroke 38, 2079-2084, https://doi.org/10.1161/STROKEAHA.106.480731.

Meyer BC, et al. (2002), "Modified National Institutes of Health Stroke Scale for Use in Stroke Clinical Trials: Prospective Reliability and Validity," Stroke 33, 1261-1266, https://doi.org/10.1161/01.str.0000015625.87603.a7.

Michel P, et al. (2012), "Perfusion-CT Guided Intravenous Thrombolysis in Patients with Unknown-onset Stroke: A Randomized, Double-blind, Placebo-controlled, Pilot Feasibility Trial," Neuroradiology 54, 579-588,

https://doi.org/10.1007/s00234-011-0944-1.

Miller \& Zois, LLC (2018) “Failure to Give tPA Stroke Lawsuit,” https://www.millerandzois.com/tpa-stroke-malpractice-lawsuit.html. Millis SR, et al. (2007), "Measurement Properties of the National Institutes of Health Stroke Scale for People with Rightand Left-Hemisphere Lesions: Further Analysis of the Clomethiazole for Acute Stroke Study-Ischemic (Class-I) Trial," Archives of Physical Medicine and Rehabilitation 88, 302-308, https://doi.org/10.1016/j.apmr.2006.12.027.

Milne AA(1926), "Chapter Three in which Pooh and Piglet go hunting and nearly catch a Woozle," in Winnie-the-Pooh, London: Methuen, Toronto: McClelland \& Stewart, New York: Dutton; reprinted many times with varying pagination; available at https://www.e-reading.club/chapter.php/71304/4/Milne - Winnie-The-Pooh and All\%2C All\%2C All.html. Mitomi M, et al. (2014), "Comparison of CT and DWI Findings in Ischemic Stroke Patients within 3 Hours of Onset," JSCD 23, 37-42, https://doi.org/10.1016/i.jstrokecerebrovasdis.2012.08.014.

Mocco J, et al. (2016), "Aspiration Thrombectomy After Intravenous Alteplase Versus Intravenous Alteplase Alone," Stroke 47, 2331-2338, https://doi.org/10.1161/STROKEAHA.116.013372.

Modi J, et al. (2012), "Enhancing Acute Ischemic Stroke Interpretation with Online ASPECTS Training," The Canadian Journal of Neurological Sciences 39(1), 112-114 (letter), https://doi.org/10.1017/S0317167100012841.

Mokin M, et al. (2017), "ASPECTS (Alberta Stroke Program Early CT Score) Measurement Using Hounsfield Unit Values When Selecting Patients for Stroke Thrombectomy," Stroke 48, 1574-1579, https://doi.org/10.1161/STROKEAHA.117.016745. Molina CA, et al. (2004), "Improving the Predictive Accuracy of Recanalization on Stroke Outcome in Patients Treated With Tissue Plasminogen Activator," Stroke 35, 151-157, https://doi.org/10.1161/01.STR.0000106485.04500.4A.

Montesquieu [de Secondat C-L] (1725-1755), Mes Pensées (notes published posthumously), e.g.: Pensées et Fragments Inédits de Montesquieu, Bordeaux: Gounouilhou, 1899, available via archive.org.

Mosteller F, Tukey JW (1977), Data Analysis and Regression, Addison-Wesley, p. 89.

Mourand I, et al. (2018), "Favorable Revascularization Therapy in Patients with ASPECTS $\leq 5$ on DWI in Anterior Circulation Stroke," Journal of Neurointerventional Surgery 10, 5-9, https://doi.org/10.1136/neurintsurg-2017-013358. 
Muir KW, et al. (1996), "Comparison of Neurological Scales and Scoring Systems for Acute Stroke Prognosis,” Stroke 27, 1817-1820, https://doi.org/10.1161/01.str.27.10.1817.

Muir KW, et al. (2017), "Endovascular Therapy for Acute Ischaemic Stroke: The Pragmatic Ischaemic Stroke Thrombectomy Evaluation (PISTE) Randomised, Controlled Trial," JNNP 88, 38-44, https://doi.org/10.1136/jnnp-2016-314117.

Na DG, et al. (2005), "CT Sign of Brain Swelling without Concomitant Parenchymal Hypoattenuation: Comparison with Diffusion and Perfusion-weighted MR Imaging,' Radiology 235, 992-998, https://doi.org/10.1148/radiol.2353040571.

Nabavi DG, et al. (2002), "MOSAIC: Multimodal Stroke Assessment Using Computed Tomography: Novel Diagnostic Approach for the Prediction of Infarction Size and Clinical Outcome,” Stroke 33, 2819-2826, https://doi.org/10.1161/01.str.0000043074.39077.60.

Nael K, et al. (2019), “Imaging-based Selection for Endovascular Treatment in Stroke,” Radiographics 39, 1696-1713, https://doi.org/10.1148/rg.2019190030.

Nagel S, et al. (2017), “e-ASPECTS Software Is Non-inferior to Neuroradiologists in Applying the ASPECT Score to Computed Tomography Scans of Acute Ischemic Stroke Patients," International Journal of Stroke 12, 615-622, https://doi.org/10.1177/1747493016681020.

Nagel S, et al. (2018), "Clinical Utility of Electronic Alberta Stroke Program Early Computed Tomography Score Software in the ENCHANTED Trial Database," Stroke 49, 1407-1411, https://doi.org/10.1161/STROKEAHA.117.019863.

Nannoni S, et al. (2021), "Correlation Between ASPECTS and Core Volume on CT Perfusion: Impact of Time Since Stroke Onset and Presence of Large-Vessel Occlusion," AJNR 42, 422-428; https://doi.org/10.3174/ajnr.A6959.

Naylor J, et al. (2017), "Reliability and Utility of the Alberta Stroke Program Early Computed Tomography Score in Hyperacute Stroke,' JSCD 26, 2547-2552, https://doi.org/10.1016/i.jstrokecerebrovasdis.2017.05.042.

Nelson RF, et al. (1980), “Computed Tomography in Patients Presenting with Lacunar Syndromes,” Stroke 11, 256-261, https://doi.org/10.1161/01.str.11.3.256.

Neugebauer O (1957/1969), The Exact Sciences in Antiquity, $2^{\text {nd }}$ ed., Providence RI: Brown University; 1957; reprinted slightly corrected, New York: Dover, 1969.

Newman GC (2007), “Clarification of abc/2 Rule for ICH Volume,” Stroke 38, 862, https://doi.org/10.1161/01.STR.0000257309.50643.0a. Nezu T, et al. (2011), "Early Ischemic Change on CT Versus Diffusion-Weighted Imaging for Patients With Stroke Receiving Intravenous Recombinant Tissue-Type Plasminogen Activator Therapy: Stroke Acute Management with Urgent Risk-factor Assessment and Improvement (SAMURAI) rt-PA Registry," Stroke 42, 2196-2200,

https://doi.org/10.1161/STROKEAHA.111.614404.

Nicholas JV, White DR (2001), Traceable Temperatures, $2^{\text {nd }}$ ed., Chichester UK: Wiley. Chapter 1 (cited) is available at http://www.isotech.info/pdfs/tracechap1.pdf.

Nicholson P, et al. (2020), "Per-Region Interobserver Agreement of Alberta Stroke Program Early CT Scores (ASPECTS)," Journal of Neurointerventional Surgery 12, 1069-1071; https://doi.org/10.1136/neurintsurg-2019-015473 (paginated 1-4). NINDS t-PA Stroke Study Group (1997), "Intracerebral Hemorrhage after t-PA Therapy for Ischemic Stroke," Stroke 28, 2109-2118, https://doi.org/10.1161/01.str.28.11.2109.

Nishi H, et al. (2019), "Predicting Clinical Outcomes of Large Vessel Occlusion Before Mechanical Thrombectomy Using Machine Learning," Stroke 50, 2379-2388, http://doi.org/10.1161/STROKEAHA.119.025411.

Nogueira RG, et al. (2018a), "Thrombectomy 6 to 24 Hours after Stroke with a Mismatch between Deficit and Infarct," NEJM 378, 11-21 + Supplementary Appendix, https://doi.org/10.1056/NEJMoa1706442.

Nogueira RG, et al. (2018b), "Safety and Efficacy of a 3-Dimensional Stent Retriever With Aspiration-Based Thrombectomy vs Aspiration-Based Thrombectomy Alone in Acute Ischemic Stroke Intervention: A Randomized Clinical Trial," JAMA Neurology 75, 304-311 + Supplements, https://doi.org/10.1001/jamaneurol.2017.3967.

Nogueira RG, Ribó M (2019), “Endovascular Treatment of Acute Stroke A Call for Individualized Patient Selection,” Stroke 50, 2612-2618 + Supplemental Material, https://doi.org/10.1161/STROKEAHA.119.023811.

Nolan JR, et al. (1990), Black's Law Dictionary, St. Paul: West.

Noorian AR, et al. (2015), "Endovascular Therapy in Strokes with ASPECTS 5-7 May Result in Smaller Infarcts and Better Outcomes as Compared to Medical Treatment Alone,' Interventional Neurology 4, 30-37, https://doi.org/10.1159/000438775. Ntaios G, et al. (2012), "An integer-based score to predict functional outcome in acute ischemic stroke: The ASTRAL score," Neurology 78, 1916-1922, https://doi.org/10.1212/WNL.0b013e318259e221.

Oberg J (1999), “Why the Mars Probe Went OffCourse,' IEEE Spectrum 36:12 (Dec.), 34-39, https://doi.org/10.1109/6.809121, https://spectrum.ieee.org/aerospace/robotic-exploration/why-the-mars-probe-went-off-course.

Olivé-Gadea M, et al. (2019), "Baseline ASPECTS and e-ASPECTS Correlation with Infarct Volume and Functional Outcome in Patients Undergoing Mechanical Thrombectomy," JNeuroimaging 29, 198-202, https://doi.org/10.1111/jon.12564. Olivot J-M, et al. (2013), "Impact of Diffusion-Weighted Imaging Lesion Volume on the Success of Endovascular Reperfusion Therapy,” Stroke 44, 2205-2211, https://doi.org/10.1161/STROKEAHA.113.000911.

Opitz E, Schneider M (1950), "Über die Sauerstoffversorgung des Gehirns und den Mechanismus der Mangelwirkungen," Ergebnisse der Physiologie, biologischen Chemie und experimentellen Pharmakologie 46, 126-260, cited by Hossmann 1994. Oppenheim C, et al. (2000), "Prediction of Malignant Middle Cerebral Artery Infarction by Diffusion-Weighted Imaging," Stroke 31, 2175-2181, https://doi.org/10.1161/01.str.31.9.2175. 
Page 92 ASPECTS, The Mismeasure of Stroke: A Metrological Investigation

Orwell G (1949), 1984, London: Secker \& Warburg.

Osborn AG (1994), Diagnostic Neuroradiology, St. Louis: Mosby.

Ospel JM, et al. (2021a), "Radiologic Patterns of Intracranial Hemorrhage and Clinical Outcome after Endovascular Treatment in Acute Ischemic Stroke: Results from the ESCAPE-NA1 Trial," Radiology 300, 402-409, https://doi.org/10.1148/radiol.2021204560.

Ospel JM, et al. (2021b), "Clinical Outcomes of Isolated Deep Grey Matter Infarcts after Endovascular Treatment of Large Vessel Occlusion Stroke," Neuroradiology 63,1463-1469, https://doi.org/10.1007/s00234-021-02656-4.

Ospel JM, et al. (2021c), "A Detailed Analysis of Infarct Patterns and Volumes at 24-hour Noncontrast CT and Diffusion-weighted MRI in Acute Ischemic Stroke Due to Large Vessel Occlusion: Results from the ESCAPE-NA1 Trial," Radiology 300, 152-159+supp., https://doi.org/10.1148/radiol.2021203964.

O'Toole G (2014), "History Does Not Repeat Itself, But It Rhymes," https://quoteinvestigator.com/2014/01/12/history-rhymes/. Padroni M, et al. (2016), "Cerebral Blood Volume ASPECTS Is the Best Predictor of Clinical Outcome in Acute Ischemic Stroke: A Retrospective, Combined Semi-Quantitative and Quantitative Assessment," PLoS ONE 11(1), e0147910, https://doi.org/10.1371/journal.pone.0147910.

Painsky A, Wornell G(2018), “On the Universality of the Logistic Loss Function,” arXiv:1805.03804v1 [cs.IT] 10 May 2018, https://arxiv.org/pdf/1805.03804.pdf.

Panni P, et al. (2019), “Acute Stroke With Large Ischemic Core Treated by Thrombectomy: Predictors of Good Outcome and Mortality," Stroke 50, 1164-1171, https://doi.org/10.1161/STROKEAHA.118.024295.

Pantano P, et al. (1999), "Delayed Increase in Infarct Volume After Cerebral Ischemia Correlations with Thrombolytic Treatment and Clinical Outcome," Stroke 30, 502-507, https://doi.org/10.1161/01.str.30.3.502.

Parsons MW, et al. (2005), "Perfusion Computed Tomography: Prediction of Final Infarct Extent and Stroke Outcome," Annals of Neurology 58, 672-679, https://doi.org/10.1002/ana.20638.

Patel SC, et al. (2001), "Lack of Clinical Significance of Early Ischemic Changes on Computed Tomography in Acute Stroke," JAMA 286, 2830-2838, https://doi.org/10.1001/jama.286.22.2830.

Pavitt C (2017), "Reliability, Unitizing," in Allen M, ed., The SAGE Encyclopedia of Communication Research Methods, Thousand Oaks CA: SAGE Publications, vol. 3, pp. 1421-1424.

Payabvash S, et al. (2017), "Acute Ischemic Stroke Infarct Topology: Association with Lesion Volume and Severity of Symptoms at Admission and Discharge," AJNR 38, 58-63, https://doi.org/10.3174/ajnr.A4970.

Payabvash S, et al. (2018), "Multivariate Prognostic Model of Acute Stroke Combining Admission Infarct Location and Symptom Severity: A Proof-of-Concept Study," Journal of Stroke and Cerebrovascular Diseases 27, 936-944, https://doi.org/10.1016/i.jstrokecerebrovasdis.2017.10.034.

Pearson CE (1983), Handbook of Applied Mathematics: Selected Results and Methods, $2^{\text {nd }}$ ed., New York: Van Nostrand. Pearson K (1895), "Note on Regression and Inheritance in the Case of Two Parents," Proceedings of the Royal Society of London 58, 240-242, https://doi.org/10.1098/rspl.1895.0041.

Pearson K (1896), "Mathematical Contributions to the Theory of Evolution. III. Regression, Heredity, and Panmixia," Philosophical Transactions of the Royal Society of London. Series A, Mathematical or Physical 187, 253-318, https://doi.org/10.1098/rsta.1896.0007.

Pearson K (1901), "On Lines and Planes of Closest Fit to Systems of Points in Space," The London, Edinburgh and Dublin Philosophical Magazine and Journal of Science, Sixth Series, 2, 559-572, https://doi.org/10.1080/14786440109462720.

Pearson K (1920), "Notes on the History of Correlation," Biometrika 13, 25-45, https://doi.org/10.1093/biomet/13.1.25.

Pedraza S, et al. (2012), "Reliability of the $\mathrm{ABC} / 2$ Method in Determining Acute Infarct Volume," Journal of Neuroimaging 22, 155-159, https://doi.org/10.1111/j.1552-6569.2011.00588.x.

Pexman JHW, et al. (2001), "Use of the Alberta Stroke Program Early CT Score (ASPECTS) for Assessing CT Scans in Patients with Acute Stroke," AJNR 22, 1534-1542, http://www.ainr.org/content/22/8/1534 and

https://www.ncbi.nlm.nih.gov/pubmed?linkname=pubmed pubmed\&from uid=11559501.

Pfaff J, et al. (2017), "e-ASPECTS Correlates with and Is Predictive of Outcome after Mechanical Thrombectomy," AJNR 38, 1594-1599, https://doi.org/10.3174/ajnr.A5236.

Phan K, et al. (2019), "Influence of ASPECTS and Endovascular Thrombectomy in Acute Ischemic Stroke: a MetaAnalysis," Journal of Neurointerventional Surgery 11, 664-669, https://doi.org/10.1136/neurintsurg-2018-014250.

Phan TG, et al. (2005), "A Digital Map of Middle Cerebral Artery Infarcts Associated with Middle Cerebral Artery Trunk and Branch Occlusion," Stroke 36, 986-991, https://doi.org/10.1161/01.STR.0000163087.66828.e9.

Phan TG, et al. (2006), "The ASPECTS Template Is Weighted in Favor of the Striatocapsular Region," NeuroImage 31, 477-481, https://doi.org/10.1016/j.neuroimage.2005.12.059.

Phan TG, et al. (2010), "Development of a New Tool to Correlate Stroke Outcome with Infarct Topography: A Proof-ofConcept Study," NeuroImage 49, 127-133, https://doi.org/10.1016/i.neuroimage.2009.07.067.

Phan TG, et al. (2013), "Proof of Concept Study: Relating Infarct Location to Stroke Disability in the NINDS rt-PA Trial," Cerebrovascular Diseases 35, 560-565, https://doi.org/10.1159/000351147.

Piaget J (1941/1952), La Genèse du Nombre chez l'Enfant, Paris: Delachaux \& Niestlé, 1941; Gattegno C, Hodgson FM, trans., The Child's Conception of Number, London: Routledge and Kegan Paul, 1952; reprinted, New York, Norton, 1965. 
Plato (c. 367 BCE), “Theaetetus,” in Plato, vol. VII, trans. by Fowler HN, Loeb Classical Library, Harvard University Press, 1921, 3-257.

Potter CA, et al. (2019), "CT for Treatment Selection in Acute Ischemic Stroke: A Code Stroke Primer," Radiographics 39, 1717-1738, https://doi.org/10.1148/rg.2019190142.

Powers WJ, et al. (2015), "2015 American Heart Association/American Stroke Association Focused Update of the 2013 Guidelines for the Early Management of Patients With Acute Ischemic Stroke Regarding Endovascular Treatment: A Guideline for Healthcare Professionals From the American Heart Association/American Stroke Association," Stroke 46, 3020-35, https://doi.org/10.1161/STR.0000000000000074.

Powers WJ, et al. (2018), "2018 Guidelines for the Early Management of Patients With Acute Ischemic Stroke: A Guideline for Healthcare Professionals From the American Heart Association / American Stroke Association," Stroke 49, e46-e99, https://doi.org/10.1161/STR.0000000000000158

Powers WJ, et al. (2019), "Guidelines for the Early Management of Patients With Acute Ischemic Stroke: 2019 Update to the 2018 Guidelines for the Early Management of Acute Ischemic Stroke: A Guideline for Healthcare Professionals From the American Heart Association/American Stroke Association," Stroke 50, e344-e418+Supplements, https://doi.org/10.1161/STR.0000000000000211.

Puetz V, et al. (2008), "Extent of Hypoattenuation on CT Angiography Source Images Predicts Functional Outcome in Patients With Basilar Artery Occlusion," Stroke 39, 2485-2490, https://doi.org/10.1161/STROKEAHA.107.511162.

Puetz V, et al. (2009), "The Alberta Stroke Program Early CT Score in Clinical Practice: What Have We Learned?" International Journal of Stroke 4, 354-364, https://doi.org/10.1111/j.1747-4949.2009.00337.x.

Puig J, et al. (2011), "Acute Damage to the Posterior Limb of the Internal Capsule on Diffusion Tensor Tractography as an Early Imaging Predictor of Motor Outcome after Stroke," AJNR 32, 857-863, https://doi.org/10.3174/ajnr.A2400. Pushkin AS (1833/1964), Eugene Onegin (Евгений Онъгинъ), St. Petersburg: Alexander Smirdin, 1833; $2^{\text {nd }}$ ed. (1837) trans. by Nabokov VV, New York: Bollingen Series LXXII, 1964.

Qiu W, et al. (2020), "Machine Learning for Detecting Early Infarction in Acute Stroke with Non-Contrast-enhanced CT," Radiology 294, 638-644, https://doi.org/10.1148/radiol.2020191193.

Quintilian (c. 95), The Institutio Oratoria of Quintilian, III, trans. by Butler HE, Cambridge MA: Loeb Classical Library, Harvard University Press, 1921; available at books.google.com.

Quote Investigator (2015), "Suppose You Call a Sheep’s Tail a Leg, How Many Legs Will the Sheep Have? https://quoteinvestigator.com/2015/11/15/legs/, last accessed 2021.12.12.

Rangaraju S, et al. (2015), "Relationship Between Lesion Topology and Clinical Outcome in Anterior Circulation Large Vessel Occlusions," Stroke 46, 1787-1792, https://doi.org/10.1161/STROKEAHA.115.009908.

Reidler P, et al. (2019), "Attenuation Changes in ASPECTS Regions: A Surrogate for CT Perfusion-based Ischemic Core in Acute Ischemic Stroke," Radiology 291, 451-458 + Appendix E1, https://doi.org/10.1148/radiol.2019182041.

Ringleb P, et al. (2019), "Extending the Time Window for Intravenous Thrombolysis in Acute Ischemic Stroke Using Magnetic Resonance Imaging-Based Patient Selection," International Journal of Stroke 14, 483-490,

https://doi.org/10.1177/1747493019840938.

Roberts HC, et al. (2002), "Computed Tomographic Findings in Patients Undergoing Intra-arterial Thrombolysis for Acute Ischemic Stroke Due to Middle Cerebral Artery Occlusion: Results From the PROACT II Trial," Stroke 33, 15571567, https://doi.org/10.1161/01.STR.0000018011.66817.41.

Ros Mendoza LH, et al. (2009), "Estudio Anátomo-radiológico Mediante Segmentación de los Núcleos Cerebrales Caudado, Putamen y Pálido," Revista Argentina de Radiología 73(4), 457-464; https://www.redalyc.org/pdf/3825/382538478012.pdf.

Ross KA (1980), Elementary Analysis: The Theory of Calculus, New York: Springer-Verlag.

Rosso C, et al. (2019), "Impact of Infarct Location on Functional Outcome Following Endovascular Therapy for Stroke," JNNP 90, 313-319, http://doi.org/10.1136/innp-2018-318869.

Rothstein J (1951), “Information, Measurement, and Quantum Mechanics," Science 114, 171-175, https://doi.org/10.1126/science.114.2955.171.

Rothwell PM, et al. (2005), "A Simple Score (ABCD) to Identify Individuals at High Early Risk of Stroke after Transient Ischaemic Attack," The Lancet 366, 29-36, https://doi.org/10.1016/S0140-6736(05)66702-5.

Rothwell PM, Buchan AM (2012), "Mobile Acute Stroke Units: Bringing the Hospital to the Patient," Lancet Neurology 11, 382-383, https://doi.org/10.1016/S1474-4422(12)70069-8.

Russell B (1903), Principles of Mathematics, volume 1, Cambridge University Press; reprinted, 1938, unchanged except for deletion of "volume 1" and addition of an "Introduction to the Second Edition"; reprinted, 1996, New York: Norton. Sallustio F, et al. (2017), "CT Angiography ASPECTS Predicts Outcome Much Better Than Noncontrast CT in Patients with Stroke Treated Endovascularly," AJNR 38, 1569-1573, https://doi.org/10.3174/ajnr.A5264.

Šańák D, et al. (2006), "Impact of Diffusion-weighted MRI-measured Initial Cerebral Infarction Volume on Clinical Outcome in Acute Stroke Patients with Middle Cerebral Artery Occlusion Treated by Thrombolysis," Neuroradiology 48, 632-639, https://doi.org/10.1007/s00234-006-0105-0. 
Page 94 ASPECTS, The Mismeasure of Stroke: A Metrological Investigation

San Román L, et al. (2018), "Imaging Features and Safety and Efficacy of Endovascular Stroke Treatment: a Meta-analysis of Individual Patient-level Data," Lancet Neurology 17, 895-904, https://doi.org/10.1016/S1474-4422(18)30242-4.

Saposnik G (2004), "Predicting Functional Outcome After Intra-Arterial Thrombolysis: Aspects of ASPECTS," Stroke 35(1), e7-e8, https://doi.org/10.1161/01.STR.0000107771.06671.7E.

Saposnik G, et al. (2011), "IScore: A Risk Score to Predict Death Early After Hospitalization for an Acute Ischemic Stroke," Circulation 123, 739-749, https://doi.org/10.1161/CIRCULATIONAHA.110.983353.

Saposnik G, et al. (2019), "Factors Associated With the Decision-Making on Endovascular Thrombectomy for the Management of Acute Ischemic Stroke," Stroke 50, 2441-2447+Supp., https://doi.org/10.1161/STROKEAHA.119.025631. Saur D, et al (2003), "Sensitivity and Interrater Agreement of CT and Diffusion-Weighted MR Imaging in Hyperacute Stroke," AJNR 24, 878-885, https://www.ncbi.nlm.nih.gov/pubmed?linkname=pubmed pubmed\&from uid=12748088.

Saver JL, et al. (1999), "Infarct Volume as a Surrogate or Auxiliary Outcome Measure in Ischemic Stroke Clinical Trials," Stroke 30, 293-298, https://doi.org/10.1161/01.str.30.2.293.

Saver JL (2007), "Hemorrhage After Thrombolytic Therapy for Stroke: The Clinically Relevant Number Needed to Harm," Stroke 38, 2279-2283, https://doi.org/10.1161/STROKEAHA.107.487009.

Saver JL, et al. (2015), “Stent-Retriever Thrombectomy after Intravenous t-PA vs. t-PA Alone in Stroke,” NEJM 372, 2285-2295, https://doi.org/10.1056/NEJMoa1415061.

Schaefer PW, et al. (2015), "Combining MRI with NIHSS Thresholds to Predict Outcome in Acute Ischemic Stroke: Value for Patient Selection," AJNR 36, 259-264, https://doi.org/10.3174/ajnr.A4103.

Scheinfeld MH, et al. (2017), "Imaging Scales and Techniques Used in the 2015 Endovascular Stroke Trials and AHA/ASA Revised Guidelines for Acute Intervention, Radiographics 37, 1605-1606, https://doi.org/10.1148/rg.2017160214, with online presentation: "A Radiologist's Guide to the Techniques and Scales Used in the 2015 Endovascular Stroke Trials and the Updated AHA/ASA Guidelines for Endovascular Stroke Treatment."

Schellinger PD, et al. (2003), "Imaging-Based Decision Making in Thrombolytic Therapy for Ischemic Stroke: Present Status," Stroke 34, 575-583, https://doi.org/DOI:10.1161/01.STR.0000051504.10095.9C.

Schellinger PD, Demaerschalk BM (2018), "Endovascular Stroke Therapy in the Late Time Window," Stroke 49, 2559-2561, https://doi.org/10.1161/STROKEAHA.118.021003.

Schriger DL, et al. (1998), "Cranial Computed Tomography Interpretation in Acute Stroke: Physician Accuracy in Determining Eligibility for Thrombotic Therapy," JAMA 279,1293-1297, https://doi.org/10.1001/jama.279.16.1293.

Schröder J, et al. (2014), "Validity of Acute Stroke Lesion Volume Estimation by Diffusion-Weighted Imaging-Alberta Stroke Program Early Computed Tomographic Score Depends on Lesion Location in 496 Patients With Middle Cerebral Artery Stroke," Stroke 45, 3583-3588, https://doi.org/10.1161/STROKEAHA.114.006694.

Schröder J, Thomalla G (2017), "A Critical Review of Alberta Stroke Program Early CT Score for Evaluation of Acute Stroke Imaging," Frontiers in Neurology 7, article 245, https://doi.org/10.3389/fneur.2016.00245.

Schröder J, et al. (2018), "Impact of Lesion Load Thresholds on Alberta Stroke Program Early Computed Tomographic Score in Diffusion-Weighted Imaging," Frontiers in Neurology 9, article 273, https://doi.org/10.3389/fneur.2018.00273. Schwamm LH, et al. (2018), "Intravenous Thrombolysis in Unwitnessed Stroke Onset: MR WITNESS Trial Results," Annals of Neurology 83, 980-993, https://doi.org/10.1002/ana.25235.

Scott WA (1955), "Reliability of Content Analysis: The Case of Nominal Scale Coding," Public Opinion Q 19, 321-325, https://doi.org/10.1086/266577.

Seker F, et al. (2016), "Comparison of Four Different Collateral Scores in Acute Ischemic Stroke by CT Angiography," Journal of Neurointerventional Surgery 8, 1116-1118, https://doi.org/10.1136/neurintsurg-2015-012101.

Selim M, et al. (2002), "Predictors of Hemorrhagic Transformation After Intravenous Recombinant Tissue Plasminogen Activator: Prognostic Value of the Initial Apparent Diffusion Coefficient and Diffusion-Weighted Lesion Volume," Stroke 33, 2047-2052, https://doi.org/10.1161/01.str.0000023577.65990.4e.

Sené M, et al. (2017), "Metrology is Key to Reproducing Results," Nature 547, 397-399, https://doi.org/10.1038/547397a. Senn S (2003), "Disappointing Dichotomies," Pharmaceutical Statistics 2, 239-240, https://doi.org/10.1002/pst.90.

Senn S (2013), "Being Efficient About Efficacy Estimation," Statistics in Biopharmaceutical Research 5, 204-211, https://doi.org/10.1080/19466315.2012.754726.

Senn S (2018), “Statistical Pitfalls of Personalized Medicine," Nature 563, 619-621, https://doi.org/10.1038/d41586-018-07535-2. Seyedsaadat SM, et al. (2020), "Location-Specific ASPECTS Paradigm in Acute Ischemic Stroke: A Systematic Review and Meta-Analysis," AJNR 41, 2020-2026+Supp., https://doi.org/10.3174/ajnr.A6847.

Seyedsaadat SM, et al. (2021), "Differential Contribution of ASPECTS Regions to Clinical Outcome after Thrombectomy for Acute Ischemic Stroke," AJNR 41, 1104-8; https://doi.org/10.3174/ajnr.A7096.

Shankar GS, et al. (2018), "Correlation of Human Height with Foot Length in Indian Individuals," International Journal of Anatomy and Research 6, 4943-4946, https://doi.org/10.16965/ijar.2017.516.

Shanks DR, et al. (2002), "A Re-examination of Probability Matching and Rational Choice," Journal of Behavioral Decision Making 15, 233-250, https://doi.org/10.1002/bdm.413. 
Shannon CE (1948), “A Mathematical Theory of Communication,” The Bell System Technical Journal 27, 379-423, 623-656; reset, with corrections and new pagination at http://math.harvard.edu/ ctm/home/text/others/shannon/entropy/entropy.pdf. Sheth KN, et al. (2010), "Comparison of Hematoma Shape and Volume Estimates in Warfarin Versus Non-WarfarinRelated Intracerebral Hemorrhage," Neurocritical Care 12, 30-34, https://doi.org/10.1007/s12028-009-9296-7.

Sheth SA, et al. (2018), "Regional Contributions to Poststroke Disability in Endovascular Therapy," Interventional Neurology 7, 533-543, https://doi.org/10.1159/000492400.

Sheth SA, et al. (2019), "Machine Learning-Enabled Automated Determination of Acute Ischemic Core From Computed Tomography Angiography," Stroke 50, 3093-3100, http://doi.org/10.1161/STROKEAHA.119.026189.

Siegel S (1956), Nonparametric Statistics: For the Behavioral Sciences, New York: McGraw-Hill.

Siegel S, Castellan NJ (1988), Nonparametric Statistics for the Behavioral Sciences, $2^{\text {nd }}$ ed., New York: McGraw-Hill. Sillanpää N, et al. (2011), "CT Perfusion ASPECTS in the Evaluation of Acute Ischemic Stroke: Thrombolytic Therapy Perspective," Cerebrovascular Diseases Extra 1, 6-16, https://doi.org/10.1159/000324324.

Sillanpää N, et al. (2012), "The Clot Burden Score, the Boston Acute Stroke Imaging Scale, the Cerebral Blood Volume ASPECTS, and Two Novel Imaging Parameters in the Prediction of Clinical Outcome of Ischemic Stroke Patients Receiving Intravenous Thrombolytic Therapy," Neuroradiology 54, 663-672, https://doi.org/10.1007/s00234-011-0954-z. Silver B, et al. (2001), "Improved Outcomes in Stroke Thrombolysis with Pre-specified Imaging Criteria," Canadian Journal of the Neurological Sciences 28, 113-119, https://doi.org/10.1017/s031716710005277x.

Sim J, Wright CC (2005), "The Kappa Statistic in Reliability Studies: Use, Interpretation, and Sample Size Requirements," Physical Therapy 85, 257-268, https://doi.org/10.1093/ptj/85.3.257.

Simon HA (1956), "Rational Choice and the Structure of the Environment," Psychological Review 63, 129-138.

Simon HA (1997), Administrative Behavior, $4^{\text {th }}$ ed., New York: The Free Press.

Sims JR, et al. (2009), "ABC/2 for Rapid Clinical Estimate of Infarct, Perfusion, and Mismatch Volumes," Neurology 72, 2104-2110, https://doi.org/10.1212/WNL.0b013e3181aa5329.

Singer OC, et al. (2008), "Risk for Symptomatic Intracerebral Hemorrhage after Thrombolysis Assessed by DiffusionWeighted Magnetic Resonance Imaging," Annals of Neurology 63, 52-60, https://doi.org/10.1002/ana.21222.

Singer OC, et al. (2009), "Risk Assessment of Symptomatic Intracerebral Hemorrhage After Thrombolysis Using DWI-ASPECTS,” Stroke 40, 2743-2748, https://doi.org/10.1161/STROKEAHA.109.550111.

Smaldino P (2019), "Better Methods Can’t Make Up for Mediocre Theory,” Nature 575, 9-10, https://doi.org/10.1038/d41586-019-03350-5.

Smith, E. S. (1944), Automatic Control Engineering, New York: McGraw-Hill; available at Hathi Trust Digital Library, http://babel.hathitrust.org/cgi/pt?id=mdp.39015021260719; view=1up;seq=31.

Spearman C (1904), "The Proof and Measurement of Association Between Two Things," The American Journal of Psychology 15, 72-101; reprinted, ibid. 100 (No. 3/4, Special Centennial Issue, 1987), 441-471, https://doi.org/10.2307/1422689.

Spence I, Wainer H (2005), "William Playfair: A Daring Worthless Fellow," in Wainer H, Graphic Discovery: A Trout in the Milk and Other Visual Adventures, Princeton University Press, Chapter 3, pp. 24-27.

Spence I (2005), "No Humble Pie: The Origins and Usage of a Statistical Chart," Journal of Educational and Behavioral Statistics 30, 353-368, https://doi.org/10.3102/10769986030004353.

Spence I (2006), “William Playfair and the Psychology of Graphs," Joint Statistical Meetings 2006 Proceedings, American Statistical Association, Alexandria,VA, pp. 2426-2436, http://citeseerx.ist.psu.edu/viewdoc/summary?doi=10.1.1.488.6937. Spiotta AM, et al. (2015), "Impact of the ASPECT Scores and Distribution on Outcome among Patients Undergoing Thrombectomy for Acute Ischemic Stroke," Journal of Neurointerventional Surgery 7, 551-558, https://doi.org/10.1136/neurintsurg-2014-011195.

Sreenivasan S, et al. (2016), "Measuring Glioma Volumes: A Comparison of Linear Measurement Based Formulae with the Manual Image Segmentation Technique," Journal of Cancer Research and Therapeutics 12, 161-168, https://doi.org/10.4103/0973-1482.153999.

Srinivasan A, et al. (2006), "State-of-the-Art Imaging of Acute Stroke," Radiographics 26, S75-S95, https://doi.org/10.1148/rg.26si065501.

Steinbeck J (1954), Sweet Thursday, New York: Viking Press; reprinted, New York: Penguin, 1996 (the cited pagination). Stevens SS (1946), "On the Theory of Scales of Measurement," Science 103, 677-680,

https://doi.org/10.1126/science.103.2684.677.

Stevens SS (1958), “Measurement and Man," Science 127, 383-389, https://doi.org/10.1126/science.127.3295.383.

Stigler SM (1986), The History of Statistics, Cambridge MA: Harvard University Press.

Straus MA (2007), "Processes Explaining the Concealment and Distortion of Evidence on Gender Symmetry in Partner Violence," European Journal on Criminal Policy and Research 13, 227-232, https://doi.org/10.1007/s10610-007-9060-5. Streib CD, et al. (2019), "Infarct Volume Predicts Hospitalization Costs in Anterior Circulation Large-Vessel Occlusion Stroke," AJNR 40, 51-58, https://doi.org/10.3174/ajnr.A5917.

Strogatz S (2019), "The Math Equation That Tried to Stump the Internet," The New York Times, https://www.nytimes.com/2019/08/02/science/math-equation-pedmas-bemdas-bedmas.html. 
Page 96 ASPECTS, The Mismeasure of Stroke: A Metrological Investigation

Students of the University of Michigan (1877), "Confusion Worse Confounded," The Chronicle IX:1 (Oct. 13), 8-9; available via books.google.com.

Sullivan DC, et al. (2015), "Metrology Standards for Quantitative Imaging Biomarkers," Radiology 277, 813-825, https://doi.org/10.1148/radiol.2015142202.

Sullivan RL (1968), “The People v. Malcolm Ricardo Collins,” 68 Cal. 2d 319 (Supreme Court of California).

Sun Y (2017), "Coding of Data," in Allen M, ed., The SAGE Encyclopedia of Communication Research Methods, Thousand Oaks CA: SAGE Publications, vol. 1, pp. 148-151.

Sundaram VK, et al. (2019), "Automated ASPECTS in Acute Ischemic Stroke: A Comparative Analysis with CT Perfusion," AJNR 40, 2033-2038, https://doi.org/10.3174/ajnr.A6303.

Suss RA, Pinho MC (2020), “ASPECTS Distorts Infarct Volume Measurement” (Letter), AJNR 41, E28 (published online April 2), https://doi.org/10.3174/ajnr.A6485.

Tan BYQ, et al. (2017), "Validation of Serial Alberta Stroke Program Early CT Score (ASPECTS) as Outcome Predictors for Intravenous Thrombolysis in Acute Ischemic Stroke," Journal of the Neurological Sciences 381, Supplement S, 73-74, https://doi.org/https://doi.org/10.1016/j.jns.2017.08.259.

Tanne D, et al. (2002), "Markers of Increased Risk of Intracerebral Hemorrhage After Intravenous Recombinant Tissue Plasminogen Activator Therapy for Acute Ischemic Stroke in Clinical Practice: The Multicenter rt-PA Acute Stroke Survey," Circulation 105, 1679-1685, https://doi.org/10.1161/01.CIR.0000012747.53592.6A.

Thibault S, et al. (2021), "Tool Use and Language Share Syntactic Processes and Neural Patterns in the Basal Ganglia," Science 374, $841+1-14$ online, http://doi.org/10.1126/science.abe0874.

Thijs VN, et al. (2000), "Is Early Ischemic Lesion Volume on Diffusion-Weighted Imaging an Independent Predictor of Stroke Outcome? A Multivariable Analysis," Stroke 31, 2597-2602, https://doi.org/10.1161/01.str.31.11.2597.

Thomalla G, et al. (2018), "MRI-Guided Thrombolysis for Stroke with Unknown Time of Onset," NEJM 379, 611-622, https://doi.org/10.1056/NEJMoa1804355.

Thomas JB (1969), An Introduction to Statistical Communication Theory, New York: Wiley.

Thomson W (1862), "On the Age of the Sun's Heat," Macmillan's Magazine, No. 29, March, Cambridge: Macmillan and Co., pp. 388-393; available via books.google.com.

Thomson W (1883), "Electrical Units of Measurement. A Lecture delivered at the Institution of Civil Engineers on May 3 ...," in -, Popular Lectures and Addresses, Volume 1, Constitution of Matter, London: Macmillan, 1889, pp. 73-136; available via archive.org.

Timpone VM, et al. (2015), "Percentage Insula Ribbon Infarction of $>50 \%$ Identifies Patients Likely to Have Poor Clinical Outcome Despite Small DWI Infarct Volume," AJNR 36, 40-45, https://doi.org/10.3174/ainr.A4091.

Tirschwell DL, et al. (2002), "Shortening the NIH Stroke Scale for Use in the Prehospital Setting," Stroke 33, 2801-2806, https://doi.org/10.1161/01.str.0000044166.28481.bc.

Tomsick TA (1994), "Sensitivity and Prognostic Value of Early CT in Occlusion of the Middle Cerebral Artery Trunk," AJNR 15, 16-18; http://www.ajnr.org/content/ajnr/15/1/16.full.pdf.

Toni D, et al. (1995), "Progressing Neurological Deficit Secondary to Acute Ischemic Stroke," Arch Neurol 52, 670-675, https://doi.org/10.1001/archneur.1995.00540310040014.

Toni D, et al. (1996), "Hemorrhagic Transformation of Brain Infarct: Predictability in the First 5 Hours from Stroke Onset and Influence on Clinical Outcome," Neurology 46, 341-345, https://doi.org/10.1212/wnl.46.2.341.

Townsend RR (2018), "The Value in an Ambulatory Blood-Pressure Registry,” NEJM 378, 1555-1556,

https://doi.org/10.1056/NEJMe1802369.

Tribus M (1961), Thermostatics and Thermodynamics, Princeton: D. Van Nostrand.

Tropp HS (1984), "Origin of the Term Bit," Annals of the History of Computing 6, 152-155.

Turc G, et al. (2019), "European Stroke Organisation (ESO) - European Society for Minimally Invasive Neurological Therapy (ESMINT) Guidelines on Mechanical Thrombectomy in Acute Ischemic Stroke," Journal of Neurointerventional Surgery 11, 535-538 + full article online, 1-30, https://doi.org/10.1136/neurintsurg-2018-014569.

Uddin LQ, et al. (2017), "Structure and Function of the Human Insula," Journal of Clinical Neurophysiology 34, 300-306, https://doi.org/10.1097/WNP.0000000000000377.

Uno J, et al. (2018), "Mechanical Thrombectomy for Acute Anterior Cerebral Artery Occlusion," World Neurosurgery 120, e957-e961, https://doi.org/10.1016/i.wneu.2018.08.196.

Vagal A, et al. (2019), “Automated CT Perfusion Imaging for Acute Ischemic Stroke: Pearls and Pitfalls for Real-world Use," Neurology 93, 888-898, https://doi.org/10.1212/WNL.0000000000008481.

Vaidyanathan U, et al. (2009), "Startle Reflex Potentiation During Aversive Picture Viewing as an Indicator of Trait Fear," Psychophysiology 46, 75-85, https://doi.org/10.1111/j.1469-8986.2008.00751.x.

van der Worp HB, et al. (2001), "Reproducibility of Measurements of Cerebral Infarct Volume on CT Scans," Stroke 32, 424-430, https://doi.org/10.1161/01.str.32.2.424.

van der Zwan A, Hillen B (1991), "Review of the Variability of the Territories of the Major Cerebral Arteries," Stroke 22, 1078-1084, https://doi.org/10.1161/01.str.22.8.1078. 
van der Zwan A, et al. (1992), "Variability of the Territories of the Major Cerebral Arteries," Journal of Neurosurgery 77, 927-940, https://doi.org/10.3171/ins.1992.77.6.0927.

van der Zwan A, et al. (1993), "A Quantitative Investigation of the Variability of the Major Cerebral Arterial Territories," Stroke 24, 1951-1959, https://doi.org/10.1161/01.str.24.12.1951.

van Oest R (2019), “A New Coefficient of Interrater Agreement: The Challenge of Highly Unequal Category Proportions," Psychological Methods 24, 439-451; https://doi.org/10.1037/met0000183.

Vecchio, T. J. (1966), "Predictive Value of a Single Diagnostic Test in Unselected Populations," NEJM 274, 1171-1173, https://doi.org/10.1056/NEJM196605262742104.

Velleman PF, Wilkinson L (1993), "Nominal, Ordinal, Interval, and Ratio Typologies are Misleading," The American Statistician 47, 65-72, https://doi.org/10.1080/00031305.1993.10475938.

Venema E, et al. (2017), "Selection of Patients for Intra-Arterial Treatment for Acute Ischaemic Stroke: Development and Validation of a Clinical Decision Tool in Two Randomised Trials," The BMJ 357,j1710, https://doi.org/10.1136/bmi.j1710. Verhulst P-F (1845), "Recherches mathématiques sur la Loi d'Accroissement de la Population," Nouveaux Mémoires de l'Académie Royale des Sciences et Belles-Lettres de Bruxelles 8, 1-38 + figure,

http://www.med.mcgill.ca/epidemiology/Hanley/anniversaries/ByTopic/Verhulst1844.pdf

Villablanca JR (2010), “Why Do We Have a Caudate Nucleus?” Acta Neurobiologiae Experimentalis 70, 95-105, https://www.ncbi.nlm.nih.gov/pubmed?linkname=pubmed_pubmed\&from_uid=20407491.

Vogt G, et al. (2012), "Initial Lesion Volume Is an Independent Predictor of Clinical Stroke Outcome at Day 90: An Analysis of the Virtual International Stroke Trials Archive (VISTA) Database," Stroke 43, 1266-1272,

https://doi.org/10.1161/STROKEAHA.111.646570.

Voleti S, et al. (2021), "Correlation of Alberta Stroke Program Early Computed Tomography Score with Computed Tomography Perfusion Core in Large Vessel Occlusion in Delayed Time Windows," Stroke 52, 498-504;

https://doi.org/10.1161/STROKEAHA.120.030353.

Voltaire [Arouet F-M] (1772), La Bégueule, Conte Moral, Lausanne: François Grasset; available via books.google.com. von Kummer R, et al. (1994), "Sensitivity and Prognostic Value of Early CT in Occlusion of the Middle Cerebral Artery Trunk," AJNR 15, 9-15, https://www.ncbi.nlm.nih.gov/pubmed?linkname=pubmed pubmed\&from uid=8141071. von Kummer R, et al. (1996), "Interobserver Agreement in Assessing Early CT Signs of Middle Cerebral Artery Infarction," AJNR 17, 1743-1748, https://www.ncbi.nlm.nih.gov/pubmed?linkname=pubmed_pubmed\&from uid=8896631. von Kummer R, et al. (1997), “Acute Stroke: Usefulness of Early CT Findings before Thrombolytic Therapy,” Radiology 205, 327-33, https://doi.org/10.1148/radiology.205.2.9356611.

Waddington MM (1974), Atlas of Cerebral Angiography with Anatomic Correlation, Boston: Little, Brown.

Wald A (1949), "Statistical Decision Functions," The Annals of Mathematical Statistics 20, 165-205.

Wald A (1950a), "Basic Ideas of a General Theory of Statistical Decision Rules," Proceedings of the International Congress of Mathematics 1, Cambridge, 231-243.

Wald A (1950b), Statistical Decision Functions, New York: Wiley.

Waldron A, et al. (2011), Physical Review Style and Notation Guide, Ridge NY: The American Physical Society, https://cdn.journals.aps.org/files/styleguide-pr.pdf.

Wang GK, et al. (2012), "Measuring the Volume of the Lentiform Nucleus in Healthy Chinese Adults Based on High-resolution MRI," Chinese Journal of Medical Imaging Technology 28(1), 15-18; detailed abstract at ResearchGate, https://www.researchgate.net/publication/282751414 Measuring the volume of the lentiform nucleus in healthy Chinese adults based on high-resolution MRI. Wardlaw JM, Sellar R (1994), “A Simple Practical Classification of Cerebral Infarcts on CT and Its Interobserver Reliability," $A J N R$ 15, 1933-1939, https://www.ncbi.nlm.nih.gov/pubmed?linkname=pubmed pubmed\&from uid=7863945.

Wardlaw JM, et al.(1999a), “Thrombolysis for Acute Ischaemic Stroke (Review)," Cochrane Database of Systematic Reviews 1999, Issue 4 (full authorship: Wardlaw JM, del Zoppo G, Yamaguchi T). This version is superseded in the available Cochrane Database by Wardlaw et al. 2014/2016, but it was kindly provided by the lead author.

Wardlaw JM, et al. (1999b), "Can Stroke Physicians and Neuroradiologists Identify Signs of Early Cerebral Infarction on CT?” JNNP 67, 631-653, https://doi.org/10.1136/jnnp.67.5.651.

Wardlaw JM, et al. (2007), "Factors Influencing the Detection of Early CT Signs of Cerebral Ischemia: An InternetBased, International Multiobserver Study," Stroke 38, 1250-1256, https://doi.org/10.1161/01.STR.0000259715.53166.25. Wardlaw JM, et al. (2010), "A Large Web-Based Observer Reliability Study of Early Ischaemic Signs on Computed Tomography. The Acute Cerebral CT Evaluation of Stroke Study (ACCESS)," PLoS ONE 5(12), e15757, https://doi.org/10.1371/journal.pone.0015757.

Wardlaw JM, et al. (2014), "Imaging Perfusion Deficits, Arterial Patency and Thrombolysis Safety and Efficacy in Acute Ischaemic Stroke. An Observational Study of the Effect of Advanced Imaging Methods in the Third International Stroke Trial (IST-3), a Randomised Controlled Trial,' Efficacy and Mechanism Evaluation 1:1, https://doi.org/10.3310/eme01010. Wardlaw JM, et al. (2014/2016), “Thrombolysis for Acute Ischaemic Stroke (Review)," Cochrane Database of Systematic Reviews 2014, Issue 7, Art. CD000213 (full authorship: Wardlaw JM, Murray V, Berge E, del Zoppo G); with 2015 Feedback, The Cochrane Collaboration / John Wiley \& Sons Ltd, 2016, https://doi.org/DOI:10.1002/14651858.CD000213.pub3. 
Page 98 ASPECTS, The Mismeasure of Stroke: A Metrological Investigation

Wardlaw JM, et al. (2015), "Association between Brain Imaging Signs, Early and Late Outcomes, and Response to Intravenous Alteplase after Acute Ischaemic Stroke in the Third International Stroke Trial (IST3): Secondary Analysis of a Randomised Controlled Trial," Lancet Neurology 14, 485-496, https://doi.org/10.1016/S1474-4422(15)00012-5. Webb AJS, et al. (2015), "Accuracy of the ABC/2 Score for Intracerebral Hemorrhage Systematic Review and Analysis of MISTIE, CLEAR-IVH, and CLEAR III," Stroke 46, 2470-2476, https://doi.org/10.1161/STROKEAHA.114.007343. Weik MH (1996), Communications Standard Dictionary, $3^{\text {rd }}$ ed., New York: Chapman \& Hall.

Weintraub MI (2006), "Thrombolysis (Tissue Plasminogen Activator) in Stroke A Medicolegal Quagmire," Stroke 37, 1917-1922, https://doi.org/10.1161/01.STR.0000226651.04862.da.

Weir B (1987), Aneurysms Affecting the Nervous System, Baltimore: Williams \& Wilkins.

Weir NU, et al. (2006), "How Well Does ASPECTS Predict the Outcome of Acute Stroke Treated with IV tPA?" Stroke 36, 2110-2115, https://doi.org/10.1212/01.wnl.0000228221.44334.73.

Whiteley WN, et al. (2014), "Targeting Recombinant Tissue-Type Plasminogen Activator in Acute Ischemic Stroke Based on Risk of Intracranial Hemorrhage or Poor Functional Outcome: An Analysis of the Third International Stroke Trial," Stroke 45, 1000-1006, https://doi.org/10.1161/STROKEAHA.113.004362.

Wilson AT, et al. (2018), "Minds Treating Brains: Understanding the Interpretation of Non-contrast CT ASPECTS in Acute Ischemic Stroke," Expert Review of Cardiovascular Therapy 16, 143-153, https://doi.org/10.1080/14779072.2018.1421069. Wollenweber FA, et al. (2019), "Functional Outcome Following Stroke Thrombectomy in Clinical Practice," Stroke 50, 2500-2506, https://doi.org/10.1161/STROKEAHA.119.026005.

Won SY, etal. (2018), "Excellent Accuracy of ABC/2 Volume Formula Compared to Computer-assisted Volumetric Analysis of Subdural Hematomas," PLoS ONE 13(6), e0199809, https://doi.org/10.1371/journal.pone.0199809.

Woo D, et al. (1999), "Does the National Institutes of Health Stroke Scale Favor Left Hemisphere Strokes?" Stroke 30, 2355-2359, https://doi.org/10.1161/01.str.30.11.2355.

Wu O, et al. (2015), "Role of Acute Lesion Topography in Initial Ischemic Stroke Severity and Long-Term Functional Outcomes," Stroke 46, 2438-2444, https://doi.org/10.1161/STROKEAHA.115.009643.

Wusthoff CJ, et al. (2011), "Risk of Later Seizure After Perinatal Arterial Ischemic Stroke: A Prospective Cohort Study," Pediatrics 127, e1550-e1557, https://doi.org/10.1542/peds.2010-1577.

Yaghi S, et al. (2017), "Treatment and Outcome of Hemorrhagic Transformation After Intravenous Alteplase in Acute Ischemic Stroke: A Scientific Statement for Healthcare Professionals From the American Heart Association/American Stroke Association," Stroke 48, e343-e361, https://doi.org/10.1161/STR.0000000000000152.

Yan J, et al. (2013), "Comparison Between the Formula 1/2ABC and 2/3Sh in Intracerebral Parenchyma Hemorrhage," Neurological Research 35, 382-388, https://doi.org/10.1179/1743132812Y.0000000141.

Yang W, et al. (2013), "Volume Quantification of Acute Infratentorial Hemorrhage with Computed Tomography: Validation of the Formula 1/2ABC and 2/3SH," PLoS ONE 8(4), art. e62286, https://doi.org/10.1371/journal.pone.0062286. Yerushalmy J (1947), "Statistical Problems in Assessing Methods of Medical Diagnosis, with Special Reference to X-Ray Techniques," Public Health Reports 62, 1432-1449, https://doi.org/10.2307/4586294.

Yoo AJ, et al. (2009), "MRI-Based Selection for Intra-arterial Stroke Therapy: Value of Pretreatment Diffusion-Weighted Imaging Lesion Volume in Selecting Patients with Acute Stroke Who Will Benefit from Early Recanalization," Stroke 40, 2046-2054, https://doi.org/10.1161/STROKEAHA.108.541656.

Yoo AJ, et al. (2012), "Infarct Volume Is a Pivotal Biomarker After Intra-Arterial Stroke Therapy," Stroke 43, 1323-1330; https://doi.org/10.1161/STROKEAHA.111.639401.

Yoo AJ, et al. (2014), "Impact of Pretreatment Noncontrast CT Alberta Stroke Program Early CT Score on Clinical Outcome After Intra-Arterial Stroke Therapy," Stroke 45, 746-751, https://doi.org/10.1161/STROKEAHA.113.004260.

Yoo AJ, et al. (2016), "Effect of Baseline Alberta Stroke Program Early CT Score on Safety and Efficacy of Intra-arterial Treatment: A Subgroup Analysis of a Randomised Phase 3 Trial (MR CLEAN)," Lancet Neurology 15, 685-694, https://doi.org/10.1016/S1474-4422(16)00124-1.

Zadeh LA (1965), "Fuzzy Sets," Information and Control 8, 338-353, https://doi.org/10.1016/S0019-9958(65)90241-X. Zellner A (1986), "Bayesian Estimation and Prediction Using Asymmetric Loss Functions," Journal of the American Statistical Association 81, 446-451, https://doi.org/10.2307/2289234.

Zhao K-J, et al. (2009), "A Precise, Simple, Convenient and New Method for Estimation of Intracranial Hematoma Volume - the Formula 2/3Sh,” Neurological Research 31, 1031-1036, https://doi.org/10.1179/174313209X385662.

Zhao K-J, et al. (2010), "Comparisons of 2/3Sh Estimation Technique to Computer-assisted Planimetric Analysis in Epidural, Subdural and Intracerebral Hematomas," Neurological Research 32, 910-917, https://doi.org/10.1179/016164110X12681290831441.

Zivin JA, et al. (1985), “Tissue Plasminogen Activator Reduces Neurological Damage After Cerebral Embolism," Science 230, 1289-1292, https://doi.org/10.1126/science.3934754.

Zwick R (1988), “Another Look at Interrater Agreement,” Psychological Bulletin 103, 374-378,

https://doi.org/10.1037/0033-2909.103.3.374. 


\section{Special Symbols and Abbreviations}

$\S(\S \S)$ : section(s) or subsection(s).

II : pilcrow, indicating a paragraph break (inside a quote).

$/ /:$ an ASPECTS threshold, e.g., 6//5, cutting between 6 and 5 .

$\equiv:$ inherently equal to, defined as (used in mathematical expressions).

$\approx$ : approximately equal to; may read "approximately" or "about" in text.

$<, \leq ; \geq$, > : less than, less than or equal to; greater than, greater than or equal to.

$\ll ; \gg$ : much less than; much greater than.

$\subset:$ is a proper superset of (or a kind or component of), e.g., CBF $\subset$ CTP.

$\bar{x}$ : arithmetic mean of the values of $x$.

$|x|$ : absolute value of $x$, undirected difference from 0 .

$\wedge$ : to the exponent of, e.g., $x^{\wedge} 0.5$ means the square root of $x$.

$\sum p_{i}$ : sum (add up) $p_{1}+p_{2}+\ldots+p_{N}(i=$ index, 1 to $N)$.

$\mathbf{2 S h / 3}$ : ellipsoid volume measure using area $(S) \times$ height $(h)$.

$\boldsymbol{A B C / 2}$ : ellipsoid volume measure using perpendicular diameters.

ACA: anterior cerebral artery (or its territory).

ACCESS: Acute Cerebral CT Evaluation of Stroke Study.

AChA: anterior choroidal artery (or its territory).

ADC: apparent diffusion coefficient from DWI (acute infarct causes dark signal).

ALIC: anterior limb of internal capsule.

ASPECTS: Alberta Stroke Program Early CT Score, scoring 10 (no acute infarct in MCA territory) to 0.

ASTER: Contact Aspiration vs. Stent Retriever for Successful Revascularization.

CBF or CBV: Cerebral blood flow or volume by CTP.

cm: centimeters; $\mathbf{c m}^{2}$ : square centimeters; $\mathbf{c m}^{3}$ : cubic centimeters $(\mathrm{cc}, \mathrm{ml}, \mathrm{mL})$.

CT: x-ray computed tomography, generally plain.

CTP: CT perfusion.

DAWN: Revascularization with Solitaire FR Device Versus Best Medical Therapy in the Treatment of Acute Stroke Due to Anterior Circulation Large Vessel Occlusion Presenting Within Eight Hours of Symptom Onset.

DEFUSE 3: Endovascular Therapy Following Imaging Evaluation for Ischemic Stroke 3.

DWI: diffusion-weighted imaging in MRI (acute infarct causes bright signal).

ECASS [I] II III: European Cooperative Acute Stroke Study.

EIC: early ischemic change(s).

ESCAPE: Randomized Assessment of Rapid Endovascular Treatment of Ischemic Stroke.

ESMINT: European Society for Minimally Invasive Neurological Therapy.

ESO: European Stroke Organisation.

EXTEND-IA: Extending the Time for Thrombolysis in Emergency Neurological Deficits-Intra-Arterial.

$\boldsymbol{H}$ : Shannon's entropy of a probability distribution.

$\boldsymbol{H}_{\text {Max }}$ : maximum entropy for a given $N,=\log _{2} N$.

$\boldsymbol{H}_{\text {Rel }}$ : relative entropy $=H / H_{\max }($ for $H$ 's given $N)$.

HERMES: Highly Effective Reperfusion Evaluated in Multiple Endovascular Stroke Trials.

HI: hemorrhagic infarction (distinguished from frank parenchymal hematoma, $\mathrm{PH}$ ). 
Page 100 ASPECTS, The Mismeasure of Stroke: A Metrological Investigation

$i$ : item number in a list of items denoted by a variable (e.g., probabilities $p_{i}$ ).

IC: internal capsule.

IMS III: Interventional Management of Stroke III Trial.

IST-3 (or IST): Third International Stroke Trial.

IV: intravenous.

$\boldsymbol{\kappa}$ : kappa statistic, a coefficient of agreement.

$\log _{2}$ : base-2 logarithm.

LVO: large vessel occlusion.

M1-M6: the ASPECTS convexity regions.

MCA: middle cerebral artery (or its territory).

ml, mL: milliliters, $\mathrm{cm}^{3}$

MR CLEAN: Multicenter Randomized Clinical Trial of Endovascular Treatment for Acute Ischemic Stroke

MRI: magnetic resonance imaging.

in the Netherlands.

MR RESCUE: Mechanical Retrieval and Recanalization of Stroke Clots Using Embolectomy.

mRS: modified Rankin Scale, scoring 0 (no deficit) to 6 (dead).

MT: mechanical thrombectomy.

$\boldsymbol{n}$ : any numerical variable, e.g., the number of items in a set or sample.

$N$ : number of different items or different outcomes (with their probabilities $p_{i}$ ) in a probability distribution.

NIHSS: National Institutes of Health Stroke Scale, scoring 0 (normal) to 42.

NINDS: National Institute of Neurological Disorders and Stroke.

NPV, PPV: negative and positive predictive values.

$\boldsymbol{p}$ : a probability, e.g., of getting $\geq$ the observed difference if the null hypothesis is true.

$\boldsymbol{p}_{i}$ : a probability from a complete probability distribution, $i=1, \ldots, N$ (order is arbitrary).

PCA: posterior cerebral artery (or its territory).

PH: parenchymal hematoma (distinguished from petechial or diffuse microhemorrhage, HI)

PISTE: Pragmatic Ischaemic Stroke Thrombectomy Evaluation.

PLIC: posterior limb of internal capsule.

PWI: perfusion-weighted imaging in MRI.

Q.E.D.: quod erat demonstrandum, that which was to be proved.

RCT: randomized clinical trial.

REVASCAT: Randomized Trial of Revascularization with Solitaire FR Device Versus Best Medical Therapy in the Treatment of Acute Stroke Due to Anterior Circulation Large Vessel Occlusion Presenting Within $\boldsymbol{r}_{\mathrm{s}}$ : Spearman rank-order correlation coefficient.

Eight Hours of Symptom Onset.

rsNR: reference standard neuroradiologist (for the ACCESS database).

SD: standard deviation.

sICH: symptomatic intracerebral hemorrhage.

STICH [I] II: Surgical Trial in Lobar Intracerebral Haemorrhage.

SWIFT PRIME: Solitaire With the Intention For Thrombectomy as Primary Endovascular Treatment.

THERAPY: Assess the Penumbra System in the Treatment of Acute Stroke.

THRACE: Thrombectomie des Artères Cerebrales.

TJC: The Joint Commission (formerly JCAHO).

tPA: recombinant tissue plasminogen activator (sometimes quoted as rt-PA), alteplase, Activase ${ }^{\circledR}$.

WM: cerebral white matter. 